


\section{Enst Whit Lony}

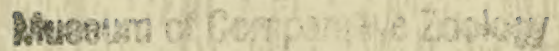

Harved Univoraby

HARVARD UNIVERSITY
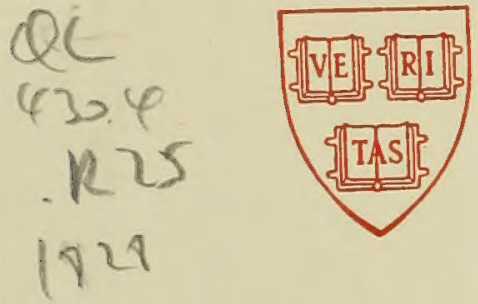

LIBRARY

OF THE

DEPARTMENT OF MOLLUSKS

IN THE

MUSEUM OF COMPARATIVE ZOOLOGY

Gift of:

RICHARD 1. JOHNSON 


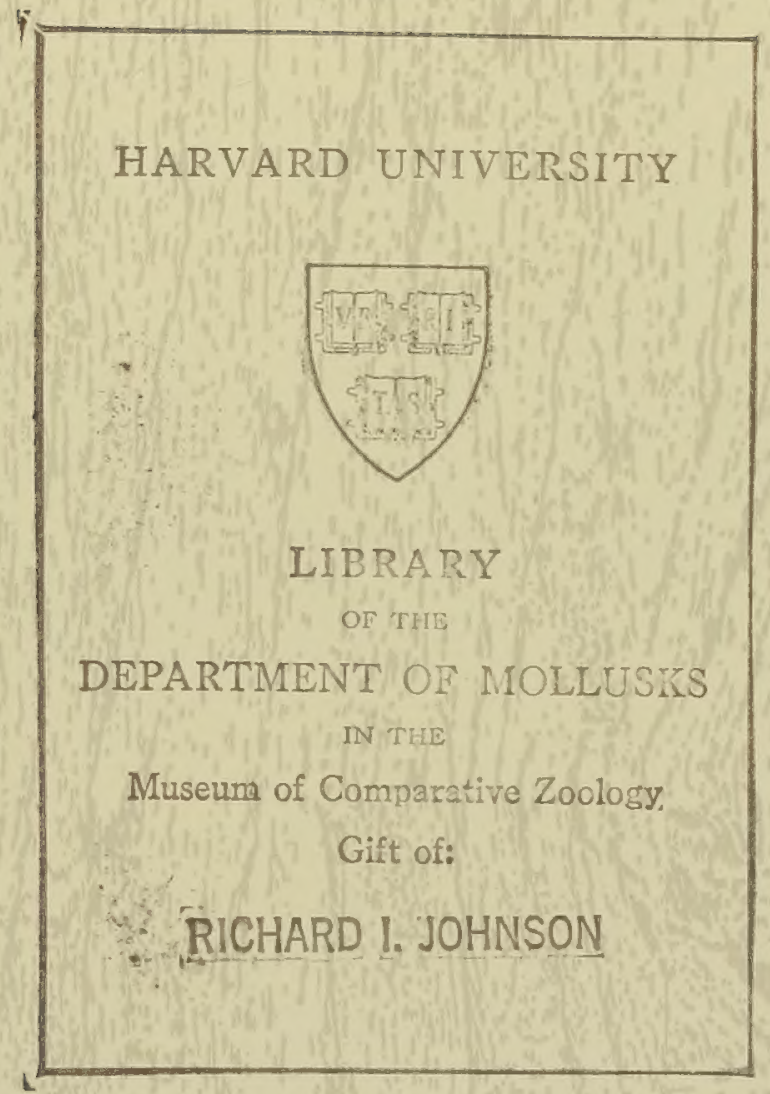







\title{
HISTOIRE NATURELLE,
}

\section{GENERALE ET PARTICULIÈRE}

\section{DES MOLLUSQUES,}

TANT DES ESPĖCES QUE L'ON TROUVE AUJOURD'HUI VIVANTES, QUE DES DÉPOUILLES FOSSILES DE CELLES QUI N'EXISTENT PLUS;

\section{CLASSÉS}

D'APRÈS LES CARACTÈRes ESSENTIRLS QUE PRÉSENTENT CES ANIMAUX ET LEURS COQUULLES.

DÉDIÉ E A S. A. R.

\section{$M^{\text {GR }}$ LE DUC D'ANGOULEME;}

\author{
Par M. le Baron DE fÉrussaC,
}

\begin{abstract}
Directeun de la Sociétí du Bulletin universez pour za Propagation des connotssances scientifieues et industrigles, Officter supérieur au Corps rotal d'État-major, Chef de Diviston au Ministère du Commerce et des Manufactungs, Chevalize de Saint-Lodis et de la Légron d'Honneur;

Membre de la Commission centrale de la Sociétẻ de Géographie, de la Société Royale des Ántiquaires de France, des Sociẻtés d'Bncouragement, Philomatique, d'Horticulture et d'Histoire naturelle de Paris; des Sociétes Linnéennes de Caen et de Bordeaux (section de Paris), d'Agriculture, Sciences et Arts de Châlons et d'Agen, de l'Académie Royale de La Rochelle, etc.; des Académies des Curieux de la nature de Bonn, Royale des Sciences médicales de Madrid et des Sciences naturelles de Philadelphie; des Sociétés d'Histoire naturelle de Moscou, Halle, Marbourg, Francfort, Hannau et de l'Helvétie; des Sociétés géologique, médico-botanique et horticulturale de Londres, minéralogique de Jéna, botanique de Ratisbonne, physico-médicale du Bas-Rhin ; physiographique de Lund, d'Agriculture d'Ettlingen, de Cassel, du royaume de Bavière, économique de Potsdam, patriotique de la Silésie, Royale des Beaux-Arts et de Littérature de Gand, du Lycée de New-Yorck, et de la Réunion polytechnique de Bavièré, etc.
\end{abstract}

\section{PARIS,}

CHEZ ARTHUS BERTRAND, LIBRAIRE, RUE HAUTEFEUILLE, $\mathbb{N}^{\circ} 23$. 
(-) 


\section{HISTOIRE NATURELLE}

\section{DES APLYSIENS.}

PREMIER E FAMILL

DE L'ORDRE

DES TECTIBRANCHES.

PAR M. SANDER RANG,

OFficier aU CORPS roYal de la marine, Membre associé de L'áadémie royale de ta rochelle,

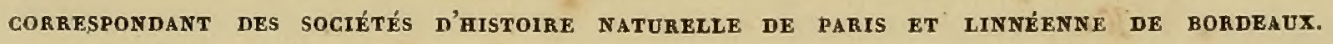

\section{PARIS.}

DE L'IMPRIMERIE DE FIRMIN DIDOT, IMPRIMEUR DU ROI, DE L'INSTITUT ET DE LA MARINE,

RUE JACOB, No 24 .

MDCCCXXVIII, 
$2 n+12 x+342=$ 


\section{INTRODUCTION.}

LA Monographie que je soumets au jugement des naturalistes, a particulièrement pour but la connoissance de l'un des genres les plus riches et les plus intéressants dè la classe des Mollusques. On sera peut-être surpris que j'aie entrepris un semblable travail, après les beaux mémoires de Bohadsch et de MM. Guvier et delle Chiaje sur le même sujet; mais on concevra que j'ai pu le faire, quand on connoîtra le nombre considérable d'espèces dont j'augmente le genre Aplysie. D'un autre côté, je ferai observer que ces savants ont eu un but tout différent de celui que je me propose; ils ont cherché à faire connoître aussi complètement que possible l'anatomie de l'Aplysie, et ils ne se sont occupés que secondairement de la distinction de quelques espèces, tandis qu'au contraire prenant leurs observations pour base de mon travail, j'établis, de la manière qui m'a paru la plus convenable, les caractères du genre, et je m'attache surtout à en décrire toutes les espèces, dont plus de la moitié étoit encore inédite. J'ai eu également pour but de faire connoître, touchant les mœurs, les habitudes et les propriétés de ces animaux, tout ce que j'ai eu occasion d'observer par moi - même pendant le cours de plusieurs voyages lointains. Enfin, pour rendre cet ouvrage complet et réellement utile, j’ai dû ajouter à mes propres observations ce qui a été dit sur les Aplysies par les auteurs anciens et par les modernes, afin que les naturalistes trouvassent réuni tout ce que l'on sait sur les Mollusques qui font le sujet de ce travail.

Je n'ai cependant pas la prétention de croire que cette Monographie soit complète; je n'ai même pas l'espoir qu'elle soit exempte d'erreurs, et je ne la regarde, pour ainsi dire, que comme un travail préparatoire à celui bien plus étendu que je prévois déja que l'on pourra faire un jour sur le même sujet; du moins j'en juge par l'intérêt qu'inspirent les Aplysiens aux naturalistes qui explorent des régions nouvelles, et par l'étonnante promptitude avec laquelle se sont trouvés réunis dans un court espace de temps les matériaux qui m'ont servi, tant ceux que j'ai recueillis moi-même que ceux qui m’ont été généreusement communiqués par plusieurs naturalistes.

Quinze à vingt espèces nouvelles sont le fruit de mes recherches pendant le cours d'un voyage de deux années. Ces espèces, observées vivantes, étudiées avec 
soin aussi long-temps qu'il m'étoit possible de les conserver dans cet état, et dessinées avec une scrupuleuse attention pour les caractères, formaient, lorsque je revins en France, une Monographie assez considérable, surtout en y réunissant les espèces déja décrites.

M. Cuvier, qui malgré le nombre et l'importance des travaux dont il est sans cesse occupé, aime encore à reporter ses regards sur cette belle classe des Mollusques, dont le premier il a dévoilé les lois d'organisation, a daigné encourager l'essai que je me proposois de faire, en ajoutant à mes matériaux ceux qu'il avoit en sa possession dans la belle collection du Cabinet d'anatomie. Cette généreuse communication; faitè avec tout le désintéressement qui caractérise le célèbre fondateur de ce précieux établissement, a singulièrement contribué à enrichir mon travail. J'ai trouvé au Jardin du Roi un grand nombre d'individus dont une partie m'étoit entièrement inconnue, et qui avoient été recueillis soit par Péron dans les mers aus-, trales, soit par MM. Mathieu à l'Ile-de-France, Richard à Cayenne, Cuvier, Risso, Polidor Roux sur les bords de la Méditerranée, d'Orbigny à la Rochelle, Quoy et Gaimard pendant leur premier voyage autour du monde, et Plée aux Antilles, où la mort l'a si cruellement frappé au milieu de ses intéressantes recherches. D'autres espèces, plus récemment recueillies, venoient encore d'y être déposées, et m'offroient un intérêt d'autant plus grand que mon ami M. Lesson, à qui on les doit, m'avoit déja remis de jolis dessins et de précieuses notes prises sur ces mêmes animaux, lorsqu'il les observoit vivants.

J'ai donc pu étudier avec loisir cette belle suite d'espèces; et, selon le desir du savant à qui j'en suis redevable, j’ai nommé celles qui n'étoient pas encore décrites, et affectant à chacune un numéro correspondant à ma Monographie, je les ai distribuées selon l'ordre méthodique que j'avois déja adopté pour faciliter les déterminations spécifiques. De cette manière il sera facile de jugèr de l'exactitude que j’ai apportée dans cette partie de mon ouvrage; et si par la suite quelqu'un cherche à le rendre plus complet, on sera plus à même d'apprécier le choix de ma méthode, et d'éviter l'erreur des doubles emplois en examinant attentivement mes espèces et les comparant à mes descriptions.

D'autres naturalistes ont bien voulu contribuer encore à enrichir mon travail en mettant à ma disposition les matériaux qu'ils possédoient. M. de Férussac m'a fourni une nouvelle espèce dont je suis heureux de pouvoir lui faire hommage; je lui dois encore les dessins de deux autres espèces provenant du voyage à Java de Van-Hasselt. MI. Brogniart a également eu la bonté de me laisser visiter celles qu'il conserve dans l'esprit-de-vin, et qui ont été recueillies sur les côtes de France par MM. Audouin et Adolphe Brogniart: cette communication m'a été d'un grand secours, en ce qu'une partie de ces mèmes espèces avoit déja servi à M. de Blainville 
pour la Monographie qu'il a publiée en ı 822 . Enfin M. Lesson, si riche en découvertes de tout genre, n'a pas craint de priver le bel ouvrage à la rédaction duquel il travaille en ce moment, de la nouveauté de quelques-uns de ces Mollusques, et m'a fait part, comme je l'ai déja dit, de tout ce qu'il possédoit en ce genre.

Mais après tout, à quoi m'eussent servi toutes ces communications, et même les découvertes qui m'appartiennent en propre, si je n'avais rencontré, dans la bienveillance et le zèle ardent qui anime M. le baron de Férussac pour toutes les entreprises scientifiques, un secours puissant pour la publication d'un travail qui devenoit tout-à-coup si important? Nullement habitué aux rédactions de ce genre, et ne cultivant l'histoire naturelle que pour occuper mes loisirs sur des rivages lointains et distraire parfois mes pensées du souvenir de la patrie, il m'eût été peut-être difficile de traiter ce sujet; mais ce savant a bien voulu m'aider des conseils de son expérience, m'offrant en même temps d'admettre ma Monographie dans le bel ouvrage sur les Mollusques en général qu'il publie en ce moment : je n'ai pas dû balancer à accepter des offres si généreuses, et dès-lors j’ai pu espérer quelque réussite.

Maintenant que ma tâche est achevée, je suis heureux de pouvoir saisir l'occasion de témoigner publiquement à ce savant les sentiments de reconnoissance et d'affection qui m'attachent inviolablement à lui. 



\section{PREMIÈRE FAMILLE.}

\section{$\underline{\underline{20}}$ \\ LES APLYSIENS.}

Synonymis. Laplysiens de Lamarck; Dicères de Férussac; Aplysiens, Aplysiacea, de Blainville; Tentaculés de Latreille.

Caractènes. Forme générale. Corps charnu, oblong-alongé ou arrondi, bombé en-dessus, plat et généralement élargi en-dessous, conjoint avec le pied et ne formant qu'un tout avec lui, jamais divisé et ne se renfermant point dans une coquille; tểte, distincte; bouche, fendue en long; courerture, mantean étendu sur tout le corps, muni sur sa partie supérieure d'une fente longitudinale formant quelquefois, par la dilatation de ses bords, deux lobes latéraux plus ou moins distincts, propres à la natation, et qui se croisent sur la cavité branchiale; cavité branchiale, située à la partie moyenne et supérieure du corps, protégée, le plus souvent, par une membrane operculaire renfermant quelquefois une lame testacée et toujours par les bords du manteau; branclies, en forme de panache flottant dans cette cavité et quelquefois même en dehors; tentacules, au nombre de quatre; yeux, situés à la base et en avant des tentacules postérieurs; anus, placé en arrière des branchies; organes de la génération, séparés sur le même individu, mais liés par un canal extérieur; la vulve en avant des branchies, la verge près du tentacule droit antérieur.

Herbivores et carnassiers.

\section{HISTOIRE DE LA FAMILLE DES APLYSIENS.}

Les mollusques qui font le sujet de ce travail composent dans l'ordre des Tectibranches une petite famille extrêmement naturelle dans laquelle on ne compte encore que les genres Aplysie, Bursatelle et Actéon. Le premier de ces genres; qui est le plus étendu et le mieux connu, en est le type, et par conséquent celui sur qui reposent presque en entier les caractères des aplysiens.

M. de Lamarck, dans la première édition des Animaux sans vertèbres, réunissoit les Aplysies et les Dolabelles aux Bulléens, aux Téthys et aux Limaces, pour en faire sa famille des Limaciers. Quelque temps après, dans l'Ex 'xtrait de son cours, il en retira les Téthys et les Limaces, et substitua à la dénomination de Limaciers celle de Laplysiens, faisant la faute d'y ajouter en même temps le genre Sigaret, qui appartient évidemment aux Pectinibranches; enfin, dans la seconde édition de son bel ouvrage, il conserva les laplysiens, mais cette famille ne renferma plus que les Aplysies et les Dolabelles.

M. de Férussac, dans ses tableaux systématiques de la classification des mollusques, a 
adopté ce que M. de Lamarck avoit fait en dernier lieu;' mais il a ajouté aux deux genres que nous venons de citer, le Notarche de M. Cuvier, et l'Actéon d'Ocken.

M. de Blainville a de même admis la famille des aplysiens, ainsi que les genres qui venoient d'y être introduits par M. de Férussac; car le genre Elysie de MI. Risso n'est autre chose que l'Actéon d'Ocken, et il y a associé le genre Bursatelle, formé par M. de Blainville pour un mollusque extrêmement voisin des Aplysies.

Les Bursatelles et les Actéons, encore peu connus, et qui ne reposent jusqu'à présent que sur les deux espèces quii en sont les types, n'occuperont qu'une petite place dans notre travail, et c'est particulièrement le genre Aplysie, l'un des plus beaux de la classe des gastéropodes, et qui compose presque à lui seul cette petite famille, qui va nous occuper. C'est donc aussi à son histoire que nous devons nous reporter pour faire l'historique des aplysiens.

Quelques-uns des mollusques qui composent la famille des aplysiens étoient connus des anciens sous le nom de Lievre-marin, et, selon toutes les apparences, la connoissance de ces animaux remonte à l'époque la plus reculée; car les propriétés nombreuses et singulières que les premiers auteurs grecs et latins leur attribuent, portent à croire que le vulgaire les avoit distingués long-temps avant eux. Parmi les écrivains dont les livres sont venus jusqu’à nous, Nicandre, Dioscoride et Pline sont les premiers qui ont fait mention du lièvre-marin. Ces auteurs en ont traité en médecins plutôt qu'en naturalistes; aussi les nombreuses fables qu'ils débitent à son sujet, prouvent-elles combien la connoissance des productions de la nature est futile, lorsqu'elle n'est pas fondée sur une étude particulière de leur organisation et un examen scrupuleux de leurs propriétés. On est surpris, en lisant toutes les choses merveilleuses qu'ils rapportent sur ces mollusques, de n'y trouver que de courtes et mauvaises descriptions, et rarement même un caractère qui soit juste. Dioscoride compare le lièvre-marin à un petit Calmar, sans doute à cause de l'ouverture de son dos, et surtout de la liqueur qu'il répand. Pline en parle comme d'une masse informe qui n'auroit de ressemblance avec le lièvre de terre que par la couleur; cependant il est probable que le nom de lièvre-marin provient surtout d'un autre caractère de ressemblance qui semble se montrer dans la forme de la tête et dans celle des tentacules que l'on peut comparer à de longues oreilles. C'est pour cela sans doute qu'aujourd'hui encore les pêcheurs des côtes de La Rochelle le désignent sous le nom de chat de mer.

Pline parle de deux sortes de lièvre-marin; l'un qui habite la Méditerranée, et l'autre la mer des Indes : il ajoute que ce dernier ressemble au lièvre de terre par sa taille et ses poils qui sont seulement plus rudes.

On doit sans doute attribuer le peu de soin que les auteurs anciens ont mis à décrire ce mollusque à la répugnance que ne pouvoit manquer d'inspirer un animal d'un aspect aussi repoussant, et que d'ailleurs on s'accordoit à croire très-redoutable. La prévention des anciens contre le lièvre de mer étoit telle, qu'elle faisoit naittre un danger réel pour ceux qui auroient été tentés de l'observer de près. Apulée nous en laisse un exemple remarquable. Il venoit de contracter avec une riche veuve, nommée Pudentilla, un mariage qui Iui attira l'inimitié des parents de cette Romaine. Ceux-ci, fondant de grandes espérances sur son état et sa fortune, et ne désespérant pas entièrement des droits d'héritage dont cette union devoit les frustrer, essayèrent de perdre Apulée en l'accusant de magie et d'empoisonnement devant le proconsul Claudius Maximus, rapportant comme preuve 
principale, qu'il avoit engagé des pêcheurs, moyennant une forte somme d'argent, à lui apporter un lièvre-marin. Apulée plaida lui-même sa cause, et ne parvint qu'avec beaucoup de peine, mais surtout par le secours de son éloquence, à confondre ses ennemis, et à se faire renvoyer d'une accusation si grave.

On ne peut douter que l'intention d'Apulée ne fût d'observer ce mollusque, et de s'assurer s'il méritoit tout le mal qu'on en disoit; ce qu'il y a de certain, c'est qu'il est le premier qui l'ait bien examiné, aussi nous fournit-il à son sujet un trait caractéristique bien précieux. "Il a, dit-il, une singulière propriété, et qui a été ignorée jusqu'ici; c'est qu'étant d'ailleurs " dépourvu d'os, il en a cependant douze petits dans son ventre, et qui sont semblables "à des osselets ou astragales de cochon, réunis ensemble."

Æ̇lien a également parlé du lièvre-marin. "Il ressemble, dit-il, à un limaçon dont on “auroit enlevé la coquille.» Cette définition n'est pas sans importance; elle porte un caractère qui indique des rapports dont les auteurs modernes, comme nous le verrons plus loin, se sont sans doute servis pour reconnoître à quel genre de mollusque se rapportoit le lièvre-marin des anciens.

Rondelet, un des plus grands naturalistes dont s'honore la France, eu égard au temps où il vivoit, est le premier qui ait reconnu dans un mollusque des côtes du Languedoc le lièvre-marin des anciens. Il montre que c'est à tort que quelques écrivains ont cru voir cet animal dans différents poissons de la Méditerranée, puisque le mollusque dont il s'agit étoit très-venimeux, et même mortel pour ceux qui en auroient mangé, tandis que les poissons auxquels on le rapporte servent tous les jours de nourriture à l'homme. Cette remarque est judicieuse; car sans admettre que le lièvre-marin soit un poison mortel, on ne peut cependant rejeter tonte idée d'un principe malfaisant dans sa substance; opinion à laquelle la crainte, la crédulité et le temps auront donné plus de force, et qui bientôt aura été accompagnée de circonstances plus ou moins merveilleuses.

L'animal observé par Rondelet étoit bien fait sans doute pour rappeler le lièvre-marin des anciens, tant par son aspect hideux que par l'humeur qui découle de son corps et l'odeur vireuse et nauséabonde qu'il répand autour de lui. Rondelet, qui l'avoit observé dans l'état de vie, a dû ètre frappé des rapports que ses caractères offroient avec ceux que les anciens nous ont transmis sur leur lièvre-marin. Son corps charnu, épais, mollasse, et les contractions violentes qui, quelquefois, lui donnent un aspect singulier, lui expliquèrent pourquoi Pline le compare à une masse informe. La nature de sa substance et la liqueur épaisse et abondante qu'il verse autour de lui, lui rappelèrent que Dioscoride le compare à un Calmar, et lorsque l'animal, venant à ramper, prit toute son extension, il dut certainement reconnoître le caractère que nous devons à Æ̇lien lorsqu'il dit que le lièvre-marin ressemble à un limaçon privé de sa coquille; enfin, comme nous venons de le remarquer, il dut voir dans l'aspect hideux et la puanteur de ce mollusque l'origine des fables qui ont été débitées à son sujet. Il y a lieu de croire que ce naturaliste ne fut pas aussi satisfait lorsqu'il compara la couleur de son mollusque à celle du lièvre-marin telle que Pline la rapporte, car il prend un détour pour démontrer qu'elle est bien la même. En effet, les anciens, dit-il, nommoient couleur de lièvre, la couleur d'une sorte d'olive noirâtre; or la couleur de cette olive peut bien être celle du lièvre-marin de Rondelet; mais rien ne prouve que ce soit celle du mollusque des anciens, car il n'est pas permis de douter que Pline n'ait entendu par la couleur du lièvre de terre celle du poil de ce qua- 
drupède. "Dans notre mer, dit l'auteur latin, cet animal est une masse informe qui ne " ressemble au lièvre que par sa couleur; mais dans la mer des Indes, il lui ressemble "aussi par sa grosseur, et par son poil qui est seulement plus rude (I).»

D'après cela nous sommes très-portés à croire que le mollusque de Rondelet est bien du même genre que le lièvre-marin des anciens, mais que ce n'est pas la même espèce, comme nous essayerons de le démontrer plus loin. Quoi qu'il en soit, Rondelet n'en a pas moins le mérite de la découverte; c'est bien certainement à lui que nous devons la connoissance de l'animal dont les anciens ont tant parlé, et cela d'une manière si extraordinaire.

Un fait bien singulier, c'est que Rondelet, qui paroît s'être attaché à recueillir tous les moyens de prouver l'analogie de son animal avec celui de Pline, a cependant négligé une preuve tout-à-fait convaincante, et dont Apulée lui fournissoit l'occasion en observant que le lièvre-marin a dáns le ventre douze petits osselets. Ce caractère, que nous avons déja signalé comme étant très-important dans une semblable discussion, ne doit plus nous laisser de doute sur l'heureux rapprochement dû à Rondelet. C'est Bohadsch qui le premier l'a reconnu sur l'animal qui a servi à ses recherches anatomiques, et depuis il a été signalé par M. Cuvier comme appartenant à toutes les espèces d'Aplysies.

Nous remarquerons aussi qu'Apulée, qui paroît avoir si bien examiné le lièvre-marin, puisqu'il a porté ses recherches jusque dans l'organisation interne, n'a point parlé du rudiment de coquille qui protége les branchies et le cour dans l'espèce qui étoit à sa disposition; il n'est cependant pas probable qu'il ait échappé à son investigation; il y a plutôt lieu de croire que le trouvant si mince, si flexible et peut-être tout membraneux, il n'y ait point fixé son attention d'une manière particulière.

Cette coquille n'a point échappé à Rondelet, et il falloit que ce grand naturaliste fût bien frappé de l'identité de son animal avec celui des anciens pour que la considération de cette pièce essentielle, dont personne n'avoit parlé avant lui, ne l'ébranlât pas dans l'idée de sa découverte du lièvre-marin.

Ce que nous devons à ce naturaliste au sujet de l'animal qui nous occupe ne se borne pas là; il est le premier qui en ait donné une figure accompagnée d'une assez bonne description, et qui ait reconnu les lobes latéraux pour de véritables nageoires.

Malheureusement, après avoir si bien fait connoître le lièvre-marin, il semble en oublièr les caractères pour y joindre un mollusque qui en diffère évidemment, et qui appartient au genre Téthys.

Rondelet donne encore une seconde figure de lièvre-marin, mais inexacte et accompagnée d'une description insuffisante. Cette figure, qui n'indique aucune cavité branchiale, a beaucoup embarrassé les auteurs qui depuis ont traité le genre Aplysie. Quant à nous, nous la jugeons indéterminable.

Nous ne sommes pas encore aussi éclairés sur le lièvre-marin de la mer des Indes que sur celui de la Méditerranée, et maintenant que la découverte de Rondelet nous apprend à quel genre de mollusque répond le dernier, nous pouvons, en quelque sorte, nous assurer si le premier mérite également d'en être rapproché. Les mêmes auteurs anciens en ont parlé, mais moins en détail. Elien nous fournit cependant les caractères suivants,

(1) Pline, lib. X, cap. 48. 
qui doivent décider la question : “Il est, dit-il, hérissé de poils très-durs, et capables de " blesser; il nage toujours dans la partie supérieure des eaux, et ne se tient point au fond; “ il se meut avec vitesse. Il nous semble que nous ne devons pas voir dans cette définition un mollusque du genre du lièvre-marin, mais bien des caractères propres à quelque espèce de poisson; sans doute les auteurs à qui nous devons la connoissance de cet animal ne l'avoient pas vu par eux-mêmes, et peut-être qu'ils n'avoient établi ce rapprochement, que sur le récit des propriétés fabuleuses dont le prétendu lièvre-marin des Indes a été le sujet tout aussi bien que celui de la Méditerranée. Nous n'insisterons pas davantage sur ce point, qui semble placé hors de notre sujet, et qui se montre enveloppé d'un voile obscur que nous tenterions vainement de soulever. En conséquence, nous ne reviendrons plus sur le lièvre-marin des Indes.

Le premier auteur qui ait copié Rondelet, est Gesner ( I); il se contenta de lui emprunter sa description et ses figures sans rien ajouter à ce qu’il en avoit dit : il est seulement bon de remarquer qu'il ne rangea plus le lièvre-marin parmi les poissons, comme l'avoit fait Rondelet, mais qu'il le comprit dans sa division des animaux mous, empruntée à Aristote.

Aldrovande (2) a fait comme Gesner; il s'est borné à répéter ce que ses prédécesseurs avoient écrit, et à reproduire les figures des trois espèces de Rondelet.

Fabius Columna (3) a donné une excellente description de la troisième espèce, que nous avons dit être un e Tethys. Ce naturaliste auroit donc pu la séparer des deux premières; non-seulement il ne l'a pas fait, mais il a ajouté au genre une quatrième espèce, qui est une Doris, et qui par conséquént ne se rapproche pas davantage du lièvre-marin que de la Téthys.

Linnée n'avoit point vu, à ce qu'il paroît, de lièvre-marin; et ce qu'il savoit de ce mollusque, il le devoit, de mềme que Fabius Columna, Aldrovande et Gesner, à Rondelet. Aussi ne doit-on pas être surpris de voir que, très-embarrassé des espèces citées, il les ait placées dans le genre Lernée, qui lui-même renfermoit déja plusieurs animaux très-différents entre eux. Dans ses quatrième et sixième éditions les lièvres-marins furent compris sous cette dénomination générique.

Bohadsch (4) publia, quelque temps après, son beau travail sur le lièvre-marin qu'il eut l'occasion d'observer sur les côtes d'Italie. Ce savant anatomiste n'examine point si le mollusque dont il s'agit est bien le lièvre-marin des anciens, et s'il doit entrer ou non dans le genre Lernée; il ne s'occupe qu'à en décrire l'organisation, et à en rechercher les propriétés particulières. Ce trạvail, vu le temps où il a été exécuté, est un des plus beaux qui ait été fait sur ce genre de mollusque. Voici de quelle manière M. Cuvier en parle: "Son travail, dit-il, auroit presque rendu le mien inutile, si ses procédés anatomiques " avoient été assez délicats, surtout s'il avoit eu, losqu'il se livra à ces recherches, des "connoissances comparées de l'organisation des genres voisins. "

Les recherches de Bohadsch furent faites sur l'espèce même que, d'un avis général, on croit être celle des anciens, et qui, comme nous le verrons plus tard, présente en effet,

(I) Gesner, Animalium mar, ordo. XIII de Mollibus, p. 193.

(2) Aldrovande, de An. Exs., p. So.

(3) Fabius Goluma, de Aquatil. et T'errest. obs., p. xuviij.

(4) BoHadsGr, de quibustam animalibus marinis. 
plus que toutes les autres, des caractères propres à autoriser ce rapprochement. Ce naturaliste entra dans quelques détails sur les mours, l'accouplement et les propriétés de l'humeur âcre que ces mollusques sécrétent. Se fondant sur son expérience, il paroît disposé à croire une partie des récits de Dioscoride et de Pline sur leurs propriétés malfaisantes. Il adopta le nom de Lernée que Linnée leur avoit donné, on ne sait trop poüquoi. Mais dans le même moment à peu près, Linnée lui-même venoit de faire paroître la dixième édition du Systema Naturae, où le lièvre-marin ne se trouve plus confondu avec les Lernées, mais constitue un genre à part pour les trois lièvres-marins de Rondelet, sous le nom de Téthys. Bohadsch convient qu’il auroit encore été à temps de changer le nom de Lernée en celui de Téthys, ajoutant qu'il ne crut pas devoir le faire, ayant reconnu que les deux noms ne valoient pas mieux l'un que l'autre. «Il est bien aise, dit-il encore, de procurer "par là à M. Linnée l'occasion, qu'il aime tant, de faire un genre nouveau.»

En effet celui-ci ne la manqua pas; éclairé par le beau travail anatomique que le premier venoit de publier, il fit une grande réforme dans cette partie de sa douzième édition. Avec son genre Téthys il en fit deux, l'un sous le nom de Laplysie, Laplysia, se composa du premier et du second lièvre-marin de Rondelet, et l'autre, qui conserva le nom de Téthys, se trouva formé du troisième. Ces deux genres établis sur les bonnes descriptions de Bohadsch, se sont ainsi maintenus. Gmelin (1) les adopta, et ajouta au genre Laplysie l'A. fasciata, nouvelle espèce qui venoit d'être décrite dans le voyage en Barbarie de M. Poiret. Il est probable que c'est de cette même espèce que parle Bohadsch, lorsqu'il dit : “ Il y " en a qui sont plus grandes (que l'A plysie dépilante), et dont la couleur est d'un pourpre " éclatant. » Gmelin crut devoir faire une correction au nom générique imposé par Linnée, et en effet ce naturaliste, comme l'a observé depuis M. Cuvier, s'étoit sans doute trompé en écrivant Laplysie; il avoit probablement voulu mettre Aplysie, nom que les anciens donnoient à une sorte d'éponge, et qui signifie ce qui ne peut être lavé. Nous ne parlerons donc plus désormais du lièvre-marin que sous le nom d'Aplysie, qui a été généralement adopté par les savants qui, depuis, ont parlé de cet intéressant mollusque.

Nous devons encore à Linnée le nom spécifique du lièvre-marin que l'on croit être celui des anciens; voulant lui consacrer une dénomination qui rappelât l'une des propriétés qu'ils lui attribuoient, il le nomma $A$. depilans. Malheureusement il n'avoit pu, préalablement, se convaincre si cet animal possédoit réellement la propriété de dépiler. Nous connoissons donc actuellement deux espèces d'Aplysies, les $A$. depilans et fasciata, bien caractérisées; plus les figures des deux premiers lièvres-marins de Rondelet, dont l'une, sans doute, se rapporte à l'une de ces espèces.

Si par les derniers travaux dont nous venons de rendre compte, la connoissance des aplysies fit des progrès sensibles, il est vrai de dire aussi qu'ensuite, elle demeura assez longtemps, à peu près au point où les travaux de Bohadsch et de Linnée l'avoient conduite. En effet, Barbut (2), dans son Genera vermium, et Bruguière (3), dans l'Encyclopédie méthodique, n'ont fait que copier Bohadsch. MI. Bosc (4), qui se faisoit, comme ces deux

\footnotetext{
(1) GMelis, Syst. nat., p. 3103.

(2) Javus Barbut, the Generu vermium, p. 31, pl. 1II, f. 5 et 6 .

(3) Bruguiène, Encyclopédie méthodique, pl. 83 et 84 .

(4) Bosc, Ilist. nnt. des Vers, t. I, p. 7o, pl. 2.
} 
naturalistes, un scrupule d'adopter le changement apporté par Gmelin au nom de Laplysie, consacré par Iinnée, ne fit de plus qu'eux qu'ajouter une nouvelle espèce qu'il venoit de découvrir dans la baie de Charleston, sur les còtes de l'Amérique du nord. Du reste, les trois auteurs que nous venons de citer adoptent, comme leurs prédécesseurs, úne partie des propriétés merveilleuses que les anciens attribuoient au lièvre-marin. Enfin, à l'époque où écrivoit M. Bosc, les naturalistes avoient encore si peu étudié ce genrè dé mollusque, ou plutôt ils connoissoient si peu nos rivages, que l'on ne croyoit pas que les Aplysies habitassent ailleurs que dans la Méditerranée.

La nouvelle espèce décrite par M. Bosc, sous le nom d' $A$. viridis, et qui n'a point été signalée depuis lui, est du reste très-incomplètement décrite et assez imparfaitement figurée; aussi a-t-elle toujours laissé beaucoup d'incertitude à son sujet.

M. Cuvier (I), danś son Tableau élémentaire de l'Histoire naturelle, publié en I $79^{8}$, ne donne point une attention spéciale aux Aplysies. Les mollusques, dans cet ouvrage, n'étoient encore distingués qu'en mollusques nus et en mollusques à coquille. Le genre Aplysie figure dans cette première division, ayant pour type l'Aplysie dépilante dont ce savant donne également une figure, empruntée à Bohadsch.

M. de Lamarck, peu de temps après, mit au jour sa première édition du Système des Animaux sans vertèbres, où ce genre figure sous le nom de Laplysie dans la division des mollusques céphalés, deuxième sous-division, les limaciers. Il ne fait également mention que de l'Aplysie dépilante, et ne nous apprend rien de plus à son sujet.

Le livre de M. Cuvier et celui de M. de Lamarck n'étoient que des tableaux systématiques de la classification des animaux; ils n'avoient donc pas pour but une connoissance approfondie des genres, mais bien celle des caractères qui pouvoient établir des rapprochements parmi eux, et contribuer à une classification naturelle. Ainsi donc nous ne devons pas être surpris si le genre Aplysie ne fut pas dès-lors traité par ces auteurs d'une manière plus spéciale. Ce ne pouvoit ètre qu'un retard de peu de durée; le genre Aplysie offroit trop d'intérêt pour l'étude des mollusques; d'ailleurs il restoit bien des lumières à acquérir à son sujet, et de fausses préventions à détruire; il appartenoit sans doute de remplir cette double tâche à celui qui, prêt à élever le bel édifice de son anatomie comparée, portoit ses regards sur toute la série des êtres, afin d'en coordonner les divisions et d'en dévoiler les lois respectives. M. Cuvier travailloit à sa grande division des mollusques; chaque fois que dans une classe il créoịt un ordre, ce n'étoit qu'après avoir fait une étude approfondie de l'organisation tant interne qu'externe des animaux qui le composoient et particulièrement de celui qui en formoit le type. Il dut en conséquence s'arrêter un moment sur le genre Aplysie, dont il formoit en effet le type de l'ordre des tectibránches de la classe des gastéropodes; c'est alors qu'il publia, dans les Annales du Muséum (2), un mémoire aussi plein d'intérêt que de savantes observations, et qui doit être regardé comme un modèle précieux dans ce genre de travail.

Dans ce mémoire, après avoir fixé notre attention sur l'Aplysie par le récit de son histoire et des fables auxquelles elle a donné lieu chez. les anciens, M. Cuvier en donne

(1) Cuvier, Tableau élémentaire de l'Histoire naturelle des animaux, p. 387 , pl. IX, f. 3.

(2) Annales du Muséum, t. II, p. 287, pl. 1-4. 
la description, bien plus détaillée et plus exacte qu'aucun auteur ne l'avoit encore fait; il signale six espèces d'Aplysie, l'A. depilans, l'A. fasciata et l'A.viridis, que nous connoissons déja; plus, trois autres espèces nouvelles, sous les noms d' $A$. punctata, Camelus et $a l b a$. Il ne cherche point à établir une distinction de groupes parmi ces six espèces; cependant il indique déja un caractère qui n'appartient qu'à certaines d'entre elles; c'est que les unes, telles que l' $A$. depilans et l' $A$. punclata, montrent une ouverture à la membrane de l'opercule, tandis que les autres en sont privées. Plus tard nous nous servirons nousmêmes de cette indication pour établir une division parmi les espèces de ce genre. Rendant hommage au beau travail de Bohadsch, comme nous l'avons dit plus haut, M. Cuvier reconnoît que ce naturaliste a fort bien décrit l'extérieur et la plupart des principaux viscères. Entrant ensuite en matière, il pénètre dans de plus grands détails que son prédécesseur, et semble ne laisser que peu de chose à ajouter.

A ce travail M. Cuvier a joint des planches dessinées par lui-même avec une exactitude admirable et un soin de détail presque minutieux. Les figures des espèces nouvelles qu'il signale y sont jointes, ainsi que le dessin de la coquille que porte l' $A$. fasciata, et qui n’avoit pas encore été représentée.

Les recherches et les observations de ce savant ne se sont pas bornées là; il a voulu s'assurer du cas que l'on doit faire de quelques-unes des fables débitées au sujet du lièvremarin, et en partie perpétuées par Bohadsch. Ayant observé plusieurs Aplysies vivantes, lors d'un voyage qu'il fit à Marseille, il se cónvainquit par sa propre expérience que ces animaux ne méritent pas la réputation qu'on leur a faite. Cette opinion, émise par une telle autorité, est bientôt devenue celle de presque tous les naturalistes qui ont écrit après lui.

On pense bien qu'après les beaux travaux de Bohadsch, et surtout de M. Cuvier, les naturalistes qui composèrent des ouvrages généraux, durent nécessairement emprunter à ces savants la plus grande partie de leurs documents. MMI. de Roissy, de Lamarck et de Férussac furent plus particulièrement dans ce cas; le premier, dans le Buffon de Sonnini; le second, dans une nouvelle édition des Animaux invertébrés, où il persiste toujours à garder le nom de Laplysie, et fait avec ce genre le type de la famille des laplysiens de la division des gastéropodes; et le troisième, dans le Dictionnaire classique d'Histoire naturelle. M. de Férussac admet les espèces de ses prédécesseurs, à l'exception de l'A. viridis de M. Bosc, qu'il introduit dans un nouveau genre de mollusque établi par Ocken, sous le nom d'Actéon, pour un petit animal des côtes du Devonshire. Outre cela, ce savant pense que le premièr lièvre-marin de Rondelet est l'espèce que Linnée a nommée depilans; mais il ne sait à quoi rapporter la seconde, qui, comme nous l'avons dit, offre incomplètement le caractère du genre dans lequel on l'a fait entrer.

Pour achever l'histoire du genre Aplysie, nous n'avons plus à examiner que les travaux de deux savants, dont les recherches, comme celles de Bohadsch et de MI. Cuvier, ont toujours pour base l'étude de l'organisation. L'un est un mémoire de M. delle Chiaje, professeur d'anatomie à Naples, et l'autre une monographie de M. de Blainville. Ces deux ouvrages, qui sont les derniers que l'on ait écrits sur les Aplysies, renferment plusieurs observations précieuses, et de bonnes descriptions d'espèces nouvelles ou peu connues.

M. delle Chiaje n'entre dans aucun détail sur l'histoire du genre; il ne cherche point 
non plus à prouver que l'aplysie est le lièvre marin des anciens; cependant il convient qu'il le croit, quoique ses observations se trouvent sur quelques points en opposition avec celles des anciens, car il a vainement cherché à reconnoître les propriétés qui ont été attribuées à ce mollusque. Le but de ce savant est de remplir quelques lacunes laissées par Bohadsch et M. Cuvier dans l'anatomie des aplysies, de faire connoître des espèces nouvelles, ainși qu'une méthode de les grouper, fondée sur l'observation de caractères importants.

Ses recherches anatomiques sont délicates et dirigées avec tout le talent qui étoit nécessaire pour glaner dans un champ si bien moissonné par deux habiles naturalistes. On pourroit presque dire de ce travail qu'il est le complément de celui de M. Cuvier.

En décrivant ses espèces, M. delle Chiaje rend un véritable service à la science, nonseulement parce qu'il en fait connoitre deux nouvelles, mais encore parce qu'il nous éclaire au sujet de celles déja connues : c'est ainsi qu'il nous donne des détails précieux sur l'A. Camelus de M. Cuvier, qui étoit si incomplétement décrite, et qu'il paroît avoir retrouvée. Il décrit et figure comme espèces nouvelles les $A$. neapolitana et Poliana, qui appartiennent aux mers de Naples. Ces descriptions ne laisseroient rien à désirer, si ce naturaliste avoit donné une plus grande attention aux caractères du test, et s'il l'avoit fait représenter à part. Il est également à regretter qu'il n'ait pas figuré l'A. Camelus comme il a figuré l'A. depilans. Quant à l'A. alba, Cuvier, il n'en tient aucun compte, jugeant que cette espèce n'est qu'un jeune individu de l'A. Camelus. Enfin il propose quelques changements dans la nomenclature. Convaincu par sa propre expérience que l' $A$. depilans ne possède pas la faculté de faire tomber le poil, que Bohadsch s'est accordé à lui reconnoître avec les anciens, il propose de changer śa dénomination, qui rappelle un fait inexact, en celle de leporina, qui auroit l'avantage de désigner l'espèce célèbre qui est le type du genre. Il propose encore de changer le nom de l'A. punctata en celui du naturaliste à qui on en doit la découverte : ce changement est fondé sur. ce que le caractère que porte cette dénomination n'est pas uniquement applicable à l'espèce à qui elle a été affectée. Nous ne blâmerons pas M. delle Chiaje d'avoir proposé ces changements, qui peuvent être', sous quelques rapports, considérés comme des rectifications réelles, et que d'ailleurs il est impossible de ne pas apprécier; cependant nous ne les adopterons pas, quand même il en seroit temps encore ( $r$ ), parce que nous savons combien on doit éviter d'encourager de semblables innovations. La perte d'espèces pour l'auteur qui les a découvertes et nommées est à nos yeux le moindre inconvénient; mais celui que l'on doit redouter, c'est la confusion qu'une semblable méthode ne tarderoit pas à jeter dans la synonymie en la compliquant, et l'embarras et les erreurs où cela pourroit conduire ceux qui n'en ont pas toujours sous les yeux une exacte.

La Monographie de M. delle Chiaje se compose donc de six espèces, plus l'A. viridis de M. Bosc, qu'il conserve au genre, mais dont il ne parle que dans une note, et pour la citer seulement. Ces six espèces sont l'A. fasciata, l' $A$. Camelus, l' $A$. neapolitana; l'A. depilans, l'A. punctata, et l'A. Poliana.

(1) Lorsque nous avons eu connoissance du travail de M. delle Chiaje, le nôtre et toutes nos planches étoient achevés. 
M. delle Chiaje a fait une découverte assez importante, mais qui malheureusement n'a servi qu'à l'égarer. Nous allons entrer dans quelques détails à ce sujet.

Les $A$. depilans, punctata, Poliana lui ayant offert un trou à la membrane supérieure de l'opercule, il lui vint dans l'idée que ce devoit être un caràctère générique, et que les A. fasciata, Camelus et neapolitana, qui n'en paroissoient pas munies, en avoient également un. Il se livra donc, pour parvenir à s'en assurer, à des recherches qui demandoient des moyens délicats. "Je dois convenir, dit-il avec modestie, que mes recherches ne " furent pas couronnées d'un heureux succès : j'introduisis à l'un des côtés de la cavité dans "laquelle est placée la coquille, le bec d'un très-petit tuyau de verre plein de mercure; "à peine le vif-argent eut-il rempli un angle de cette cavité, qu'aü moyen d'uné légère "pression de ses parois, il vint à jaillir du milieu de l'opercule, en me montrant un petit tube. »

Ce naturaliste a fait la mème découverte sur l'A. fasciata et l'A. neapolitana; il y a déja long-temps que nous l'avons aussi faite sur plusieurs espèces exotiques et sur l' $A$. fasciata; mais il l'a faite avant nous. Nous n'avons donc pas le mérite de la priorité, cependant nous avons celui d'avoir confirmé son observation sur un bien plus grand nombre d'espèces, et par conséquent de lui donner plus de poids. M. delle Chiaje, profitant de ce résultat, s'en sert pour l'établissement de deux sections; du moins il ne cite pas ce caractère dans la phrase indicative de ces sections, mais il en fait, dans chacune d'elles, le premier caractère de chaque espèce, ce qui revient à peu près au même; de manière que toutes les Aplysies, munies d'un tube à l'opercule, sont comprises dans la première section, tandis que celles qui n'ont qu'un trou simple composent la seconde.

Enhardi par sa découverte, ce savant s'est laissé entrainer à des rapprochements qu'il nous semble impossible d'admettre; il croit reconnoître que les $A$. depilans, punctata et Poliana, qui sont munies d'un trou à l'opercule, portent seules une coquille calcaire, tandis que les $A$. fasciata, Camelus et neapolitrna, qui montrent un tube, n'auroient qu'une coquille membraneuse. Il va plus loin, il rapporte aux premières la propriété de répandre une liqueur blanche et odorante, tandis que les secondes sécréteroient seules la liqueur pourprée dont elles se servent pour s'envelopper d'un nuage. Pour établir une distinction si importante, il falloit avoir sous les yeux un plus grand nombre d'espèces et d'individus de chacune d'elles à comparer; il falloit aussi en observer un plus grand nombre à l'état de vie.

Nous porterỏns une plus grande attention sur ce sujet, lorsque nous ferons connoître sur quelles bases nous avons établi notre classification des espèces.

Quoi qu'il en soit, nous pensons que la science doit à M. delle Chiaje des observations précieuses et qui contribuent puissamment à élever la connoissance du genre Aplysie au point où elle est en ce moment. Dans la même année où le mémoire de M. delle Chiaje a été publié en Italie, c'est-à-dire en $\mathbf{1}_{22}$, M. de Blainville (I) a donné dans le Journal de physique, et depuis, dans le Dictionnaire des sciences naturelles, une Monographic du genre Aplysie: ce savant n'avoit point eu connoissance de l'ouvrage de l'anatomiste de Naples, ainsi il n'a pu profiter de ses découvertes.

M. de Blainville est de tous les auteurs qui ont écrit sur les Aplysies, celui qui a donné

(1) Journal de Physique, $9^{6}$, janvier, 1823, p. 277, et Dictionnaire des Sciences naturelles, au mot Lière marin. 
le plus d'attention au rudiment de coquilles, aussi en a-t-il fait figurer quelques-uns; ce qui ajoute évidemment des caractères propres à la détermination des espèces qui les portent. C'est également à lui-que l'on doit la connoissance de l'A. limacina, qui n'en a point du tout.

La Monographie que nous examinons se compose de quatre espéces de plus que celle de M. delle Chiaje; et ce qu'il y a de remarquable, c'est que ces savants se sont à peu près rencontrés dans l'établissement de deux sections basées, en partie, sur la distinction des Aplysies malfaisantes de celles qui ne le sont pas. La première section de M. de Blainville se compose, en effet, des Aplysies orclinaires ou natatrices, et renferme les espèces qu'il regarde comme n'étant pas malfaisantes, tandis qu'il donne le nom d'Aplysies dépilantes à celles de sa seconde section qui renferme les Aplysies qu'il croit venimeuses. Ces deux sections répondent donc à celles de M. delle Chiaje. En effet nous remarquons que les $A$. depilans et punctata qui appartiennent à la seconde section de M. delle Chiaje font aussi partie de la seconde de M. de Blainville, tandis que, chez l'un comme chez l'autre de ces auteurs, $\mathrm{l}^{\prime} A$. fasciata se trouve dans la première. Il n'en est pas de même de l' $A$. alba, que l'un regarde comme étant la même que l' $A$. Camelus de sa première section. Elle se trouve dans la seconde de l'autre, tandis que l' $A$. Camelus, que M. de Blainville croit être la même que son $A$. vulgaris, se trouve sous ce dernier nom dans la première.

On se demánde ensuite pourquoi M. de Blainville fait entrer parmi les Aplysies venimeuses les $A$. unicolor, limacina et viridis, et dans les Aplysies ordinaires, les $A$. vulgaris, marginata et marmorata; examinons si les caractères de toutes ces espèces répondent comme elles le devroient aux caractères des sections qui les renferment.

Ce savant donne le nom de natatrices aux espèces de la première section; mais les $A$. depilans, punctata et viridis, qui sont de la seconde, ont les lobes latéraux suffisamment grands pour qu'on les croie susceptibles de s'en servir à la natation. L'A: depilans nage très-bien, nous avons eu lieu de nous en convaincre fréquemment sur les côtes de France.

Le corps des espèces de la première section est plus allongé,plus limaciforme; mais les $A$. depilans, limacina et viridis, sont toutes aussi allongées au moins que 1 'A. marginata et l' $A$. marmorala, qui sont de cette première section.

Le bouclier est muni en avant et en arrière d'une sorte d'oreille arrondie. Nous avouons que nous n'avons jamais observé sur les Aplysies l'oreille arrondie placée en avant de l'opercule que cite M. de Blainville; nous ne connoissons que celle qui est en arrière et que nous désignons sous le nom de siphon, parce qu'elle conduit l'eau aux branchies.

Les espèces de la première section n'ont rien de vireux, ni dans lodeur, etc.; mais l' $A$. fasciata qui est dans cette section a l'odeur de l' $A$. clepilans, pas aussi forte, il est vrai, mais assez cependant pour ne laisser aucune incertitude à ce sujet. Quant aux autres caractères, ils sont de peu de valeur, parce qu'ils ne reposent que sur des dimensions.

Si nous examinons ceux de la seconde section, nous voyons que la coquille dans les Aplysies qui la forment est plụs ou moins à découvert. Comment se fait-il donc qu'on y rencontre l' $A$. alba qui, au rapport de M. Cavier, à qui nous la devons, n'a point d'ouverture à la membrane supérieure de l'opercule? Pourquoi encore y voit-on figurer les $A$. limacina et viridis qui, d'après les descriptions qu'on en a données, n'ont point de coquille? 
M. de Blainville cite encore un caractère que nous n'avons jamais pu reconnoitre d'une manière satisfaisante, quoique nous l'ayons attentivement cherché sur un grand nombre d'espèces et d'individus. Il dit que les Aplysies qui composent la première section ont le côté gauche plus grand que le droit, tandis que celles qui forment la seconde ont au contraire le côté droit plus grand que le gauche. Nous avons quelquefois vu le contraire sur différents individus de la même espèce.

Nous ferons encore une observation, c'est que si l'on considère la figure et la description que ce savant donne de l'A. vulgaris, on ne peut s'empêcher d'y voir l'A. fasciata de M. Poiret; d'une autre part, en reportant la même figure dans son Traité de Malacologie, il la présente sous le nom d'A. depilans. Il y a nécessairement erreur quelque part. Il pense aussi que l'A. Camelus' est la même espèce que son $A$. vulgaris. S'il en étoit ainsi, nous ne voyons pas pourquoi on cesseroit d'adopter la première de ces dénominations qui a été donnée bien long-temps avant celle qu'il propose par le naturaliste à qui on en doit la découverte; du reste, les observations de M. delle Chiaje prouvent que l'A. Camelus diffère de l'A. fasciata, que nous croyons fermement être l' $A$. vulgaris de M. de Blainville.

Nous ajouterons encore que la figure 8 de la planche de ce naturaliste, qui représente, selon lui, l'A. depilans de la Mréditerranée, ne peut vraisemblablement pas répondre à cette espèce, parce qu'elle ne montre point d'ouverture à la membrane de l'opercule.

Ce savant ajoute peu de chose au sujet des propriétés attribuées aux Aplysies, et ne paroît disposé ni à les adopter ni à les repousser entièrement. Quant aux détails anatomiques, il les emprunte presque tous à Bohadsch et à M. Cuvier.

M. de Blainville, qui a soumis la grande division des Mollusques à un nouvel arrangement systématique, place le genre Aplysie dans l'ordre des monopleurobranches, section des hermaphrodites, classe des malacozoaires céphalophores.

Après ce travail on n'a rien publié de général sur le genre Aplysie: nous dirons seulement que M. Risso, dans son livre sur les Mollusques de l'Europe méridionale, a cherché à faire connoître quelques espèces qu'il donne comme nouvelles; mais leurs descriptions sont si incomplètes qu'il est impossible d'en tirer aucune lumière; que M. Savigny, dans le magnifique ouvrage sur l'Égypte, a figuré une espèce encore inconnue et d'un grand intérêt pour la science; que nous devons à M. Tilésius une espèce des mers de Chine, figurée dans le voyage de Krusenstern; et qu'enfin nos estimables amis MM. Quoy et Gaimard, Lesson et Garnot, zoologistes des deux dernières expéditions autour du monde, ont rapporté de précienx matériaux dont nous nous sommes emparé pour enrichir notre travail. Le concours de ces observations récentes, joint aux découvertes que nous avons été à même de faire dans nos voyages, nous ont mis dans le cas de publier une Monographie beaucoup plus complète, et qui, sans cela, n'offriroit sins doute que peu d'intérêt, après les savantes recherches des auteurs qui nous ont précédé dans cette matière.

La connoissançe des Dolabelles ne remonte pas, à beaucoup près, aussi haut que celle des Aplysies. Rumphius ( $\mathrm{I}$ ) est le premier qui en ait fait mention. On trouve une figure

(1) Rumpmus, Thesaurus cochlcarum, pl. X, f. 5, et pl. $\mathrm{XI}_{4}, \mathrm{f} . \mathrm{N}$. 
de l'animal qu'il nomme limax marina tertia, dans la planche.X de son ouvrage, et une figure de la coquille, qu'il appelle tertia species operculi callorum, dans sa planche XL. Mais une chose assez remarquable, c'est qu'il ne se doute pas que cette coquille appartient à l'animal qu'il a figuré, et ce n'est que long-temps après que ce fait a été reconnu.

Gmelin crut voir une Doris dans la figure du limax marina de Rumphius, et il en a fait la Doris verucosa. Barbut a suivi cet exemple et a reproduit cette figure sous le même nom et sous celui de citron de mer, et l'a coloriée à sa manière. Quant à la coquille, elle commençoit déja à se répandre dans les collections où elle étoit apportée des :mers de l'Inde. M. de Lamarck en fit un genre-dans sa première édition des Animaux sans vertèbres, sous le nom de Dolabelle, Dolabella, que tous les auteurs sè sont empressés d'adopter, et qui subsiste encore aujourd'hui. La forme de cette coquille a sans doute décidé ce naturaliste dans la dénomination qu'il lui a choisie. Ce fut quelque temps après que Péron rapporta deux individus de l'animal de la Dolabelle, et il conçut le premier l'heureuse idée que le limax marina tertia de Rumphius, étoit l'animal de l'operculum callorum. S'étant empressé de remettre ses deux Dolabelles à M. Cuvier, cet anatomiste en étudia l'organisation, et reconnut que dans leur intérieur elles ne différoient en rien des Aplysies, et que, si l'on pouvoit saisir quelque différence entre ces animaux, ce ne devoit être que dans la coquille, qui se montre toute calcaire chez la Dolabelle, et dans la disposition de son manteau qui porte des lobes serrés et nullement dilatés en nageoires comme dans les Aplysies, et qui montre un plan oblique postérieur. Ces différences lui paroissent de si peu d'importance que, dans son mémoire sur ce sujet, il ne craint pas de dire que "L'on pourroit, sans inconvénient, ranger la Dolabelle parmi les Aplysies, sous le nom d'A.'Dolabella." Cependant ce savant ne le fit pas, aimant mieux, pour le moment, laisser les choses comme M. de Lamarck les avoit établies. M. Cuvier joignit à son travail sur la Dolabelle une figure plus exacte de ce mollusque et de sa coquille. Il crut pouvoir affirmer que ces animaux répandent une liqueur pourprée, de même que les Aplysies, quoique Péron n'ait pu l'éclairer à ce sujet, parce qu'il retrouva dans l'opercule une substance glanduleuse, análogue à celle qui la sécrète chez ces dernières.

Conduit par l'analogie, le célèbre auteur des Animaux sans vertèbres a si bien conçu le mollusque auquel devoit appartenir cette coquille, qu’il l'a placé précisément comme il devoit l'être et de manière à ne point éprouver de changement, sous ce rapport, même après la connoissance acquise par M. Cuvier de son organisation générale.

M. de Lamarck; en établissant le genre Dolabelle, avoit assigné à la seule espèce qu'ịl connút le nom de $D$. callosa, à cause des callosités du sommet du test. M. Cuvier, à qui la science devoit d'avoir jeté des lumières sur ce genre, adoptant l'idée de Péron, changea cette dénomination en celle de $D$. Rumphii, et c'est sous ce nom qu'elle a généralement été citée depuis.

Ce genre, ainsi établi, s'est maintenu jusqu'à nous, sans éprouver de changements notables. Cependant M. Cuvier, dans son Règne animal (I), y rapporte trois espèces; l'une

(I) Cuvier, Règne animal, t. II, p. 398 
est celle dont nous venons de parler, et les deux autres sont désignées sous les noms de $D$. Rondeletii et de $D$. dolabrifera. Nous ignorons qui a établi la première, et sur quels caractères on s'est fondé pour la rapporter au genre Dolabelle; car nous ne voyons d'autre analogie entre la seconde espèce de lièvre marin de Rondelet et la Dolabelle, que celle qui existe entre elle et les Aplysies. D'ailleurs il n'y a point, dans la Méditerrannée, d'espèces de Dolabelles; ces mollusques sont propres à la mer des Indes et à celles de la Nouvelle-Hollande. Quạnt à la $D$. dolabrifera, elle a été établie par M. Cuvier, pour une Aplysie, rapportée de l'île de France par M. Mathicu. Elle se rapproche davantage des Dolabelles que celle de Rondelet; cependant elle n'a point de plan oblique, et l'on peut voir dans la série de nos espèces, où elle forme le type d'une section de même que la $D$. Rumphii, qu'elle diffère autant des Dolabelles de Lamarck que des Aplysies de Linnée; du reste ces différences de caractère peuvent tout au plus servir à établir des coupes dans un genre.

Bientôt M. de Lamarck, éclairé par les savantes recherches de M. Cuvier, sur le genre qu'il avoit créé, a donné, dans la seconde édition des Animanx sans vertèbres (I), un nouveau travail sur les Dolabelles. Il emprunte à M. Cuvier la description de l'animal; mais, voulant justifier la conservation de son genre, il ajoute l'observation suivante: Quie peut-être les Dolabelles se distinguent des Aplysies par le défaut d'yeux, s'appuyant pour ce fait sur ce que M. Cuvier, en décrivant les mollusques rapportés par Péron, n’a point parlé de ces organes. Il est facile 'd'en concevoir le motif : c'est que cet anatomiste s'est particulièrement attaché à décrire ce qui pouvait établir quelque différence entre les denx genres, ajoutant, pour tout le reste, que ces animaux sont parfaitement semblables. Il entendoit donc par là que les Dolabelles portent les yeux comme les Aplysies. Sans doute les recherches de ce savant sont trop rigourenses et trop délicates pour qu'il ait oublié de s'assurer de ce fait; et, dans le cas où il eût reconnu une particularité si remarquable, il n'est pas à croire qu'il eüt négligé d'en faire mention. M. de Lamarck ne décrit que deux espèces dont une est nouvelle. Il renvoie à M. Cuvier pour les $D$. Rondeletii et dolabrifera dont nous venons de parler. La nouvelle espèce qui n'est connue que par sa coquille, a reçu le nom de $D$. fragilis; c'est tout simplement le test de l'A. depilans.

M. de Férussac, dans ses Tableaux systématiques, maintient le genre Dolabelle, et y rapporte les trois espèces citées en dernier lieu par M. Cuvier.

M. de Blainville a également cherché à y établir de nouvelles espèces, mais sans beaucoup de succès. Dans le Dictionnaire des sciences naturelles, il en établit deux que nous allons examiner, l'une sous le nom de $D$. Peronii, et l'autre sous celui de $D$. lavis.

Il pense que la Dolabelle rapportée par Péron et figurée dans le mémoire anatomique de M. Guvier, est une espèce distincte de celle représentée par Rumphius; il croit en outre que la coquille de la planche XL de cet iconographe se rapporte à l'espèce de Péron et non au limax marina tertia.

Nous ne saurions partager son avis; il nous paroît certain que ces deux espèces n'en font qu'une, à laquelle la coquille de Rumphius appartient. Tous les caractères du limax marina

(i) Lajurck, Systeme des Anim. sans vert., t. VI, II ${ }^{\mathrm{e}}$ P., p. 40. 
se rapportent à la Dolabelle que l'on doit à Péron, si l'on en excepte des inexactitudes de dessin qu'il est très-facile de reconnoître dans la figure du premier. "La Dolabelle de "Rumphius diffère, dit-il, de celle de Péron par une peau beaucoup plus tuberculeuse, " une moindre grandeur du disque, par la présence de véritables appendices natatoires, " quoique moins grands que dans les Aplysies, et enfin par l'existence d'un tube (le siphon) "plus long à la cavité branchiale.»

Il est vrai que, dans cette figure, le disque offre une moindre grandeur; c'est parce que les bords supérieurs, représentés dans un état de mollesse, sont affaissés, et ce sont précisément ces mêmes bords ainsi affaissés et lâchement repliés et ridés qui donnent l'apparence de lobes natatoires. Il est possible aussi que Rumphius, voulant montrer dans sa figure la disposition de la cavité branchiale, ait voulu qu'on en écartât les bords. Sa peau, continue M. de Blainville, est plus tuberculeuse; c'est que l'individu de Péron, comme il est facile de s'en convaincre par l'examen de la coquille, est jeune; et nous avons remarqué, sur plusieurs espèces vivantes que nous avons eues à notre disposition, que le corps est beaucoup moins hérissé dans le jeune âge que dans l'état adulte. Quant à la longueur du siphon, elle ne peut précisément sérvir de caractère spécifique, à moins d'ètre extrêmement marquée, car ces mollusques peuvent assez facilement, par l'effet de leurs muscles, allonger ou raccourcir plus ou moins cette partie.

L'animal de Rumphius paroît avoir un disque postérieur, avec tous les caractères des Aplysies; donc il doit entrer dans le genre Dolabelle de Lamarck. Il a des aspérités aiguës comme l'espèce observée par Péron et par nous à l'île de France, l'espèce rencontrée à Waigiou et Rawack par MM. Quoy et Gaimard, et il vient des mêmes mers. Enfin la coquille figurée dans le même ouvrage, vient du même pays que l'animal, et elle est absolument la même que la coquille trouvée par ces mêmes naturalistes. Peut-on douter que l'espèce ne soit aussi la même?

Au surplus, quelle confiance pouvons-nous avoir dans une figure telle que celle de Rumphius? Que signifient en avant des tentacules antérieurs, très-près de la bouche, ces deux petits tentacules filiformes, sembịables à ceux de certains crustacés? Et ce siphon, peut-on avoir confiance dans ses dimensions, quand sa forme est si inexacte? Pourquoi ce tube conique, à parois continues, au lieu d'être formé par un enroulement de la membrane sur elle-meme? Ces inexactitudes, sensibles à tous les yeux, nous donnent lieu de croire que le dessin de cette figure a été fait de souvenir.

La seconde espèce que propose M. de Blainville, sous le nom de D. laevis, nous laisse dans une plus grande incertitude; elle n'est accompagnée que d'une courte description, très-insuffisante, mais telle cependant que l'on seroit encore tenté de la repousser du genre Dolabelle.

L'auteur de l'article Dolabelle du Dictionnaire classique d'histoire naturelle n'ajoute rien à nos connoissances sur ce genre; il ne fait que répéter d'une manière plus abrégée l'article de M. de Blainville, du Dictionnaire des sciences naturelles. M. Deshayes, sans en faire connoître le motif, rapporte dans sa synonymie le $D$. laevis de M. de Blainville à la $D$. fragilis de M. de Lamarck. Il seroit clair, s'il ne se trompe pas, que le $D$. lavvis seroit tout simplement l' $A$. depilans, ce qui n'est pas probable; car M. de Blainville a vu l'animal de son espèce, et doit-on supposer qu'il ne l'ait pas bien reconnu?

Nos observations sur les Dolabelles, réunies à celles de MM. Quoy et Gaimard et 
Lesson, àchèvent de faire connoître ce mollusque dont M. Cuvier nous a dévoilé l'organisation.

Nous avons vu que la Dolabelle diffère si peu de l'Aplysie, qu'on peut sans inconvénient n'en faire qu'un seul genre; nos observations sur ces animaux nous ayant donné lieu de nous assurer par nous-même qu'en effet ils n'étoient séparés par aucune différence générique, nous les réunissons sous la dénomination qui a été la première connue. Lorsque nous traiterons de la composition du genre Aplysie et de la classification des espèces qui le forment, nous chercherons à justifier cette réunion, selon nous, si nécessaire.

Le genre Notarche, le premier formé après les Aplysies et les Dolabelles, a été établi par M. Cuvier pour un mollusque de l'île de France, et adopté par tous les naturalistes, sur la description et la figure données par ce savant; mais, comme il se trouve placé à peu près dans le même cas que la Dolabelle, nous sommes forcé de le réunir également aux Aplysies : nous en détaillerons les motifs.

La partie historique de la famille des Aplysiens se borne à ce que nous venons de dire, au sujet des genres Aplysie et Dolabelle; car les genres Bursatelle et Actéon, étant plus ou moins nouveaux, très-peu connus, et n'ayant encore éprouvé aucun changement remarquable dans leur placement et leur composition, ils ne laissent que peu de chose à dire : nous donnerons sur ces genres le peu de détails que possède la science, lorsque nous en traiterons méthodiquement à la suite des Aplysies.

\section{ANATOHIE DES APLYSIENS.}

Nous renvoyons pour l'anatomie des A plysiens aux travaux de Bohadsch et de MIM. Cuvier et delle Chiaje, sur le genre Aplysie. Nous aurions pu, en réunissant ce qu'il y a de particulier dans chacun de leurs mémoires, donner une connoissance très-étendue de l'organisation de ces mollusques; mais nous n'avons pas cru devoir le faire, parce que nous n'avons que peu de choses nouvelles à ajouter à ce que nous leur devons, et que d'ailleurs nous avons lieu de croire que le savant à qui la science doit ce qu'elle possède de plus précieux sur les Aplysies, se propose d'enrichir son premier travail de nouvelles descriptions anatomiques qui ne peavent manquer de le rendre aussi complet què possible.

Les Bursatelles de M. de Blainville offrent la même organisation que les Aplysies. Quant aux Actéons d'Ocken, nous n'avons que peu de détails, mais beaucoup de doutes à leur sujet; ce n'est donc que d'après l'analogie qu'ils nous présentent dans leur forme générale, et sur la description très-insuffisante de Montagu, que nous les réunissons à la famille des Aplysiens.

\section{DESGRIPTION EXTÉRIEURE DES APLYSIENS.}

Forme générale. Le corps des Aplysiens est généralement oblong, quelquefois arrondi, toujours bombé en-dessus et aplati en-dessous pour former le pied; la partie antérieure se prolonge plus on moins, selon les espèces, en une sorte de cou que termine la tête; la partie postérieure est aussi quelquefois allongée et pointue comme une queue, d'autres fois 
elle est très-courte et se montre obtuse. On voit par là quel rapport en apparence peut exister entre ces mollusques et les limaces, et pourquoi Élien comparoit le lièvre marin à un limaçon privé de sa coquille.

Cette description de la forme extérieure n'est applicable à l'animal que lorsqu'il est en mouvement, parce qu'il se montre alors dans toute son extension et tout le développement de ses organes; mais ces caractères ne seroient plus reconnoissables sur le même individu lorsqu'il viendroit à se contracter, comme il arrive souvent, soit dans le repos, la souffrance, ou durant l'accouplement. En effet, dans ces.circonstances, l'enveloppe extérieure, qui est soumise à l'action d'une infinité de muscles, prend des formes très-variables, et quelquefois telles qu'au premier aspect on ne reconnoît plus les diverses parties de l'animal: Ce fut sans doute dans un de ces moments que Pline observa le lièvre-marin, puisqu'il le compare à une måsse informe. En général, lorsque les Aplysiens se contractent, ils se gonflent et se raccourcissent; leurs parties antérieures et postérieures rentrent dans la partie moyenne, et les tentacules eux-mêmes disparoissent presque en entier. Le pied prend aussi diverses formes; quelquefois ses bords se perdent dans les inégalités du manteau; d'autres fồs ses extrémités antérieures et postérieures se relèvent, et le mollusque prend à peu près la forme d'une boule, ou bien elles s'abaissent et tendent à se rapprocher, alors il s'élargit dans le milieu.

Il est donc essentiel, pour juger de la forme de ces animaux, de les observer vivants et en mouvement, puisque c'est alors seulement qu'ils montrent, dans tout leur développement, les caractères qui les distinguent. C'est sans doute une attention qu'il est bon d'avoir pour tous les mollusques en général, mais pour les Aplysiens peut-être plus que pour les autres. M. Cuvier, en figurant l' $A$. punctata, a fait voir de quelle manière elle se contracte; il est impossible de mieux exprimer ces variations que ne le font ses figures. L'A: fusca, du Voyage de Krusenstern, a été également représentée dans l'état de contraction: mais cette figure étant unique, il est à regretter qu'on n'ait pas, de préférence, représenté ce mollusque développé.

Il nous paroît aussi très-probable que la figure du Notarchus gelatinosus, dans le Règne animal, ainsi que celles du même Aplysien et de la Bursatelle, dans le Traité de Malacologie de M. de Blainville, ont été faites sur des: individus contractés, et c'est sans doute ce qui leur donne une forme arrondie:

Manteau. Le manteau des Aplysiens est généralement très-vaste; il enveloppe tout le corps en s'élevant sur. le dos. Sa forme varie selon les genres et les espèces; parfois ses bords latéraux, moins dilatés, viennent se rapprocher sur le dos, laissant entre eux une fente étroite longitudinale et presque toujours médiane; d'autres foịs ces mêmes bords latéraux sont très-dilatés, et, en se repliant sur l'animal, ils se croisent l'un par-dessus l'autre : dans ce cas, il se présente encore deux dispositions particulières: ou les côtés de ce manteau, que nous nommons les lobes, sont non-sejlement larges, mais encore longs, et alors l'ouverture dorsale se prolonge beaucoup, surtout en arrière; ou bien ils sont courts et viennent, en se réunissant en arrière du dos, le brider fortement. Dans ces deux cas, les lobes étant toujours.assez amples, ces organes sont propres à la natation. Nous en traiterons plus en détail lorsque nous parlerons de la locomotion chez les Aplysiens.

Le manteau montre encore d'autres caractères, mais qui ne sont que secondaires, et 
propres seulement à la distinction des espèces. Quelquefois, par exemple, il est lisse, d'autres fois il est dur et rugueux; dans certaines espèces il est couvert de petits tubercules arrondis, dans d'autres de nombreuses aspérités aiguës et effilées dont elles sont comme hérissées; enfin il en est dont le manteau est tout couvert d'appendices allongés, grêles, réunis par bouquets plus ou moins fournis et épais à leur base, et qui donnent à l'animal, dans l'eau, l'aspect d'un corps entouré de nombreuses ramifications.

Téte. La tête est arrondie; quelquefois, mais c'est assez rare, elle est séparée du cou 'par un léger étranglement. La bouche est fendue en long, et munie intérieurement de deux pièces cornées, symétriques et brunes. L'une, placée en avant, et formée de deux lames égales, réunies en haut et en bas, en garnit l'entrée et donne passage aux aliments dans son milieu; l'autre, située dans le fond et en haut, est simple, mais pliée en cleux, ct ses faces internes sont munies d'une grande quantité de petites dents en forme de cônes recourbés, toutes rangées dans un ordre extrêmement régulier. La bouche n'est pas susceptible de beaucoup de dilatation.

Tentacules. Les Aplysiens ont deux paires de tentacules : l'une située très-près de la bouche, tout-à-fait à la partie antérieure de là tête. Ces tentacules paroissent formés par le prolongement de la membrane qui environne cette ouverture; c'est pourquoi nous les nommons, avec M. de Blainville, tentacules labiaux; ils sont très-larges, aplatis, et de forme plus ou moins arrondie ou oblongue; ils se tiennent assez généralement enroulés en forme de cornet pendant le repos, et se développent en éventail pendant le mouvement; mais, dans tous les cas, ils se dirigent toujours en avant et sur les côtés. Leurs bords sont quelquefois droits et d'autres fois ondulés; ils sont susceptibles de contractions.

L'autre paire de tentacules, les tentacules postérieurs, est située en arrière de la tête de chaque côté de la partie supérieure du cou. La séparation de leur base varie un peu, et leur forme diffère de celle des tentacules labiaux; ils sont coniques, aigus ou obtus, plus ou moins allongés, roides pendant le mouvement et susceptibles de beaucoup de contraction, mais sans pouvoir disparoître entièrement. On les a souvent comparés à des oreilles de lièvre à cause de leur allongement et de l'ouverture en forme de fente longitudinale qui se remarque à leur partie supérieure. Leur surface ne montre jamais de rides ou plis réguliers, mais seulement ceux qui proviennent des contractions fréquentes.

Les Actéons paroîtroient n'avoir que deux tentacules; c'est même un des caractères qui semblent devoir les détacher des Aplysiens, mais auxquels on ne peut cependant ajonter encore une grande confiance, car il est fort possible que dans ces petits mollusques les tentacules labiaux, plus réduits que dans les autres Aplysiens, aient échappé aux recherches du naturaliste qui les a observés.

Yeux. Il en est de même des yeux: chez les Aplysies, ils sont situés immédiatement en avant des tentacules postérieurs, un peu plus sur les còtés, tandis que dans les Actéons seủlement ils se trouvent en arrière. Cẹs yeux, dans les uns et les autres, sont sessiles, extrêmement petits, et semblables à un point noir ou bleuâtre entouré d'un petit cercle blanc; dans quelques espèces d'Aplysies ils sont à peine visibles.'

Cavité branchiale. La cavité branchiale occupe une grande portion du dos ou de la partie bombée du mollusque; quelquefois elle est située un peu plus en avant, et d'autres fois plus en arrière. L'organe important qu'elle contient est souvent protégé par un opercule qui la ferme plus ou moins complétement, et de plus, par les lobes du manteau, 
soit lorsqu'ils rapprochent leurs bords l'un contre l'autre, soit lorsqu'ils se croisent l'un par-dessus l'autre. Quand l'animal se met en mouvement, les lobes du manteau s'écartent, l'opercule se soulève au côté droit qui est libre, la cavité se dilate considérablement, et le panache des branchies flotte en liberté dans l'élément ambiant. Au contraire, lorsque l'animal ést immobile et contracté, la cavité est resserrée, l'opercule abaissé et les lobes abattus sur lui laissant un passage en arrière par où sort généralement un tube membraneux qui conduit directement l'eau aux branchies. Nous parlerous tout à l'heure de ce tube que nous nommons le siphon.

Branchies. Les branchies, situées dans un plan quelquefois horizontal et quelquefois oblique, d'avant en arrière, ont la forme d'un panache aplati dont le milieu seroit tourné en avant et les pointes dirigéeś de chaque côté; elles sont attachées aux deux faces d'un diaphragme triangulaire qui se porte du bord antérieur de la cavité au bord postérieur. Les lamelles branchiales sont à peu près triangulaires et groupées deux à deux de chaque côté de ce diaphragme; chacune d'elles est, comme dans la plupart des mollusques, subdivisée en lamelles secondaires. Ce panache est fortement recourbé, la partie concave en arrière; l'extrémité de gauche se perd sous l'opercule, et celle de droite, prolongeant le bord de cette pièce, est quelquefois très-longue, acuminée, et alors elle s'échappe de la cavité pendant le mouvement. Les branchies vont en décroissant du centre vers les extrémités, mais la partie de droite est toujours plus longue que celle de gauche.

Opercule. L'opercule est cette pièce dont nous venons de parler, qui protège en premier lieu les branchies dans un grand nombre-d'espèces d'Aplysies; il est de forme oblongue, un peu convexe, libre au côté droit, et en partie en avant et en arrière; il est composé d'une double membrane, et quelquefois d'un rudiment de coquille intérieur qui sert à lui donner plus de solidité. Cette double membrane vient former en arrière et un peu ḋ droite, par un vaste repli enroulé sur lui-même, ce tube que nous appelons le siphon: Cet organe correspond, dans les espèces qui ont un rudiment de coquille, à une échancrure pratiquée au côté droit postérieur. de cette pièce solide. Outre la coquille qui se montre quelquefois dans l'opercule, on y troure encore une glande qui sécrète la liqueur pourprée. Lorsque l'opercule est solidifié par un rudiment testacé, il est généralement assez vaste; mais lorsqu'il n'est formé que par une double membrane, il est alors très-étroit, quelquefois à peine distinct; et dans ce cas, qui est celui du sous-genre Notarche et des Bursatelles, la cavité branchiale est très-resserrée, les lobes du manteau presque nuls, et la fente dorsale très-petite. On conçoit qu'avec une semblable disposition la présence d'un rudiment testacé devenoit inutile. Dans ce dernier cas encore, il n'existe aucun siphon apparent.

Parmi les espèces d'Aplysies qui sont mụnies d'une coquille dans l'intérieur de leưr opercule, on remarque les deux caractères suivants : ou la membrane supérieure de cet opercule porte dans son centre un petit tube conique, membraneux, ouvert à son extrémité; ou bien cette mème membrane est largement ouverte dans son milieu, comme si elle avoit été fendue, et la coquille esț en partie à découvert. Il arrive souvent aussi, comme nous l'avons remarqué dans l' $A$. fasciata qui porte un petit tube, que la membrane se déchire, de manière à former une large ouverture irrégulière qui remplace celle du tube: Nous trouverons peut-être la cause de cet accident dans la connoissance d'un fait assez singulier que nous avons eu souvent lieu d'observer dans les espèces à coquille. 
On doit croire que l'ouverture naturelle de certaines espèces, qu'elle soit simple, ou à l'extrémité d'un tube, est destinée à un usage particulier et de quelque importance dans l'existence de ces animaux. Nous ne saurions dire au juste quel est cet usage, cependant nous avons observé que les espèces d'Aplysies qui montrent cette ouverture ont presque toujours l'opercule plus ou moins chargé, entre le test et la membrane supérieure, de vase et quelquefois même de gravier et de débris de coquillages. Nous avons souvent trouvé $l^{\prime}$ A. fasciata tellement chargée de ces matières que la coquille en étoit toute brisée. Nous pensons donc que la présence dans l'opercule de ces corps étrangers est nécessaire à l'animal, et que leur introduction se fait, selon la grosseur des matières, ou par le tube ou par l'ouverture large dont nous avons parlé, et qui n'ont peutêtre pas d'autre usage. Or, nous croyons reconnoìtre là cause du déchirement de la membrane de certaines espèces, telles que l'A. fasciala, dans la surabondance des matières accumulées dans l'opercule, qui, à force d'en distendre les parois, doit finir par les déchirer.

Peut - être existe-t-il encore quelques espèces ne montrant ni ce tube ni cette oulverture; c'est ce dont nous n'avons pu nous assurer complètement; du reste, les $A$ plysies qui composoient le genre Dolabelle pourroient être seules dans ce cas. L'opercule des espèces qui sont privées d'un rudiment testacé ne nous ayant pas offert assez de prise à l'observation, nous ne pouvons affirmer si elles ne possèdent pas aussi l'un de ces deux caractères, ou au moins quelque chose d'analogue. Quant aux Actéons, nous ignorons encore comment sont disposés leurs branchies, et par conséquent si ces mollusques sont munis d'un opercule; Montagu ne nous apprend rien sur ce point important, sans la connoissance duquel il est impossible de placer ce genre d'une manière convenable.

Anus. L'anus est placé en arrière des branchies. Dans les espèces qui montrent un siphon, il est à la base intérieure de cet organe, dans lequel on l'aperçoit facilement lorsqu'il vient à se dérouler.

Organes de la génération. Les organes de la génération sont séparés sur le même individu. L'orifice des oufs s'ouvre un peu en avant et à droite du bord antérieur de l'opercule. Une rainure ou canal très-étroit s'en échappe en parcourant la surface extérieure du moliusque, prolonge le côté droit du cou, et va se terminer sous le tentacule droit antérieur où est situé l'orifice de l'organe mâle. Ce canal, qui est d'une grande importance dans l'acte de la génération, est bordé de chaque côté par un étroit repli de la peau. Dans quelques mollusques Aplysiens il est médian pendant un premier espace, puis il se porte antérieurement à droite; dans d'autres il se porte de suite à droite en quittant la vulve. Les espèces qui n'ont point de lobes latéraux dilatés, sont dans le premier cas, et celles qui en sont inunies sont dans le second. La verge est épaisse et cylindracée à sa base; elle se prolonge ensuite en une sorte de pointe très-effilée et marquée sur le côté d'une rainure qui est le prolongement de celle qui vient de la vulve. Elle est très-contractile, et rentre dans une sorte de gaine membraneuse, épaisse et généralement brune. La verge est d'un beau jaune.

Cet organe est constamment retiré dans le corps, et ne sort que pour l'accouplement: cependant M. Cuvier a remarqué, et nous avons quelquefois fait la même observation, que, lorsqu'on faisoit périr les Aplysies, soit en les plongeant dans l'esprit-de-vin, soit en les mettant dans l'eau douce, il se portoit tout à coup en dehors. 
Pied. Le pied des Aplysiens est grand et oblong; sa largeur. varie beaucoup dans les espèces; la membrane qui le revêt n'est guère plus épaisse ni plus rude que celle du reste du corps, sans doute à cause du peu d'usage que ces mollusques en font. Ses bords ne sont pas toujours très-distincts, car sa surface se lie immédiatement à celle du manteau; aussi est-il soumis à toutes les contractions de cette enveloppe, ce qui le fait considérablement varier dans sa forme et ses mouvements. Nous en parlerons plus én détail lorsque nous traiterons de la locomotion.

Couleur des Aplysiens: Si l'on considère la faculté que possèdent les Aplysiens de se contracter et de se montrer sous les formes les plus irrégulières en mềme temps que nombre d'entre elles répandent avec abondance différentes liqueurs, on concevra facilement tout ce que ces animaux ont de repoussant dans leur aspect, et pourquoi les anciens avoient conçu une si grande horreur du lièvre-marin; mais il est beaucoup d'espèces qui ne sont point aussi disgraciées de la nature, et dont la variété et l'arrangement des couleurs qui ornent le manteau rendent l'aspect moins désagréable. L'A: longicauda, par exemple, se distingue par une belle couleur verte, dont l'uniformité est interrompue par des taches d'un beau rouge entourées d'un cercle bleu : notre petite Aplysie citrine, de même que l'Actéon vert, n'offrent rien que d'agréable aux yeux par le. vif éclat de leur manteau, et les Aplysia Teremidi et Protea, singulièrement variées dans leurs couleurs et leurs nombreux anneaux, font oublier, par la richesse de leur robe, ce que leur forme peut avoir de hideux et de dégoûtant.

La couleur di manteau des Aplysiens est remarquable en ce qu'elle n'adhère pas si intimement à l'épiderme qu'elle ne puisse s'effacer par le simple frottement. Cette particularité existe chéz beaucoup de mollusques, mais plus visiblement peut-être chèz les Aplysies, parmi les Gastéropodes et chez les Séches, les Calmars, etc., parmi les Céphalopodes. Il nous est souvent arrivé, en passant doucement et à plusieurs reprises le doigt sur le corps de l'A. fasciata ou de l'A. dactylomela, de délayer leur couleur, et de mettre ainsi à découvert la blancheur livide de leur épiderme.

Nous ne sommes pas non plus éloignés de croire que d'autres' causes que celle du frottement peuvent quelquefois produire cet effet, et la raison en est que l'on trouve souvent des individus vivants et entièrement décolorés. On ne peut croire que ces individus, battus par les flots de la mer sur les rochers ou le sable, aient souffert un frottement qui auroit détruit jusqu'au dernier vestige l'éclat de leur robe. Il est plus naturel, ce nous semble, de rapporter ce fait à d'autres causes. L'âge ou l'état de maladie ne peuvent-ils rien produire de semblable? ou bien n'est-il pas plus justé de croire qu'à certaines époques ces mollusques éprouvent un changement dans leur couleur, et peut-être dans leur épiderme, comme on le voit chez beaucoup d'autres animaux d'un ordre supérieur?

D'après cela, on conçoit que si l'on ne met pas la plus grande attention dans l'observation des espèces que l'on rencontre, on peut tomber dans de graves erreurs; car on pourroit quelquefois prendre pour deux espèces différentes deux individus de la même, mais dont l'un auroit perdu ses couleurs, tandis que l'autre les conserveroit. Cette observation fera du moins sentir combien il est urgent d'établir les distinctions spécifiques sur des caractères plus importants et plus immuables que ceux qui proviennent du choix et de l'arrangement des couleurs.

S'il est vrai que certains animaux prennent parfois les mêmes couleurs que les 
objets an milieu desquels ils vivent ou se nourrissent, c'est surtout parmi les Aplysiens qu'il est facile d'en faire la remarque. Un grand nombre d'espèces sont de la couleur verte des fucus au milieu desquels elles habitent; l'A. citrina, vivant sur le fucus natans, qui est d'un beau jaune, est de cette même couleur; sa taille, sa forme et les petites taches dont elle est recouverte, lui donnent l'aspect d'une feuille de cette plante ( $\mathrm{I}$ ) : aussi est-ce au hasard que nous devons de l'avoir trouvée; car notre oeil n'auroit pu que très-difficilement la distinguer parmi le feuillage qui lui servoit d'asile. L'A. maculata, que nous avons recueillie au cap de Bonne-Espérance parmi des fucus d'une couleur roussâtre, nous a offert un manteau de couleur rousse: enfin, l'exemple de l'A. dolabrifera nous présente quelque chose de plus extraordinaire encore;'cette jolie et curieuse espèce vit dans des eaux saumâtres, dans le voisinage de la mer et sur un fond de vase. Les seuls mollusques que nous avons trouvés avec elle dans ces endroits sont une Pintadine, qui est un mollusque marin, et deux Néritines, le $N$. auriculata et le $N$. zigzag, appartenant toutes deux aux eaux douces. Ce que nous voulons faire remarquer ici, c'est que le manteau de cette Aplysie a précisément la même couleur, et présente les mêmes dessins que les manteaux de ces différents animaux. Nous pourrions, en examinant attentivement chaque espèce d'Aplysies, faire la même remarque : ne devons-nous pas voir dans ce phénomène l'influence secrète de la nourriture, des propriétés des lieux et de tous les principes environnants, ou, pour mieux dire, la sage prévoyance de la nature, qui a voulu donner à ces foibles animaux un moyen d'échapper à leur ennemi en les confondant avec les objets qui leur servent de refuge?

\section{OBSERVATIONS GÉNÉRALES SUR LES ORGANES ET LES FACULTÉS DES APLYYSIENS.}

Sens. Nous ne savons que bien peu de chose sur les sens des Aplysiens, comme sur ceux des mollusques en général. Nous ignorons complètement si ces animaux possèdent ceux de l'ouie et de l'odorat; quant an tact, au goût et à la vue, nous ne saurions en douter.

Lc tact est leur sens par excellence; il n'a pas seulement pour organes les tentacules, il se manifeste encore dans toutes les parties de leur corps, qui est d'une sensibilité extrême. $\mathrm{Au}$ moindre contact, on voit ces animaux se-contracter. Bohadsch, en parlant de l'organe mâle des Aplysies, rapporte un fait qui prouve jusqu'à quel point est poussée la force d'irritabilité de ces mollusques. Il dit que l'animal venant de mourir et le pennis étant même arraché, cet organe se contractoit sitôt qu'on venoit à le toucher. Nous avons plusicurs fois cherché à faire cette expérience, et nous nous sommes convaincus de son exactitude d'une manière assez satisfaisante, mais dans un seul cas, celui où nous avions surpris l'individu pendant l'accouplement.

Le sens de la vue doit exister chez les Aplysiens, puisqu'ils ont des yeux; mais il est probablement bien borné, car ces organes sont extrêmement petits.

On doit croire aussi qu'ils possèdent celui du goût; l'organisation compliquée de la bouche et la variété des substances dont ils se nourrissent semblent en être la preuvè.

(1) Les mollusques qui habitent avec elle sur le fucus natans, sont des Scyllées, qui se rapprochent encore plus, s'il est possible, par leurs conleurs et la forme de leurs palettes branchiales, de la couleur et de la disposition des feuilles et des fruits de cette plante errante. 
Quant à l'ouie et l'odorat, ils ne se manifestent par aucun organe apparent à nos yeux, á moins que, partageant l'opinion de M. de Blainville, nous ne placions le siége de l'odorat dans les tentacules supérieurs: mais, sur une telle matière, une simple opinion est-elle bien suffisante? n'est-on pas en droit de demander, sinon des preuves, au moins des présomptions puissamment fondées? et nous n'en trouvans aucune bien propre à justifier la pensée de ce savant.

Organes locomoteurs. Les Aplysiens sont mieux doués du côté des organes locomoteurs que beaucoup d'autres mollusques. Ils possèdent, comme tous les Gastéropodes, un pied pour ramper; mais ils ont en outre des organes natatoires, analogues à ceux des Céphalopodes et des Ptéropodes: cependant tous ne sont pas également bien organisés sous ce dernier rapport; car il en est chez qui les membranes, destinées à la natation, sont tellement étroites et serrées sur le dos, qu'elles ne peuvent en aucune façon servir à les tenir suspendus sur les eaux.

Quand on embrasse d'un seul coup-d'œil toutes les espèces qui composent nos différentes sections du genre Aplysie, on ne peut manquer de reconnoître encore cette industrieuse prévoyance de la nature qui a si bien su conformer tous ces animaux aux localités qu’ils sont appelés à occuper, ainsi qựaux habitudes qui résultent de celles-ci. Par exemple, les espèces qui forment la première et la seconde section n'habitent que dans les mares fangeuses et sur des rives vaseuses et unies; elles ont, pour s'y mouvoir, un pied très-grand, propre à embrasser une grahide surface, et leurs lobes serrés sur le dos et en même temps très-étroits ne leur permettent pas de nager. Les espèces de la troisième section, appelées à vivre dans les excavations des rochers, au fond des baies et dans des lieux moins tranquilles, sont un peu différemment organisées; leur pied, toujours large, est susceptible de varier considérablement ses formes, de manière à ramper plus facilement sur un sol inégal, et les lobes du manteau, acquérant une ampleur considérable, se rabattent, à la volonté de l'animal, sur les côtés, et, faisant l'office de véritables nageoires, se portent au milieu des eaux et au-delà d'espaces quelquefois trèsgrands. Enfin, les espèces qui forment notre sous-genre Notarche, montrent encore une différence bien sensible; elles paroissent, autant que nous pouvons en juger en ce moment, d'après la connoissance d'un petit nombre d'espèces, habiter de préférence sur des fucus, avec lesquels elles se laissent entrainer à de grandes distanees en mer, comme MM. Quoy et Gaimard l'ont observé sur l'A. longicaüda, et comme nous l'avons nousmêmes remarqué pour l'A. citrina; leur pied a dû nécessairement subir une modification appropriée à cette nouvelle destination, et nous remarquons en effet qu'il est plus étroit, plus allongé, comme pour embrasser plus commodément les branches et les feuilles des plantes sur lesquelles elles fixent leur séjour.

Toutes ces différences d'organisation ne sont que des modifications plus ou moins grandes des caractères du genre; mais ces modifications, comme nous aurons lieu de le voir, nous fournissent des caractères pour la réunion en groupes distincts des espèces que nous sommes parvenus à distinguer, et pour l'arrangement de ces mèmes groupes.

Le pied des Aplysiens, quelles que soient son étendue et sa forme, n'est pas toujours trèsdistinct du manteau de l'animal, surtout sur les côtés; car à la partie antérieure, il est complètement séparé de la tête par un enfoncement. La membrane qui le revêt n'est pas 
beaucoup plus épaisse que celle qui recouvre le corps; mais elle est susceptible, comme nous l'avons déja dit, de contractions considérables qui en font beaucoup varier la forme. Il offre sur le même individu tantôt des rides nombreuses, tantôt des cavités profondes, èt tantôt des callosités. Sa couleur est toujours celle du manteau, mais elle est plus pâle; ses bords se garnissent quelquefois, de boursouflures variées dans leur forme et leur arrangement, mais qui ne sont produites que par les contractions et ne peuvent par conséquent fournir de caractères constants. Dans la seconde section de nos Aplysies, ce pied se montre plus large à proportion que daus les autres, parce que les animaux qui la composent ont le manteau très-dilaté à leur base, ce qui fait que, vus en dessus, ils paroissent envirounés d'une large expansion membraneuse, très-mince sur ses bords, et qui ne se remarque point dans la première section non plus que dans les Notarches. On retrouve encore quelques vestiges de cette disposition du pied ou plutôt des bords latéraux inférieurs du manteau, dans certaines espèces de la troisième; mais cela vient souvent de la facilité avec laquelle ces sortes d'animaux peuvent changer la forme de leur enveloppe extérieure.

Lorsque les Aplysiens sont immobiles sur les rochers, ils s'y tiennent quelquefois fortement attachés par leur pied, comme on le voit chez la p'lupart des Gastéropodes, en se contractant et faisant le vide; c'est par ce moyen qu'ils résistent à la vague qui les heurte, et qui sans cela les entraîneroit sur le rivage.

Lorsque ces animaux se mettent en mouvement, ils se développent en entier, donnent tonte l'extension possible à la surface de leur pied, et rampent avec une extrême lenteur, de'manière que les mouvements de cet organe sont à peine sensibles à l'oeil. Pendant cette action, le cou allongé se redresse et se porte en avant et sur les côtés; les tentacules s'allongent et se dirigent sur différents points avec beaucoup de mobilité; la partie postérieure du manteau et du pied se prolonge par derrière; et les lobes latéraux, lâchement rabattus sur la cavité branchiale, laissent sortir l'extrémité du siphon, qui se dilate pour recevoirl'e au nécessaire aux organes avec lesquels il communique.

Les lobes natatoires des Aplysiens sont deux expansions plus ou moins considérables des bords supérieurs du manteau, les mêmes qui, dans beaucoup de mollusques gastéropodes, tels que les Cyprées, les Parmophores, etc. viennent quelquefois recouvrir le dessus de leur enveloppe testacée. Ces lobes, placés latéralement, occupent une grande partie de la longueur de l'animal, se croisent sur son dos pendant le repos, sont de forme demi-circulaire, et montrent facilement à l'oeil, surtout en dedans, le tissu croisé de filets musculaires dont ils sont si abondamment chargés.

M. de Blainville a signalé comme caractère dans le genre Aplysie, la différence que l'on remarque quelquefois dans la largeur de ces deux lobes sur un même individu de certaines espèces. Nous ne considérons point ce fait, que nous sommes cependant loin de contester complètement, comme pouvant fournir un caractère constant. Selon nous, cette différence est quelquefois accidentelle, quelquefois peut-être momentanément apparente, et jamais assez marquée pour que l'on puisse la saisir avec facilité. Enfin, si elle existe dans quelques individus, nous ne dirons pas dans quelques espèces, cela ne peut-il pas venir de ce que ces animaux contractent l'habitude, dans ce croisement des lobes, de placer toujours le même par-dessus, le supérieur pouvant alors acquérir, avec le temps, plus de développement que l'inférieur qui se trouve en partie comprimé? 
Les Aplysiens, comme nous l'avons déja dit, ne montrent pas tous des lobes natatoires. Dans le genre Aplysie, la troisième section seule en est munie; les Bursatelles en paroissent privées; mais ces organes reparoissent de nouveau chez les Actéons. Ceux qui en ont s'en servent avec assez d'agilité, en les abattant de chaque côté du corps, dans un plan horizontal, et les relevant alternativement. Dans cet instant, le corps prend toute l'extension dont il est susceptible; la cavité branchiale, entièrement à découvert, laisse sortir le panache des branchies qui saillit plus ou moins en dehors, selon sa longueur; le pied se contracte transversalement, de manière à présenter le moins de surface possible; les tentacules labiaux se déroulent en éventail et offrent de chaque côté de la tête une large surface, au moyen de laquelle ils soutiennent la partie antérieure du cou et aident peut-être au mouvement, sans pour cela déroger à leur principale fonction, celle de pouvoir, en se portant en avant, prévenir un choc inattendu.

M. de Blainville pense que les Aplysies nagent le dos en bas : nous n'avons jamais pu.nous assurer de ce fait, ne les ayant jamais vues nager que dans le sens naturel où on les voit ramper, et nous sommes très-porté à croire d'ailleurs qu'elles ne font pas autrement; nous admettrons peut-être plus facilement avec M. Cuvier qu'elles peuvent venir se suspendre à la surface des eaux, comme certains mollusques d'eau douce, en lui présentant le pied; cependant nous avons vainement cherché à les surprendre dans cette position.

M. Cuvier, qui a si bien examiné ces animaux dans l'état de vie et pendant leurs mouvements, ajoute qu'ils nagent par des ondulations successives : nous avons effectivement eu lieu de voir qu'ils ne se bornoient pas à se servir de leurs lobes pour se mouvoir sur les eaux, mais qu'ils accéléroient encore leur marche par des mouvements ondulatoires dans le.sens latéral ; on conçoit de quel avantage doit être cette nouvelle ressource pour les espèces allongées.

Sécrétions. Les Aplysies épanchent trois sortes de liqueur. La première suinte de toutes les parties du manteau; la seconde est sécrétée par une glande particulière qui avoisine l'orifice des oeufs, et la troisième par des glandes renfermées dans l'opercule; celle-ci est la plus abondante.

La liqueur sécrétée par le manteau a été observêe pour la première fois par Bohadsch, et il est toujours facile de la reconnoître sur les espèces de nos côtes, qui sont généralement d'une dimension assez grande; c'est surtout lorsqu'on irrite l'animal qu'elle s'échappe par tous les pores et bientôt le recouvre en entier; elle est incolore, limpide, et devient plus abondante, plus épaisse, glaireuse et filante, lorsqu'il se contracte par l'effet de la douleur ou bien lorsqu'on l'irrite. Dans quelques espèces, telles que l'A. depilans et l'A. fasciata, elle est empreinte de l'odeur générale du mollusque. L'épanchement de cette liqueur contribue beaucoup à donner aux Aplysies, lorsqu'elles sont retirées de l'eau, cet aspect repoussant dont nous avons déja parlé.

La seconde liqueur est blanche, épaisse, peu abondante, âcre et fortement odorante; c'est elle qui a été signalée par plusieurs auteurs comme étant venimeuse. Elle sort par une ouverture située en arrière et un peu à droite de l'orifice de la vulve et qui conduit dans une petite masse réniforme que M. de Blainville croit être l'appareil de la dépuration urinaire. On ne sait point au fait à quel usage elle est destinée. Cette liqueur ne paroît se porter à l'extérieur que lorsque l'animal est tourmenté ; du reste on ignore 
encore si elle appartient à toutes les espèces, car nous en avons rencontré qui n’en paroissent pas avoir.

La troisième liqueur dont nous avons à parler est la plus abondante; elle est moins épaisse que les autres et d'une belle couleur de laque: il nous a cependant semblé qu'elle étoịt jaune dans l' $A$. citrina. Les glandes qui la sécrètent sont dans l'opercule mème, et selon l'opinion de MI. Cuvier, qui n'a pu parvenir à leur reconnoître d'issue particulière, elle suinteroit à travers la membrane operculaire. Lorsque l'animal la répand, c'est toujours avec force et abondance; elle se divise aussitôt dans l'eau et forme instantanément un nuage autour de lui. Les anciens n'ont point parlé de cette liqueur, sur laquelle nous reviendrons en parlant des propriétés des Aplysies.

Nous ignorons complètement si les Bursatelles et les Actéons répandent des liqueurs analogues à celles dont nous venons de parler. Il est cependant probable qu'ils possèdent celle qui suinte du manteau; quant à la liqueur colorée, nous croyons au contraire qu'ils en sont privés, à cause du peu de développement que doivent avoir les glandes propres à sa sécrétion, si mêmé ils en possèdent, n'ayant point d'opercule ou ayant tout au plus un rudiment membraneux de cet opercule.

Moyens de défense. Les Aplysiens ne possèdent que de foibles moyens de défense: cependant, s'il est vrai que quelques espèces portent en elles une vertu malfaisante se manifestant au dehors par l'humeur blanche, vireuse et odorante dont nous venons de parler, ce seroit bien certainement là le moyen dont la nature auroit voulu se servir pour mettre à l'abri des attaques de leurs ennemis leur corps si foible, et que ne protègent ni le secours d'une enveloppe solide ni la faculté d'une fuite prompte; mais nous sommes convaincu que, s'il est des espèces malfaisantes, ce n'est qu'en très-petit nombre. Le moyen dont elles paroissent généralement se servir, c'est de répandre autour d'elles la liqueur colorée qui, obscurcissant l'eau, peut les soustraire un instant à toutes recherches; c'est ainsi que plusieurs autres mollusques, les Sèches parmi les Céphalópodes et les Clios parmi les Ptéropodes, parviennent à écarter les animaux qui les inquiètent.

Sans doute ce moyen de défense n'appartient pas à tous les genres d'Aplysiens; il paroît être seulement affecté aux Aplysies; encore est-il probable que les espèces qui forment le sous-genre Notarche en sont également privées; ou du moins si elles possèdent les glandes de cette liqueur, ce ne doit être que pour en répandre une bien petite quantité.

\section{REPRODUCTION, ACCOUPLEMENT.}

Le phénomène de la reproduction chez les mollusques est certainement ce qui mérite, dans l'état actuel de la science, toute notre attention et les recherches les plus soignées. De nombreuses difficultés ont empêché jusqu'ici que cette connoissance ne s'élevât au niveau des autres; cependant d'heureuses observations sur quelques animaux qui vivent près de nous ont répandu quelques lumières dont l'analogie a pu servir, quoiqu'elles soient encore bien insuffisantes. Les Aplysiens n'ont fourni aucun document pour concourir à ce perfectionnement, peut-être parce qu’ils ont été plus généralement négligés par les naturalistes, peut-être aussi parce que les circonśtances qui concourent à l'acte de la reproduction chez ces mollusques se couvrent presque toujours d'un voile mys- 
térieux. Des observateurs tels que Bohadsch et MM. Cuvier et delle Chiaje qui ont observé les Aplysies à l'état de vie, étoient bien faits sans doute pour approfondir ce sujet, si le hasard les eût favorisés; nous ne trouvons rien dans leurs livres qui nous instruise sur cette matière; ainsi nous ne ferons que citer ce que nous avons observé nous-même.

Bohadsch, voulant faire des recherches sur l'accouplement des Aplysies, en conserva quelques-unes pendant plusieurs jours dans un vase rempli d'eau; mais il ne les vit point se réunir, sans doute à cause de l'état de captivité dans lequel ces animaux se trouvoient: cependant, considérant de quelle manière sont placés chez eux les organes de la génération, il pensa, et depuis M. de Blainville a cité son opinion, qu'ils étoient obligés, pour s'accoupler; de se placer tête à queue, afin que les sexes différens fussent en rapport, et conséquemment que l'accouplement fût double. Cette idée, qui est une conséquence naturelle de l'examen de leur organisation extérieure, a dû êtré également la nôtre, avant qu'une heureuse rencontre vînt nous prouver qu'il n'en étoit pas ainsi. Nous parcourions à marée basse les débris de l'ancienne digue de Richelieu, àl'entrée du port de La Rochelle; là ces animaux se montrent quelquefois en nombre considérable, cherchant un abri dans les creux des rochers où la mer a laissé quelque peu d'eau. Sous une pierre déja revêtue à sa face inférieure de plusieurs pelotes diversement colorées d'œufs d'Aplysies, nous trouvâmes accouplés deux gros individus de l'Aplysia fasciata. Notre premier soin fut d'en prendre un dessin (planche VII), puis nous examinâmes, avant que d'y porter la main, de quelle manière s'opéroit leur réunion. Les deux índividus en partie contractés étoient placés l'un contre l'autre, la tête tournée du même côté, celui de gauche étoit seulement un peu plus en arrière que l'autre, en même temps qu'il étoit en avant un peu élevé sur la partie antérieure de son dos. Par cet arrangement la verge du premier correspondoit à la vulve du second, et les contractions de l'un et de l'autre suffisoient pour rendre le rapprochement de ces parties encore plus complet; cependant un espace de quelques lignes laissoit voir la base de la verge dont la partie effilée, que nous avons trouvée ensuite longue de deux pouces environ, étoit plongée tout entière dans le canal des óufs. Cet organe paroissoit avoir pris un développement extraordinaire, et l'orifice de la vulve très-dilaté montroit une sorte de bourlet. Tant qu'a duré cet accouplemẹnt, c'est-à-dire cinq quarts d'heure depuis le moment où nous l'avons aperçu, ces animaux sont restés dans une immobilité parfaite qui attestoit l'état de torpeur dans lequel ils étoient plongés. Une humeur épaisse et glaireuse suintoit abondamment de leurs corps, et leur pied sembloit fixé au rocher. Enfin les premiers flots de la mer montante vinrent les surprendre dans cet état, et c'est alors seulement que nous pûmés les voir remuer quelques parties de leur corps, et sortir de leur engourdissement. Ciraignant.de les perdre, et voyant qu'ils commençoient à s'écarter l'un de l'autre, nous les arrachâmes du rocher, et les ayant séparés, nous pûmes juger de la longueur de la verge qui bientôt après se contracta, tandis que de son côté la vulve se refermoit.

Après la séparation des deux individus, notre premier soin fut de faire mourir celui des deux qui avoit rempli les fonctions de femelle, et d'ouvrir les canaux de la génération, nous attachant surtout à reconnoître le cordon qui vient de l'oviducte et que M. Cuvier signale comme ayant les parois plus épaisses; nous le trouvâmes facilement, car il étoit très-dilaté, sans doute par l'introduction de la verge. Il se montroit en outre muni, dans 
le fond et en petite quantité, d'un produit muqueux que nous n'avons pas balancé à regarder comme étant la semençe qui venoit d'y être déposée.

Nous concluons de ces observations,

$\mathrm{J}^{\circ}$ Que l'accouplement des Aplysies peut être simple.

Nous n'affirmons pas qu'il ne soit jamais double, quoique depuis nous l'ayons toujours vu simple. Peut-être la façon dont ces animaux s'accouplent dépend-elle de la manière dont ils se rencontrent.

$2^{\circ}$ Que la semence s'échappe lentement de l'orifice de la vulve de l'individu qui remplit les fonctions de mâle, parcourt la rainure de son cou, et qu'au moyen de celle qui lui correspond sur toute la longueur de la verge, elle s'introduit ainsi dans le cordon de l'oviducte. On concevra facilement cette marche de la semence, si l'on jette les yeux sur la figure que nous venons de citer, où nous avons représenté, pour plus de facilité, ces animaux moins contractés et plus éloignés au point de réunion des sexes qu'ils ne le sont réellemènt dans l'état d'accouplement. La violente contraction qu'éprouve l'individu de gauche, surtout au cou, raccourcit considérablement le canal qui lie la ygulve à la verge; en même temps il s'élargit, et, selon toute probabilité, les deux replis qui le bordent dans.toute sa longueur, se renversent sur lui, de manière à donner plus de sûreté à la circulation de la semence. La position élevée du mollusque de gauche facilite encore la marche de ce produit, qui n'a plus besoin, pour ainsi dire, que de tomber dans l'orifice dilaté de la vulve. Peut-être que la même facilité n'existeroit pas si l'accouplement étoit double.

Il nous semble maintenant avoir répondu à la question de M. Cuvier, lorsqu'il dit : "Cette rainure sert-elle à conduire la liqueur séminale d'une Aplysie dans le corps de l'autre? Mais nous répéterons avec lui : Pourquoi une telle rainure n’existe-t-elle pas dans tant d'autres' gastéropodes qui n'ont pas non plus de communication intérieure entre leur verge et leur testicule?"

Le produit de la' génération chez les Aplysies n'a pas encore été signalé; cependant 11. delle Chiaje pense que ce peut être ce que les pêcheurs italiens nomment vermicelle de mer. Il ne se trompe pas, ét déja depuis long-temps nous avons reconnu pour des œufs 'd'Aplysies ces pelotes diversement colorées qui, par leur manière de se ranger, ressemblent si bien à du vermicelle. Ces oufs avoient été pris autrefois pour une sorte d'Ulve. Dans les mois de septembre, octobre, et même de novembre, on en rencontre beaucoup sur nos rivages, soit attachés aux rochers, soit embrassant la tige ou les feuilles des plantes marines. Ils sont renfermés dans une longue enveloppe cylindrique et transparente, irrégulièrement contournée. Cette enveloppe est quelquefois teinte de pourpre, quelquefois de jaune et de gris. (Voir la pl. VII, fig. 3, pour leur ensemble, et fig. 4, pour leurs détails.)

Lorsque l'A plysie pond, c'est toujours lentement que ces longues suites d'œufs s'échappent de sa vulve; au moyen d'une humeur glutineuse qui les accompagne, elles s'attachent à l'instant même au rocher ou à la plante marine qui les reçoit, et sur lesquels elles forment bientôt une masse assez considérable.

Sur nos rivages, les Aplysies s'accouplent depuis le mois de juin jusqu'au mois de septembre et même d'octobre; la ponte paroît avoir lieu peu après.

Les genres Bursatelle et Actéon ne nous fournissent aucun renseignement sur l'accou- 
plement; nous devons cependant croire que, sous ce rapport, ils ne different en rien des Aplysies.

régions, habitations, Nourriture.

Régions. Les Aplysies paroissent appartenir à tous les pays: en effet, elles sont connues depuis long -temps sur les rivages de l'Europe; elles le sont davantage, il est vrai, sur ceux du midi que sur ceux du nord; mais cela peut venir de ce qu'elles ont été moins recherchées sur ces derniers, où nous savons cependant qu'elles existent.

L'Asie en fournit plusieurs: de ce nombre sont l'A. Rumphii, aux Grandes-Indes, et l' $A$. fusca dans les mers de la Chine.

En Afrique nous remarquons l'A. Savignana sur les côtes d'Égypte, l'A. fasciata sur celles de Barbarie, et l'A. maculata au cap de Bonne Espérance.

L'Amérique en produit également: dans l'Amérique Septentrionale nous trouvons l' $A$. viridis; dans l'Amérique Méridionale, l' $A$. Brasiliana à Rio-Janeiro, et l' $A$. asciferca à Cayenne; enfin les côtes occidentales de ce vaste continent nous fournissent l'A. Lessonï, que nous devons au naturaliste à qui nous l'avons dédiée.

Les rives des continents ne sont pas seules en possession de ces animaux; les archipels de l'Océanie en produisent plusieurs parmi lesquels nous connoissons les $A$. Rumphii, Teremidi, Hasseltii ét ecaudata; aux îles de France et de Bourbon on rencontre les A. Rumphii, tigrina dolabrifera et gelatinosa, et nous savons qu'à Madagascar il en existe plusieurs espèces qui nous sont éncore inconnues. Dans l'Océan, les $A$. Pleii et protea se montrent aux Antilles, et l'A. dactylomela aux îles du cap Vert et probablement aux Canaries.

Il en est d'autres espèces qui, comme nous l'avons déja dit, abondonnées à la merci des eaux sur des plantes errantes, fuient leur patrie et vont peupler à plus de quatre cents lieues de terre ces immenses champs flottants que-les navigateurs rencontrent parfois au milieu des mers; telles sont les $\mathcal{A}$. citrina, longicauda, nudata, etc.

Nous pouvons donc conclure de là que les Aplysies appartiennent à peu près à toutes les régions.

Après ce coup : d'œil sur la dispersion des Aplysies que nous connoissons, sur la surface du globe, peut-on ne pas être frappé de l'étendue que doit avoir ce beau genre? Combien en effet ne doit-il pas exister d'espèces encore inconnues sur les rivages où on ne s'est point encore empressé de les rechercher!

La distribution des espèces que nous venons de citer, dans les différentes régions dé la terre, nous fournit les remarques suivantes:

$\mathrm{I}^{\circ}$ Les espèces de notre première section, qui toutes se rapportent au genre Dolabelle de Lamarck, sont toutes des mers de l'Inde et de l'Océanie; il est probable qu'il n'en existe point sur la côte occidentale de l'Amérique, et nous sommes certains qu'elles ne se montrent point dans l'Océan Atlantique non plus que dans la Méditerranée;

$2^{\circ}$ Celles de notre seconde section sont encore trop peu nombreuses pour que nous puissions établir leur position sur le globe; cependant nous savons qu'elles appartiennent aux mers de l'Inde, à celle de la Guyanne et à la Méditerranée;

$3^{\circ}$ Celles de la troisième section qui paroissent les plus nombreuses, sont de toutes les régions. 
$4^{\circ}$ Enfin les Notarches, qui paroissent habiter de préférence sur les plantes marines, sont celles qui émigrent arec elles et que l'on retroure au milieu des mers.

Il en est donc des Aplysies comme de plusieurs autres sortes d'animaux; elles sont de tous les pays, mais certains caractères de conformation distinguent quelquefois celles qui peuplent uniquement une région du globe. Ainsi dans le genre Hélice, par exemple, nous royons celles que leur forme distingue si bien, et que l'on a nommées Agathines, peupler de préférence le continent d'Afrique.

Habitations. Les Aplysies habitent différents lieux; les unes recherchent les endroits raseux et tranquilles, elles s'y enfoncent à la manière de la Bullea aperta. Dans cet état, le tube des branchies est l'organe qui se montre le premier, car il se porte toujours en dehors, afin de recevoir l'eau nécessaire à la respiration. Ces animaux, qui ne s'éloignent guère du lieu où ils sont nés, ne nagent point, et appartiennent à nos deux premières sections.

D'autres espèces virent sur les rochers, où elles se cachent dans leurs excavations, ou sous les grosses pierres et parmi les varecs; ce sont les espèces de notre troisième section qui, comme nous l'avons vu, ont la faculté de nager. On les rencontre dans les baies, les rades, mème sur les rirages orageux, pendant les mois favorables aux amours et à la ponte; mais, lorsque rient la mauraise saison, elles se retirent dans de plus grandes profondeurs, ou elles restent dans une sorte d'engourdissement.

Enfin les Notarches recherchent de préférence les fucus, ils s'y tiennent fortement attachés au moyen de leurs pieds. Ces espèces ne nagent point, et ne quittent jamais ces plantes. L'analogie que les Bursatelles semblent aroir arec les Notarches nous fait soupçonner que ces mollusques habitent également parmi les plantes marines. Quant aux tctéons, ils appartiennent aux mers d'Europe, où ils vivent sur les rochers comme les Aplysies.

Nontrilure. Plusieurs sortes de productions marines servent de nourriture aux Aplysiens; mais celles dont ils paroissent rechercher plus généralement l'usage ce sont les plantes marines au milieu desquelles ils virent. Les plus tendres semblent être celles qu'ils préfèrent, si l'on en juge par quelques débris intacts échappés à la digestion, que l'on troure souvent dans leurs lonġs intestins. Ils mangent encore de petits Crustacées, des Mollusques tels que des Eolides, des Tergipes, de petits Doris et des Anélides; nous avons même trouré quelquefois dans leur estomac des fragments de coquilles.

\section{PROPRIÉTÉ, UTILITÉ.}

Propriélé. Les peuples de l'antiquité attribuoient au Lièvre marin, comme à tant d'autres sortes d'animaux, des propriétés fort extraordinaires; aussi rien n'est plus absurde que les fables qui nous ont été transmises à son sujet par les anciens écrivains. C'est surtout dans Pline, qui semble s'être attaché à les recueillir toutes, qu'il faut les chercher pour en prendre une juste idée. Nous nous bornerons à citer les principales, pour faire connoître les exagérations auxquelles peurent si facilement conduire la crainte ou l'ignorance, et afin de donner une nouvelle preuve de l'utilité des sciences naturelles, dont la plus belle prérogative est; sans contredit, de dissiper les erreurs, en conduisant directement à la vérité. 
MI. Cuvier, dans son Mémoire sur l'Aplysie, a déja rapporté une grande partie de ces récits singuliers, et nous aurions pu nous dispenser de les répéter ici, si nous n'arions jugé plus convenable de les consigner dans un même ouvrage ąvec tout ce que nous savons sur un genre de mollusque si intéressant.

Le Lièvre marin, au rapport des anciens, possédoit une vertu malfaisante capable de donner la mort; cette vertu s'exerçoit non-seulement sur ceux qui en aroient maangé la chair ou en avoient bu l'infusion, mais encore sur ceux qui le touchoient. La rue même en étoit dangereuse; si une femme enceinte aperceroit un Lièrre marin femelle, il lui prenoit sur-le-champ de tels vomissements et de si fortes contractions des viscères qu'elle avortoit. Comme il est très-rare que Pline ne fournisse pas le remède ou même le préservatif à côté du mal, il s'empresse de donner celui-ci, qui consiste à porter au bras un Lièvre marin mâle desséché. Dans cette application Pline n'est pas plus heureux que dans beaucoup d'autres, car il se présente une grande difficulté, c'est que tous les individus des Aplysies sont hermaphrodites.

La propriété malfaisante que l'on attribuoit au Lièrre marin n'étoit pas moins grande hor's de l'eau que dans cet élément, et la vie de celui qui en étoit malheureusement affecté s'écouloit péniblement dans la douleur; sa présence repoussoit par une odeur infecte; il éprouvoit sans cesse des romissements et de grands déchirements d'entrailles; quelquefois il ne pouvoit plus uriner; enfin nombre d'accidents renoient l'assaillir et consumer une vie dont le terme étoit toujours marqué par l'instant précis de la mort du mollusque qui en étoit la cause. Pline, citant à l’appui Licinius Macer, fait la réflexion que le poison dú Lièvre marin n’agit point dans un temps déterminé, et dont il soit permis de calculer le terme.

On se doute bien que cet auteur, ainsi que Dioscoride, ne manquẻrent pas d'indiquer une foule de moyens curatifs contre ce terrible poison, de quelque manière qu'on en fùt affecté; ils sont en effet très-nombreux, et M. Cuvier fait remarquer que ces écrivains n'ont pas été guidés dans leurs choix par des principes constants; car ils prescrivent également le vin cuit, la mauve, le lait de femme, celui de jument ou d'ánesse, le sang humain, celui d'oie, le suc de cèdre, les os d'äne, le raisin, l'Alisma, le Cyclamen, l'Ellébore noire, les Cancres de rivière, etc., etc.

Pline assure que le Surmulet peut impunément manger du Lièrre marin; cependant il ajoute que ce poisson devient alors flasque et moins agréable, ce qui en diminuoit beaucoup le prix. Athénée dit pareillement que le Scare peut s'en nourrir.

Il est peu de choses, mème parmi les plus malfaisantes, dont les anciens n'aient pas cherché à tirer des remèdes contre les maladies; et il ne paroît pas qu'ils aient été plus heureux dans ceux qu'ils ont cru pouvoir obtenir du Lière marin, que dans ceux qu'ils ont cherché à opposer à son venin : cependant le nombre des premiers n'est pas moins grand que celui des derniers; c'est ainsi, par exemple, que Pline conseille, comme étant d'un effet merveilleux pour les hernies intestinales, un liniment formé avec ce mollusque et du miel; contre les douleurs de la goutte, des frictions sur la partie malade arec sa chair, etc.

De toutes les propriétés particulières au Lièrre marin, celle dont on a le plus parlé, et que les modernes n'ont pas tous osé mettre complètement en doute, c'est celle de faire tomber le poil. Dioscoride dit que, pour s'en servir à cet usage, il convient d'en former un liniment dans lequel on peut, si l'on reut, faire entrer de l'Ortie de mer (Actinia). Son 
sang et son fiel produisent le même effet, quand même l'animal seroit mort dans l'huile. Enfin Pline ajoute à tout cela qu'on se sert efficacement de sa cendre pour empêcher la reproduction des poils inutiles qu'on enlève des cils des paupières : "Les plus petits, "dit-il, sont les meilleurs pour cet usage.»

Il est facile de reconnoître, dans ce que nous venons de rapporter, les exagérations mensongères si ordinaires aux anciens, lorsqu'ils étoient prévenus contre des animaux qui bien souvent, comme le Lièvre marin, étoient fort loin de posséder tout. le pouvoir de nuire qu'on leur attribuoit. Nous avons déja fait remarquer d'où pouvoit venir cette prévention contre les $A$ plysies; l'aspect informe, la nature mollasse, et les liqueurs souvent fétides et toujours gluantes qu'elles répandent, en sont en partie cause; mais ce seroit un tort sans doute que de repousser aveuglément tous les récits des auteurs anciens, et si nous n'admettons pas que le Lièvre marin, que l'on s'accorde à retrouver dans l'A. depilans, soit pourvu d'un poison tellement violent qu'il puisse causer tout le mal que l'on raconte, nous devons du moins admettre que cette espèce peut produire de graves accidents par l'usage que l'on feroit de sa chair ou plutôt des liqueurs dont il est pourvu.

Le témoignage de quelques auteurs modernes vient appuyer l'opinion des anciens. Bohadsch, en particulier, paroît porté à croire une grande partie de ce qui a été dit; il regarde la liqueur blanche comme venimeuse, et assure que chaque fois qu'il avoit manié quelques-uns de ces animaux, ses mains et ses joues enfloient.

D'un autre côté, M. Cuvier, qui a observé plusieurs espèces d'Aplysies, mais non celle des anciens, a reconnu l'odeur vireuse dont quelques - unes d'entre elles sont affectées; du reste, il assure que ces animaux sont très-innocents, et il croit que l'odeur fétide du Lièvre marin a pu donner. lieu aux fables débitées à son sujet. Il paroît cependant, “ajoute-t-il, qu'en Italie, ce pays où l'art des empoisonnements a été pratiqué et raffiné "si anciennement, on faisoit entrer le Lièvre marin dans quelques-uns de ces breuvages «si usités dans les temps de corruption. Locuste l'employa, dit-on, pour Néron; et "Domitien fut accusé d'en avoir donné à son frère. "

Quant à nous, après de nombreuses expériences, nous pensons que certaines espèces, telles que l' $A$. depilans et l' $A$. fasciala, sont susceptibles, par le seul effet de leur odeur, de déterminer des nausées et même des vomissements chẻz quelques personnes. Nous sommes également tenté de croire, quoique ancun résultat décisif ne soit venu confirmer nos présomptions à ce sujet, que la liqueur ou la substance des espèces que nous venons de citer sont dans le: cas de pouvoir causer de plus grands inconvénients, si toutefois on venoit à s'en servir intérieurement; mais nous croyons que, s'il existe des Aplysies malfaisantes, elles le sont beaucoup moins qu'on ne l'a raconté, et en très-petit nombre. C'est ainsi que, dans plusieurs autres genres d'animaux, les Actinies et les Méduses, par exemple, certaines espèces se montrent avec une propriété corrosive, tandis que d'autres en sont entièrement dépourvues.

Quant à la propriété de dépiler, nous la repoussons, avec MIM. Curier et delle Chiaje, parmi toutes les autres fables auxquelles le Lièvre marin a donné lieu. Bohadsch paroît disposé à appuyer l'opinion des anciens à ce sujet; mais ce naturaliste étoit, comme eux, trop prévenu contre ce mollusque, pour que nous puissions ajouter foi à des observations que, sans doute, il n'a pas faites avec toute l'exactitude désirable.

Nous avons touché un grand nombre d'individus vivants de l' $A$. depilans, et nous nous 
sommes mème frotté les mains et le menton avec les différentes sortes de liqueurs qu'ils répandoient, sans pouvoir obtenir de ces essais le résultat si souvent annoncé par des auteurs trop prompts, sans doute, à croire des traditions populaires.

Utilité. Les Aplysiens n'ont encore aucune utilité reconnue, mais il est peut-être permis de croire que l'industrie pourroit retirer quelque avantage de la liqueur violette que plusieurs d'entre eux possèdent en abondance. La couleur de cette liqueur est très-agréable et d'un éclat vif, lorsqu'elle s'échappe du corps de ces Mollusques, mais elle s'altère promptement au contact de l'air et de la lumière. M. Guvier (I) et M. Fleuriau de Bellevue (2); savant distingué autant par ses propres lumières que par le zèle avec lequel il encourage les différentes branches des sciences et de l'industrie, sont les seuls qui, à notre connoissance, aient fait quelques recherches sur ce beau produit si abondant chez un grand nombre d'espèces. "Cette liqueur, dit le premier de ces savants, prend à l'air, en se desséchant, " une belle teinte foncée, comparable à celle de la Scabiosa atropurpurea, et qui ne paroît " pas susceptible d'altération par l'air seul. L'acide nitrique, en petite quantité, lui donne " une teinte plus violette; lorsqu'on en verse beaucoup, il se change en aurore sale. La "potasse lui donne une teinte d'un gris vineux sale. Ces deux réactifs y produisent "beaucoup de flocons blancs. »

Les recherches de M. Fleuriau de Bellevue ajoutent encore de nouvelles lumières. "Cette liqueur, dit-il, est d'un rouge pourpré très-vif; elle passe ensuite, mais seulement «après plusieurs heures d'exposition à l'air, au rouge de vin, et de là au rouge d'ocre " très-pâle. Étendue dans l'eau et soumise à quelques réactifs, elle a présenté les observa" tions suivantes. Les acides acéteux, nitrique, sulfurique et muriatique augmentoient "singulièrement l'intensité de la couleur, et, ce qui est remarquable, la restituoient «même, lorsqu'elle étoit presque entièrement dissipée; mais cet effet n’avoit que quelques " heures de durée, lorsqu'il étoit produit par les acides acéteux et nitrique, tandis que " par l'acide sulfurique il s'est maintenu pendant plusieurs jours d'exposition à l'air et "même au grand soleil. L'acide sulfurique fixoit réellement la couleur.

«Le carbonate de potasse, l'ammoniac et le sulfate d'alumine, au contraire, dimi"nuent sensiblement la vivacité de cette couleur, et la changent en aurore foible. Ne "pourroit-on pas présumer qu'un acide qui s'évapore facilement prédomine dans la liqueur. "que rejettent ces animaux?"

Il est à désirer que de nouvelles recherches sur cette matière colorante viennent indiquer - à l'industrie le parti qu'elle pourroit en tirer. L'abondance avec laquelle certaines espèces qui en sont munies, surtout l' $A$. fasciata, se montrent sur nos rivages de l'Ouest, et notamment de La Rochelle, seconderoit facilement les heureux résultats que l'on est en droit d'attendre. C'est à la chimie à nous éclairer à ce sujet, en s'emparant d'un produit, peut-être très-précieux, que la zoologie s'empresse de lui signaler.

Nous ne connoissons encore qu'un seul exemple de l'utilité que l'homme, habitant des bords de la mer, peut tirer des Aplysies pour sa nourriture. On avoit toujours pensé que l'aspect dégoûtant et l'odeur repoussante de ces animaux devoient être, pour quelques

(I) Annales du Muséum, tom. II, Mém. cit.

(2) De Roissy, Buffon de Sonnini, tom. V, pag. $\mathbf{7} 2$. 
espèces reconnues innocentes, les seuls motifs qui empêchassent d'en faire un pareil usage, et tout nous porte en effet à le croire; cependant M. Lesson a recueilli pendant le voyage de la corvette la Coquille un exemple qui nous présente une grande exception à cette répugnance. Cet habile naturaliste, toujours soigneux de recueillir les faits qui peuvent faire connoître l'histoire des animaux qu'il découvroit, nous apprend que les habitants des îles de la Société, particulièrement de Borabora, mangent une espèce d'Aplysie trèsabondante sur leurs rivages, et à laquelle ils donnent le nom de Térémidi que nous lui avons conservé. Cette espèce, dont il a bien voulu nous communiquer un beau dessin, et que nous avons ensuite retrouvée dans le cabinet d'anatomie du Jardin des Plantes, est une des plus belles, sous le rapport des couleurs du manteau. Les naturels de ces îles ne se donnent même pas la peine de la faire cuire, et la mangent telle qu'elle est en sortant de l'eau; nous devons même croire que ce Mollusque est un mets friand pour eux, car ce ne peut être le besoin qui les oblige à y recourir, dans un pays que l'on sait être si fertile et dont les produits du sol peuvent seuls suffire à la subsistance de ses nombreux habitants. 


\title{
TABLEAU SYNOPTIQUE
}

\author{
DE LA FAMILLE DES APLYSIENS.
}

\section{A. Quatre tentacules.}

Corps limaciforme.

Cavité branchiale presque toujours protégée par un opercule. Bords latéraux du manteau souvent dilatés en nageoires.

Yeux situés à la base et en avant des tentacules postérieurs.
PREMIER GENRE.

APLYSIE, Nobis, Aplysia.

I. S. G. Aplysies proprement dites. 2. S, G. Notarches.

DEUXIÈME GENRE.

\section{BURSATELLE, Blainville, Bursatella.} geoires.

Yeux?

Corps subarrondi.

Cavité branchiale petite et toujours dépourvue d'opercule.

Bords latéraux du manteau serrés et nullement dilatés en nà-

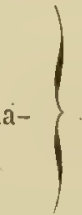

\section{$B$. Deux tentacules.}

\section{Corps limaciforme.}

Cavité branchiale?

Bords latéraux du manteau dilatés en nageoires.

Yeux situés à la base et en arrière des tentacules.

\author{
TROISIÈME GENRE. \\ ACTÉON, Ocken. \\ Actoron.
}

Ce Tableau de la famille des $\boldsymbol{\Lambda}$ plysiens est sans doute bien imparfait, mais cela tient au peu de connoissances que nous avons sur les genres Bursatelle et Actéon; cependant nous avons cru devoir le présenter tel qu’il est, afin de mettre à la fois sous les yeux des naturalistes tous les éléments qui le composent, et les lacunes qui restent à remplir. Ce Tableau se rédniroit encore, si, comme nous sommes tenté de le croire, la Bursatelle n'étoit qu'une espèce du sous-genre Notarche. 


\section{A. Quatre tentacules.}

\section{genre premer. APLYSIE, aPLYSIA, Nobis.}

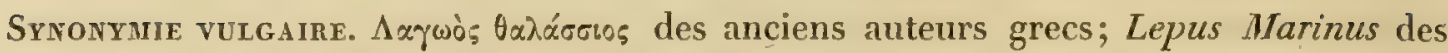
auteurs latins; Lièvre de mer sur les côtes de Provence; Imbriago sur celles du Languedoc; Pichevin (Pisse-vin) sur celles de la Gascogne; Tête-d'âne à Royan; Chat de mer sur les còtes de l'Aunis; Limace de mer sur celles du Nord; Lepre marina des Italiens; Lebre de la mar des Espagnols; Sea heare des Anglais; Baril de vin à la Martinique; Térémidi aux îles de la Société.

Synonyme Scientifique. Lepus marinus, Rondelet, Gesner, Aldrovande, Fabius Columna; genre Lernée, Linné, Syst. nat., $\mathrm{Iv}^{\mathrm{e}}$ et $\mathrm{vi}^{\mathrm{e}}$ édit., id. Bohadsch; genre Thetis, Linné, Syst. nat., $\mathrm{x}^{\mathrm{e}}$ édit.; genre Laplysia, Linné, Syst. nat., xü édit., id. Barbut, Bruguière, Bosc, Lamarck; genre Aplysic, Gmelin, Cuvier, de Roissy, de Férussac, delle Chiaje, de Blainville.

Genre Dolabelle, Lamarck, Cuvier, de Férussac, de Blainville; genre Notarche, Cuvier, de Férussac, de Blainville; genre Actcoon, Ocken, de Férussac.

Corpus repens, oblongum; suprà convexum, infrà planum, anticè elongatum, posticè acutum; duabus membranis lateralibus suprà dorsum reflexis; tentacula quatuor; oculi sessiles anticè, ad basim tentaculorum posteriorum; pecten branchiarum in cavitate dorsi operculo tectä; anus post branchias; vulva anticè ad dextram pectinis, virgaque ad extremitatem anteriorem subtus tentaculum dextrum, canale exteriore ambce conjuncte.

Description. Nous renvoyons à la description des Aplysiens, qui se borne à très-peu de chose près à celle des Aplysies, et dans laquelle nous avons eu soin de distinguer ce qui appartient aux Bursatelles et aux Actéons en particulier.

Histonique. Nous renvoyons également pour l'historique, les mœurs et les propriétés des Aplysies, à ce que nous en avons dit en traitant de la famille des Aplysiens; les détails dans lesquels nous allons entrer sur la manière dont, à notre tour, nous envisageons ce genre, et sur les changements et augmentations que nous y apportons, compléteront la partie historique de ce travail.

Composition vu genre a pLrsie. Le genre Aplysie, tel que nous le présentons aujourd'hui, a toujours pour type l'espèce sur laquelle il a été formé, mais il devient beaucoup plus étendu par suite des nombreuses découvertes qui ont été faites dans ces derniers temps, et comprend des animaux que l'on en avoit à tort séparés par des distinctions génériques. Aucun motif suffisant ne justifiant à nos yeux l'établissement des genres Dolabelle et Notarche, nous avons cru devoir les réunir aux Aplysies, en ayant égard toutefois aux caractères secondaires qui les distinguent de celles-ci, pour former des groupes plus on moins importants dans la série des espèces de ce beau genre.

Nous avons vu que les Dolabelles n'étoient encore connues que par leur coquille, lorsqu'elles furènt établies en genre par ${ }^{\circ} \mathrm{M}$. de Lamarck, mais que bientôt après M. Cuvier, ayant étudié leur animal, avoit jugé qu’il différoit si peu des Aplysies qu'on pouvoit sans inconvénient les réunir dans une mểme description générique. Maintenant 
que l'importance acquise à la famille des Aplysiens par les nombreux matériaux que nous avons rassemblés, exige plus de rectitude et de sévérité dans la composition des genres qu'elle renferme, nous nous étayons à la fois du jugement de ce savant et de nos propres observations pour effectuer la réunion de deux genres dont la grande analogie a été reconnue par tous les naturalistes. Nous allons donner quelques développements aux motifs qui nous décident dans cette circonstance.

Instruit par les recherches anatomiques de M. Cuvier, nous voyons que l'organisation interne de la Dolabelle est jusque dans ses plus petits détails la même que celle de l'Aplysie, à tel point qu'il a regardé comme inutile, après avoir fait connoître celle de cette dernière, d'entrer dans aucun détail anatomique surr la première. Ce n'est donc absolument qu'à l'extérieur que nous devons chercher s'il existe réellement des caractères propres à justifier la distinction établie entre les Dolabelles et les Aplysies; or nous troilvons que toute la partie antérieure de la Dolabelle a la même disposition que dans l'Aplysie; les tentacules sont les mêmes et ne diffèrent tont au plus que dans leurs proportions; les yeux sont également placés à la base des tentacules postérieurs et en avant; la tête et la bouche sont conformées à l'extérieur et organisées à l'intérieur de la même manière; l'orifice des organes de la génération et le canal qui les unit sont semblables, si ce n'est que ce dernier est plus médian en arrière des tentacules postérieurs dans les Dolabelles. Le pied n'offre aucune différence, et s'il en existe quelque part, ce n'est que dans la disposition du manteau. En effet, dans ce dernier genre, il ne montre pas de lobes latéraux, et ses bords sont rapprochés et serrés sur le dos du Mollusque, de manière à ne laisser entre eux qu'une fente étrolte; d'un autre côté; la partie postérieure du corps est obliquement aplatie et présente de cette manière un disque arrondi terminal, au milieu duquel s'arrête la fente dorsale et s'élève le siphon; mais ces deux caractères, que nous regardons comme les plus importants parmi cenx qui sont propres à ces animaux, ne peuvent servir tout au plus, comme nous l'avons déja fait sentir, qu'à former une section dans le genre Aplysie.

Les caractères sur lesquels on paroît s'être le plus appiyé pour justifier l'établissement du genre Dolabelle sont pris dans la coquille; d'abord dans sa nature calcaire, ensuite dans sa forme. On a prétendu que, tandis que celles des Dolabelles étoient calcaires, celles des Aplysies étoient membranenses. Quand cette distinction existeroit, nous ne voyons pas qu'elle soit suffisante; l'exemple de plusieurs genres très-connus vient appuyer cette observation; dans les Sigarets, certaines espèces ont une coquille membraneuse, tandis que d'autres au contraire sont calcaires; nous pourrions en citer encore quelques autres telles que les Bulles, les Hélices, etc. ; mais cette différence n'existe pas ici, ou du moins elle disparoît sitôt que l'on a sous les yeux, comme nous l'avons en ce moment, une suite nombreuse de coquilles de Dolabelle et d'Aplysie. Dans cette suite, sans distinction des deux genres, nous remarquons que ces coquilles sont, à peu d'exceptions près, plus ou moins calcaires et plus, ou moins membraneuses, mais de telle manière que l'une de ces deux natures l'emporte toujours sur l'autre. Si nous examinons d'abord la tête de cette série (sous-genre Aplysie proprement dite, première section), nous remarquons les coquilles des Dolabelles; elles sont très-calcaires, et la partie membranense est réduite à un simple épiderme fort mince; quittant celles-ci, nous rencontrons (même sous-genre, ${ }_{2}{ }^{\mathrm{e}}$ section) des coquilles tout aussi calcaires que ces premières, 
et parmi lesquelles quelques-unes seulement commencent à avoir la partie membraneuse plus épaisse et plus étendue; après ces dernières, il s'en présente de nouvelles (même sous-genre, $3^{\mathrm{e}}$ section) dont la couche calcaire, toujours très-apparente, a perdu de son épaisseur et de sa solidité, tandis que la partie membraneuse devient plus considérable et plus forte; enfin à la suite de celles-ci, cette couche devient à peine sensible, et dans un très-petit nombre d'espèces, peut-être mal connues (fin de la même section), elle semble disparoître en entier. Si nous poursuivions ces observations jusqu'au sous-genre Notarche, nous verrions cette marche décroissante se soutenir, car la coquille, de simple pellicule membraneuse qu'elle paroissoit à la queue des Aplysies proprement dites, devient nulle dans les Notarches, et l'opercule même se réduit pour quelques espèces à un foible rudiment de la membrane qui l'enveloppe, tandis que chez d'autres elle finit par disparoître entièrement.

On voit d'après cela qu'il est impossible de saisir le point juste où ces rudiments de coquille cessent d'être calcaires et où ils commencent à être uniquement membraneux, mais que si cependant on pouvoit trouver ce point, ce seroit vers la fin de la troisième section des Aplysies proprement dites, et non entre les Dolabelles et les Aplysies, ou pour mieux dire, entre la première et la seconde section.

Si nous comparons maintenant la forme de la coquille des Dolabelles et des Aplysies, nous verrons que ce n'est point encore là que l'on peut espérer de trouver des motifs de justifier la distinction que nous repoussons. Cette forme, considérée dans les caractères d'ensemble, est la même, mais on conçoit qu'elle doit être modifiée selon les sections et même les espèces que nous avons établies sur la connoissance plus essentielle des animaux. Ces coquilles sont oblongues, elles ont toutes une échancrure au côté droit postérieur, une crosse en arrière dans laquelle on distingue quelquefois un commencement de spire, et leur surface, plus ou moins disposée à l'enroulement, est toujours courbe; enfin elles ne sont que rudimentaires, mais à différents degrés, comme nous allons le voir, les moins incomplètes à la tête et les plus incomplètes à lá queue. En parcourant cette série, nous suivrons facilement encore la marche décroissante qui se montre tout aussi bien dans la forme de ces coquilles que dans leur nature. En effet les premières qui se présentent sont les Dolabelles (s.-G. Aplysies proprement dites, $\mathrm{I}^{\mathrm{re}}$ section): on distingue très-facilement un tour et demi de spire dans quelques-unes d'elles, leur surface recourbée obliquement annonce le mouvement d'enroulement de coquilles d'un ordre plus élevé; enfin une échancrure au côté droit lui donne à peu près la forme d'un triangle sphérique; celles qui viennent après (s.-G. Aplysies proprement dites, ${ }_{2}{ }^{\mathrm{e}}$ section) ont le sommet beaucoup moins apparent, et leur forme, à peu près quadrangulaire, n'offre plus autant, dans leur courbure, ce mouvement oblique d'enroulement si sensible dans la première section; l'échancrure est moins profonde, et dans quelques espèces les angles commençant à devenir plus obtus, annoncent une nouvelle modification dans la forme générale. Viennent enfin les coquilles de la troisième section (S.-G. Aplysies proprement dites, $3^{\mathrm{e}}$ section); leur sommet est encore moins spiral, surtout chez les dernières espèces; l'échancrure est réduite à peu de chose, et leur surface arrondie, peu courbe et toujours concave, n'offre plus aucun vestige d'enroulement. Si, après cette explication, on porte les yeux sur la suite des coquilles représentées dans nos planches, nous ne doutons pas que l'on ne reconnoisse, dans cette marche décroissante de la forme des coquilles de 
notre genre Aplysie, les modifications assez généralement suffisantes qu'éprouvent à la suite les unes des autres, les espèces que nous présentons. Il est permis de croire que de nouvelles découvertes peuvent venir remplir dans notre série, les lacunes un peu fortes que l'on y remarquera, comme plusieurs des nôtres l'ont déja fait. Maintenant noụs demanderons encore où est le point où s'arrête la forme des coquilles de Dolabelles et où commence celle des coquilles d'Aplysies? Certainement il n'est pas plus entre la première et la seconde section qu'entre la seconde et la troisième.

Ce n'est donc pas non plus dans la forme du test des Dolabelles qu'existe un caractère générique, et nous sommes en droit maintenant d'établir que ce Mollusque n'en possède point qui lui soit propre; nous ajouterons, pour rendre plus évidente son analogie avec l'Aplysie, qu'il répand comme elle une liqueur pourprée, abondante, et que ses moeurs, ses habitudes et son habitation sont, à très-peu de chose près, les mèmes.

Quant au genre Notarche de M. Cuvier, nous avons cru, par les mêmes raisons, devoir le réunir aux Aplysies, c'est-à-dire qu'il ne nous a pas offert de distinction générique assez importante. Cependant, sous le rapport de quelques caractéres extérieurs, ce Mollusque est encore plus analogue aux Aplysiès que les Dolabelles; car il a bien, il est vrai, les côtés du manteau serrés sur le dos, sans expansions natatoires, mais il n'a point d'aplatissęment postérieur. Du reste, les branchies sont semblables et situées de la même manière, les tentacules sont en nombre égal et de même forme ; toute l'organisation interne reconnue par M. Cuvier est la même que celle des Aplysies, et il ne se trouve qu'une seule différence assez ịportante, c'est le défaut d'opercule solidifié par un rudiment testacé.

Nous n'avons connu que récemment les individus qui ont servi à M. Cuvier pour l'établissement du genre Notarche; et ce savant, toujours empressé de seconder les efforts des naturalistes de ses lumières et des moyens dont il est entouré, a bien voulu les mettre à notre disposition; c'est sur ces individụs mêmes que nous avons pris les figures du Notarche de notre planche XXIII. Mais depuis long-temps déja nous connoissions des Mollusques tout-à-la-fois analogues aux Aplysies, et privés de coquilles, dont la fente dorsale, très-petite et dépourvue de lobes natatoires, se présentoit souvent dans un sens oblique; d'abord nos Aplysies citrina et Pleii, puis l'A. longicauda de MM. Quoy et Gaimard, enfin l'A. limacina de M. de Blainville; nous étant assuré que ces animaux ne différoient en rien de bien essentiel des. Aplysies à coquille, nous n’avions pas jugé nécessaire de les en séparer par une distinction générique. Nous avons en effet plusieurs sortes de Mollusques qui se montrent dans le même cas, les Pleurobranches entre autres présentent quelquefois un rudiment et quelquefois aussi en sont privés; les Limaces, les Sigarets, etc., en sont encore des exemples, Cependant désirant avoir égard non-seulement à ce caractère, mais à quelques autres encore moins importants, il est vrai, nous avions formé de ces espèces, réunies sous le nom d'Aclésies, un sous-genre du genre Aplysie, comme on peut le voir par les planches XX, XXI et XXII de notre Monographie. Nous avons donc fait la faute de méconnoître dans ces espèces et dans celles qui sont venues s'y joindre depuis, le genre Notarche de M. Cuvier, dont nous n'avions encore eu connoissance que par la courte description qui se trouve dans le second volume du Règne animal. Nous sommes assez heureux pour pouvoir à temps réparer cette erreur, en substituant à la dénomination d'Aclésie celle de Notarche imposée par ce savant bien long-temps avant que nous eussions rencontré des Aplysies sans rudiment testacé. Comme 
la connoissance du Notarchus gelatinosus ne change rien aux rapports qui existent entre les espèces privées de test et les Aplysies proprement dites, nous n'en continuerons pas moins à présenter ces premières comme sous-genre des Aplysies.

Nous avons cru devoir aussi restituer à ce genre l' $A$. viridis de $\mathbf{M}$. Bosc qui en a été retirée par M. de Férussac pour figurer dans les Actéons d'Ocken; l'examen de la figure de cette espèce, qui paroît se rapporter davantage aux Aplysies qu'aux Actéons, nous a décidé à ce changement; il est à désirer que de nouvelles informations viennent nous éclairer sur un Mollusque dont la description est si incomplète. Le genre Aplysie, tel que nous le présentons aujourd'hui, renferme donc les Aplysies de Linné, plus les Dolabelles et les Notarches. Nous allons examiner maintenant comment on peut faire usage des différences d'organisation qui ont servi à l'établissement de ces trois genres de Mollusques pour former des divisions secondaires dans celui que nous obtenons de leur réunion.

Classification. D’après ce que nous avons dit de la composition du genre Aplysie, on doit sentir la nécessité d'établir un mode de classification pour les espèces qu'il renferme actuellement. La méthode que nous avons adoptée pour atteindre ce but a l'avantage de conserver réunies celles qui, comme les Notarches et les Dolabelles, présentent une analogie de forme bien tranchée, et par conséquent de rendre plus facile leur détermination. Jue nombre considérable de ces espèces et la manière dont elles se lient les unes aux autres, dans cette méthode, nous donnent. lieu de croire que nous avons signalé les principaux groupes qui peuvent exister dans ce genre à la fois si riche et si intéressant.

M. Cuvier est le premier qui ait indiqué, comme nous avons déja eu occasion de le dire, un caractère propre à l'établissement d'une coupe dans les Aplysies. Ce caractère consiste dans l'absence ou la présence d'une ouverture à la membrane supérieure de l'opercule, et pouvoit servir à former une division dans le petit nombre d'espèces qu'il connoissoit alors. M. delle Chiaje est celui qui a cherché avec le plus de soin à établir une division convenable dans sa Monographie. Nous avons fait connoître l'heureuse découverte faite par ce savant sur l'opercule des Aplysies; elle lui a donné lieu de s'assurer que toutes les espèces étoient munies d'une ouverture à la membrane operculaire; mais que chez quelques-unes cette ouverture, au lieu d'être simple, comme celle que M. Cuvier a fait connoître dans l' $A$. punctata, étoit petite et située à l'extrémité d'un petit tube conique, souvent à peine apparent. Se fondant sur ce fait, il forme deux sections; la première comprend les espèces munies de ce tube, et la seconde celles qui ont une large ouverture. Si ce naturaliste s'étoit borné à appuyer sa division sur ces caractères, il auroit bien fait, car c'étoit un perfectionnement réel et qui pouvoit en quelque sorte suffire pour le peu d'espèces qu'il décrivoit; mais point du tout, il chercha à consolider l'établissement de ses sections par de nouveaux caractères, et il nous paroît alors s'être complètement égaré. Il pense que les espèces munies d'un tube, c'est-à-dire celles de la première section, ont une coquille membraneuse, et sécrètent seules la liqueur pourprée, tandis que les espèces qui montrent une ouverture large, c'est-à-dire celles de la seconde section, auroient une coquille calcaire et répandroient seules la liqueur âcre et venimeuse. Ces deux distinctions nous paroissent également fausses. D'abord, comme nous croyons l'avoir suffisamment prouvé en traitant de la réunion du genre Dolabelle au genre Aplysie, on ne peut diviser les coquilles de ces Mollusques en coquilles calcaires et coquilles membraneuses, puisque toutes sont à la fois plus ou moins calcaires et membraneuses. Dans tous les 
cas, nous avons sous les yeux les coquilles des $\mathcal{A}$. dactylomela, brasiliana, Sorex, Protea, etc., qui sont revêtues à l'intérieur d'une couche calcaire très-épaisse, et répondent cependant à la première section de $\mathbf{M}$. delle Chiaje, puisqu'elles ont un tube, et celles des $A$. longicornis et Ferussacii qui sont si peu formées qu'elles ressemblent à de simples pellicules membraneuses et répondent cependant à la $2_{2}^{\mathrm{e}}$ section de ce savant, puisqu'elles ont la membrane operculaire largement ouverte à sa partie centrale.

En second lieu, il n'est pas permis de croire que les espèces de la première section possèdent seules la liqueur pourprée, tandis que celles de la seconde seroient uniquement pourvues de la liqueur âcre et venimeuse; l'examen de plusieurs espèces à l'état de vie nous én fournit la preuve. Nous sommes certain, par exemple, que l'A. depilans répand les deux liqueurs, et il en est de même des $A$. dactylomela, Protea, etc. (I).

Nous ne pouvons donc point voir dans ces indications des caractères constants et qui marchent ensemble. Tout ce que nous pouvons emprunter à M. delle Chiaje pour la classification de nos espèces de la $3^{\mathrm{e}}$ section, puisque c'est à elle seule que répondent les siennes, c'est cette distinction qui repose sur la présence d'un tube ou d'une large ouverture à l'opercule, distinction qui est le complément de ce qui avoit été déja indiqué par M. Cuvier. Si ensuite quelques espèces se montrent plus abondamment pourvues de la liqueur pourprée, tandis que d'autres au contraire en répandent fort peu, mais en revanche sont plus copieusement pourvues de la liqueur blanche; si quelques espèces ont une coquille plus calcaire, tandis que d'autres en ont une plus membraneuse, nous nous servirons de ces particularités pour ajouter des caractères aux descriptions spécifiques. Ainsi donc le travail de.M. delle Chiaje ne nous fournit rien d'important sur le mode à suivre dans l'arrangement et la division des espèces, mais nous ne doutons pas que ce savant n'eût été beaucoup plus heureux, si , comme nous, il en avoit eu un plus grand nombre sous les yeux.

La Monographie de M. de Blainville, plus nombreuse que celle de M. delle Chiaje, devoit naturellement nous faire espérer une classification mieux entendue et plus naturelle. Nous avońs déja vu, en examinant le travail de ce savant, quel'on ne peut tirer aucun parti des caractères rarement constants et quelquefois de peu de valeur sur lesquels il établit ses deux sections; nous n'avons donc pas pu nous éclairer de ce qu'il a écrit sur les Aplysies, pour nous diriger dans le choix d'une classification à la fois commode, naturelle et durable. Nous allons développer celle que nous avons adoptée, afin de donner plus facilement lieu d'apprécier les motifs qui nous ont dirigé dans sa formation.

Le premier caractère qui se présente, et par conséquent le plus important, est certainement celui qui résulte de la présence ou de l'absence de la coquille dans l'opercule des branchies ; c'est celui qui a scrvi à M. Cuvier pour l'établissement du genre Notarche; quelques autres caractères moins importants viennent encore se joindre à celui-ci pour marquer d'une manière plus tranchée cette première division du genre; par exemple, le pied est généralement plus étroit dans les espèces dépourvues de test, l'ouverture bran-

(I) Le nom de Baril-de-vin, donné par les nègres pêcheurs de la Martinique à cette dernière espèce, prouve la vérité d'une partie de cette assertion, de même que celui de Pichevin (Pisse-vin), donné à l'A. depilans par les pêcheurs de l'embouchure de la Gironde. 
chiale est toujours très-petite et souvent oblique; ainsi donc nous partageons d'abord le genre Aplysie en deux sous-genres; le premier renferme les Aplysies proprement dites: il a pour principal caractère la présence d'un rudiment testacé, et le second comprend les Notarches et celles de nos nouvelles espèces qui s'y rapportent : il a pour caractère principal le défaut de test operculaire.

Cette première distinction établie, si nous examinons les espèces qui se rangent dans les Aplysies proprement dites, nous reconnaîtrons trois groupes ou sections bien tranchées, fondées sur des caractères d'un ordre secondaire.

$\mathrm{La} 1^{\mathrm{re}}$ section contient les espèces dont le corps, rétréci en avant et renflé en arrière, montre un disque terminal postérieur; les côtés du manteau sont étroits, serrés sur le dos et impropres à la natation; la coquille, de nature calcaire, est en forme de triangle sphérique. Cette section se compose des Dolabelles seules.

La $2^{e}$ section renferme les espèces dont le corps, rétréci aux deux extrémités, ne montre point de disque postérieur, et chez qui les lobes du manteau, toujours très-petits, sont impropres à la natation; la coquille, de nature plus calcaire que membraneuse, est de forme subquadrangulaire. L'A. clolabrifera, qui avoit été à tort introduite dans le genre Dolabelle, est le type de cette section dans laquelle plusieurs autres espèces nouvelles viennent se ranger.

La $3^{\mathrm{e}}$ section se compose de celles dont le corps, rétréci aux deux extrémités, a les lobes du manteau très-rilatés et propres à la natation. La coquille est membrano-calcaire et de forme subarrondie. Cette dernière section comprend le genre Aplysie de Linné, et c'est elle particulièrement qui montre à l'opercule ces caractères observés par MIM. Cuvier et delle Chiaje, et dont nous avons parlé plus haut; aussi nous la soumetirons à la division proposée par le second de ces naturalistes. Ainsi donc nous aurons deux groupes, l'un composé des espèces munies d'un petit tube conique à la membrane operculaire, et l'autre de celles qui, au lieu de cet organe, présentent une large ouverture.

Quant au sous-genre Notarche, nous n'avons point essayé d'y former de coupes secondaires, n'ayant point encore sous les yeux un assez grand nombre d'espèces à comparer.

Maintenant que notre classification est établie et que nos groupes sont placés dans l'ordre que nous avons jugé le plus convenable pour' suivre, à l'exemple de M. Cuvier, une marche descendante, nous allons porter les yeux sur toute la série de nos espèces, pour voir si en effet elles se lient suffisamment entre elles.

Les Dolabelles paroissent en tête de tontes les espèces, parce qu'elles présentent, dans la nature solide et moins rudimentaire de leur coquille et dans son développement évidemment spiral, un degré de perfectionnement très-remarquable. La section qu'elles forment se lie à celle qui la suit par la coquille d'une part, et par l'absence de lobes propres à la natation de l'autre; cette seconde se lie à la suivante par la forme générale du corps qui est rétréci aux deux extrémités, et par la forme et la nature de la coquille qui, dans les dernières espèces, commence à s'arrondir, comme dans la troisième section, et à devenir moins calcaire et plus membraneuse. Enfin cette troisième section se lie au sous-genre Notarche qui termine le genre, par l'ensemble des formes extérieures et surtout par le rapprochement des dernières espèces d'Aplysies proprement dites, dont le rudiment de coquille n'est plus, comme nous l'avons observé, qu'une mince pellicule, tandis que chez les Notarches ce rudiment disparoit complètement. 
On voit par ce court aperçu que la filiation de nos espèces se fait surtout remarquer dans l'opercule, et que notre série décroît insensiblement, depuis l'espèce dont la coquille est solide et bien formée jusqu’à celles où cette pièce disparoît.

La méthode que nous venons de faire connoîtrè est celle que nous avons dû présenter, parce qu'elle nous a paru la plus naturelle et la plus commode; nous n'avons pas la prétention de croire qu'elle puisse toujours suffire, mais nous espérons qu'elle sera de quelque utilité en ce moment, et nous serons bien récompensé de nos efforts, si, par la suite, on y trouvoit encore une base solide et durable pour la classification d'un genre si intéressant, et dont les nombreuses espèces embrassent presque toute la surface du globe.

Le tableau synoptique que nous joignons ici fera mieux concevoir ce que nous venons d'indiquer, en présentant à la fois sous les yeux l'ensemble de nos espèces et leur arrangement méthodique. 


\section{TABLEAU SYNOPTIQUE}

DU GENRE APLYSIE.

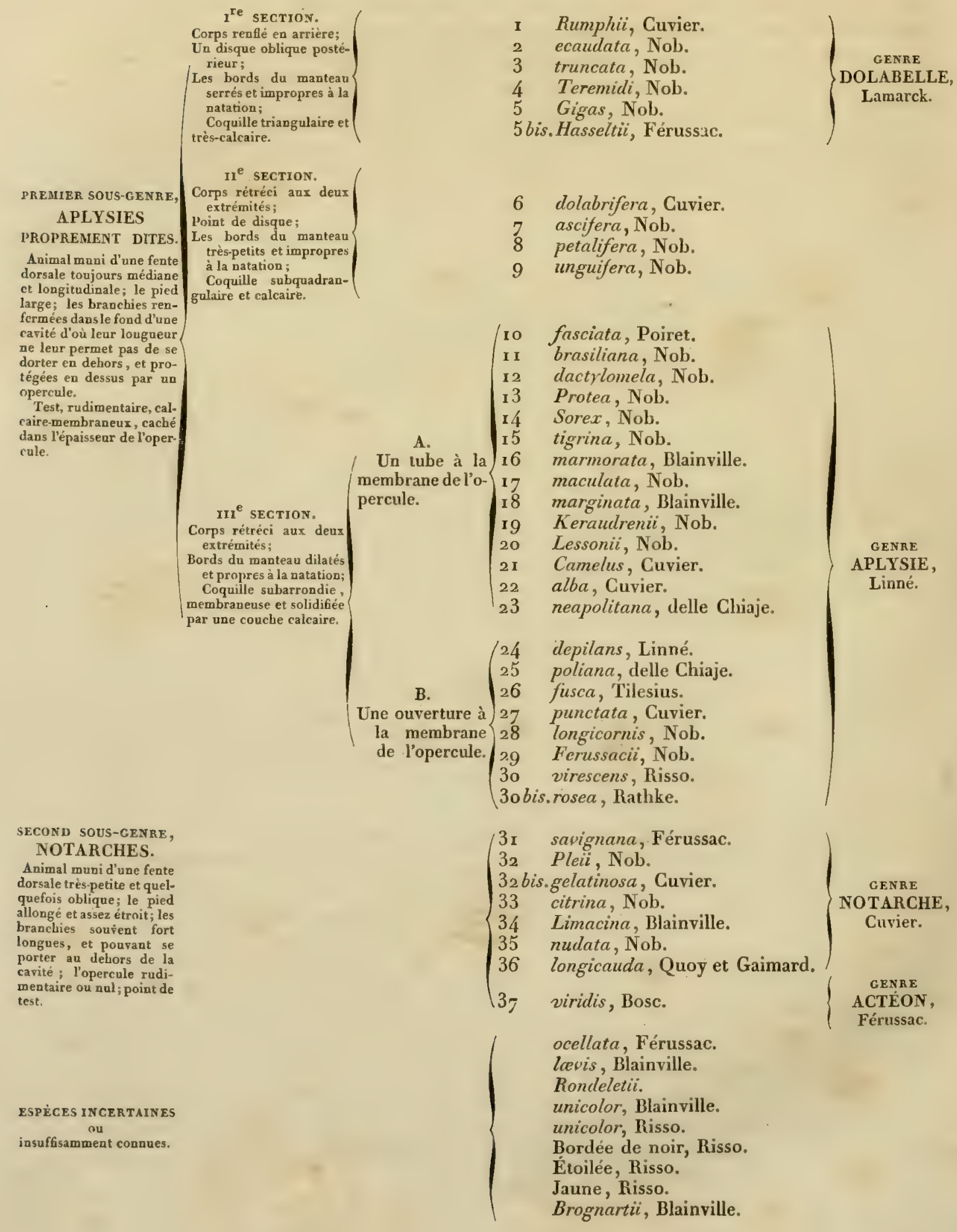




\section{premier sous-genre. APLYSIES PROPREMENT DITES, Nobis.}

\section{Lepus marinus, Rondelet, Gesner, Aldrovande, Fabius Columan; Lernea,}

Linné, Bohadsch; Tethys, Linné; Laplysia, Linné, Barbut, Bruguière, Bosc, Lamarck ; Aplysia, Gmelin, Cuvier, Roissy , Férussac, delle Chinje, Blainville; Dolabella, Lamarck, Guvier, Férussac, Blainville.

Animal muni d'une fente dorsale toujours médiane et longitudinale; le pied large; les branchies renfermées dans le fond d'une cavité d'où leur longueur ne leur permet pas de se porter au dehors, et protégées en dessus par un opercule.

Test, rudimentaire, calcaire-membraneux et caché dans l'épaisseur de l'opercule.

\section{PREMIÈRE SECTION.}

Limax marina tertia et operculum callorum, RUMPHIUs; Doris verucosa, Grielin, Barbut; Dolabella, Lamarck, Cuvier, Roissy, Férussac, Blainville, Sowerby.

Corps renflé en arrière; un disque oblique postérieur; les bords du manteau serrés et impropres à la natation.

Coquille triangulaire et très-calcaire.

Le corps des espèces qui composent cette première section est rétréci en avant, et trèslarge en arrière où il est toujours tronqué par un plan ou disque oblique; la fente dorsale est médiane et formée par le rapprochement des deux côtés du manteau qui sont trèsétroits et dont les bords sont serrés l'un contre l'autre, excepté cependant à l'extrémité postérieure où ils s'écartent un peu pour donner passage au siphon. Ces bords ainsi conformés sont impropres à la natation, n'ayant pas la moindre ampleur et ne pouvant que faiblement s'écarter l'un de l'autre. Le canal qui réunit l'orifice des oufs avec celui de l'organe mâle, est médian à sa sortie de la cavité dorsale; il se porte ensuite au côté droit. On remarque assez généralement que les tentacules postérieurs sont un peu plus rapprochés à leur base; le pied est grand.

La coquille, qui est toujours beaucoup plus calcaire, plus solide et plus formée que celle des autres sections, affecte une forme particulière qui est à peu près celle d'un triangle sphérique. Sa crosse ou sommet occupe l'angle postérieur; il est uniquement calcaire et toujours plus grand et plus épais que dans les autres Aplysies. Il forme au côté droit avec le bord de la coquille une échancrure anguleuse plus ou moins profonde. La face supérieure qui est marginée à gauche et en arrière par une sorte de duplicature du rebord, est toujours revêtue d'un épiderme épais et jaunâtre qui est le premier vestige de la partie membraneuse que nous verrons se développer davantage dans les autres sections, et la face inférieure, de même que toute la crosse, est très - blanche et d'un poli d'émail. Cette coquille, d'un prix encore assez élevé, est recherchée dans les collections. 
Les espèces de cette section répandent une liqueur pourprée très-abondante; c'est par ce moyen qu'elles parviennent à se dérober aux poursuites de leurs ennemis. Elles ne sont point organisées pour nager, mais en revanche leur pied qui est grand leur donne toute la facilité possible pour ramper. On les trouve sur les rivages dans les lieux tranquilles, tels que les ports et les baies; souvent elles habitent sur des fonds vaseux dans lesquels elles restent long-temps enfoncées, leur siphon s'élevant seul pour recevoir l'eau nécessaire aux branchies.

Elles sont très-répandues dans les mers de l'Inde, de l'Océanie et jusqu'aux Iles de la Société; et nous croyons pouvoir assurer que l'Europe et l'Amérique n'en possèdent point.

\section{ES PÈCES.}

1. APL̇YSIA RUMPHII, Gưrer. Pl. 1.

Limax marina tertia, et tertia species operculi callorum, Rширнго, Thes: Am. Pl. x, fig. 6 , et pl. $x_{\mathbf{L}}$, fig. $\times$.

Doris verucosa, Gmexir, Syst. nat., p. 3 ro3. BАввот, the Genera vermium. Pl. Iv, fig. I.

Dolabella callosa, Lamarck, Syst. des an. sans vert. Prem. édit., fig. 62 .

Dolabella Rumphii, Cuvier, Ann.duMuséum, V, p. 437 , Pl. xxrx, 1, et Riegne an., t. II, p. 398 .

Dolabelle calleuse, Dolabella Rumphii, LAMarcK, An. sans vert. Seconde édit., t. VI, $2^{e}$ partie, p. $4 \mathrm{r}$.

Dolabella Peronii et Dolabella Rumphii, BuAsNville, Dict. des sciences nat. et Manuel de malacologie., p. 473.

Dolabella Rumphii, Férussac, Tabl. syst.; p. xxx.

Dolabella Peronii et Dolabella Rumphii, DesHAYES, Dict. class. d'hist. nat., au mot DoLABELLE。

Corpore scabro, hirsuto, obscuro-virescente; disco fimbriato.

Testâ dilatatâ, suprù luteâ, subtùs albâ ; rostro subspirali, crasso, calloso.

Animal. Son corps est couvert de petites aspérités aiguës, quelquefois très-allongées, et les bords du disque sont comme frangés ; les côtés du manteau sont extrêmement serrés sur le dos; le pied est très-grand ; la peau est épaisse, rude, et rélléchit une couleur verdâtre assez vive. Longueur $0,382$.

Coquille. Elle est large et porte une échancrure profonde au côté droit postérieur. Sa surface supérieure est revêtue d'un épiderme jaune fort agréa- ble, et sa surface inférieure est blanche et émaillée; le sommet est subspiral, épais, calleux, large et irrégulier. Longueur o,o64.

Description. L'Aplysie de Rumphius est très. grande, elle atteint jusqu'à 12 et 14 pouces de longueur; sa partie postérieure est très-renflée, tandis que l'antérieure se rétrécit insensiblement; son disque est large, très-aplati, et serait parfaitement circulaire sans les contractions continuelles $\mathrm{dn}$ mollusque, qui en font, sans cesse, varier la forme; l'extrémité inférieure de ce disque laisse quelquefois apercevoir un commencement de queue, mais souvent cette partie est à peine plus saillante. Toute la surface du corps, de même que celle du disque, est hérissée de petites expansions aiguës, plus ou moins allongées, et qui, lorsque l'animal est dans l'eau, se dressent; mais elles s'affaissent et se couchent sur la surface du corps dès qu'il est hors de son élément. Les bords du disque, qui en sont plus munis, paroissent comme frangés. Les lobes du manteau sont extrêmement serrés, la fente dorsale est courte, et ses bords sont peu susceptibles d'écartement. Cette fente se prolonge en arrière jusquau centre du disque, où elle s'élargit en formant une ouverture presque circulaire pour le passage du siphon. En avant elle se termine presque à la moitié de la distance qui sépare le bord du disque de la base des tentacules postérieurs. Le pied est grand et calleux; il est quelquefois trèsdistinct du corps, et quelquefois aussi on le confond facilement avec lui; cela dépend des contractions plus ou moins grandes de cette partie du mollusque. Les tentacules n'offrent rien de particulier.

La couleur, qui est généralement verdàtre obscur, varie en vivacité, et quand l'animal est vivant et dans l'eau, elle réfléchit parfois un éclat métallique. 
La coquille est grande, assez bombée, large, profondément et largement échancrée; son bord postérieur et son bord gauche sont bordés par un repli régulier très-marqué; son bord antérieur est mince et membraneux. La face supérieure, à l'exception de la crosse, est recouverte d'un épiderme d'une belle couleur jaune, mais que le frottement enlève facilement; la face inférieure est blanche, toute calcaire et émaillée; on y remarque plusieurs stries longitudinales et transversales assez visibles, et une côte très-allongée prolongeant le bord gauche qu'elle avoisine. La crosse est épaisse, calleuse, irrégulière, large, sans épiderme, blanche et émaillée; elle présente une disposition spirale assez sensible.

Cette espèce, qui est le type de la section, est la première connue; c'est sur sa coquille que II. de Lamarck avoit formé le genre Dolabelle, et sur son animal, rapporté des mers de la NouvelleHollande par Péron, que MI. Cuvier a fait ses recherches anatomiques.

Habitations. L'Aplysie de Rumphius paroît répandue dans toutes les mers de l'Asie. Rumphius la cite aux Moluques; Péron l'a rapportée de Timor; MIM. Quoy et Gaimard de Waigiou et de Rawack, et nous de l'lle de France: elle vit dans les lieux tranquilles sur le sable et la vase. Nous ne nous sommes point aperçu qu' elle vint, comme certaines Aplysies, respirer l'air libre sur le bord du rivage.

Cabinet d'Anatomié du Jardin des Plantes, AprySIE, $\mathrm{n}^{\circ} \mathrm{I}$.

\section{A. ECAUDATA, Nobis. Pl. II.}

Corpore ecaudato, sublevigato, virescente; disci margine undulato; tentaculorum posteriorum basibus proximantibus.

Tèstâ translucidâ, suprà pallido-luteâ, subtùs albâ; rostro subspirali, crasso, subtùs calloso.

Animal. Moins grand que l'espèce précédente, et n'offrant ancune apparence de queue; la surface du corps n'est point hérissée, et montre seulement en avant et en dessus quelques tubercules aplatis; le bord du disque n'est point frangé, mais seulement irrégulièrement ondulé; les tentacules postérieurs sont très-rapprochés à leur base; la couleưr est verdâtre. Longueur $0, \mathbf{r} 35$.

Coquille. Elle est semblable à la précédente; son sommet est en dessous plus épais et calleux; l'épiderme moins épais. Longueur 0,028.
Description. Cette espèce est bien moins grande que la précédente, avec laquelle elle a de grands rapports; elle s'en distingue cependant suffisamment par l'absence de frange au pourtour du disque et d'aspérités sur toute la surface du corps. Son disque est uni, moins grand à proportion que celui de l'A. Rumphii, et montre encore moins d'extrémité caudale; la fente dorsale est semblable, et les lobes sont également serrés; les tentacules postérieurs offrent un caractère qui peut servir à la faire reconnoître: c'est, que leurs bases sont beaucoup plus rapprochées que dans les autres espèces; la partie antérieure du corps montre quelques tubercules peu saillants et quelquefois des taches rembrunies; le manteau, qui est moins épais, moins rude, est de couleur verdâtre à peu près comme la précédente.

La coquille ressemble beaucoup à celle de l' $A$. Rumphii,; on pourroit la prendre pour un jeune individu de celle-ci; cependant le côté inférieur de son sommet est beaucoup plus calleux; sa partie antérieure est translucide, et son épiderme trèsmince et d'un jaune pâle.

Habitation. Waigiou et Rawack, d'où elle a été rapportée par MM. Quoy et Gaimard.

Cabinet d'Anatomie du Jardin des Plantes, ApLYSIE, $\mathrm{n}^{\circ} 2$.

\section{TRUNCATA, Nobis.}

Corpore subecaudato, pallido, verriculis obtusis cooperto; tentaculorum posteriorum basibus leviter proximantibus.

Testâ vitreâ, albâ; rostro spirali, crasso.

Animal. Il ne diffère en rien pour la forme de l'espèce précédente; le corps est tout couvert de tubercules obtus; les tentacules postérieurs sont rapprochés, mais moins que dans l' $A$. ecaudata; sa couleur est pâle. Longueur o, 105 .

Coquille. Elle est vitrée; son sommet est épais, sans callosités, et montre inférieurement un tour et demi de spire bien marqué. Longueur o,or 8 .

Description. L'individu sur lequel nous avons établi cette espèce étoit dans un état qui ne nous a point permis d'en prendre une description suffisante, non plus que d'en tirer une figure; aussi nous ne lui affectons une distinction spécifique qu'avec doute, et seulement pour fixer à son sujet l'attention des naturalistes qui parcourront les pays d'où elle a été rapportée. 
Habitation. Waigiou et Rawack, d'où elle a été également rapportée par MIM. Quoy et Gaimard.

Cabinet d'Anatomie du Jardin des Plantes, AprX$\operatorname{SIE}, n^{\circ} 3$.

\section{A. TEREMIDI, Nobis. PI. III, f. 1-3.}

Corpore subecaudato, scabro, hirsuto, virescente, annulis albis nigrescentibus in medio; disci margine fimbriato.

Testâ elongatâ, supernè fusco-luteâ, subtìs albâ; rostro spirali, margine crasso.

Animal. Son manteau est très-épais et très-lur, tacheté de noir et de fauve avec des cercles blancs sur un fond verdâtre et hérissé de quelques aspérités aiguës; le bord du disque est un peu frangé. Longueur $0,13 \mathbf{I}$.

Coquille. Semblable à celle de l'A. Rumphii, mais plus longue et plus étroite; son épiderme est d'un jaune plus brun et est plus épais. Longueur $0,05 x$.

Description. Cette jolie espèce est très - grosse en arrière et rétrécie en avant; elle est à peu près de la taille des deux dernières, et s'en distingue facilement par les bords frangés de son disque; ses tentacules antérieurs paroissent un peu plus épais que dans les autres espèces; les côtés du manteau, très-étroits; semblent pouvoir s'écarter plus facilement l'un de l'autre, ce qui fait que l'opercule peut être mis plus facilement à découvert; les branchies sont roses, et la membrane qui recouvre l'opercule est bleuâtre; le siphon est assez long,

Toute la surface du corps est hérissée d'aspérités médiocrement allongées : elle est tachée de noir, de blanc et dé fauve sur un fond verdâtre; les taches blanches ont plus ou moins complétement la forme d'un cercle; le pied est large.

Cette espèce est très-distincte des autres, nonseulement par l'animal, mais encore par la coquille qu'il porte, et qui, avec les mêmes caractères que ceux de l' $A$. Rumphii, se montre cependant plus allongée.

Habitation. Elle vient des Iles de la Société, d'où elle a été rapportée par MM. Lesson et Garnot. Elle est très-commune sur les rivages de Taiti, Borabora et à l'île d'Oualan dans l'archipel des Carolines. C'est surtout sur lîlot de Tubaï, dans la baie de Borabora, que ces naturalistes l'ont observée en plus grand nombre. Les habitants la désignent sous le nom de Térémidi que nous lui avons conservé; mais ce qui la rend intéressante à nos yeux, c'est qu'elle est la première espèce connue qui serve de nourriture aux peuples près desquels elle ha. bite; c'est à MI. Lesson que nous devons la connoissance de cette particularité remarquable, dont nous avons déja parlé en traitant de l'utilité des Aplysies.

Cabinet d'Anatomie du Jardin des Plantes, ApLY* $\operatorname{SIE}, n^{\circ} 4$.

5. A. GIGAS, Nobis. Pl. III, f. 4 .

Corpore ?....

Testâ albâ, oblongâ, crassâ, supernè fusco-luteâ; rostro spirali, valde dilatato, subtùs infundibuliformi, striisque longitudinalibus exiguis notato; apice callosissimo.

Animal. Inconnu.

Coquille. Flle est très-grande, concave, épaisse, éminemment calcaire, portant une échancrure très-profonde; la crosse très-grande, en forme d'entonnoir inçomplet; la spire formant un töur et demi à deux tours assez distincts et terminée en dedans par un bouton saillant et irrégulier; un ou deux sillons longitudinaux et très-marqués près $\mathrm{du}$ bord gauche; épiderme épais. Longueur 0,102,

Description. Ce mollusque, que nous ne connoissons encore que par sa coquille, doit être d'une très-grande taille, si on en juge par celle-ci ; malheureusement nous n'avons aucun renseignement à son sujet. La coquille se distingue facilement des précédentes par sa crosse, qui n'est point, comme dans les autres, épaisse et calleuse; elle est, au contraire, assez mince, excepté au bord de léchancrure; elle est très-dilatée, et forme par sa courbure la moitié d'un entonnoir; sa surface intéricure est striée longitudinalemant, et sa spire, bien marquée en dessous, y forme plus d'un tour et demi bien visibles. Le sommet de cette spire, toujours du côté inférieur, est caché sous un bouton ou renflement arrondi très-saillant, mais irrégulier; cette coquille, fort épaisse, surtout près de son bord gauche, est munie dans cette partie d'un ou de deux sillons longitudinaux très-prononcés; une suture que l'on voit à la face inférieure de la crosse nous fait penser, ainsi que les nombreuses fractures qui se remarquent au côté gauche de l'individu que nous avons dessiné, que la coquille, à son état parfait, étoit munie, dans cette partie, d'un élargissement, dont nous avons figuré la forme supposée par une ligne ponctuée. Cette partie se- 
roit évidemment un des accroissements de la coquille, la crosse se trouvant le dernier.

La surface supérieure est revêtue d'un épiderme assez mince; la face inférieure est marquée de sillons longitudinaux et transverses.

Cette coquille n'est pas extrêmement rare dans les collections; cependant elle l'est plus que celle de l'A. Rumphii. En la comparant à celles des espèces précédentes, on peut croire que l'A. Gigas atteint $\mathbf{r} 6$ à $\mathbf{r} 8$ pouces de longueur, et peut-être davantage.

Habitation. La mer des Indes.

Collection de l'école royale des mines et celle du duc de Rivoli.

5 bis. A. HASSELTII, FéRussac. Pl. XXIV, f. I.

Dolabella Rumphii, van Hasseur, Lettre sur les Moll. de Java. Algem. Konst en letterbode, $1824, \mathrm{n}^{\circ} 2,3,4$.

Corpore ecaudato, scabro, valde hirsuto, virescente, maculis fuscis nigrescentibus in medio: disci margine incequaliter fimbriato; rimá dorsi ad extremitates dilatatâ.

Testâ?

Animal. Corps très-renflé en arrière, sans appendice caudal; manteau très-rude, de couleur verdâtre, avec de grandes taches obscures et hérissé d'expansions charnues et ramifiées; bords du disque irrégulièrement frangés; fente dorsale très-serrée dans son milieu et également ouverte en avant comme en arrière. Longueur $0, \mathbf{1} 90$.

Coquille. Ineonnue.

Description. Les grands rapports qu'il est im-: possible de ne pas remarquer entre cette belle espèce et l' $A$. Teremidi, nous portent à croire que peut-être il conviendroit mieux de ne pas les distinguer. Les naturalistes qui les verront à l'état de vie, ou peut-être même ceux qui connoîtront la coquille de l'A. Hasseltii, pourront seuls décider cette question. En attendant voici ce que cette espèce offre de remarquable. Elle est une des plus grandes que nous connoissions; sa partie postérieure est très-renflée, et son disque, très-large et aplati, n'offre aucun vestige de queue; cette disposition lui donne la forme d'un pain de sucre dont la base seroit un peu oblique. Le pied, autant qu'il est permis d'en juger par le dessin que nous avons sous les yeux, est peu large; le manteau paroît rude, et est couvert d'un nombre considérable d'expansions charnues, allongées, aiguës, ramifiées et groupées d'une manière fort irrégulière; ces organes, d'après l'observation de M. van Hasselt, "s'allongent, se rétrécissent, sont trèssensibles et servent comme d'antennes". La fente dorsale est longue, serrée dans son milieu, mais s'ouvre largement aux deux extrémités, pour la circulation de l'eau nécessaire aux branchies; l'ouverture postérieure n'offre rien de particulier, c'est la même que l'on voit dans les autres espèces, et qui donne passage au siphon; mais celle du devant est remarquable en ce qu'elle est beaucoup plus sensible dans cette espèce que dans les précédentes, et que ses bords se rabattent de chaque côté, comme le feroient de petits lobes: c'est cette observation qui nous a engagé à placer l'A. Hasseltii à la finde notre première section, comme faisant le passage aux espèces où les côtés de la fente dorsale venant à s'élargir insensiblement, finissent par acquérir une ampleur qui les rend aptes à la natation. Les tentacules sont en forme de cornets enroulés et placés la pointe en bas; les yeux, représentés par deux petits points noirs, se distinguent difficilement sur les individus vivants, et sont situés comme dans les autres espèces. Ce mollusque est d'une coulcur verdàtre, interrompue par un nombre considérable de taches foncées très-irrégulières et rapprochées les unes des autres. Ces taches sont d'un brun-noir au milieu et entourées d'une teinte fauve; souvent elles sont ellesmêmes variées par de plus petites taches noires ou pâles.

M. van Hasselt, à qui nous devons la connoissance de cette belle espèce, n'a point fait connoître la coquille que renferme l'opercule des branchies; comme il se pourroit que ce futt celle que nous avons décrite sous le nom d'A. Gigas, et dont l'animal ne nous est point conru, nous engageons les naturalistes qui seront à même de vérifier ce fait, à y porter une attention particulière.

Habitation. Elle vient du rivage de Peperbaai, à l'île de Java; où M. van Hasselt prétend qu'elle est rare. C'est d'après un dessin qué ce savant a fait faire sur les lieux mêmes, et qui a été communiqué à M. de Férussac, avec une libéralité digne de tout éloge, par MM. Temminck et van Haan, que nous l'avons fait figurer. 


\title{
DEUXIE IIE SECT ION.
}

\author{
Dolabella, Cuvier, Férussac.
}

Corps rétréci aux deux extrémités; point de disque postérieur; les bords du manteau très-petits et impropres à la natation.

Coquille subquadrangulaire et calcaire.

Les espèces qui concourent à former cette seconde section se distinguent de celles que nous venons de décrire, surtout par l'absence du plan oblique qui termine la partie postérieure de leur corps; elles sont bombées dans le milieu, rétrécies vers chaque extrémité, et effilées en avant; l'ouverture dorsale, qui ressemble à une fente, est courte, peu ouverte, et paroît quelquefois uir peu à droite de la ligne médiane; ses bords, quoique plus dilatés que ceux de la première section, sont encore assez étroits et serrent fortement le dos, sur lequel ils peuvent en partie se croiser pendant le repos; mais ils sont encore impropres à la natation; le siphon est de médiocre longueur, et sort par l'extrémité postérieure de la fente dorsale, qui est toujours bâillante, même pendant le croisement des bords du manteau (I). La partie inférieure du corps se dilate tout autour, de manière à donner une plus grande étendue au pied, qui est en effet toujours très-vaste; les tentacules antérieurs ne diffèrent en rien de ceux des autres espèces, mais les postérieurs montrent quelquefois à leur sommet, au lieu d'une terminaison aiguë, une sorte de capsule oblique formée par un repli; cette partic est très-variable dans sa forme. Assez généralement le manteau se montre couvert d'aspérités, de tubercules ou de rugosités. Il est encore à remarquer que presque toujours l'orifice de la vulve est un peu plus en avant du bord antérieur de l'opercule que dans les autres espèces.

La coquille porte des caractères non moins distincts. Elle est calcaire, co̊mme celle de la première section, surtout les premières espèces; car les dernières se lient aux coquilles de la troisième section par une texture déja moins épaisse et moins calcaire; et en même temps par un épiderme plus membraneux et plus solide. Elle est toujours plus longue que large, et dans quelques espèces la crosse est épaisse et calleuse.

Nous avons vu que dans la première section le test des espèces qui la composent se montroit de forme triangulaire; dans celle-ci il n'est pas précisément quadrangulaire, mais il présente d'une manière plus ou moins parfaite, selon les espèces, une disposition à la forme quadrangulaire; ainsi, comme le test de la troisième section est arrondi, celui de la seconde établit naturellement le passage de la première à la troisième.

(x) Nons nous sommes assuré, dans quelques espèces seulement, que la membrane de l'opercule étoit munie à son centre d'un petit tube presque imperceptible analogue à celui que l'on remarque dans cette partie chez quelques espèces de la troisième section; nous ignorons encore si c'est ici un caractère constant, ou s'il ne forme pas, comme dans la sectiun que nous venons de citer, seulement un groupe. 
Les Aplysies de cette section sont destinées à ramper, jamais à nager; elles sont de petite taille, et vivent dans les baies tranquilles, à l'entrée des rivières et jusque dans des mares d'eau saumâtre voisines du rivage; elles se cachent sous une légère couche de vase et à l'abri de grosses pierres. Nous n'en connoissons encore que quatre espèces, bien distinctes et venant de différentes mers. La Dolabella dolabrifera de M. Cuvier en est le type.

\section{A. DOLABPIFERA, Cuvier. Pl. IV, f, т-6.}

Dolabella dolabrifera, Guvirr, Règne animal, tom. II, pag. 398. Férussá, Tab. syst., p. $\mathrm{xxx}$.

Corpore hirsuto, virescente, maculis nigris proesertim ad marginem notato.

Testâ albâ, subtranslucidâ, recurvâ, angustâ, in medio interdum crassâ; rostro valde calloso.

Animal allongé, très-effilé antérieurement; les tentacules grêles; la fente dorsale petite et les lobes très-serrés; le manteau est de couleur verdâtre, tacheté de noir, surtout à la base, et hérissé d'aspérités très-aiguës; le pied uni et fort large. Longueur 0,ogo.

Coquille très-étroite, allongée, courbe, trèscalcaire; l'épiderme excessivement mince, de forme quadrangulaire, à crosse distincte et un peu calleuse; de couleur blanche, émaillée. Longueur o,oog.

Description. Cette espèce, qui est le type de notre deuxième section, se distingue parfaitement de celles qui l'avoisinent, mais surtout de celles qui la précèdent; elle est très-effilée antérieurement quand elle prend toute son extension et se montre arrondie en arrière. La cavité branchiale est trèsvaste, quoique son ouverture paroisse assez petite; l'opercule s'y montre très-étroit, et les branchies sont de couleur jaune; lorsque ce mollusque est dans l'eau, le manteau paroìt tout hérissé d'épines; mais ces épines, qui ne sont que de petites expansions molles et aiguës, s'affaissent et se courbent sur la surface du corps lorsquil est hors de son élément, alors on ne voit plus que les petites aspérités sur lesquelles elles se dressoient. La base de cette espèce étant très-déprimée, son pied se trouve fort large. La couleur du manteau est un verdâtre sale très-irrégulièrement marqué de petites taches noirâtres qui se multiplient surtout vers le bas; le pied est de couleur livide. Quand cette espèce se contracte, la surface de son pied se courbe de telle manière que la partie antérieure touche presque la postérieure.

La coquille varie assez selon l'âge; cependant elle montre toujours, et plus peut-être que les autres espèces de cette section, une forme quadrangulaire. Elle est un peu recourbée dans le sens de sa longueur, surtout près de son sommet. Celui-ci, qui est tout-à-fait postérieur, est quelqueefois trèsépais et calleux; toute cette coquille, qui est d'une grande blancheur, est un peu transparente et trèssolide.

Nous avons figuré, pl. IV, fig. 5 et 6 , un exemplaire unique parmi le grand nombre que nous avons examinés, qui nous a offert assez de différence avec ceux-ri poưr nous engager à le représenter; l'animal ne différant en rien des autres individus de l' $A$. dolabrifera, nous ne considérons cette coquille que comme une monstruosité,

Habitation. L'A. dolabrifera, que nous avons observée en grande quantité à l'ile Bourbon, habite dans la vase, sous les pierres submergées; nous n'avons pu la rencontrer que dans de petites mares d'eau saumâtre voisines du rivage, où se trouvoient également d'autres mollusques, tels que deux espèces de Néritines et une espèce de Pintadine.

Cabinet d'Anatomie du Jardin des Plantes, AprySIE, $n^{\circ} 6$.

\section{A. ASCIFERA, Nobis. Pl. IV, f. 7-9.}

Corpore luteo-fuscato, verruculis rotundis cooperto.

Testâ albâ, recurvâ, angustâ, valdè crassâ, callosâ, rostro callosissimo.

Animal de même forme que le précédent, seulement plus arrondi sur le dos; ouverture dorsale très-petite; manteau jaune-brun parsemé de petits tubercules obtus. Longueur 0,085 .

Coquille plus anguleuse que la précédente, recourbée, étroite, à sommet très-calleux; émaillée, 
épaisse et calleuse surtout dans son milieu. Longueur 0,009 .

Description. L'A. ascifera ne diffère pour ainsi dire point par sa forme extérieure de l'A. dolabrifera, dont elle a la grandeur; on peut seulement remarquer que, tandis que le dos de cette dernière s'élève un peu en pointe, celui de la première s'arrondit; du reste, le cou, les tentacules et les larges expansions de sa base sont les mêmes. Les caractères qui nous ont servi à la distinguer comme espèce sont pris dans son manteau, qui est de couleur jaune-brun et parsemé de nombreuses aspérités obtuses semblables à de petites verrues. L'ouverture de la cavité branchiale est fort petite, et l'opercule se montre un peu plus large que dans l'espèce précédente.

La coquille se distingue également par des caractères suffisamment reconnoissables. Elle est également allongée, étroite et recourbée, mais plus élargie dans son milieu, plus anguleuse, et surtout beaucoup plus épaisse dans son centre, qui est à la fois calleux et émaillé; sa crosse est plus calleuse ct assez petite. Nous considérons cette coquille, toute proportion gardée, comme la plus calcaire de tout le genre. Elle est d'une blancheur parfaite.

Habitation. Elle a été rapportée de Saint-Jean de Cayenne par M. Richard, où nous pensons qu'elle habitoit dans la vase.

Cabinet d'Anatomie du Jardin des Plantes, AprySIE, $\mathrm{n}^{\circ} 7$.

\section{A. PETALIFERA, Nobis. Pl. V, for-3.}

Corpore leìi, virescente, aperturâ dorsi leviter dextratâ.

Testẩ albâ, concavâ, dilatatâ, exili.

Animal un peu plus petit que le précédent; man. teau uni et de couleur verdâtre; ouverture dorsale très-petite et un peu à droite de la ligne médiane. Longueur 0,0ว5.

Coquille mince, recouverte inférieurement d'un épiderme assez consistant, très-large et concave; la crosse très-petite et un peu recourbée vers l'échancrure, qui est plus distincte que dans les deux espèces précédentes. Longueur 0,009.

Description. Cette espèce offre encore la même forme extérieure que les précédentes; mais elle se distingue par sa belle couleur verte unie, et par une peau lisse et tellement mince que la blancheur de sa coquille se fait remarquer à travers sa trans- parence. Sa coquille commence à établir un rapprochement entre la seconde section et la troisième; car elle devient plus large, plus concave, et ses angles plus obtus lui donnent déja en partie la forme arrondie de celles de la troisième. Elle est beaucoup plus mince que le test des $A$. dolabrifera et ascifera, et montre un épiderme déja assez solide. Sa couleur est blanche.

Habitation. Elle vient des mers de Nice, d'où elle a été envoyée au Muséum d'histoire naturelle par. M. Risso.

Cabinet d'Anatomie du Jardin des Plantes, APLYSIE, $n^{\circ} 8$.

\section{A. UNGUIFERA, Nobis. PI. V, f. 4-7.}

Corpore virescente, obscuris nebulosisque maculis notato, verruculis rotundis cooperto.

Testâ albâ, concavâ, dilatatâ, exili, quasi rotundatâ.

Animal un peu plus grand que le précédent; le manteau de couleur verdâtre avec quelques nébulosités obscures, et parsemé de petites aspérités obtuses; le canal des organes de la génération très-profondément marqué. Longueur 0,075 .

Coquille moins calcaire, plus mince, presque arrondie; la crosse petite et sans callosités. Longueur $0,01 \mathbf{1}$.

Description. L'A. unguifera paroît très-voisine de la précédente, non-seulement par son aspect extérieur, mais encore par sa coquille, qui est à peu de chose près la même; cependant nous avons pensé qu'il convenoit d'en former une espèce distincte, parce que son manteau, outre quil est marqué de taches foncées sur un fond verdâtre, se montre aussi couvert de petites verrues, tandis que $l^{\prime} A$. petalifera est toute lisse, et unie dans sa couleur.

Sa coquille, assez semblable à la dernière qui a été décrite, présente encore plus de disposition à la forme arrondie des espèces de la troisième section qui vont suivre; cependant, en la considérant attentivement, on $\mathrm{y}$ distingue facilement des vestiges de la forme quadrangulaire que nous avons signalée comme étant un des principaux caractères de cette seconde section.

Habitation. La Méditerranée, d'où elle a été envoyée à Paris par M. Risso.

Cabinet d'Anatomie du Jardin dés Plantes, AplyS1E, $\mathbf{n}^{\circ} 9$. 


\section{TROISIËME SECTION.}

Lepus marinus des anciens, Rondelet, Gesner, Aldrovande; Lernea, Linné, Bohadsch; Tethis, Linné; Laplysia, Linné, Bruguière, Lamarck; Laplisia, Bosc; Aplysia, Gmelin, Cuvier, Férussac, Blainville, delle Chiaje, Risso.

Corps rétréci aux deux extrémités; bords du manteau dilatés et propres à la natation.

Coquille subarrondie, membraneuse et solidifiée par une couche calcaire.

La troisième section comprend tout le genre Aplysie tel qu'il étoit avant nous. Elle est jusqu'à présent la plus riche en espèces, et tout nous porte à croire, en effet, que les animaux qui la composent sont de tout le genre les plus répandus dans la mer. Ce sont les espèces que l'on rencontre si communément sur nos rivages, et dont l'une, connue des anciens, a donné lieu à toutes les fables que nous avons citées. Leur corps est généralement très-bombé, allongé en avant, et terminé postérieurement par une queue peu allongée et plus ou moins aiguë ou arrondie. Les tentacules antérieurs sont très-dilatés, et les postérieurs, qui sont con tigus, sont fendus longitudinalement à leur moitié supérieure. Le pied est toujours large, et le manteau est muni latéralement de deux expansions très-vastes qui se rabattent en se croisant sur la cavité branchiale pendant le repos, ou bien s'écartent l'une de l'autre pendant le mouvement, et par leur agitation servent à la natation. Ce dernier caractère et la faculté qui en est le résultat n'appartiennent qu'à cette troisième section. L'orifice des œufs étańt situé à l'entrée de la cavité branchiale du côté droit, et les lobes du manteau étant très-écartés en avant de la ligne médiane, il s'ensuit que la rainure qui réunit les organes de la génération ne suit point cette ligne, et s'en écarte toujours de plus en plus sur la droite pour aller se terminer sous le tentacule antérieur.

La coquille est généralement grande, de forme subarrondie, membraneuse et quelquefois même papyracée; sa surface est solidifiée à l'intérieur par une conche calcaire plus ou moins épaisse. Elle est bombée, et porte au côté droit une échancrure quelquefois assez profonde et toujours largement ouverte; le sommet de cette coquille est en partie calcaire et ne montre que confusément cette disposition remarquable dans les deux premières sections, qui indique un commencement de spire.

C'est surtout ici que l'on rencontre les espèces qui sont affectées d'une odeur désagréable en même temps qu'elles sont plus copieusement pourvues des liqueurs qui suintent de l'opercule et du manteau.

Les Aplysies de la troisième section habitent généralement les rochers; elles se cachent sous les pierres, dans les crevasses ou parmi les plantes marines. On les rencontre quelquefois à de grandes distances du rivage, nageant à quelques pieds au-dessous de la surface de la mer. Lors de la saison des amours, elles se réunissent en nombre considérable sur les rochers, où il est très-facile de les trouver à marée basse.

Nous établissons dans cette section une coupe qui facilitera la détermination de ses 
espèces. Cette coupe est basée sur la différence qui se manifeste à l'opercule par la présence d'un petit tube conique ou par celle d'une ouverture simple. Les détails dans lesquels nous sommes entré au sujet de ce caractère nous dispensent d'en parler de nouveau.

\section{A. Un tube à la membrane dé l'opercule.}

10. A. FASCIATA, Poiret. Pl. VI et VII.

Premier Lièvre marin? Rondelet, de Piscibus marinis. Geswer, Animalium in mari. de mollibus, Ardrovande, de An. exs., p. 81 .

A plysia fasciata, Porret, Voyage en Barbaric, t. II, p. 2. GMelin, Syst.nat., xiII, I, p. vi, p. 3 ro3, no 2 .

Laplisia fasciata, Bosc, Hist. nat. des vers. $2_{2}^{\mathrm{e} e ́ d i t ., ~ t . ~ I, ~ p . ~} 74$.

Aplysia fasciata, Cuvier, An.du Mus. 3, p. 295 , pl. II-IV; et Règne an., t. II, p. 398. DE Rorssy, Hist. nat. des Moll., t. V, p. 173.

Laplysia fasciata, Lммапск, An. sans vert., t. VI, $2^{\mathrm{e}}$ partie, p. 39 .

Aplysia fasciata, Fínussac, Tab. syst., p. xxx et Dict. class. d'Hist. nat., t. I, p. 476. delle Cimaje, Memorie sulla Hist. e notom. del regno di Napoli; et Giorn. med. Nap. d'Inspruck. Brainvilue, Journ. de Phys., 96 , janv. 1823, p. 285 ; et Dict. des sc. nat.

A. vulgaris, Buninville, Journ. de Phys., 96 , janv. 1823, p. 285 ; et Dict. des sc. nat., an mot LIEVRE MARIN.

Dolabella Lepus, Risso, Hist. nat. de l'Europe mérid., t. IV, p. 44, pl. 1, p. $x, 2$.

Aplysia fasciata, Parraudeau, Catal. des Moll. de l'íle de Corse, p. $9^{6}, \mathrm{n}^{\circ} \mathbf{r} 9^{\mathbf{r}}$.

Corpore valdè convexo, levigato, nigro-violaceo, maculis, minimis pallidis, rà̀ sparsis; membranis minio marginatis.

Testâ dilatatâ, membranaceâ, fragili, flavâ, concavâ; incisurá leviter arcuatâ; rostro recurvo, exiguo.

Animal. Soh corps est grand, très-bombé; le pied peu large; les lobes très-longs, le manteau lisse, d'un noiràtre laqueux uniforme avec quelques taches pâles très-rares. Les bords des lobes et ceux des tentacules sont de couleur de carmin très-vif. Longueur 0,220 à 0,250 .

Coquille fragile, mince, papyracée, très-peu cal- caire, arrondie, large, munie d'une échancrure très-ouverte, à sommet corné et peu épais.

Description. L'A plysie fasciée nous donne, comme plusieurs autres sortes de mollusques, un exemple fàcheux de l'insouciance que les naturalistes ont quelquefois mise à observer les animaux qui peuplent nos rivages. Cette espèce, extrêmement commune sur nos còtes de l'Ouest, et que l'on retrouve encore, mais en moins grande abondance, sur celles du Midi, n’a été connue et décrite que lorsque Poiret la découvrit en Barbarie.

L'Aplysie fasciée est la plus grande de la section à laquelle elle sert de type; elle est molle, lisse, d'une couleur très-foncée mêlée d'une teinte laqueuse; cette couleur se change par le frottement en violet et en bleuâtre. Sur un grand nombre d'individus on remarque de petites taches pâles rarement éparses, et qui ne semblent produites que par la présence de quelque peu d'air sous l'épiderme; souvent ces taches n'existent pas. Les hords des lobes natatoires et ceux des tentacules sont marqués d'une ligne étroite et bien tranchée de conleur vive de carmin. La face intérieure des lobes est moins foncée et un peu grisâtre. Les tentacules antérieurs sont très-larges lorsqu'ils prennent tout leur développement, et lcurs bords se montrent ondulés: les supérieurs sont assez longs, coniques et en forme d'oreille de liève. Les expansions latérales du nanteau sont très-longues et en même temps très-larges, ce qui donne à l'animal les moyens de nager avec une grande facilité; celle de gauche est quelquefois plus ample que celle de droite, cependant nous ne croyons pas que ce soit un caractère constant. L'opercule est très-grand, la membrane qui le revêt paroît fréquemment déchirée ( $\mathbf{I}$ ). Le siphon est allongé et conique. Les branchies, qui débordent un peu l'opercule, se montrent d'une couleur claire.

(1) C'est à tort que nous avons porté une petite oaverture à là membrane de l'opercule dans la figure an trait de la planche VI; ce caractère doit être remplacé par un petit tube. 
Le pied de l'animal est très-long, peu large, mais susceptible de varier considérablement ses formes.

La coquille est presque toujours ronde; elle est peu bombée, très-mince, fragile et toujours pleine de fissures; sa couche calcaire se réduisant presque toujours à très-peu de chose, elle paroît transparente. Sa couleur est blonde. Son échancrure est longue et peu profonde, et son sommet, qui est corné, est petit, informe, et se détache assez difficilement de la membrane de l'opercule. La surface de ce rudiment testacé est marquée de stries nombreuses, les unes rayonnant du sommet, les autres concentriques et indiquant les différents degrés d'accroissement. Le bord antérieur est très-mince et tout-à-fait membraneux.

Cette espèce répand plus abondamment que toutes celles que nous connoissons la liqueur du manteau et celle de l'opercule; cette dernière surtout se montre en telle quantité qu'un seul individu peut teindre en un instant trois à quatre seaux d'eau. L'Aplysie fasciée répand une odeur assez forte, mais moins cependant que celle de l' $\mathcal{A}$. depilans, arec laquelle elle a les plus grands rapports; cette odeur, qui devient désagréable quand elle est trop répandue, tient un peu de celle du musc.

Habilation. On voit souvent l'A. fasciata traverser à la nage les baies et les rades, et les pêcheurs de nos côtes de l'Ouest, qui la distinguent sous le nom de Chat de mer et de Picherin, la rencontrent fréquemment dans leurs filets. Elle nage le ventre en bas, et non en haut, comme on l'a avancé; pendant ce mouvement, les tentacules antérieurs se développent et paroissent prendre part à l'action générale. Dans la belle saison elle vient s'accoupler sur le rivage; c'est alors que l'on en rencontre des quantités considérables, surtout lorsque la mer est.basse; car alors elles restent à sec respirant l'air libre et se mettant à couvert sous les pierres et les plantes marines. Dans la saison des orages, elles se retirent dans de plus grandes profondeurs.

L'A. fasciata habite les côtes de France dans l'Ouest, et le Midi. Elle'se trouve encore sur les côtes de Barbarie, d'où nous avons dit que M. Poiret l'avoit le premier rapportée.

Cabinet d'Anatomie du Jardin des Plantes, ApLysIE, $\mathrm{n}^{\circ}$ xo.

Observation. Nous avons eu occasion dè rencontrer quelques coquilles d' $A$. fasciata assez remarquables par leur déformation. L'une de ces coquilles, pl. VIII, fig. 4, présente à sa face interne une cloison transversale très-solide et de même nature qu'elle. L'autre, représentée par les figures 5, 6 et 7 de la même planche, est encore plus extraordinaire en ce qu'elle nous présente à sa surface supérieure la même disposition que la coquille connue sous le nom d'Ombrelle, c'est-àdire que, privée de la crosse que l'on remarque dans les autres coquilles d'Aplysie, elle porte son sommet près du centre, et en forme de mamelon aigu; d'un autre côté, sa surface inférieure présente la disposition d'une Crépidule à cause d'une cloison qui s'y montre aussi, mais qui au lieu d'être verticale, comme dans la première que nous venons de citer, est horizontale, et occupe un tiers de la surface totale.

Nous considérons ces coquilles comme des anomalies très-rares, n'offrant aucun caractère, important, et dont on doive tenir compte.

1 I. BRASILIANA, Nobis: Pl. VIII, f. 1, $2,3$.

Corpore elevato, anticè elongato, posticè brevi, fusco-nigrescente.

Testâ oblongâ, obscurn-luteâ; incisurâ quasi nullä; rostro leviter formato.

Animal très-bombé, allongé antérieurement, un peu raccourci postérieurement, portant l'opercule très en arrière; le tube de l'opercule assez volumineux pour être très-distinct. 'Les lobes du manteau très-grands; de couleur brune-foncée. Longueur o, 1 ro à 0,130 .

Coquille oblongue, de couleur jaune-obscur; le sommet peu formé; l'échancrure presque nulle.

Description. Deux caractères asséz remarquables distinguent au premier abord l'A. Brasiliana des autres espèces : le premier, c'est que, assez allongée dans sa partie antérieure, elle se montre, au contraire, un peu courte dans sa partie postérieure; le second consiste dans la position de l'opercule, qui se trouve placé plus en arrière, et par conséquent obliquant vers la queue. A ces caractères nous ajouterons la couleur du manteau, qui est d'un brun-obscur, et la forme des tentacules postérieurs, qui sont parfaitement coniques, tandis que les autres sont très-élargis. Les lobes sont vastes et allongés, et l'opercule assez grand. La coquille se distingue également; elle est de forme oblongue et de couleur jaune-obscur; sa face inférieure est revêtue d'une couche calcaire 
assez épaisse; son sommet est peu formé, et l'échancrure est presque nulle.

Habitation. Sur les rochers des côtes du Brésil. Elle est très-commune dans la rade de Rio-Janéiro, d'où elle a été rapportée par MM. Quoy et Gaimard.

Cabinet d'Anatomie du Jardin des Plantes, ApLYSIE, $\mathbf{n}^{\circ} \mathbf{I}$.

\section{A. DACTYLOMELA, Nobis. Pl. IX.}

Corpore valdè gibboso, informi, rugoso, luteopallido, annulis nigris sparsis; margine membranarum violaceâ.

Testâ dilatatâ, succineá; incisurâ profundè arcuatâ; rostro recurvo, triangulari, crasso, calloso.

Animal très-bombé et allongé aux deux extrémités; le pied large et callcux; le manteau rugueux, de coulcur jaune-pâle avec de nombreuses taches noires en forme d'anneaux; les lobes bordés d'une belle couleur de laque. Longueur o, 1 jo.

Coquille grande, très-bombée, un peu diaphane, blonde à l'extérieur, légèrement émaillée à l'intérieur; à sommet très-prononcé; l'échancrure assez profonde. Longueur 0,042 .

Description. Cette espèce, qui est toujours trèsbombée, varie considérablement ses formes; son manteau est rugueux; ses tentacules antérieurs sont peu dilatés; mais ce qui la distingue surtout, c'est la couleur de son manteau; il est jaune-pâle, diversement nuancé, et plus ou moins couvert, selon les individus, d'anneaux noirs, irréguliers et de diverses grandeurs. La partie intérieure des lobes, de même que la surface de l'opercule, montre de grandes taches noires qui affectent différentes formes, tandis que le bord de ces mêmes lobes est teint de violet.

Sa coquille est agréablement colorée de jaune pâle à l'extérieur, et dans l'àge adulte de l'animal, elle se garnit à l'intérieur d'une couche calcaire ímaillée.

Habitation. Nous avons trouvé cette belle espèce sur les rochers de la rade de Saint-Yago de la Praya, aux îles du cap Verd, et, d'après ce que nous avons entendu dire, elle se trouveroit encore aux îles Canaries. Elle répand une liqueur violette presque aussi abondante que celle de l'A. fasciata.

Cabinet d'Anatomie du Jardin des Plantes, AprySIE, $\mathrm{n}^{0} \times 2$.
13. A. PROTFA, Nobis. Pl. X, 1, 2, 3 .

Corpore valdè elevato, luteo-virescente, annulis maculis nigris, viridibus, rubrisque variegatis; anticè elongato.

Testâ dilatatâ, concavâ, supernè succineâ, subtùs argentatâ; incisurâ profunde arcuatâ, rostro recurvo, triangulari, crasso, calloso.

Animal. Corps mollasse; extrêmement bombé antérieurement; les tentacules assez longs; manteau lisse, de couleur variable, mais où dominent le verd et le jaune, ainsi que de nombreuses taches annuliformes variées de noir, de rouge et de verd. Longueur 0,160 .

Coquille. Large, à sommet très-saillant et triangulaire; elle est assez solide; sa couche calcaire est nacrée à l'intérieur; l'échancrure est peu profonde, mais assez large ; son épiderme est jaune. Longueu 0,036 .

Description. Cette jolie espèce, très - commune aux Antilles, est bien distincte de celles qui l'avoisinent. Son dos est extrêmement bombé; ses lobes sont grands, et lui permettent de nager avec beaucoup de facilité; les rebords de ces lobes se montrent souvent ondulés; le cou est effilé, et la partie postérieure pointue; les tentacules sont grands; le manteau est mollasse, le pied grand et l'opercule assez étendu.

La couleur est très-changeante; dans l'eau elle paroît verdâtre; à l'air elle offre un autre aspect; sa couleur générale est alors le jaunâtre réfléchissant une teinte dorée, et dans toutes les circonstances elle montre un grand nombre d'anneaux noirs, variés de verd et de rouge; ces couleurs, ainsi que la forme et la disposition de ces taches varient beaucoup, mais elles sont d'un bel effet, surtout pendant que l'animal est vivant. La membrane de l'opercule, ainsi que l'intérieur des lobes, sont revêtus de grandes taches noires et irrégulières. Dans l'état de conservation le corps prend une couleur livide, mais les anneaux noirs conservent toujours leur empreinte.

La coquille de l' $A$. Protea est une des plus belles du genre; elle est à l'extérieur d'une belle couleur de paille, tandis qu'en dedans, où elle est revêtue d'une couche calcaire assez épaisse; elle se montre quelquefois très-nacrée. Elle est de forme concave et élargie; son sommet, très-saillant, présente une crosse triangulaire en partie calcaire; l'échancrure est longue et peu profonde. 
Habilation. L'A. Protea viênt des Antilles, où elle esı connue par les nègres pêcheurs sous le nom de baril de vin, à cause de la belle liqueur qu'elle répand; elle habite sur les rochers; nous l'arons fréquemment réncontrée, et en nombre considérable, sur ceux qui forment la base du fort Louis, dans la rade de Fort-Royal, à la Martinique. C'est surtout pendant les mois de mai, juin et juillet qu'il faut la cliercher. Elle se trouvoit déja dans la collection du Jardin des Plantes, où elle a été envoyée d'abord.par M. Richard, et depuis par M. Plée.

Cabinct d'Anatomie cu Jardin des Plantes, ApLySIE, $\mathrm{n}^{0} \times 3$.

\section{I'4. A. SOREX, Nobis. Pl. X, f. 4-8.}

Corpore valdè elevato, oblongo, brevi, scabro, obscuro-virescente, maculis nigris marmorato.

Testâ oblongâ, exili, supernè flavâ, subtìs albâ;; incisurä quasi posteriore; rostro recurvo, informi.

Animal court, oblong et très-bombé; manteau épais et un peu rude; pied large et calleux; lobes étroits; couleur verdâtre-foncée, marbréc de taches noires. Longueur 0,050 .

Coquille fauve, ovalc-oblongue, mince, et portant l'échancrure très en arrière. Longueur' 0,025.

Description. Cette espèce, qui cst de petite taille, est surtout remarquable par sa forme raccourcie ; son dos est extrêmement bombé, sa partie postérieure aiguë, et l'antérieure très-courte; les tentacules ne diffèrent en rien de ceux des espèces précédentes; le pied est très-large et écarri en avant; il est épais et rugueux; les lobes sont étroits et serrés sur le dos, qu'ils ne recouvrent pas coniplètement. L'opercule est assez grand et de forme oblongue. La couleur de sa robe la dis... tingue également des espèces voisines 'telle est en général verdâtre-obscure, et marbrée de taches noires assez nombreuses.

La coquille, qui est très-mince et peu concave, eșt ovale-oblongue; elle porte un caractère assez remarquable, c'est que l'échancrure, qui d'ailleurs est peu profonde, est située très en arrière. Sa couleur est fauve en dessus et blanche en dessous.

Habitation. Nous ignorons d'où vient l' $A$. Sorex; nous pensons cependant qu'elle habite les rivages de quelques îles de l'Océanie; elle a été recueillie, pendant le voyage de la Coquille, par M. Lesson.
Cabinet d'Anatomie du Jardin des Plantes, AprySIE, $n^{0} 14$.

\section{A. TIGRINA, Nobis. Pl. XI.}

Corpore valdè elevato, tantilliun brevi, levi, nigro virescente, maculis nigris marmorato, parvulisque albidis diversè glomeratis; membranis lateralibus dilatatis.

Testâ ovato - oblongâa, membranaceâ , posticè acutâ; luteo-lividla ; incisurâ leviter arcuatâ; rostro valdè exiguo.

Animal très-bombé, un peu court, pointu en arrière; manteau lisse de couleur noire-verdàtre, marbrée de taches obscures et d'un grand nombre d'autres de.couleur pâle, arrondies et diversement groupées; les lobes grands. Longueur 0,115 .

Coquille. Ovale-oblongue, peu cóncave, mince, fragile, aiguë en arrière, et de couleur jaune-pâle en dessus; l'échancrure peu arquée. Longueur o,o33.

Description. L'A. ligrina est une des plus jolies espèces que nous connoissons; sa taille est moyenne, son corps est extrêmement bombé; la partie artérieure peu allongée, et la postérieure courte, mais aiguë. Le pierl est large et oblong; les tentacules antérieurs sont peu larges; les postérieurs sont comme dans les autres espèces. Les lobjes latéraux sont larges et assez allongés, et doivent lui donner les moyens de nager avec une grande facilité; l'opercule est de forme oblongue; le petit tube conique, qui caractérise le premier groupe de la troisième section, est très-peu apparent, et le siphon paroît médiocrement allongé. La couleur générale est verdâtre-obscure, mais elle est variée par deux sortes de taches; la première se compose de marbrures d'une teinte très-foncée; elles se montrent sur toute la surface du corps, à l'intérieur des lobes et même sur l'opercule; mais dans cette dernière partie ces taches affectent une autre forme et présentent l'apparence d'un réseau noirâtre sur un fond assez clair. La seconde sorte se compose de taches pâles très-nombreuses, petites, arrondies et diversement groupées; elles n'existent que sur la surface extérieure et en avant de l'opercule.

La coquille, qui ne nous a offert qu'une légère couche calcaire, est oblongue, peu concave, pointue en arrière, où elle se termine par un sommet peu prononcé, et porte l'échancrure assez large 
mais peu profonde. Sa couleur à l'extérieur est un jaune-livide.

Habitation. Elle a été rapportée de l'île de France par MIM. Quoy et Gaimard, et nous l'avons également observée sur les rivages de cette île.

Cabinet d'Anatomie du Jardin des Plantes, Apry. SIE, $\mathrm{n}^{\circ} 15$.

I6. A. MARMORATA, Blanville. Pl. XII, f. 6-9. Journal de Phys., 96, janv. x 823, p. 286, pl.? f. 3 et 4 ; et Dict. des Sc.nat., au not Lìvre MARIN.

Corpore ovato, posticè acuto, levi, obscuro-virescente, maculis nigris marmorato; membranis lateralibus dilatatis; siphone elongato.

Testâ ovatâ, clongatâ, valdè concavâ, membranaceâ, luter-lividâ, incisurâ quasi posteriore, paululìmque arcuatâ.

Animal. Corps ovale, peu bombé, pointu en arrière; lobes latéraux assez grands; siphon un peu allongé ; pied ovale; couleur verdâtre-obscure, marbrée de noir. Longueur o,o6o.

Coquille mince, fragile, peu calcaire, ovale allongée, très-concave et de couleur jaune-livide; l'échancrure est très en arrière et peu arquée. Longueur $0,020$.

Description. Le corps de cette espèce est ovale, peu bombé, ou du moins beaucoup moins que celui des précédentes; le cou est peu allongé, et l'extrémité postérieure assez pointue; les lobes sont grands et très-propres à la natation; le siphon est conique et assez allongé; l'opercule est large. Les tentacules et le pied n'offrent rien de remarquable. Le manteau nous a paru lisse; cependant M. de Blainville prétend que, dans l'état frais, cette espèce se montre couverte de petits tubercules sphériques, qui disparoissent dans l'état de conservation. Nous avons pu comparer les individus que nous avons recueillis nous-même avec ceux qui appartiennent à la collection de M. Brongniart, et qui ont servi à M. de Blainville pour l'établissement de cette espèce, et nous pouvons affirmer que nos individus, qui étoient bien de la même espèce, ne montroient point de tubercules lorsque nous les prîmes vivants. Au surplus, nous avons remarqué, et l'on peut s'en convaincre par quelques-unes des espèces d'Aplysies qui sont dans la collection du Jardin des Plantes, que les individus qui avoient un manteau couvert de tuber- cules ou d'aspérités; le morıtrent encore, malgré le temps déja fort long durant lequel quelques-uns ont séjourné dans l'esprit-de-vin. La couleur du manteau est obscurément verdâtre, et montre beaucoup de marbrures foncées.

La coquille est presque membraneuse, du moins elle n'offre qu'une foible couche calcaire, qui se détache facilement daus l'état de conservation, au moyen de l'esprit-de-vin, et souvent pendant la vie même de l'animal. Sa forme est ovale-allongée et très-concave; son sommet est foiblement recourbé vers l'échancrure et à peine formé; celle-ci est assez en arrière et peu arquée; sa couleur est jaunâtre.

Habitation. Les côtes occidentales de France, où elle vit sur les rochers.

\section{I7. A. MACULATA, Nobis. Pl. XII, f. 6-9.}

Corpore oblongo, valdè elevato, marginato, posticè obtuso, anticè elongato, levi, fusco-olengino, pallidis maculis rarè sparsis; operculo rubro; siphone clongato.

Testâ nvatâ, valdè concavâ, membranaceâ, rufá; incisurä quasi posteriore, parvá; rostro recurvo, crasso.

Animal oblong, très-bombé, déprimé à sa base, allongé en avant et obtus en arrière. Manteau lisse de couleur brune-olivâtre avec quelques taches pâles; les lobes de grandeur moyenne, la cavité dorsale très-ouverte, l'opercule rougeâtre, le siphon allongé; et les branchies roses. Longueur o,ogo.

Coquille ovale, très-concave, membraneuse et très-peu calcaire; à échancrure petite et presque en arriêre; la crosse un peu recourbée et épaisse; couleur rousse sur les deux faces. Longueur 0,016 .

Description. La forme générale de l'A. maculata est à peu près celle des autres espèces; elle est très-bombée sur le dos et allongée aux deux extrémités, mais beaucoup plus en avant qu'en arrière, où, au lieu d'être aiguë, elle est arrondie. Lorsqu'elle se met en mouvement pour ramper, son pied prenant toute l'extension dont il est susceptible, il arrive que la partie inférieure du manteau se déprime tout autour, comme nous l'avons vu pour toutes les espèces de la deuxiène section. Les tentacules antérieurs n'offrent rien de particulier; les postérieurs sont pointus et un peu allongés; mais leur sommet varie facilement de 
forme; les yeux sont assez apparents. Les lobes du manteau ne sont pas très-grands; cependant nous ne doutons pas qu'ils puissent servir à la natation; lorsqu'ils s'écartent l'un de l'autre, l'ouverture dorsale est très-vaste, et l'intérieur de la cavité est d'une couleur rougeâtre, qui se manifeste plus particulièrement sur l'operculè. Celui-ci se montre à peu près en forme de croissant, à çause du siplion, qui est fort grand; les branchies sont roses. Le manteau est lisse et de couleur brun-olivâtre; on y remarque beaucoup de taches pâles.

La coquille est presque toute cornée; cependant sa face interne présente encore, par son brillant, quelques vestiges de la couche calcaire qui revêt les autres; elle est un peu transparente et de couleur rousse. Sa forme est ovale, très-concave, et un peu recourbée à son sommet, qui est assez épais ; l'échancrure est petite, et située presque en arrière.

Habitation. Nous l'avons trouvée parmi les fucus sur les rochers de Table-bay, au cap de BonneEspérance.

I8. A. MARGINATA, Buainvilue, Journal de phys., 96 , janv. 1823 , f. 285 , et pl. ? f. 5 ; et Dictionnaire des sc. naturelles, au mot Lik̀vR MARIN.

Corpore albido-luteo, maculis rotundis ocellatis, fusco-nigris, rarè sparsis; membranis elongatis, angustis, cum maculis quadratis, alternatim fuscis albidisque ad marginem.

Testâ?.....

Description. M. de Blainville décrit ainsi cette espèce, dont il a vu plusieurs individus dans la collection du collége des chirurgiens à Londres: "Corps ellipsoïde, du moins dans l'état de con"traction; les expansions latérales aussi longues "que dans l'espèce précédente (l'A. fasciata), "mais beaucoup plus étroites; couleur générale * d'un blanc-jaunâtre parsemé de quelques taches « rondes, rares, ocellées, d'un brun-noirâtre; le "bord supérieur des expansions orné d'une série i de taches carrées, régulières et alternativement "brunes et blanches."

Longueur 2 à 3 pouces.

Habitation ?.....
19. A. KERAUDRENII, Nobis. Pl. XIII.

Corpore oblongo, valdè elevato, obscuro-virescente, maculis magnis nigrisque notato; membranis dilatatis; tentaculis anterioribus ad marginem undulatis; operculo vasto, siphone dilatato, longitudinaliter aperto.

Testâ elongatâ, posticè angustâ; subtus albâ, supernè fusco-luteâ; incisurâ elongatâ, sed leviter arcuatâ; rostro crasso, recurvo.

Animal oblong, très - bombé, peu allongé antérieurement, pointu en arrière; les lobes grands, l'opercule vaste, le siphon allongé et ouvert longitudinalement; le manteau est lisse et de couleur brune-verdâtre, avec de grandes taches noires. Longueur o, $\mathbf{1} 5$ o.

Coquille ovale, allongée, très-rétrécie en arrière; l'échancrure longue et peu profonde; le sommet épais et-recourbé; de couleur brun-jaune en dessus et blanche en dessous. Longueur 0,048 .

Description. L'A. Keraudrenii est une de nos plus belles espèces; c'est également une des plus grandes. Elle est très-élevée, de forme oblongue et peu allongée en avant. Sa partie postérieure se termine en pointe; ses lobes sont trèsgrands et très-propres à servir de nageoires; ils se lient en arrière de l'opercule : celui-ci est vaste et de forme oblongue; le tube qui s'élève à son milieu est plus distinct que dans beaucoup d'autres espèces; le siphon, qui est formé d'un grand repli, est long et largement ouvert dans toute son étendue. Les tentacules antérieurs sont larges et ondulés sur leurs bords; les postérieurs n'offrent rien de particulier; ils sont coniques. Le manteau de cette belle espèce est lisse, et sa couleur est un verdàtre-obscur orné de grandes taches noires très-irrégulières et assez rapprochées les unes des autres. Le pied est large, oblong et calleux.

La coquille est remarquable par sa grandeur; elle est peu concave comparativement aux autres, et de forme ovale; elle est pointue et trèsrétrécie en arrière: son échancrure est latérale, longue et peu arquée; elle occupe à peu près la moitié de la longueur totale de la coquille. Le sommet est assez épais, recourbé et triangulaire. Sa face inférieure étant revêtue d'une conche calcaire assez épaisse, se montre blanche, mais sa face supérieure est de couleur jaune-fauve. Nous n'avons connu qu'un seul individu de cette espèce, celui qui appartient à la collection du Jardin des Plantes, 
c'est pourquoi nous n'indiquons pas comme caractère spécifique un pli que nous avons remarqué à la coquille; ce pli part du sommet, et va se terminer au bord antérieur; il présente une arète saillante à la face inférieure, et par conséquent un sillon à la face supérieure. Ce n'est peut-être qu'un accident, comme ceux que nous avons déja signalés dans la coquille de l' $A$. fasciata.

Habitation. Nous ignorons de quel pays elle vient; tout ce que nous savons, c'est qu'elle faisoit partie de la belle récolte faite par M. Lesson pendant le voyage de la corvette la Coquille.

Cabinet d'Anatomie du Jardin des Plantes, ApLYSIE, $\mathbf{n}^{\circ}$ Ig.

\section{A. LESSONII, Nobis. Pl. XIV.}

Corpore valde elevato, carnoso, levi, grisenroseo; pede leviter angusto; tentaculis posterioribus lanceolatis, lineâque nigrâ longitrorsum in medio notatis.

Testâ ovatâ, leviter concavâ, succineâ, posticè rcutâ; incisurâ quasi nullä, rostro parvo.

Animal très-bombé, charnu, peu allongé en avant, court et pointu en arrière; manteau lisse et de couleur grisàtre-rosée, avec de petites linéoles roussâtres; pied oblong; lobes natatoires assez vastes; tentacules antérieurs épais et peu susceptibles de développement; tentacules postérieurs lancéolés et marqués dans-toute leur longueur d'une ligne noire. Longueur o, 1 zo.

Coquille ovale, pointue en arrière, concave, à sommet peu formé; l'échancrure longue et peu arquée; la face inférieure blanche et revêtue d'une couche calcaire; la face supérieure couleur de succin: Longueur 0,034 .

Description. Cette jolie et grande espèce, que nous nous faisons un plaisir de dédier à notre ami M. Lesson, qui l'a encore rapportée de son voyage, est très-bombée dans toute son étendue; elle est oblongue; son cou paroit s'allonger moins que dans la plupart des autres espèces; en arrière elle est courte et terminée en pointe; son manteau est lisse et souvent très-boursouflé ; la couleur est grisâtre mêlée d'une teinte légèrement rosée ; on remarque encore qu'elle est ornée d'un grand nombre de petites linéoles rousses et très-fines qui font l'effet de petits vaisseaux que l'on apercevroit au travers de l'épiderme. Les lobes sont grands et réunis en arrière de l'opercule; celui-ci est oblong-allongé, et se termine postérieurement par un siphon peu grand, mais très-ouvert; les tentacules antérieurs sont de moyenne grandeur, épais et fort peu enroulés; les deux autres sont en forme de fer de lance, sans pour cela être aplatis, et leur longueur est marquée d'une ligne noire très-apparente qui semble en осcuper l'axe. Le pied est oblong et peu large.

La coquille ressemble beaucoup à celle de l'espèce précédente, mais elle est moins grande, toutes proportions gardées. Elle est ovale, pointue en arrière, peu concave, et son échancrure, qui n'occupe pas la moitié de la longueur totale, est cependant assez longue et peu arquée. Le sommet est à peine formé. La surface inférieure, revêtue d'une couche calcaire, qui, comme dans la plupart des espèces, se brise et tombe facilement, est blanche, tandis que la surface supérieure est de couleur de succin.

Habitation. Elle a été recueillie sur les rivages de Payta, au Pérou, où MI. Lesson dit qu'elle est très-commune.

Cabinet d'Anatomie du Jardin des Plantes, AplySIE, $\mathbf{n}^{\circ} 20$.

21. A. CAMELUS, Cuvier. Pl. XV, fig. I.

A. Camelus, Cuvier, Ann. du Mus., tom. II, p. $29^{5}$, pl. I, f. I. De Rorssy, Moll., t. V, p. I 7 I, $\mathrm{n}^{\circ} 2$, pl. LII, f. 8. LAMARcK, An. sans vert., t. VI, ${ }^{e}$ part., f. 40. De Férussac, Tableaux systématiques, pag. xxx, et Dict. class. d'hist: nat., t. I, p. 476. DE BlarnvILLe, Dict. des sc. naturelles, au mot LiÉvre marin. Delie Chinje, Memor. sulla hist. nat. e notom. delle Aplysie, p. jo, et Giorn. méd. nap. d'Inspruck.

Corpore oblongo, levi, nigro, anticè elongatissimo, posticè acuto; membranis clilatatis, siphone parum elongato.

Testâ?.....

Animal. Le corps oblong; le cou excessivement allongé; la partie postérieure pointue; les lobes vastes; le siphon peu allongé; couleur générale noire. Longueur 0,195.

Coquille?....

Description. C'est à M. Cuvier que nous devons la connoissance de cette espèce, qu'il n'a vue que dans l'état de conservation et décolorée; aussi la description quil en a donnée ne pouroit-elle 
suffire. Depuis, M. delle Chiaje a prétendu l'avoir reconnue à l'état de vie, dans les mers de Naples, et le peu de renseignements qu'il ajoute à ceux que nous possédions, nous engage dे la conserver comme espèce; cependant il est bien à regretter que ce savant n'en ait pas donné une figure ou au moins une description détaillée, et dans laquelle entrât celle de la coquille; il résulte de cette négligence que l' $A$. Camelus nous laisse encore beaucoup à désirer pour sa distinction comme espèce. Voici, au surplus, tout ce que nous savons à son sujet. Le corps est pointu en arrière, dit M. Guvier, et revêtu d'une peau lisse. M. delle Chiaje, qui a vu l'animal vivant, prétend qu'il est de couleur noire. Le cou est, au rapport de ces deux savants, excessivement allongé; enfín les lobes sont amples, le siphon peu aliongé, et le tube de l'opercule de moyenne longueur. Quant à la coquille, nous n'avons aucun détail à son sujet.

Nous n'avons póint vu l' $A$. Camelus, et nous n'avons pas même rencontré dans la belle collection du Muséum l'individu qui a pu servir aux observations de M. Cuvier. Nous engageons les naturalistes qui se livrent à la recherche des mollusques de fixer leur attention sur cette belle espèce, qui, selon toute apparence, habite nos rivages.

Halitation. Les mers de Naples.

22. A. ALBA, Cutrer. Pl. $\mathrm{XV}, \mathrm{f}, 2,3$.

A. Alba; Cuvier, Ann. du Mus., 3, p. 295 , pl. I, f. 6. De Rorssr, Hist. nat. des Moll., p. 17r, $\mathbf{n}^{0}$ 3. Fénussac, Tabl. syst., p. xxx; et Dict. class. dhist. nat., t. I, p. 476 , au mot Aplysie. De Buanvilue, Journ. de phys., 96 , janvier 1823 , p. 287 , et Dict. des sc. nat, au mot LIĖVRE MARIN.

A. Camelus (jeune individu), delie Chine, Memor. sulla hist. e notom. delle Aplysie, et Giorn. med. nap. d'Inspruck.

Corpore oblongo, levi, albido, postice brevi, membranis lateralibus angustis.

Testâ ?.....

Animal. Corps oblong, peu allongé en avant et court en arrière; le manteau lisse et blarichâtre; les lobes étroits. Longueur 0,074 .

Coquille?....

Description. L'A. alba a été proposée avec quelques doưtes par M. Cuvier, en même temps que l'A.Camelus; mais ce savant n'a pas jugé à propos d'en parler de nouveau dans son Règne animal, sans doute parce qu'il ne la jugeoit pas suffisamment déterminée. Nous avons retrouvé l'individu qui a servi à son établissement, au $\mathbf{M u}$ séum d'histoire naturelle, où il étoit parfaitement conservé. En l'examinant attentivement nous avons été porté à croire que c'étoit en effet une espèce distincte de celles que nous connoissons, mais dont les couleurs auroient été entièrement détruites par l'esprit-de-vin. Du reste, il nous a été impossible d'ajouter de nouveaux caractères à ceux que M. Cuvier nous a fait connoître : ainsi donc ce n'est qu'avec beaucoup de doutes que nous la présentons, mais seulement pour fixer à son sujet l'attention des naturnlistes.' Déja M. delle Chiaje a cherché à la reconnoître, et il pense que $l^{\prime} A$. alba est tout simplement un jeune individu de l' $A$. Camelus.

L'A. alba diffère surtout de la précédente par la brièveté de son cou; son corps est très-déprimé; elle est généralement aplatie et peu bombée à sa partie dorsale; les lobes sont étroits; sa couleur n'est pas connue.

Habitation?....

Cabinet d'Anatomie du Jardin des Plantes, AprySIE, $\mathrm{n}^{\circ} 22$.

\section{A. NEAPOLITANA, delle Cuinje. Pl. XV} bis, f. $\mathbf{1}$.

A. Neapolitana, Delde Cinusr, Giorn. med. nap. d'Inspruck; et Memorie sulla Hist. e notomia delle Aplysie.

Corpore oblongo, anticè elongato, posticè acuto; membranis valdè dilatatis; branchiis brevibus, muticis; siphone pralongo; caudâ tuberculo conico exornatâ, pede angusto; pallio oleagino, maculis argentatis auratisque notato.

Testâ P.....

Animal oblong; le cou allongé; l'extrémité postérieure pointue; les lobes très-grands; les branchies courtes; le siphon allongé; le pied long et étroit; un tubercule conique sur la queue. Le manteau olivâtre et marginé d'une légère couleur de chair; de nombreuses taches rondes argentées et dorées. Longueur 0,180 .

Coquille?....

Description. L'A. neapolitana; dont nous devons là découverte et une bonne description à M. delle Chiaje, offre des caractères qui la distinguent par- 
faitement de toutes les autres espèces voisines et en rendent la détermination assez facile. Nous ne pouvons micux faire que de traduire la description qu'il en donne. "Cette espèce, dit-il, a les tenta" cules antérieurs et postérieurs passablement longs, "larges et hérissés. Les yeux occupent la posi"tion ordinaire, sont entourés de rides, proémi" nents et blanchâtres. Le cou et les lobes ont "une ampleur qui surpasse celle de ces mêmes "parties dans les espèces connues. Ces lobes sont "à peine réunis à la partie postérieure, et leurs " rebords sont entiers dans quelques parties et un "peu dentelés dans d'autres. La membrane de "l'opercule seroit fermée en dessus, si elle n'avoit "dans son centre un petit canal (le tube de l'oper"cule) par lequel l'eau s'introduit. Le siphon est " très-long, et commence à l'anus; les branchies " sont courtes comme mutilées à leur partie posté" rieure et débordent peu l'opercule. Je n'ai point "d'observation à faire sur le trou de l'organe gé" nital et de la vulve, sous laquelle on remarque "l'ouverture des glandules que l'on a crues venimeuses dans l' $A$. fasciata.
"Son pied est long, étroit et rempli de bour" souflures. Il est, en avant, plus court que le cou, " et en arrière il se termine en pointe, à environ un " pouce au-delà du périmètre des lobes. Sur cette " partie postérieure et au milieu on voit un petit "tubercule conique.

"Le corps de cette espéce est olivàtre, bordé "dans tous ses contours d'une ligne couleur de " chair. On y remarque beaucoup de taches rondes " argentées et entremêlées d'autres taches d'un jaune" doré qui en rendent l'aspect très-vague. Ces ta" ches sont les mêmes sur le cou, les tentacules et "à l'entour des yeux. J'ai conservé cette Aplysie "dans l'eau de mer pendant environ vingt-quatre " heures, et, lorsqu'elle se remuoit, elle offroit, "par l'ensemble de ses couleurs, un beau spectacle " aux yeux de plusieurs savants qui l'observoient."

M. delle Chiaje n'ajoute malheureusement rien au sujet de la pièce testacée de l'opercule, qui fournit toujours quelques bons caractères spécifiques.

Habitation. Les eaux de Naples, où elle se trouve avec l' $A$. poliana.

\section{B. Une ouverture à la membrane de l'opercule.}

\section{A. DEPILANS, LrNmÉ. Pl. XVI et XVII.}

Lepus marinus, Nicandre, Droscoride, Pline, Ápulée, Éliev.

Lernea, Linné, $5^{\text {e }}$ et $6^{\text {e }}$ éditions. Bomanscr, de Quibusdam anim., cap. 1, tab. 1-4.

Tethys limacina, LINNÉ, Io édit., p. 653.

Laplysia depilans, Linné, I ${ }^{\circledR}{ }^{e}$ édit., 2 , p. 1082, $n^{\circ}$ I. Bаввтт, the Genera vermium, p. 3I, pl. 1II, f. 5 et 6 . Brvguìre, Encycl. meth., pl. Lxxxir et Lxxxiv, copiée de Bohäsch. Lamarck, Anim. sans vert., ${ }^{\text {re }}$ et $2^{\mathrm{e}}$ édit. Pennant, British Zoology, t. IV, p. 35, pl. $\mathrm{xxI}, \mathrm{f} .2 \mathrm{I}$.

Aplysia depilans, Gmeris, Syst.nat., p. 3 ro3, $n^{\circ}$. Cuvien, Tabl. élém. de l'hist, nat. des anim., p. 387, pl. Ix, f. 3; et Ann. du Mus., 3, p. 255 ; et Règne an., t. II, p. 398 . DE Rorssx, Hist. nat. des Moll., t. V, p. I 70. Férussac, Tab. syst., p. xxx; et Dict. class. d'Hist. nat., t. I, au mot ApLysie. Bualyville, Journ. de Phys., 96, janv. 1823, p. 286 , pl. ?, f, 6; et Dict. des sc. nat., au mot Liève manin; et Traité de Malacologie, p. 472. Parnaudeat, Catal. des Moll. de l'ïle de Corse, p. $9^{6}, \mathbf{n}^{\circ} \mathbf{1} 90$.

Laplisia depilans, Bosc, Hist. nat. des vers, t. I, p. 74, pl. II, f. 5 .

Aplysia leporina, dexte Chinse, Mem. sulla hist. e notorn. delle Aplysie, et giorn. med. nap. d'Inspruck.

Dolabella fragilis, LamarcK, An. sans vert. SEвA, Mus., pl. $x$, f. 8 et 9 .

Corpore valdè convexo, oblongo, anticè elongato, sublevigato, fusco-livido, maculis pallidis numerosisque sparsis; membranis lateralibus valdè dilatatis; operculo oblongo, ajserturá in medio rotundâ, maculisque radiantibus circum ornatâ.

Testẩ oblongâ, concavá, corneâ, crassâ, flavâ; incisurá brevi, rostro recurvo, calloso, margine si. nistrorsim dilatuto.

Animal. Le corps est très-bombé, oblong, allongé en avant et pointu en arrière; le pied est large; le manteau est élevé en arrière, et les lobes assez amples. Le siphon est large et peu allongé ; les tentacules antérieurs sont très-déprimés et 
ondulés sur leur bord; les tentacules postérieurs coniques et assez allongés. La couleur générale est brun-livide, avec de nombreuses taches pâles, grandes et très-irrégulières; l'ouverture de la membrane de l'opercule est entourée de taches noires plus petites et disposées en rayons. Longueur o, 190.

Coquille oblongue, concave, peu élargie dans son milieu; cornée épaisse, jaune; l'échancrure très-courte et bien en arrière; le sommet recourbé, calleux et dilaté au bord gauche. Longueur 0,042 .

Description. L'A. depilans, la plus anciennement connue de tout le genre, en est le type; c'est cette espèce célèbre sur laquelle les anciens ont inventé toutes les fables merveilleuses que nous avons racontées en traitant des propriétés des aplysies; nous ne reviendrons donc pas sur ce sujet, nous dirons seulement que, quoiqu'elle ait été décrite par beaucoup de naturalistes, et notamment par Boharlsch, elle laisse encore beaucoup à désirer pour sa description extérieure; mais que cependant les figures, si souvent copiées, que ce savant a mises à la suite de son beau travail, sont trèsbonnes, et nous auroient, en quelque sorte, dispensé d'en produire de nouvelles, si, ayant eu fréquemment sous les yeux des individus de cette espèce, nous n'avions jugé plus à propos de les représenter sous différents aspects, afin de ne plus laisser d'incertitude, s'il est possible, sur leur détermination.

L'A: depilans, une des plus grandes du genre, est très-bombée sur le dos, surtout quand elle se contracte, comme dans la fig. 2, pl. XVI. Elle est oblongue, susceptible de beaucoup d'allongement en avant, et pointue en arrière; son manteau est très-élevé tout autour, mais surtout en arrière, ce qui fait que l'extrémité postérieure de l'ouverture dorsale se trouve beaucoup au-dessus de la queue et très-près de la partie supérienre du dos; les lobes sont grands, liés et serrés en arrière de l'opercule; le siphon est peu grand, oblique et sort entre les deux lobes; le pied est large, oblong et marqué dans tonte sa longueur d'une grande quantité de petites stries très-fines; il est séparé du manteau par une ligne assez bien tranchée, à laquelle les contractions du mollusque font prendre toutes sortes de formes; le cou est assez long, et se dilate facilement près du bord du pied; les tentacules antérieurs sont larges, se déroulent en éventail pendant le mouvement, et montrent des bords un peu ondulés. Les tentacules postérieurs sont coniques, allongés et fendus à leur partie supérieure; les yeux, situés comme à l'ordinaire, c'est-à-dire en avant de ces tentacules postérieurs et un peu plus rapprochés de la ligne médiane, sont trèspetits et fort peu apparents; ils sont bleuâtres; l'opercule est de forme oblongue et assez vaste; la membrane qui le couvre porte une ouverture arron. die ou ovale qui caractérise le second groupe de la troisième section. La cavité branchiale est peu vaste, et le peigne des branchies déborde peu l'opercule.

Le manteau est lisse et de couleur brunâtre, quelquefois livide et mêlée d'une légère teinte de pourpre; mais il est plus ou moins tacheté, selon les individus; dans quelques-uns, et ce sont particulièrement les jeunes, le manteau est presque sans taches, ou du moins celles-ci sont si peu marquées qu'elles paroissent à peine; d'autres sont comme ponctuées de blanc, et enfin il en est chez qui ces taches, de couleur pâle, mais blanches dans leur milieu et extrêmement variées dans leurs formes, sont tellement grandes et nombreuses, quils en paroissent tout couverts et grisâtres. Il arrive fréquemment quà la base du manteau, tout près $\mathrm{du}$ bord du pied, ces taches affectent une forme allongée et une disposition à peu près parallèle et verticale.

D'après ce que nous venons de dire de la conleur de l'A. depilans, on conçit que l'on pourroit distinguer plusieurs variétés, et nous pensons que ces variétés, si l'on n'y fait pas une attention scrupuleuse, peuvent induire en de graves erreurs, en faisant passer pour espèces distinctes des individus qui ne différeroient tout au plus que par un peu plus ou un peu moins de taches, par leur forme et même quelquefois leur couleur.

L'opercule montre deux sortes de taches; les unes sont analogues à celles du manteau, et paroissent seulement d'une manière plus vague; les autres, que nous n'avons encore remarquées que dans l' $A$. depilans, semblent devoir foumir un assez bon caractère spécifique; celles-ci sont noires ou du moins très-foncées, longues et étroites; mais ce qu'elles ont de plus particulier; c'est qu'elles rayonnent autour de l'ouverture centrale de l'opercule.

Le pied est d'un brun assez clair, offrant parfois des teintes bleues aux endroits où il se contracte.

La coquille est de forme oblongue, mais peu élargie dans son milieu; elle est assez concave, surtout à sa partie postérieure; sa texture est comme cornée, avec une légère couche calcaire à son sommet; celui-ci est épais, recourbé, et la 
partie de la coquille qui l'avoisine à gauche est déprimée; l'échancrure est courte et placée un peu en arrière; sa couleur est la même en dessus qu'en dessous, c'est un fauve ou jaune de succin avec une légère teinte roussâtre au milieu; le sommet est en partie blanc. Les stries longitudinales sont beaucoup plus apparentes que celles qui sont transversales.

L'A. depilans nous a donné lieu d'observer avec beaucoup de facilité les liqueurs dont nous avons parlé dans nos généralités: la liqueur blanche et celle qui suinte du manteau nous ont semblé très-abondantes; quant à celle qui provient de l'opercule, et qui, comme dans toutes les autres espèces, est d'une belle couleur de laque, nous l'avons trouvée un peu moins abondante que dans I'A. fasciata. C'est surtout dans les liqueurs de cette espèce que l'on trouve l'odeur forte que l'on attribue généralement aux Aplysics. Cette odcur, qui se manifeste promptement lorsqu'on irrite l'animal, et qui affecte tout ce qui l'entoure, tient un peu de célle du nusc, et n'est point désagréable quand elle n'est encore que foiblement répandue, mais bientòt elle acquiert une telle force qu'elle devient nauséabonde et presque insupportable. Ayant manié plusieurs de ces animaux, nous nous trouvâmes encore incommodé de cette odeur après plusieurs jours et malgré toutes les précautions que nous prîmes pour nous en débarrasser. D'après cela on doit conjecturer que c'est à cette propriété quel' $A$. depilans doit la mauvaise réputation qu'elle n'a cessé de conserver, et les exagérations auxquelles on s'est trop empressé d'ajouter foi.

Habilution. Cette espèce appartient aux rives de la Méditerranée, à nos côtes de l'Ouest, et à celles de la Manche et du Nord de l'Angleterre. Nous l'avons rencontrée en grande quantité avec l'A. fasciata, à marée basse, sous les rochers ou dans les anfractuosités de l'ancienne digue de Richelieu, à La Rochelle. Ces leux espèces vivent ensemble; mais nous ne les avons jamais trouvées accuuplées.

Cabinet d'Anatomie du Jardin des Plantes, AprySIE, $n^{\circ} 24$.

25. A. POLIAN A, delle Cinaje. Pl. XV bis., f. 2.

Delle Chraje, Giorr. med. uap. d'Inspruck; et Memorie sulla storia e nolom. delle Aplysie.

Corpore oblongo, levi, castaneo, anticè clepresso, posticè obtuso; foramine dorsi amplissimo; bran- chiis ultra caudam protentis; membranis parvis; siphone brevi, dentato, supernè erccto; pede dilatato.

Testâ? ?.....

Animal, oblong, élargi en avant, obtus en arrière; le manteau de couleur châtain ou carmélite; l'ouverture de l'opercule très-grande; les branchies dépassant l'extrémité caudale; les lobes petits; le siphon court, dentelé et dirigé en dessus; le pied grand. Longueur $0,172$.

Coquille?.....

Description. Voici la description que M. delle Chiaje nous donne de cette belle espèce, dont on lui doit la découverte : "Cet animal, dit-il, a les "lèvres blanches; les tentacules antérieurs ne sont " pas très-longs et nullement crêpés; l'ouverture " qui donne issue au membre génital est confor"mée comme à l'ordinaire, et le sillon qui réunit "les deux sexes est entièrement blanc. Les yeux " sont placés en avant des tentacules supérieurs; " le cou est débordé de chaque côté, et en avant, "par le pied, et les lobes, très-peu larges, sont - réunis à leur partie postérieure, où ils sont très"élevés au-dessus de la queue; ces lobes couvrent "à peine une partie de l'opercule, ce qui fait qu'ils " ne doivent que difficilement remplir les fonctions "qu'ils exercent dans les $A$. leporina (depilans), "fasciata, Camelus, etc.

"La membrane qui enveloppe la coquille pos" sède une très-large ouverture dont je n'ai point "vu d'exemple dans les autres espèces; le siphon, - un peu grand, dentelé et ayant l'anus à sa nais"sance, est redressé en dessus; les branchies ne "diffèrent en rien de celles des autres espèces, - seulement elles sunt longues et dépassent l'ex" trémité de la queue. L'A. poliana a-le pied un "peu plus large que les autres espèces, et sem"blable, sous quelques rapports; à celui de la "Bulla lignaria; en avant il est divisé en lobes "par des sinus, et dépasse beaucoup la bouche; " il est pareillement plus cléveloppé sur les côtés; " mais dans sa partie postérieure il se rétrécit in" sensiblement, cn se portant à environ un poizce "au-delà du siphon. La partie inférieure est très* lisse et de couleur violet-brun. Le manteau pré" sente, par l'effet de l'humeur qui s'échappe à tra"vers ses pores, une teinte semblable à celle que " produit la terre d'ombre. Ce mollusque mourut "quelques heures après avoir été tiré de la mor. M. delle Chiaje a joint à son texte une bonne 
figure qui suppléera à ce qui peut manquer dans sa description; cependant nous regrettons encore pour l'A. poliana, comme pour les autres espèces qu'il a décrites, de ne rien trouver de caractéristique au sujet de la coquille que contient l'opercule. Gette pièce, dit-il seulement, est osseuse.

Habitation. L'A. poliana habite les mers de Naples avec $\mathrm{l}^{\prime} A$. neapolitana. Il paroît qu'elle y est assez rare.

\section{A. FUSCA, Tilesios. Pl. XVIII, f. I. Voyage de Crusenstern.}

Corpore gibboso, oblongo, fusco, maculato; foramine dorsi radiato; pede oblongo, angusto; membranis mediocriter dilatatis.

Testâ fragili, flexibili....

Animal gibbeux, oblong, brun et tacheté; l'ouverture de l'opercule radiée; le pied oblong, étroit ; les lobes médiocrement larges. Longueur o,ogo.

Coquille fragile, flexible.....

Description. Nous empruntons cette espèce à la relation du voyage de l'Amiral Crusenstern, où elle est figurée et trop brièvement décrite. Voici à quoi se borne la description de M. Tilesius, Le dos est uni, gibbeux, tacheté, avec des lobes s'élevant de chaque côté. La partie supérieure est revêtue d'un opercule au milieu duquel est une ouverture dont les bords sont radiés; le pied est oblong, étroit, renflé et réfléchi à chaque extrémité; il montre une échancrure en avant, tout près de la bouche; les tentacules sont plissés sur leurs bords et en forme d'oreilles.

Habitation. Ce mollusque vient des mers de la Chine, où il habite près des rivages parmi les fucus et les éponges.

27. A. PUNCT AT A, Cuvier. PI. XVIII, f. 2-4. Ann. du Mus., 3, p. 295, pl. 1, f. 2-4; et Règne anim. t. II, p. 398. Deroissy, Moll., p. $x 72, n^{\circ} 4$.

Aplysia punctata, Férussac, Tab. syst., p. xxx; et Dict. class. d'hist. nat., tom. I, p. 476. Brativilue, Journ. de phys., janvier $x 823$, p. 287 ; et Dict. des sc. nat., au mot Lièvre marin. Paxraudeau, Moll. de Corse, p. 97 , $n^{\circ}$ I92.

Laplysia punctata, LaMaRcK, An. s. vert., t. VI, $2^{\mathrm{e}}$ parti, p. 40 .
Aplysia Cuvieri, nelue Chiaje, Mem. del regno di Napoli, e giorn. med. nap. dInspruck.

Corpore elevato, oblongo, posticè acuto, levigato, nigro-purpurescente punctulisque pallidis cooperto; tentaculis posterioribus elongatis; operculo oblongo, aperturâ in medio ovatâ.

Testâ oblongâ, concavâ, membranaceâ, succineâ; incisurâ quasi posteriore, brevi, leviter arcuatâ; rostro parvo.

Animal. Corps très-bombé, oblong, aigu en arrière, lisse et de couleur noir-pourprée, avec une grande quantité de petites taches pâles; tentacules postérieurs allongés; opercule oblong, avec une ouverture ovale au milieu. Longueur o, Ioo.

Coquille oblongue, concave, membraneuse, de couleur de succin; l'échancrure presque en arrière, courte, peu arquée, le rostre petit. Longueur 0,034 .

Description. L'Aplysie ponctuée a été établie par MI. Cuvier, pour une espèce que nous avons retrouvée dans la Méditerranée, où elle est trèscommune, surtout sur les côtes de Provence. Elle est de grandeur moyenne; son corps est très-renflé, aigu en arrière, un peu allongé en avant. Sa peau est lisse et varie parfois de couleur; dans quelques individus elle est brunåtre, mais le plus ordinairement on la trouve noire mélangée d'une teinte pourprée: du reste elle est dans tous les cas tachetée d'une grande quantité de petits points pâles. La surface de l'opercule ne montre que peu de ces taches, et quelquefois pas du tout. Le manteau est très-élevé en arrière; les lobes sont assez dilatés, mais moins cependant que dans plusieurs autres espèces; les tentacules postérieurs sont longs, et l'opercule, qui est de forme oblongue, porte une ouverture ovale à son milieu; le siphon est assez allongé; le pied est calleux, et ses bords paroissent souvent très-irréguliers et boursouflés.

La coquille est passablement concave; sa couleur est celle du succin, et sa texture est membraneuse; elle est oblongue; l'échancrure est très en arrière et peu profonde; le sommet est petit.

Habitation. La Méditerranée. Sur les côtes de Provence elle est très-commune, et se trouve avec l'A. fasciata. Les pêcheurs la distinguent fort bien.

Cabinet d'Anatomie du Jardin des Plantes, APLYSIE, $\mathbf{n}^{\circ}$ 27. . 
28. A. LONGICORNIS, Nobis. Pl. XIX, f. I-4.

Corpore oblongo, valdè elevato, posticè acuto, levigato, luteo-virescente, pallido; tentaculis posterioribus longissimis; aperturâ dorsi leviter dilatatá; pede angusto.

Testâ oblongâ, quasi rolundâ, valdè concavâ, cxili, membranaceâ, pellucidâ, succineâ; incisurâ quasi uullä; rostro minimo.

Animal. Le corps est oblong, très-élevé vers le dos, pointu en arrière; le manteau est lisse, de couleur jaune-verdâtre pâle; les tentacules postérieurs sont trèsilongs; louverture dorsale peu large; le pied étroit. Longueur o,ogo.

Coquille. Oblongue, presque ronde, très-concave, mince, semblable à une pellicule transparente, succinée; l'échancrure presque nulle; le rostre très-petit. Longueur o, o 16.

Description. Cette espèce est d'assez petite taille; elle a le dos extrêmement bombé; le manteau se relève beaucoup de toute part, mais surtout en arrière, en sorte que l'ouverture dorsale est peu dilatée et située tout-à-fait èn-dessus. Les lobes sont assez étroits, et, par leur réunion en arrière, ils brident fortement l'opercule. Celui - ci est grand, et l'ouverture de sa membrane supérieure est large et oblongue; la partie antérieure du mollusque est assez allongée; les tentacules antérieurs sont grands, et n'offrent zien de particulier, mais ceux qui les suivent ont cela de remarquable qu'ils sont très-allongés et pointus. La couleur génẻrale de $l^{\prime} A$. longicornis est un jaune-verdâtre pâle; le manteau est lisse et mollasse; le pied est un peu étroit et très-calleux.

La coquille est de couleur de succin, membraneuse et transparente, ayant l'apparence d'une pellicule; elle est presque toute ronde et très-concave; son échancrure est à peine sensible, et son sommet très-peu formé.

Habitation. Elle se trouve dans la Méditerranée, particulièrement sur les côtes de France. Une partie des individus qui sont au Jardin des Plantes y ont été envoyés par M. Polidor Roux.

Cabinet d'Anatomie du Jardin des Plantes, A PLY$\operatorname{sir}, \mathrm{n}^{\circ} 28$.
29. A. FERUSSAC11, Nobis, Pl. XIX, f. 6.9.

Corpore oblongo, valdè convexo, posticè brevi, fusco-livido, maculis obscuris natato; aperturâ operculi valdè dilatatâ; pede angusto.

Testâ quasi rotundâ, succineâ, valde exili, pel. lucidả; incisurâ quasi nullâ; rostro minimo.

Animal oblong, très-bombé et court en arrière; son manteau est de couleur brun-livide, varié par des taches foncées, grandes et très-irrégulières; l'ouverture de l'opercule est très-grande; le pied est étroit. Longueur 0,035 .

Coquille presque ronde, de couleur de succin, semblable à une pellicule mince et transparente; l'échancrure est presque nulle; le sommet est trèspetit. Longueur 0,008 .

Description. Cette espèce, qui étoit dans la collection de M. de Férussac, et que ce savant a bien voulu mettre à notre disposition, se distingue beaucoup des autres par sa petitesse; elle est oblongue, très-bombée, un peu courte en arrière et assez allongée en avant; son manteau est très-haut, surtout à la partie postérieure, et ses lobes sont un peu étroits; l'opercule est oblong, et sa membrane largement ouverte; le pied est étroit. La couleur générale est un brun-livide, varié par de grandes taches noires de forme très-irrégulière.

La coquille, semblable à une pellicule mince, pâle et diaphane, est de forme presque ronde; son échancrure est à peine visible, de mêrne que son sommèt. Habitation?

Cabinet de M. le baron d'Audebard de Férussac.

3o. A. VIRESCENS, Risso. Pl. XIX, f. 5.

Aplysia virescente, Rısso, Hist. nat. de l'Europe mérid., IV, $\mathrm{n}^{\circ} 96$; et Aplysia virescens, même volume, pl. $\mathbf{I}$, f. ro.

Aplysia unicolor? Risso, Journ. de phys., 87, p. 374 .

Corpore ovato, virescente, obscure marmorato; capite proeminente.

Testâ ovatâ, vitreâ, pellucidâ, sulcis tenuibus concentricis sculptâ, ad dextram sinuatä. Risso.

Animal. Le corps est de forme ovale, de couleur verdâtre marbré de taches foncées; la tête est proéminente. Longueur o,o3o.

Coquille ovale, vitrée, transparente, portant une échancrure à gauche, et ornée de stries fines et concentriques. Longueur? 
Description. N'ayant pas une connoissance suffisante de cette espèce, dont MI. Risso a fait mention dans son livre sur les mollusques de l'Europe méridionale, nous ne pouvons la caractériser d'une manière convenable et qui cadre avec les descriptions des autres espèces. Nous nous contenterons de transcrire la phrase de M. Risso, de même que nous avons copié la figure. Nous ferons remarquer en même temps que ce naturaliste l'a décrite dans son texte sous le nom de $A$. virescente, tandis qu'il la représente, f. 1 o, pl. I, sous le nom de $A$. virescens. "Le corps de cette espèce, dit-il, est d'un " beau vert marbré de brun; la tête proéminente, " affectant une forme quadrangulaire; les tentacules " verdàtres, les yeux noirs, la partie postérieure " oblongue, le pied d'un vert-clair.

"Coquille ovale, vitrée, translucide, sculptée "par de très-fins sillons concentriques, sinuée à "droite. v

Habitation. Elle habite la Méditerranée, parmi les fucus. On la trouve au mois d'avril.

3o bis. A. ROSE $A$, Rathiк. Pl. XXIII, f. 6,7 . Mémoires de la société d' hist.nat. de Copenhague, t. V, I ${ }^{\text {re }}$ part., pag. 85 , pl. in, f. $\mathrm{n}, \mathrm{a}, \mathrm{b}$.

Corpore oblonga, posticè acuto, levigato, roseo, punctulis albidis badiisque ornato; tentaculis anterioribus acutis, posterioribusque obtusis; membranis angustis; foramine operculi dilatato.
Testâ corneâ, oblongâa, leviter concavâ, rubrobruneâ.

Animal. Oblong, peu allongé antérieurement, pointu en arrière; manteau lisse de couleur rosée, avec de petites taches blanches et brunes; les tentacules antérieurs pointus, les postérieurs obtus; lobes peu larges; membrane de l'opercule largement ouverte au milieu. Longueur 0,032 .

Coquille cornée, oblongue, peu concave, de conleur rouge-brun. Longueur o,oro.

Description. Cette espèce, décrite par M. Rathke, paroît fort distincte de toutes les autres. Elle est beaucoup moins bombée que les précédentes; elle est oblongue, peu allongée en avant et pointue en arrière. Les tentacules antérieurs sont aigus et les postérieurs comme tronqués, Les lobes sont assez étroits, et l'op̉ercule est oblong et muni dans son centre d'une large ouverture qui laisse apercevoir la coquille. Le manteau est lisse et de couleur rosée, avec de petites taches blanches et brunes très-nombreuses. Le pied est large et blanchâtre.

La coquille paroît oblongue, sans échancrure bien prononcée; sa nature est cornée, transparente, et sa couleur rousse. Elle paroît peu concave.

Cette espèce est représentée avec son pennis hors de sa cavité.

Habitation. Aux environs de Christiansund. 


\section{deuxième sous-genre. NOTA R CHES; Gürer.}

\section{Notarchus, Cuvier; Laplisia, Bosc; Aplysia, de Blainville, Férussac; Actoon,}

Férussac; Bursatella, Audouir; Aclesia, sur nos planches xx, xxi et xxII (I).

Animal muni d'une fente dorsale très-petite et quelquefois oblique; le pied allongé et assez étroit; les branchies souvent fort longues, et pouvant se porter au dehors de la cavité; l'opercule rudimentaire ou nul.

\section{Test nul.}

Le caractère le plus important du sous - genre Notarche consiste dans l'absence du test rudimentaire dans l'opercule. Il résulte de cette disposition que l'opercule est fort petit, et souvent à peine apparent, ou plutôt on peut dire qu'il n'en existe pas, car on ne peut plus donner ce nom à un léger repli de la membrane que nous avons remarqué dans quelques individus, et qui ne couvroit point la cavité branchiale. Dans les Notarches, l'ouverture dorsale est généralement petite, semblable à une fente, et quelquefois placée un peu à droite de la ligne médiane; ou bien sẹlement oblique, allant d'arrière en avant et de gauche à droite. On conçoit facilement que le canal qui réunit la vulve à la verge entraine cette dernière disposition. Le panache des branchies se montre généralement plus allongé, et se porte souvent au dehors de la cavité qui le renferme. Les lobes sont extrêmement petits, presque nuls, et par conséquent tout-à-fait impropres à la natation. Le pied se distingue de celui des Aplysies proprement dites, en ce qu'il est plus étroit et en même temps plus allongé; il arrive même quelquefois qu’il se prolonge en arrière en une sorte de queue assez longue. Les tentacules antérieurs sont moins dilatés, sans doute parce que ces animaux ne s'en servent point pour la natation; les postérieurs se montrent assez effilés dans certaines espèces, tandis que dans d'autres, au contraire, ils sont courts et obtus.

Ces animaux, comme nous venons de le dire, ne sauroient nager, et, si l'on examine leur pied, on voit qu'ils ne doivent point, non plus, ramper avec facilité; cet organe ne paroît leur servir, en quelque sorte, que pour saisir les tiges et les feuilles des plantes marines, à la manière des Scyllées et des Téthys, et c'est en effet sur ces plantes qu'ils vivent. Nous en avons plusieurs fois rencontré, à quelques centaines de lieues en mer, errant à la merci des courants sur différentes sortes de fucus.

Nous rapportons à ce sous-genre l'Aplysie verte de M. Bosc, parce que ce naturaliste croit qu'elle n'a point de test; cependant nous avouons qu'elle ne possède pas tous les caractères que nous venons de signaler comme concourant, avec l'absence de la coquille, à la formation de ce sous-genre. Ce n'est donc qu'avec incertitude que nous la plaçons à la suite des Notarches.

(1) Nous avons déja dit que nous avions commis une faute en donnant à ce second sous-genre le nom d'Aclésie; nous ignorions alors que les mollusques qui le composent ne sont autres que des Notarches de M. Cuvier; henreusement nous étions encore à temps de rectifier cette erreur dans notre texte. 


\section{3r. A. SAVIGNANA, Férussac. Pl. XX.}

Bursatella savignana, Addodin, Explic. somm. des planches de moll. de l'Egypte, p. I6.

Corpore ventricoso, ovato; anticè obtuso, posticè acuto; appendicibus numerosis, digitatis, regulatimque dispositis; caudâ strangulatâ, triangulari, subulatâ, maculatâ; aperturâ dorsi minimá; tentaculis anterioribus minimis; tentaculis posterioribus brevibus, digitatis; griseo-virescente.

Animal. Le corps est ovale, bombé et ventru; le cou allongé et obtus; la partie postérieure aiguë et séparée par une sorte d'étranglement; le manteau de couleur gris-verdâtre, couvert d'appendices nombreux digités et symétriquement disposés; l'ouverture dorsale très-petite; les tentacules antérieurs très-petits; les postérieurs courts et digités; le pied oblong, allongé, pointu et prolongé en arrière. Longueur o, 115.

Description. Cette curieuse espèce, que nous ne connoissons que par la belle planche de la Description de l'Égypte, où M. Savigny l'a fait représenter, et par la description que M. Audouin vient d'en donner dans l'explication sommaire des planches de ce magnifique ouvrage, se distingue très-facilement, même dès le premier aspect, de toutes les autres, mais notamment de celles de notre premier sous-genre.

Sa forme générale est ovale, bombée un peu en arrière du milieu du dos, et très-ventrue sur les côtés. Son cou est lárge et médiocrement allongé; la tête, qui est également large et se termine presque carrément en avant, en est séparée par un étranglement bien apparent; en arrière la disposition de l'animal offre des caractères non moins remarquables; cette partie est triangulaire, pointue et prolongée en forme de queue; mais ce qui la distingue surtout, c'est qu'elle est aussi séparée du corps par une sorte d'étranglement trèssensible en dessus et sur les côtés; sa surface est parsemée de petites oscules noirâtres, et hérissée d'appendices disposés de la manière suivante: quatre de ces appendices occupent la ligne médiane, et se montrent digités; trois autres sont placés sur les lignes latérales, mais parmi ceux-ci le plus antérieur de chaque côté est seul digité, tandis que les autres sont simples, coniques, courbes et dirigés en arrière; le manteau offre luimême sur toute sa. surface un grand nombre de ces appendices digités, diversement conformés et de différentes tailles, mais qui paroissent cependant affecter dans la place qu'occupent les principaux une disposition symétrique. Ces appendices, qui ont une couleur verdàtre un peu brune, montrent à leur base quelques petites taches noirâtres. La couleur générale du manteau est un gris-verdâtre. L'ouverture dorsale est centrale; elle est ovale et très-petite; elle laisse apercevoir le peigne branchial, qui, comme nous l'avons observé sur une espèce analogue, se porte sans doute au dehors pendant les mouvements de l'animal. On distingue encore dans la figure que nous empruntons à M. Savigny, un rudiment d'opercule qui ne paroît formé que par un repli de la membrane. Les tentacules antérieurs sont très-petits et semblables à des ailerons; les postérieurs sont courts, obtus, ouverts à leur sommet et hérissés d'appendices. Ces deux derniers se confondent facilement avec trois groupes d'appendices digités, placés I'un sur le milieu de la tête, et les deux autres plus en avant et tout près des tentacules antérieurs. Le pied offre aussi des caractères importants; il est oblong, assez étroit, distinct du manteau par un rebord bien tranché et par une série nombreuse de petits appendices simples et dirigés en arrière; sa couleur est moins foncée que celle du manteau; en avant il se distingue par un repli qui est assez analogue à un caractère bien remarquable que nous avons observé sur le Notarchus gelatinosis, et dont nous parlerons en décrivant cette espèce, et en arrière il est plissé trans. versalement et prolongé en pointe presque jusqu'au bout de la queue.

Cette espèce, suffisamment caractérisée, est de toutes celles dont nous avons une connoissance suffisante, celle qui se lie le mieux aux espèces du premier sous-genre, tant par son ouverture dorsale, son petit opercule, que par son pied encore assez large, et susceptible de ramper avec facilité. Les détails anatomiques que M. Savigny a joints à sa planche, et que nous avons copiés, ajoutent encore beaucoup à la connoissance de ce mollusque.

La symétrie des appendices paroît, d'après la figure de l'A. savignana, être un caractère constant; c'est pourquoi nous l'avons présentée comme tel : quant à l'appendice du milieu de la tête, nous ne le considérons point comme un tentacule, non plus que ceux qui sont plus en avant; ce ne sont sans doute que de petits organes semblables à ceux qui couvrent le reste du corps de cette espèce et 
de la suivante, et qui nous rappellent encore les appendices de la première section des Aplysies proprement dites (Voir l'explication de la planche xx).

M. Audouin, dans l'ouvrage que nous avons cité, fait de l'A. savignana une Bursatelle, et des Bursatelles de MI. de Blainville un sous-genre du genre Aplysie. 11 en résulte qu'il considère cette espèce absolument comme nous, c'est-à-dire qu'il croit qu'elle doit appartenir au genre Aplysie; la seule différence, c'est qu'il forme son sous-genre avec une autre dénomination que celle que nous avons adoptée. On peut soupconner de là que le genre Bursatelle de M. de Blainville n'est autre chose que le genre Notarche de M. Cuvier; rapprochement que nous serions assez porté à adopter, mais que la connoissance trop incomplète du genre Bursatelle ne nous permet pas encore d'opérer.

Habitation. Elle a été recueillie sur les côtes d'Égypte, par M. Savigny, à l'époque où les sciences exploitoient ce beau pays, à la suite de nos armées victorieuses.

\section{A. PLEII, Nobis. Pl. XXI.}

Corpore ventricoso, ovato, anticè obtuso, posticè acuto, obscuro-virescente, appendicibus numerosissimis digitatisque hirsuto; aperturâ dorsi minimâ, angustâ; pectine branchiarum valdè elongato; pede angusto.

Animal. Corps ovale, bombé et ventru; cou assez court et élargi ; tête obtuse; partie postérieure un peu courte et aiguë; manteau de couleur verdâtreobscur, hérissé d'un grand nombre d'appendices digités; ouverture de la cavité dorsale petite; tentacules antérieurs aplatis; tentacules postérieurs coniques; pied très-étroit. Longueur o, 125.

Description. L'A. Pleii a les plus grands rapports avec la précédente; cependant elle s'en distingue par des caractères bien importants : comme elle, sa tête est séparée du cou par un léger étranglement, et son manteau est de couleur verdâtre et hérissé de nombreux bouquets d'appendices ramifiés ou digités ; comme elle son pied est bien distinct du manteau, et bordé par un repli et un nombre considérable d'appendices simples; mais cette tête porte deux tentacules larges et aplatis; ces appendices n'affectent pas autant une disposition symétrique, et le pied, plus étroit dans toute sa longueur, ne montre point en avant le repli dont nous avons parlé en décrivant l' $A$. savignana, et ne se prolonge pas en queue en arrière. Dans l'A. Pleii les tentacules postérieurs se montrent coniques et dépouillés d'appendices; enfin la partie qui termine le mollusque en arrière paroît différente; elle est simple comme dans toutes les autres espèces, beaucoup plus courte, et n'offre point cette espèce d'étranglement qui caractérise si bien la première, ni ces trois séries d'appendices si régulièrement disposés. Nous ajouterons encore que l'ouverture dorsale est moins large en avant; que ses bords forment deux petites lèvres ou rudiments de lobe natatoires, et que les branchies, très-allongées au côté droit, se portent à l'extérieur de la cavité, à la volonté de l'animal.

Habitation. Elle appartient aux mers des Antilles, où nous l'avons rencontrée sur les tiges des fucus, qu'elle paroît ne jamais quitter. Elle a été envoyée au Jardin des Plantes, par M. Plée, qui l'a également recueillie. dans ces mers.

Cabinet d'Anatomie du Jardin des Plantes, AprySIE, $\mathbf{n}^{\circ} 32$.

32 bis. A. GELATINOSA, Nobis. Pl. XXIII, f, I-5.

Notarchus, Cuvier, Riègne anim., tom. II, p. 398 .

Notarchus Indicus, Schweig. Fénussac, Tabl. syst., p. xxx.

Notarchus Cuvieri, oe Buanville, Dict. des sc. nat., t. XXXV, p. 16I; et Manuel de malacol., p. 473 , pl. xuri, f. 7 . Desmayes, Dict. class. d'hist. nat., t. II, p. 600 .

Corpore oblongo, convexo, anticè brevi, posticè obtuso, levigato, obscuro-virescente ; aperturâ dorsi minimâ, obliquâ; pede valdè angusto, striato, posticè acuminato, margine distinctâ, longitudinaliter sulcato, ad marginem anteriorem transversim biplicato.

Animal. Corps oblong, très-bombé et ventru, peu allongé et obtus à chaque extrémité; manteau lisse, verdâtre-obscur, et armé sur le dos et en arrière de quelques petites aspérités; ouverture dursale très-petite et oblique; tentacules comme dans l'espèce précédente; pied très-étroit, acuminé en arrière, à bords séparés du manteau, strié en travers, muni d'un sillon longitudinal, et en avant d'un double repli transverse. Longueur o, 038 .

Description. L'A. gelatinosa; décrite pour la première fois par M. Cuvier, avoit été constituée en 
genre, sous le nom de Notarche, par ce savant; nous avons cru devoir, comme nous l'avons déja dit, la réunir aux Aplysies, en l'établissant en sousgenre sous le nom qui lui avoit d'abord été affecté. Ayant pu à loisir étudier les deux individus que possède le Cabinet d'Anatomie du Jardin des Plantes, et qui sont les mêmes qui ont servi aux observations de M. Cuvier, nous pouvons en donner une description détaillée. Le nom spécifique que nous lui avons imposé étoit sur le bocal qui les contenoit, et sans indication d'auteur.

L'A. gelatinosa est de petite taille; elle est de forme oblongue, très-gonflée et chargée de boursouflures; son cou est très-court, un léger étranglement le sépare de la tête, et celle-ci, de forme globuleuse, est ohtuse en avant; la partie postérieure est également courte et arrondie; le manteau est lisse, cependant on distingue quelques petites aspérités assez symétriquement disposées en arrière de la fente dorsale; sa couleur est, autant que nous avons pu en juger sur l'animal très-bien conservé dans l'esprit-de-vin, d'un verdâtre-obscur, comme dans un grand nombre de ces animaux; il ne nous a pas été possible de reconnoître des traces de taches; l'ouverture dorsale, semblable à une fente, est très-petite, et située plutôt en avant qu'en arrière de la sommité du dos; mais ce qui est bien remarquable, c'est que cette ouverture, aulieu de suivre la ligne médiane, s'en écarte pour suivre une direction oblique qui est la même que celle du canal qui réunit les organes de la génération, et qui se montre immédiatement en avant; ce caractère, que nous n'avons point eu lieu de signaler dans les espèces du sous-genre Notarche que nous avons déja décrites, se reproduira encore. Le panache des branchies est assez allongé, et se porte facilement et fréquemment au dehors de la cavité; la membrane operculaire est réduite à l'état le plus rudimentaire; les tentacules n'offrent rien de remarquable, et les yeux, très-visibles à la vue simple, sont placés comme dans les autres espèces. Le pied de ce mollusque offre à lui seul des caractères qui suffiront peut-être pour en rendre la détermination facile; il est extrêmement étroit, mais surtout en arrière, où il se termine en pointe; ses bords sont entièrement indépendants du manteau, et un sillon longitudinal en partage la surface en deux parties égales : il résulte de cette disposition que le pied peut se fermer complètement en rapprochant et appliquant bien ces deux moitiés l'une contre l'autre; c'est sans doute par ce moyen que cette espèce saisit les feuilles sur lesquelles elle se fixe, et cette disposition annonce bien que l'animal ne peut ramper qu'avec beaucoup cle difficulté; la surface de ce pied est striée transversalement, et ses bords sont finement plissés; en avant nous avons remarqué un caractère qui nous a offert quelque analogie avec ce que l'on voit au même en'droit du pied dans la figure de l'A. savignana, c'est un double repli de la membrane placé dans une direction transverse, et qui, par sa forme, nous rappelle la petite ventouse que l'on observe à la nageoire ventrale des Carinaires et des Firoles : peutêtre cet organe a-t-il dans ce mollusque la même destination que celui auquel nous le comparons dans ceux que nous venons de citer, c'est-à-dire qu'il serviroit à fixer l'animal à la surface des corps; c'est un fait qu'il seroit intéressant d'approfondir.

Habitation. L'A. gelatinosa habite l'île de France, d'où elle a été rapportée par M. Mathieu.

Cabinet d'anatomie du Jardin des Plantes, ApLySIE, $n^{\circ} 32$ bis.

\section{A. CITRINA, Nobis. P1. XXII, I, 2.}

Corpore in medio valdè gibboso, elongato, ad extremitates acuto, substranslucido, citrino cum maculis albidis asperitatibusque minimis rarè sparsis; aperturâ dorsi minimâ, angustâ, obliquâ; pede angusto.

Animal. Très-bombé sur le milieu du dos; effilé et aigu aux deux extrémités ; manteau un peu translucide, jaune, avec de petites taches blanches et de très-petites aspérités; ouverture dorsale trèspetite, étroite et un peu oblique; pied très-étroit. Longueur $0,025$.

Description. Cette jolie espèce, aussi élégante par sa forme que par le bel éclat de son manteau, est la plus petite du genre. Elle est très-bombée dans son milieu; son cou est allongé, et sa tête porte des tentacules qui n'ont rien de remarquable; la partie postérieure du corps est effilée et aiguë; le manteau est jaune couleur de gomme gutte, et montre beaucoup de taches blanches et de petites aspérités; sa transparence est telle que l'on distingue quelques-uns des viscères, et particulièrement le cour, dont on peut compter les battements à travers són épaisseur. L'ouverture dorsale est petite, étroite, et oblique un peu, comme dans l'espèce précédente, dans le sens du canal de la verge; nous n'avons point vu sortir les branchies, 
quoique nous ayons, à loisir, observé l'animal à l'état de vie. La membrane de l'opercule nous a semblé nulle; le pied est long et très-étroit. L'A. citrina répand une liqueur de la même couleur qu'elle.

Habitation. Nous avons trouvé cette jolie espèce au milieu de l'Océan équatorial, sur des masses flottantes de fucus natans, où, par sa couleur, elle sembloit confondue avec les feuilles de ces plantes; de nombreuses Scyllées et plusieurs Éolides vivaien t avec elles. L'individu que nous venons de décrire est le plus gros de ceux que nous avons rencontrés.

34. A. LIMACINA, Buainville. PI. XXII, f. $6,7$. Journ. de phys., 96, janv. 1823, p. 287 , pl.? f. 10 ; et Dict. des sc. nat., au mot Lièvre MARIN.

Corpore limaciformi, oblongo, levigato, complanato, anticè oltuso, posticè acuto, ad basim depresso, obscuro-virescente; aperturâ dorsi anticè angustâ; pede valdè dilatato.

Animal limaciforme, de forme oblongue, obtus en avant, aigu en arrière, aplati et déprimé tout autour de la base; le manteau est lisse et de couleur verdàtre-obscur; l'ouverture dorsale est étroite en avant, bâillante en arrière; le pied trèslarge. Longueur 0,035.

Description. Cette espèce a été décrite pour la première fois et nommée par M. de Blainville, sur des individus appartenants à la collection de M. Brongniart; nous l'avons depuis observée vivante sur les côtes de la Méditerranée, et nous n'avons pas eu de peine à la reconnoître, ses caractères étant parfaitement établis. Elle est, comme le dit M. de Blainville, limaciforme; son corps est oblong, pas beaucoup plus large en arrière du dos qu'en avant; sa partie antérieure est obtuse et la postérieure très-peu allongée et aiguë; son dos est à peine bombé, et on peut lui assigner ce caractère unique jusqu'ici, qu'elle est généralement aplatie. Son pied étant plus large qu'elle, il s'ensuit que la base de son corps présente tout autour une dépression assez visible, analogue à ce que nous avons déja signalé dans plusieurs espèces, mais qui paroît constituer un caractère particulier pour toutes les espèces de la seconde section du premier sous-genre. Les tentacules n'offrent rien de particulier; le manteau est très-lisse et de couleur verdâtre-obscur; l'ouverture dorsale est assez longue, très-étroite en avant et bâillante en arrière; les branchies, qui ne nous ont pas paru devoir se porter à l'extérieur, sont à découvert dans la cavité, sans aucune apparence de membrane operculaire.

Habitation. Les rivages de la Provence.

35. $A$. NUDATA, Nobis. Pl. XXII, f. $3,5$.

Corpore convexo, ventricoso, oblongo, antice elongato, posticè acuminato, obscuro-virescente cum punctulis albidis rarè sparsis; aperturâ dorsi posticè dilatatâ; pede angusto.

Animal très-bombé et ventru, allongé et effilé aux deux extrémités; le manteau lisse, de couleur verdâtre-obscur et parsemé de quelques points pâles; l'ouverture dorsale assez longue, étroite et bâillante en árrière; le pied étroit. Longúeur 0,036 .

Description. L'A. nudata se distingue surtout par sa forme très-loombée, ventrue et l'allongement à peu près égal de ses extrémités antérieures et postérieures; la tête est petite, et les tentacules n'offrent rien de particulier; le manteau est parfaitement lisse, transparent, de couleur verdâtre un peu foncé, et parsemé autour de l'ouverture dorsale de quelques points blancs. Cette ouverture est assez longue, mais ses bords sont très-serrés et rapprochés en avant, tandis qu'au contraire, ils sont, comme dans l'espèce précédente, très-écartés en arrière. On conçoit que cette disposition est essentielle pour des animaux qui, n'ayant point de membrane operculaire, ne peuvent avoir de siphon pour servir à l'introduction de l'eau nécessaire aux branchies; celles - ci sont entièrement privées d'opercule, et ne paroissent point avoir assez de longueur pour pouvoir se porter hors de la cavité; le pied est très-étroit, et par conséquent ne déborde pas, comme dans l'espèce précédente.

Les œufs de cette espèce, qui forment une petite pelote semblable à celle que nous avons décrite en parlant de l' $A$. fasciata, s'en distinguent cependant par la finesse du tube qui les renferme, et par leur couleur blanche et transparente.

Habitation. Elle a été rencontrée dans la mer Pacifique, à peu de distance des îles Sandwich, sur des fucus entraînés par les courants à de grandes distances du rivage. C'est à MM. Quoy et Gaimard que nous la devons.

Cabinet d'Anatomie du Jardin des Plantes, APLYSIE, $\mathbf{n}^{\circ} 35$. 
36. A. $L O N G I C A U D A$, Quoy et Gamard. Pl. XXII, f. 8- Io.

Voyage de la corvette l'Uranie, 'To. II, p. 42 I, atlas, pl. Lxvi, f. 8 .

Corpore viride, punctis suprà cceruleo-rubris; cautuá longissimâ, acutâ.

Animal bombé, ventru et ovale; la partie antérieure allongée et la tête petite; la postérieure extrêmement allongée et aiguë; le manteau vert, avec des taches variées de rouge et dẻ bleu; l'ouverture dorsale petite, un peu en arrière et oblique; le pied très-étroit. Longueur 0,063 .

Description. Nous empruntons à MM. Quoy et Gaimard la description quiils donnent de cette jolie espèce : "Son cou, disent-ils, est assez allongé; ses "tentacules sont pointus, son corps ovalaire, l'ou"verture de ses branchies peu élargie, sa queue " excessivement longue, et sa couleur générale d'un "joli vert, parsemé de points rouges entourés d'un " cercle bleu de ciel, et cà et là de quelques autres " taches blanchâtres et bleues.

"Quoique cet animal ait été dessiné sur le vi"vant, ajoutent ces estimables naturalistes, il se " trouve cependant que certains détails ne sont pas "assez bien marqués, comme d'avoir le pied plus "relevé sur le dos; cela tient à ce que dans l'instant "où il a été dessiné, il embrassoit un fucus, et ne "pouvoit pas présenter ce caractère aussi saillant "que lorsqu’il rampe sur une surface plane.

"Cette élégante aplysie, ajoutent-ils encore, a " été prise sur des fucus, sous l'Équateur, dans les " parages de la Nouvelle-Guinée. Elle aura suivi " la plante sur laquelle elle étoit lorsque les flots "la détachèrent; car il n'est pas dans la nature de "ce gastéropode de quitter le rivage pour la haute "mer."

Nous pensons que ces savants sont dans l'erreur, et que cette espèce, soit qu'elle se trouve au large, soit qu'elle habite les rivages, ne vit pas autrement que sur les fucus; par conséquent, qu'il n'y a point dans leur dessin le défaut d'exactitude qu'ils croient devoir signaler, parce que le pied, comme nous avons eu lieu de nous en convaincre sur les individus conservés qu'ils ont déposés au Jardin des Plantes, est extrêmement étroit. Cette espèce n'habitant que sur les plantes marines, où, comme nous l'avons déja dit, elle ne se traîne que lentement, ne peut avoir le pied conformé précisément comme celles qui rampent sur les rochers, et il n'est pas extraordinaire qu'on la rencontre au large des terres.
Nous ne ferons plus qu'une seule observation au sujet de cet intéressant mollusque, c'est que ces naturalistes ont représenté dans leur dessin six tentacules, tandis que nous n'en avons rencontré que quatre sur les individus conservés que nous avons eus entre les mains : pour nous éclairer à ce sujet, nous avons interrogé sur ce fait, qui nous sem. bloit une erreur, M. Gaudichaud, l'un des savants voyageurs de l'expédition de l'Uranie; il nous a assuré que ce petit animal avoit trois paires de tentacules, et pour nous en convaincre, il nous a montré deux figures simplement esquissées quil avoit faites lui-même sur l'animal, et qui présentent d'une manière concluante ce singulier caractère. Ayant d'ailleurs remarqué quelques différences entre ces esquisses et la figure de l'atlas cité, nous avons jugé à propos de les copier, afin de ne négliger aucuns des renseignements qui peuvent concourir à faire reconnoître cette espèce exotique.

Habitation. Les eaux de la Nouvelle-Guinée, sur des fucus.

Cabinet d'Anatomie du Jardin des Plantes, ApLxSIE, $\mathrm{n}^{\circ} 36$.

37. A. VIRIDIS, Bosc. Pl. XXII, f. 2.

Laplysia viridis, Bosc, Hist. nat. des vers, t. I, f. $74, \mathrm{pl} .2$, f. 4; et Nouveau Dict. d'hist. nat. Aplysia viridis, de Rorssy, Buffor, t. V, f. I 73 ; Cuvier, Ann. du Mus., 3, p. $29^{5}$, et Mém. Anat.; Buarnvilue, Dict. des sc.nat., au mot Liève Marin, et Manuel de Malacol., p. 472. Actæon aplysiformis, Fénussac, Tab. syst., p. xxx, et Dict. class. d'hist. nat, t. I, p. 105.

Corpore oblongo, elongato, viride, punctulis rubris cooperto; cavitate dorsi grandi; membranis lateralibus elongatissimis, dilatatis, marginibusque pallidis; tentaculis anterioribus valdè dilatatis.

Animal. Corps oblong, allongé, de couleur verte, finement ponctué de rouge; le cou peu allongé; les tentacules antérieurs très-dilatés; les lobes très-longs, assez larges et bordés d'une teinte pâle; la cavité dorsale large. Longueur $0,020$.

Description. M. Bosc, à qui l'on doit la connoissance de cette espèce, la décrit ainsi : "Il n'a (ce "mollusque) que deux tentacules, n'est point vé"néneux, et probablement n'a pas d'os ou de test " intérieur. La tête de cet animal est antérieure" ment garnie de deux membranes transverses, "échancrées en leur milieu, et cachant la bouche “dans leur intervalle; elle a postérieurement deux 
" tentacules en forme d'oreille, placés en dessus "et devant les yeux. La membrane du corps est "verte, finement ponctuée de rouge; les bords plus "pâles et toujours repliés en dessus. Il s'élève au "plus à deux centimètres et est très-commun dans "les lieux vaseux."

Nous ajouterons quelques observations à la description de M. Bosc. Ce savant dit que l' $A$. viridis n'a que deux tentacules; cependant la figure qu'il donne en montre quatre conformés absolument comme dans le plus grand nombre des autres espèces; c'est-à-dire que les postéricurs sont en forme d'oreille et allongés, tandis que ceux de de- vant sont dilatés et en forme de cornet. Nous pensons donc qu'il ne faut point avoir égard au premier caractère indiqué par ce naturaliste. Quant à celui qui consiste dans la position des yeux, nous le repousserions également si nous ne savions pas quelle confiance on peut avoir dans les observations de M. Bosc. Nous dirons aussi combien il est à regretter qu'il règne de l'incertitude sur l'existence de la pièce testacée, car ce doute nous empêche peut-être de placer convenablement cette espèce.

Habitation. M. Bosc a trouvé l'A. viridis dans la baie de Charlstown (Amérique septentrionale), où elle habite les lieux vaseux.

\section{ESPECES INCERTAINES OU INSUFFISAMMENT CONNUES.}

\section{A. OCELLATA, Férussac. Pl. XXIV, f. 2-4.}

Le mollusque qui constitue cette espèce ne nous est connu que par un très-joli dessin de M. VanHasselt, communiqué à M. de Férussac par M. Tem minck. Il n'existe aucune description de ce petit animal, mais sa forme et la disposition du plus grand nombre de ses organes extérieurs nous ont donne l'idée qu'il devoit sans doute appartenir au genre Aplysie et peut-être au sous-genre Notarche. Voici la description que nous essaierons d'en donner. Le corps est très-bombé, arrondi et ventru; il se rétrécit et s'affaisse brusquement en arrière pour former une queue pointue et assez plate; en avant, le cou se montre un peu étroit et peu allongé; la tête est arrondie et présente deux paires de tentacules analogues à ceux des espèces de la seconde section du premier sous-genre. Ces tentatacules assez allongés sont aussi larges à leur extrémité qu’à leur base; ceux de devant ne sont point dilatés comme on le voit dans le plus grand nombre des espèces. Les yeux paroissent situés en avant des tentacules postérieurs comme dans toutes les Aplysies; le pied paroît étroit et allongé; le manteau est lisse, d'un beau jaune uniforme, et orné sur la partie dorsale de sept taches ocellées, bleues au centre et orangées à l'entour; ces taches sont disposées dans un ordre symétrique; le dessus n'offre aucune apparence de cavité dorsale ou de lobes.

La disposition du corps, de la tête et du pied, la forme, la position et le nombre des tentacules, la place qu'occupent les yeux; enfin, l’aspect général de ce joli mollusque, ne nous permettent pas de le repousser du genre Aplysie, quoique nous n'ayons aucune donnée sur la cavité branchiale, surtout si l'on considère que cette cavité a pu échapper aux investigations de M. Van-Hasselt, à cause de l'extrême petitesse de l'individu. Il nous souvient d'avoir eu beaucoup de peine à reconnoître ce caractère important sur notre $A$. citrina qui est de la même couleur, mais bien plus ğrosse; à plus forte raison a-t-il pu ne pas se manifester sur l' $A$. ocellata; d'un autre côté, la forme allongée et brusquement tranchée de la queue, l'apparence étroite du pied, les taches du manteau, semblables à celles de l'A. longicauda, et leur répartition symétrique sur le dos, jointes à la probabilité que la fente dorsale étoit infiniment petite, puisqu'elle n'a pas été aperçue, nous portent fortement à croire que cette espèce appartient à notre denxième sous-genre, et que, comme les Notarches, elle vit uniquement sur les fucus.

\section{A. LEVIS,}

Dolabella lævis, Bunfvirue, Dict. des sc. nat, t. XIII, p. 355; DeshaYes, Dict. class. d'hist. nat., au mot Dolabelle.

Cette espèce établie par MI. de Blainville est ainsi décrite, "animal renflé, à peau lisse. Coquille " non calcaire, mais membraneuse et en forme de "hache."

Cette description étant insuffisante, nous ne savons dans quelle section de notre premier sousgenre nous devons la faire entrer.

M. Deshayes, dans l'article Dolabele du Dictionnaire d'histoire naturelle, la rapporte dans sa synonymie à la Dolabelle fragile de M. de Lamarck; 
nous ignorons sur quoi se trouve fondé ce rapprochement, car nous ne voyons pas quelle analogie de caractère l'autorise. Si d'ailleurs il en étoit ainsi, le $D$. laevis ne se trouveroit être alors autre chose que l' $A$. depilans, puisque la $D$. fragilis n'a été établie que sur une coquille de cette espèce, comme on peut le voir dans la Collection de MI. le duc de Rivoli, où elle est conservée avec la dénomination que lui a imposée M. de Lamarck.

\section{A. RONDELETII.}

Rondelet. De Piscibus, 520.

Dolabella.Rondeletii, Cuvier, Règne anim. t. II, p. 398 .

C'est sans doute la seconde espèce de Lièvre marin de Rondelet; mais si l'on considère bien la figure qu'il en donne, on est tenté de ne pas la rapporter davantage au genre Aplysie qu'au genre Dolabelle, car elle présente bien dans l'ensemble de ses formes l'aspect d'une Aplysie, mais si on l'examine en détail, on n'en retrouve pas tous les caractères; d'abord elle ne montre que deux tentacules, puis on $\mathrm{y}$ cherche vainement quelque chose qui indique la cavité branchiale. Si l'on compare cette figure à celle que Rondelet a donnée de son premier Lièvre marin, et qui ne laisse que bien peu de chose à désirer, on conviendra qu'il est impossible que toutes deux aient été faites pour des espèces du même genre. Nous ne pouvons donc, comme tous les auteurs qui ont parlé de cette espèce avant nous, la considérer qu'avec doute, puisque d'ailleurs elle n'est appuyée sur aucun caractère important.

A. UNICOLOR, Blainvilue, Journ. de phys. 96 , janvier 1823, p. 287 , pl.? f. 9-10; et Dict. des sc, nat, au mot LiÈvre Maris.

Nous croyons reconnoître dans ce mollusque, que nous avons retrouvé dans la collection de M. Brongniart, un jeune individu de l'une de nos espèces de France, et c'est pourquoi nous n'en avons pas fait mention dans le premier groupe de la troisième section du sous-genre Aplysie proprement dite; cependant, comme il se peut que nous soyons dans l'erreur, nous rapporterons ici la description de M. de Blainville, en renvoyant pour la figure à celles qui ont été données dans le Journal de Physique.

"Le corps épais, gibbeux, de couleur uniforme, "blanc-roussâtre; les lobes du manteau entourant "d'une manière assez serrée le bouclier, qui est - plus antérieur que dans les autres espèces, et " dont la coquille est mieux formée, plus large, "plus arrondie, et surtout beaucoup plus bom"bée."

Cette espèce n'a guère que 18 à 20 lignes de longueur. Les individus de la collection de M. Brongniart viennent de Toulon et de Bayonne.

\section{A. UNICOLOR, Risso, J. de phys. t. LXXXVII, p. 374 .}

M. Risso décrit cette espèce darıs le Journal de Pliysique, volume LXXXVII, sous le nom d' $A$. unicolor, mais il n'en parle plus dans son livre des Mollusques de l'Europe Méridionale, ou peutêtre est-ce celle qui est décrite sous le nom. $\mathrm{d}^{\prime} A$. virescente, afin d'éviter le double emploi du premier nom spécifique déja imposé par M. de Blain. ville à une espèce des bords de la Méditerranée. Ce qui nous engageroit à le croire, c'est que les deux descriptions sont à peu de chose près les mêmes. Nous renvoyons donc à la page 66 où nous avons parlé de cette espèce sous le nom $\mathrm{d}$ 'A. virescens que M. Risšo lui donne dans la planche de son ouvrage où elle est représentée; ainsi donc ce naturaliste lui a tour-à-tour imposé trois noms; le premier a été changé à propos, et il nous a été permis de choisir entre les deux derniers. De toutes les espèces décrites par M. Risso, l'A. virescens est la seule, à cause de sa figure, que nous ayons pu introduire dans nos sections; les autres n'étant pas suffisamment connues, comme l'a déja observé M. de Blainville, nous nous contenterons, pour indication seulement, d'en transcrire les phrases comme il les a données.

A. BORDEE DE NOIR, Risso, Journ. de phys., t. LXXXVII, p. 375 ; et Hist. nat. de l'Europe aMérid., t. IV, p. 43.

"Corpore ovato oblongo, nigrescente, nigerrimo "marginato; capile oblongo; testâ ovato-oblongâ, " vitreâ pellucidâ, succineâ.

" Son corps est ovale oblong, d'une couleur noi« râtre, les bords du manteau d'un noir-foncé; les " tentacules sont obscurs; la tête longue, arrondie; "le pied verdâtre. Longueur 0,036 .

"Coquille ovale, oblongue, vitrée, translucide, 
"couleur succin. Séj. Régions des algues. App. "d’un jaune-foncé; les yeux grisàtres; les tentacules "Mars.

"A. ÉTOILÉE, Risso, Ibid.

Corpore rubro, albo nigroque punctato.

"Testa oblonga.

"Corps ovale, allongé, d'un rouge cramoisi, pâle, "orné de petits points noirs, la plupart tachetés de "blanc au milieu; bords du pied couverts de pe" tites taches en forme d'étoiles; tentacules noirâtres, " tête arrondie ; pied d'un gris-clair. Longueur 0,046 .

"Coquille oblongue, translucide. Séj. Sous les cailloux. App. Février, mars.

" $A$. JAUNE, Risso, Ibid.

"Corpore ovato oblongo, luteo; tentaculis supe"rioribus margine griseis.

"Testá oblonga.

"Cette espèce présente un corps ovale oblong, " gris au sommet; la tête comme sinuée en devant; "le pied d'un gris-sale. Longueur 0,040.

"Coquille oblongue, vitrée, translucide. Séj. "Sous les galets. App. Mars, avril."

\section{A. BRONGNIARTII, Blainville, Manuel de ma- lacologie, p. 472.}

Tout ce que nous sarons de cette espèce, qui est peut-être la même que l' $A$. longicauda de MII. Quoy et Gaimard, "c'est qu'elle est allongée, "à queue subulée; qu'elle a les quatre tentacules "longs et grêles; la cavité branchiale subdorsale, " sans opercule ou coquille. *

Cette espèce appartient évidemment à notre sous-genre Notarche, mais nous ne l'y avons pas mentionnée, à cause de l'insuffisance de sa description et de l'incertitude où nous sommes de savoir si ce n'est pas un double emploi de l'espèce indiquée.

\section{OBSERVATION.}

Les Aplysies n'ont point encore montré de dépouilles fossiles: cela provient sans doute, pour les Notarches, de ce qu'elles n'ont pas de test, et pour les Aplysies proprement dites, de ce que cette pièce est toujours fragile; cependant M. Brongniart nous a montré des empreintes de coquilles fort analogues, sur des schistes appartenant à des terrains de transition qui se trouvent à Corcelles (Haute-Saône), à Dillemburg, à Werden sur la Ruhr, et à la Clape près de Digne. M. Brongniart, toujours empressé d'éclairer, par la communication des objets de sa collection, de même que par ses savants conseils, les naturalistes qui travaillent aux progrès de la science, a bien voulu nous permettre d'examiner ces empreintes de coquilles anciennes: elles nous ont présenté plusieurs espèces appartenant évidemment à un seul genre; toutes sont de forme assez aplaties et circulaires, offrant assez bien l'apparence du test de l'A. fasciata; elles sont fortement marquées de stries concentriques ou rayonnantes; mais leur sommet ne présente point précisément la disposition de celui des coquilles d'Aplysies, et il n'y a point d'échancrure au còté droit; les caractères de ces fossiles qui paroissent d'ailleurs avoir̂̀été de nature très-flexible, présentent trop confusément ceux qui distinguent les Aplysies pour que nous osions en ce moment les rapporter à ce genre. 


\section{gevre deUxième. BURSATELLE, BURSATELLA, Blainville.}

Synonymie. Bursatella, Blainville, Dict. des Sc. nat., et Man. de Malacol.

Corpus repens, subrotundum; suprà convexum, infrà planum; anticè breve, posticè obtusum; cavitas branchiarum minima, operculo privata; margines membranarum angustas, ad natandumque non aptas; pecten branchiarum longissimus atque ad exteriorem cavitatis ejectus; tentacula quatuor, ramosa; appendices duas prater labia.

Descriptron. « Le corps est subglobuleux, dit M. de Blainville, offrant inférieurement un " espace ovalaire circonscrit par des lèvres épaisses indiquant le pied, supérieurement une "fente ovalaire à bords épais, symétrique, formée par la réunion complète des appendices " natatoires du manteau, et communiquant dans une cavité où se trouve une très-grande "branchie libre et l'anus; quatre tentacules fendus, ramifiés, outre deux appendices buc" caux.»

"Aucune trace de coquille.»

Historique. Le genre Bursatelle a été établi par M. de Blainville pour une espèce qui vient des mers de l'Inde, et qu'il a décrite et figurée dans le Dictionnaire des Sciences naturelles. Depuis, aucun auteur n'en a parlé, si ce n'est cependant, comme nous avons déja eu occasion de le dire, M. Audouin, qui, dans l'explication sommaire des planches de Mollusques de la Description d'Égypte, a cru voir dans l'un de ces animaux une Bursatelle, et l'a en conséquence nommé $B$. savignana. MI. Audouin, frappé de la grande analogie qui existe entre les Bursatelles et les Aplysies, a fait des premières un sousgenre de celles-ci. La connoissance que nous avons eue, à l'état de vie, d'une espèce extrêmement voisine de la $B$. savignana de M. Audouin, et que nous avons dédiée à la mémoire de M. Plée, nous a donné lieu de reconnoître que ces deux espèces ne devoient point en effet être séparées des Aplysies, et qu'elles entroient de droit dans notre sousgenre Notarche emprunté à M. Cuvier. Nous aurions peut-être dû y réunir aussi le genre Bursatelle, qui certainement ne doit différer des $A$. savignana et Pleii que par des caractères spécifiques; mais nous n'avons pas osé le faire, n'étant pas suffisamment éclairé sur ce mollusque.

Un motif nous a surtout arrêté dans cette circonstance; il tient à un caractère que M. de Blainville n'a pas reproduit, il est vrai, dans son Manuel de Malacologie, mais qu'il a fait connoître précédemment dans le Dictionnaire des sciences naturelles, et que l'on peut retrouver dans la figure de la Bursatelle qui existe dans l'un et dans l'autre de ces deux ouvrages. Ce caractère consiste dans la présence, "sur le milieu de la tête, d'un organe tentaculaire pouvant rentrer dans une cavité creusée à sa base. » Nous ignorons pourquoi ce savant semble avoir abandonné ce caractère, dont il ne parle plus; mais nous pensons que dans un pareil état d'incertitude, il convient mieux, comme nous l'avons fait, de laisser ce mollusque isolé et en évidence, afin de provoquer à son sujet de nouvelles recherches de la part des naturalistes. 
M. de Blainville, qui ne connoît la Bursatelle que par l'individu conservé au Muséum Britannique, ne nous apprend rien par conséquent touchant ses mours et ses propriétés. Cependant nous pouvons penser que vu leur grande analogie avec les mollusques de notre sous-genre Notarche, elles ne doivent point différer avec eux sous ce rapport.

\section{ESPËCE UNIQUE.}

BURSATELLA LEACHII, Buarvilue. Dict.des à M. de Blainville : "La Bursatella Leachï est $S c . n a t$., To. V; et Man. de malacol., p. 473 , "presque grosse comme le poing, d'une coupl. XLIII, f. 6; Férussac, Dict. class. d'hist. "leur d'un blanc-jaunâtre, comme translucide; nat. To. II, p. 588.

" tout son corps est parsemé de petits appendices

"tentaculiformes, irrégulièrement disposés : ce

Corpore globuloso, albido-luteo, substranslucido, appendicibus tentaculiformis irregulariter cooperto.

Description. La description de cette espèce se
orne à ce peu de mots que nous empruntons "qu'on nomme peut-être à tort tentacule dans " cette famille, et les bords antérieurs de la tête en " ont de plus longs."

Hábitation. M. de Blainville dit qu'elle vient des mers de l'Inde. 


\title{
B. Deux tentacules; les yeux̀ situés à leur base et en arrière.
}

genré troisième. A $\mathrm{C}$ TÉ $\mathrm{ON}, A C t \not e n$, Ocken.

\begin{abstract}
Synomyme. Laplysia, Montagu; Actceon, Ocken; Férussac.
\end{abstract}
Genre Elysia? Risso.

Corpus repens, elongatum, posticè acuminatum; membranoe laterales duas ad natandum aptce; tentacula duo; oculi sessiles posticè, ad basim tentaculorum; testẩ privatum.

Description. L'Actéon a le corps allongé, limaciforme, acuminé postérieurement; le manteau offre latéralement deux expansions membraneuses analogues à celle de la troisième section des Aplysies proprement dites et pouvant servir à la natation; en avant, le mollusque est muni de deux tentacules cylindriques, obtus et assez gros; les yeux sont situés à la base et en arrière de ces organes; point de rudiment testacé interne; branchies et organes de la génération inconnus.

Historique. Montagu a fait connôitre, le premier, le mollusque qui depuis a servi à l'établissement du genre Actéon; il en traite assez longuement dans le tome VIII des Transactions Linnéennes. Remarquant l'analogie que ce petit animal montre avec les Aplysies, il crut en avoir découvert une espèce nouvelle, et le décrivit sous le nom de Laplysia viridis. Malheureusement cette description laisse beaucoup à désirer; par exemple, il ne parle point des branchies, et ne nous apprend pas' sil existe dans ce mollusque comme dans les Aplysies une cavité dorsale protégée par un opercule; seulement il dit qu'il n'y a point de rudiment testacé, ce qui est bien loin de suffire actuellement pour nous éclairer à ce sujet. Montagu ne dit rien non plus de la position des organes de la génération et de l'anus, caractères bien propres cependant à déterminer le genre auquel doit appartenir cette espèce. Depuis, Ocken, sur la description et la figure de Montagu, a cru reconnoître dans la Laplysia viridis un mollusque tellement différent de ce que croyoit Montagu, qu'il le rapporta aux Pulmonés, fixant sa place entre l'Onchidie de Buchannan et le genre Limace. Sans doute ce savant se laissa entraîner à ce rapprochement par certains rapports d'ensemble et de forme qui paroissent exister entre ce mollusque et les Limaces, car aucun caractère plus important ne put certainement l'y autoriser; aussi M. de Férussac adopta bien, il est vrai, le genre Actéon d'Ocken, mais il repoussa, comme tout autre naturaliste l'auroit fait, sans doute, la désignation d'une place parmi les Pulmonés.

Ce savant, dans ses Tableaux systématiques, de même que dans le premier volume du Dictionnaire classique d'Histoire naturelle, rétablit ce mollusque dans la place que Montagu lui avoit assignée en le mettant dans l'ordre des Tectibranches, famille des Discènes, qui est la même que celle des Aplysiens, et tout à côté des Aplysies mèmes. Nous ne changerons rien à ce que M. de Férussac a fait au sujet du genre Actéon, mais nous n'y apporterous pas comme lui l' $A$. viridis de M. Bosc, que nous avons jugé plus à propos de 
faire entrer dans le sous-genre Notarche, parce que nous avons cru y reconnoître deux paires de tentacules.

Nous ajouterons à ces observations sur un genre si mal connu, que $\mathbf{M}$. Risso, dans le tome IV de l'Histoire naturelle de l'Europe Méridionale, décrit sous le nom d'Élysie un genre de mollusque qui paroît être le même que le genre Actéon, si toutefois on examine la figure seulement, car la description porte des caractères tellement extraordinaires, que l'on seroit tenté, non-seulement de refuser ce rapprochement, mais même de rejeter le genre Élysie, ou mieux de le renvoyer à un nouvel examen de son auteur.

\section{ESPËGE UNIQUE.}

ACTEON VIRIDIS, OCKEN. Pl. XXIII, f. 9 .

Laplysia viridis, Montagu, Trans. linn., t. VII, p. $76, \mathrm{t} . \mathrm{VII}, \mathrm{f} . \mathrm{x}$.

Actæon viridis, Oćк EN, Lehrbuch der zool., t. II, p. 305 ; Fénussac, Tab. syst. p. xxx, et Dict. class. dhist. nat., t. I, p. го4.

Elysia timida? Rrsso, Hist. nat. de l'Europ. mérid., t. IV, p. 45, Journ. de Phys., 87. Blainville, Man. de malacol., p. 474.

Corpore elongato, in medio convexo, supernè viride, subtus luteo; posticè elevato, carinato; membranis lateralibus elongatis, angulatis.
Description. Elle ressemble beaucoup à unt Limace; elle est toute verte; ses flancs, vers le pied, sont jaunâtres; sa tête est courte; son corps est acuminé et élevé postérieurement en forme de carène; sa partie médiane est convexe, couverte d'une membrane élargie en forme d'ailes ou de nageoires, et s'étendant en arrière en diminuant de largeur et bordant la carène. Elle prodnit une liqueur purpurine comme les Aplysies. Long. 0,028. Habitation. Les côtes du Devonshire en Angleterre.

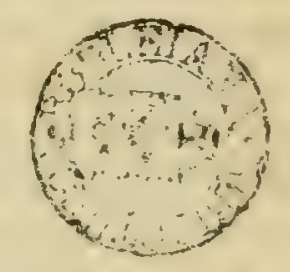

FIN. 


\section{EXPLICATION DES PLANCHES.}

\section{PLANCHE I.}

A. Rumphri l'animal vu en dessus et du côté droit; la coquille de l'opercule; la position de la cóquille par rapport à la fente dorsale et à la partie súpérieure du disque.

\section{PLANCHE II}

A. ECAdDdata; l'animal vu en dessus et du cóté droit; sa coquille vue en dessus ainsi que de profil.

\section{PLANCHE III.}

A. Teremidi; fig. $x$, l'animal vu en dessus; fig. 2, sa coquille vue en dessus; fig. 3 , la même vue de profil.

A. Grgas; fig. 4, sa coquille vue du côté infériẹtr.

\section{PLANCHE IV.}

A. dolabrifera; fig. I, l'animal vu du côté droit, et pendant sa marche; fig. 2 , sa coquille vue en dessous; fiğ. 3 , le même vu de profil ; fig. 4 , l’animal contracté; fig. 5 et 6 , coquille monstrueuse représentée en dessous et de profil.

A. Ascifera; fig. 7, sa coquille en dessous; fig. 8, la même de profil; fig. 9, l'animal du côté droit.

\section{PLANCHE V.}

A. petaltfera; fig. I, l'animal vu en dessus; fig، 2 , la coquille de profil ; fig. 3 , la même en dessous.

A. uxguifera; fig. 4, l'animal vu en dessous; fig. 5 , le même par côté; fig. 6 , la coquille de profil ; fig. 7 , la même en dessous.

\section{PLANCHE VI.}

A. Fasciata; la figure ombrée représente l'animal vu du côté droit et nageant à l'aide de ses lobes latéraux; la figure au trait montre la disposition de l'opercule, du syphon et du panache des branchies de mẻme que celle des lobes. (C'est par erreur que l'on a fait une petite ouverture à la membrane, il fallait un petit tube comme on le voit dans la fig. I de la pl. virr.) La coquille est représentée de profil et du côté inférieur.

\section{PLANCHE VII.}

La même espèce; fig. I, deux individus au moment de l'accouplement; fig, 2 , la verge en partie sortie de sa gaîne, et le même organe tout entier en dehors; fiğ. 3 , une pelote d'oeufs de grandeur naturelle; fig. 4, plusieurs détails des oeufs grossis.

\section{PLANCHE VIII.}

La même espèce; coquilles monstrueuses; fig. 4 , coquille ayant à sa face inférieure une cloison verticale et transverse; fig: 5 , coquille ayant le sommet conique et situé près de son centre à la face supérieure; fig. 6, la même, vue de profil; fig. 7, la même représentée en dessous pour montrer sa cloison horizontale.

A. Brasiliana; fig. r, l'animal vu en dessus, et les lobes rabattus pour nager; fig. 2 , la coquille du côté inférieur; fig. 3 , la même de profil.

\section{PLANCHE IX}

A. Dactylonela ; l'animal est viu de cóté et en dessus, les lobes étant seulement un peu écartés; la coquille est vue en dessous et en profil.

\section{PLANCHE X.}

A. Protea; fig. I, l'animal vu de côté, les: lobes'renversés sur le dos; fig. 2 , sa coquille de profil; fig. 3 , la même en dessous.

A. Sorex; fig. 4, l'animal vu de côté; fig. 5, vu eì dessus; fig. 6 , vu en dessous pour montrer le pied; fig. 7 , sa coquille représentée du côté inférieur; fig. 8, la même de profil.

\section{PLANCHE XI.}

A. ticrtiva; l'animal est vu du côté droit, les lobes croisés sur le dos, et en dessus les lobes rabattus pour nager; la coquille est représentée en dessous et de profil.

\section{PLA NCHE XII.}

A. Maculata; fig. I, l'animal vu de côté, les lobes croịsés; fig. 2 , le même vu en dessus, les lobes séparés; fig. 3 , la tête vue de face pour montrer la disposition des tentacules labiaux chez toutes les espèces de la même section; fig. 4 , sa coquille vue en dessous; fig. 5 , la même vue de profil.

A. marmorata; fig. 6, l'animal vu en dessus, les lobes rabattus pour nager; fig. 7 , le même vu de côté, les lobes croisés; fig. 8 , sa coquille de profil; fig. 9 , la mème en dessous. 


\section{PLANCHE XIII.}

A. Íreraudrenir; fig. I, l'animal vu en dessus et développé comme lorsqu'il nage; fig. 2 , le même vu en dessous et un peu contracté; fig. 3 , la coquille en dessous; fig. 4, la même de profil.

\section{PLANCHE XIV.}

A. Lessonir; l'animal est représenté jar deux figures; dans l'une il est vu de côté et un peu en dessous, dans l'autre il est vu en dessus; les lobes rabattus de chaque côté pour montrer la disposition de l'appareil branchial; la coquille est vue en dessous et de profil.

\section{PLANCHE XV.}

A. Camelus; fig. I, l'animal vu en dessus, les lobes rabattus de chaque côté.

A. ALBa; fig. 2, vue en dessus; fig. 3 , de côté. Les figures de cette planche sont copiées sur celles que M. Cuvier a données dans les Annales du Muséum.

\section{PLANCHE XV bis.}

A. zizapolitana; fig. $\mathbf{x}$, l'animal vu en dessus, les lobes très-écartés; l'opercule soulevé; le syphon trèsouvert.

A. poliana; fig. 2, l'animal vu en dessus, les lobes écartẻs; le panache des branchies flottant au dehors, et la verge sortie de sa cavité. Les figures de cette planche sont copiées sur celles publiées par M. delle Chiaje dans un mémoire intitulé, Memorie sulla Hist. nat. e notom. delle Aplysie.

\section{PLA NCHE XVI.}

A. oepilans; fig. I, l'animal vu du côté droit et les lobes redressés; fig. 2 , le mème très-contracté; fig. 3 , disposition des tentacules labiaux; fig. 4 , position des yeux et forme des tentacules supérieurs.

\section{PLANCHE XVII.}

La même; fig. I, l'animal vu en dessus, les lobes écartés pour montrer les caractères de l'operçule; fig. 2 , la coquille en dessous; fig. 3 , le même de profil; fig. 4 , l'animal au trait vu de face pour montrer l'ouverture formée en avant par le rapprochement des lobes.

\section{PLANCHE XVIII.}

A. rusca; fig. I, l'animal très-contracté. Cette figure est copiée de celle qque M. Tilésius a donnée dans le Voyage de Krusenstern.

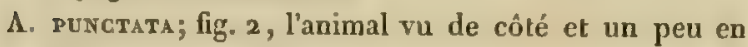
dessus pour montrer l'ouverture de l'opercule; fig. 3 , la coqquille en dessous; fig. 4 , la même de profil.

\section{PLANCHE XIX.}

A. xongicorsis; fig. I, l'animal de côté; fig. 2 , en dessus; fig. 3 , la coquille en dessous; fig. 4 , de profil.
A. Ferussacir; fig. 6, l'animal vú de côté; fig. 7 , en dessus; fig. 8 , la coquille en dessous; fig. 9 , de profil.

A. virescens; fig. 5 , vue en dessus. Cette figure est empruntée à l'Histoire des Mollusques de l'Europe Méridionale, par M. Risso.

\section{PLANCHE XX.}

A. SavigNana; fig. I, individu de grandeur naturelle vu en dessus; fiğ. 2 , le même vu en dessous; fig. 3 , le même vu en profil et dı côté droit; fig. 4 , un tentacule postérieur, montrant l'ouverture formée par un repli; fig. 5, le peigne branchial un peu grossi, vu en dessus et près du cœur; fig. 6 , le même vu dans son épaisseur et ses deux faces; cette dernière figure montre parfaitement de quelle manière les palettes branchiales sont adaptées de chaque côté d'un diaphragme; fig. 7 , la tête vue du côté de la bouche; fig. 8 , partie intérieure de la bouche; fig. 9 , to et II représentent sous différentes faces la pièce armée de crochets qui servent d̀ la mastication; fig. 12 et $\mathbf{r} 3$, ces mêmes crochets considérablement grossis.

\section{PLANCHE XXI.}

A. PleiI; fig. I, l'animal vu du côté droit; fig. 2, vu en dessus, la cavité dorsale un peu ouverte; fig. 3 , vu en dessous; fig. 4, le peigne branchial.

\section{PLANCHE XXII.}

A. ctrrina; fig. I, vue en dessus; tig. 2 , vue du côté gauche.

A. nudata; fig. 3, vue en dessus; fig. 4, vue du côté gauche; fig. 5 , une pelote des oufs de cette espèce.

A. Limacina; fig. 6 , vue en dessus; fig. 7 , vue du côté gauche.

A. Loxcicauda; fig. 8, vue en dessus. Cette figure est copiée de celle que MIM. Quoy et Gaimard ont donnée dans l'atlas du Voyage de l'Uranie. Fig. 9, la même vue de profil et un peu en dessus; cette figure nous a été communiquée par M. Godichand qui l'avait faite à borl de l'Uranie sur l'animal vivant; il y a joint la figure so pour montrer la disposition des tentacules.

A. VIripis; fig. II, vue en dessus.

\section{PLANCHE XXIII}

A. Rosea, fig. 6, l'animal vu du côté droit et la verge hors de sa cavité; fig 7 , sa coquille. Ces deux figures sont copiées des Mémoires de la Société d'Hist. nat. de Copenhague.

A. Gelatinosa (c'est par erreur que l'on a mis Gelatinosus); fig. I, l'animal vu en dessus; fig. 2, vu du côté droit et les branchies flottant en dehors; fig. 3 , vu en dessous pour montrer le pied tel qu'il est lorsqu'il rapproche ses bords pour saisir les plantes; 
fig. 4 , le peigne branchial; fig. 5 , le pied ouvert dans toute sa longueur.

Bursatella Leachi; la fig. 8 qui représente l'animal vu en dessus et portant les branchies à l'extérieur, est copiée de M. de Blainville dans son Traité de Malacologie.

Actron viridis; fig. 9, l'animal est vu en dessus et un peu de cồté. Cette figure est empruntée aux Mémoires de la Société Linnéenne de Londres.

\section{PLANCHE XXIV.}

Aplysia HasseltiI; fig. I, l'animal est représenté en dessus. Cette figure ainsi que les suivantes sont empruntées aux belles planches que M. Van-Hasselt avait fait faire à Java et qui ont été sỉ généreusement communiquées à M. de Férussac par M. Temminck.

Aplysia ocellata; fig. 2, vue en dessus; fig. 3, vue du côté droit; fig. 4, la tête vue de face. 


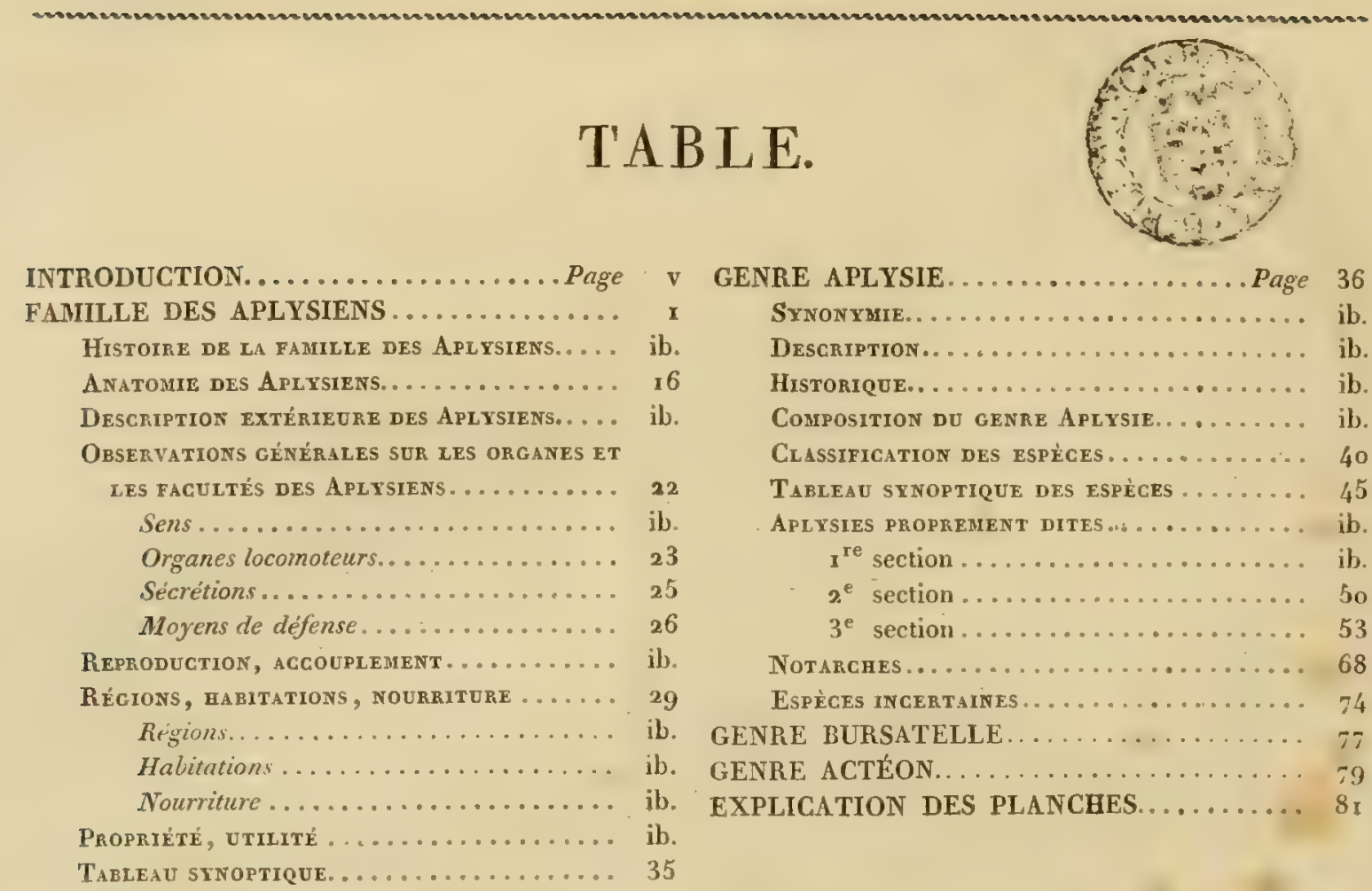




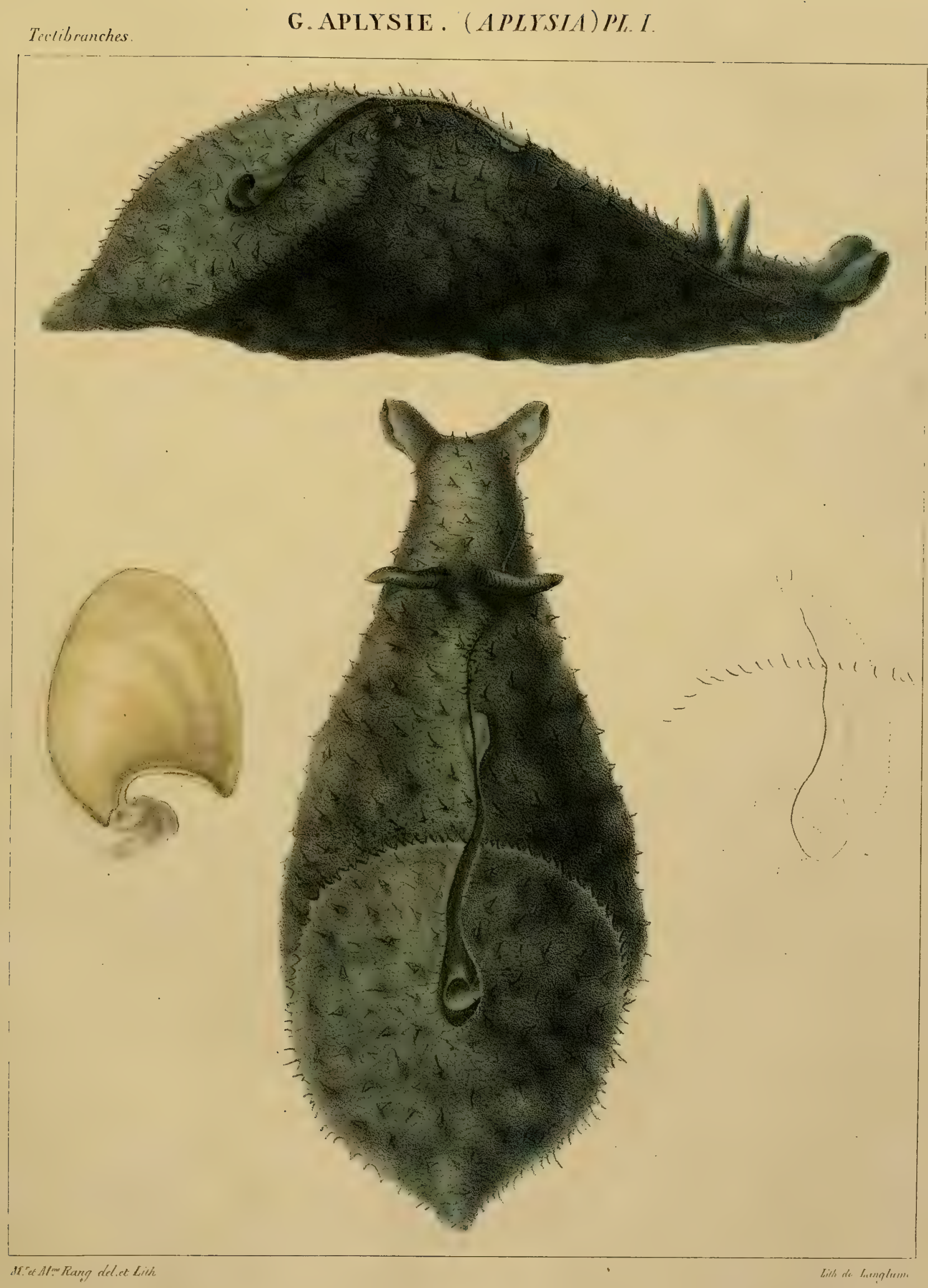

1. (s.c.opplyssiss preprement dirss) Rumphii. 
(.) 

Tectibranches.
G. APLYSIE. (APLYSTA) PLIII

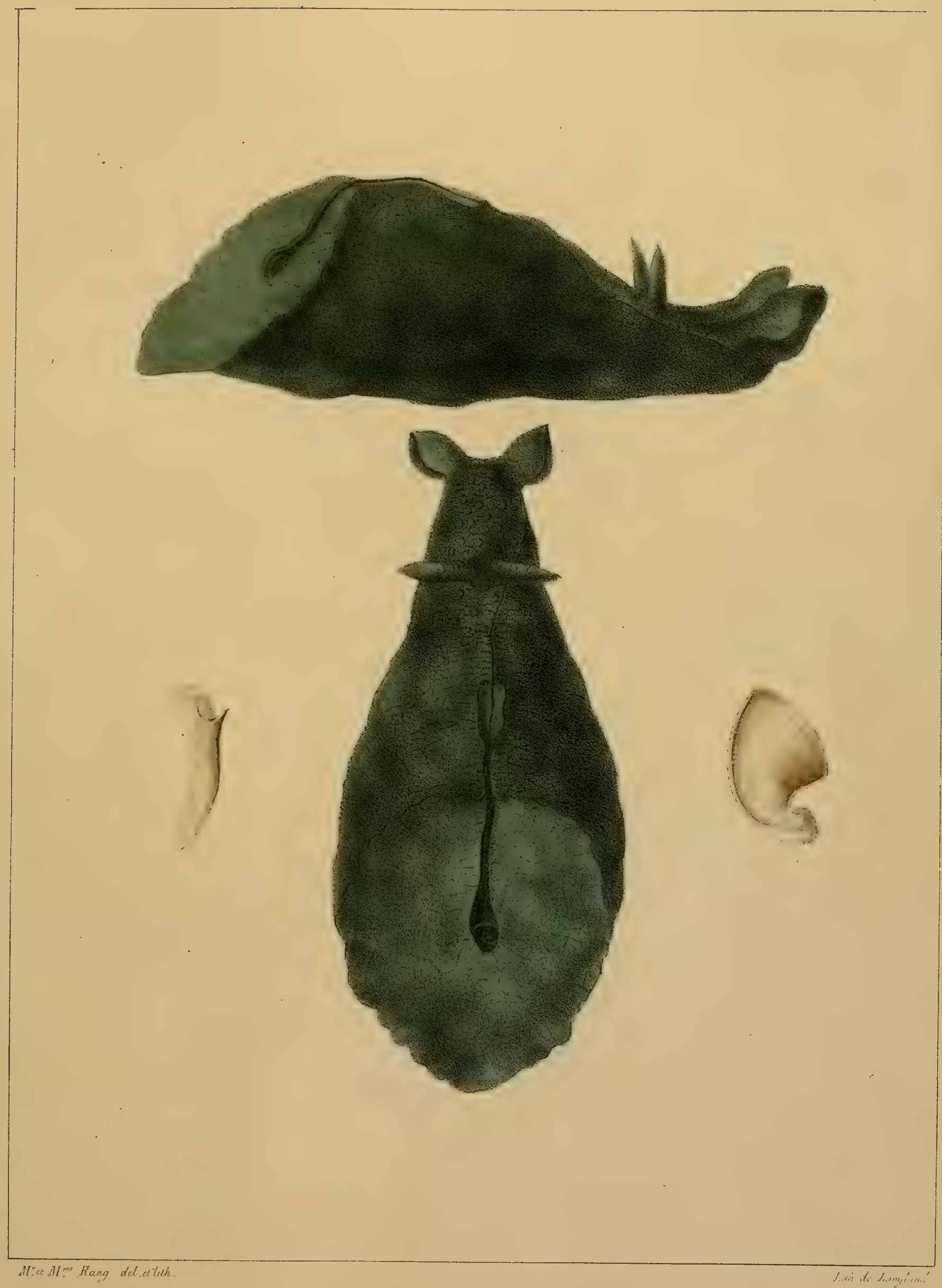

A. (S.G. Uplysies proprement diles) Ecaudata, Rany. 



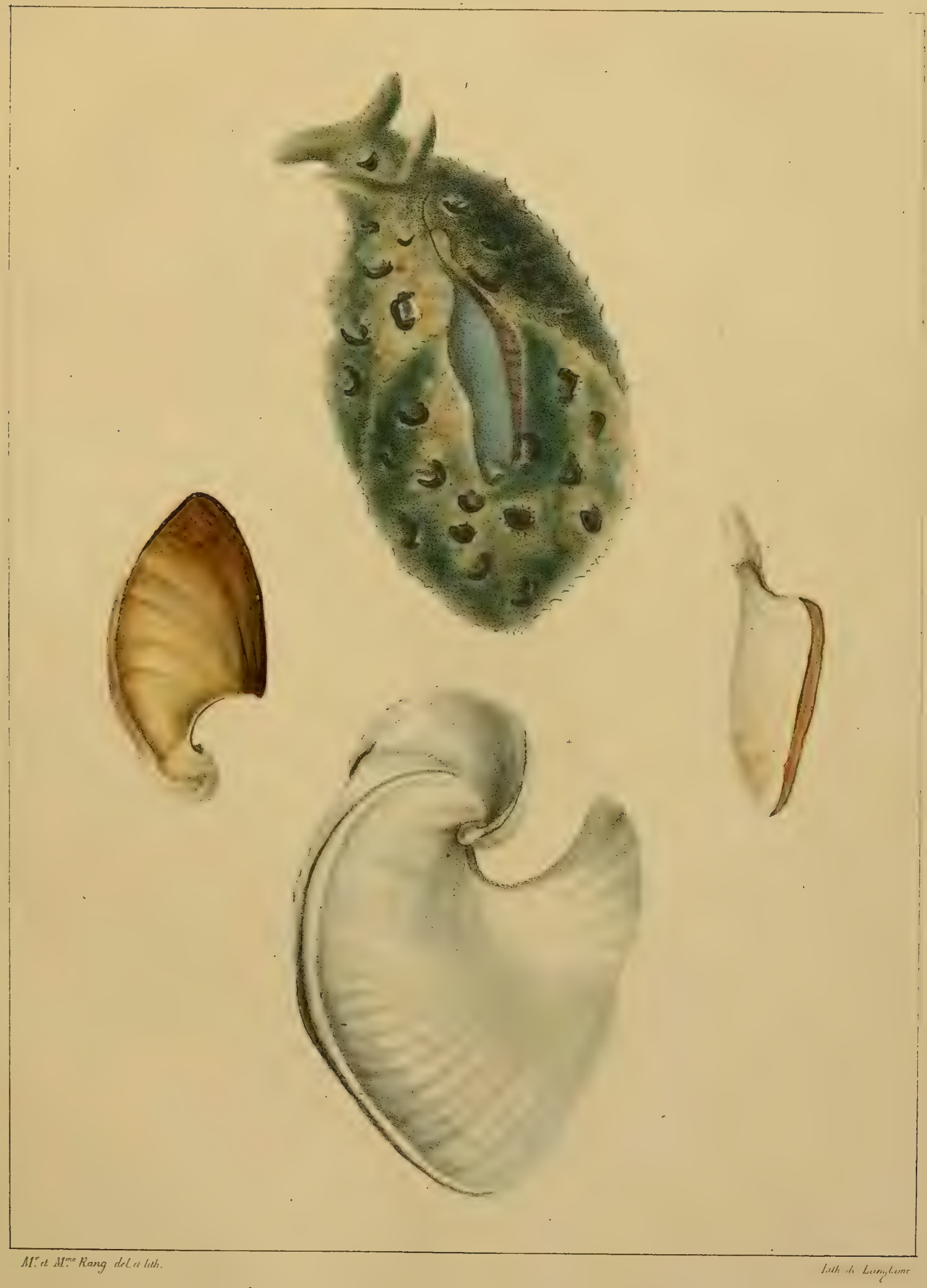

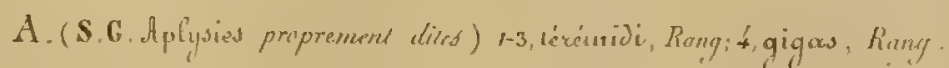


C. 


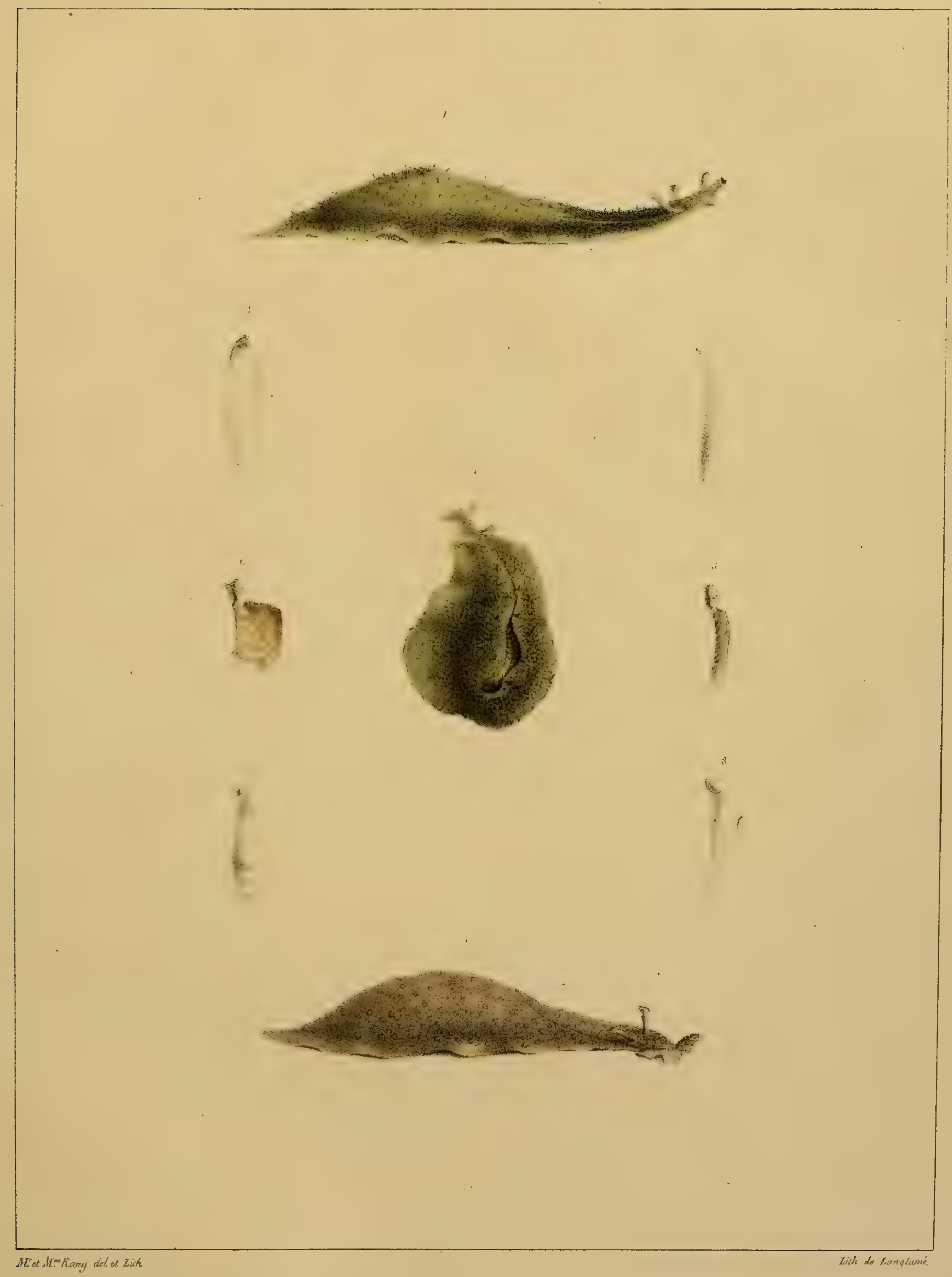

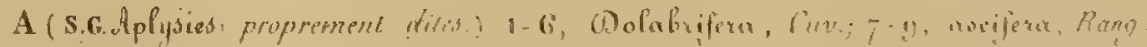


c. 


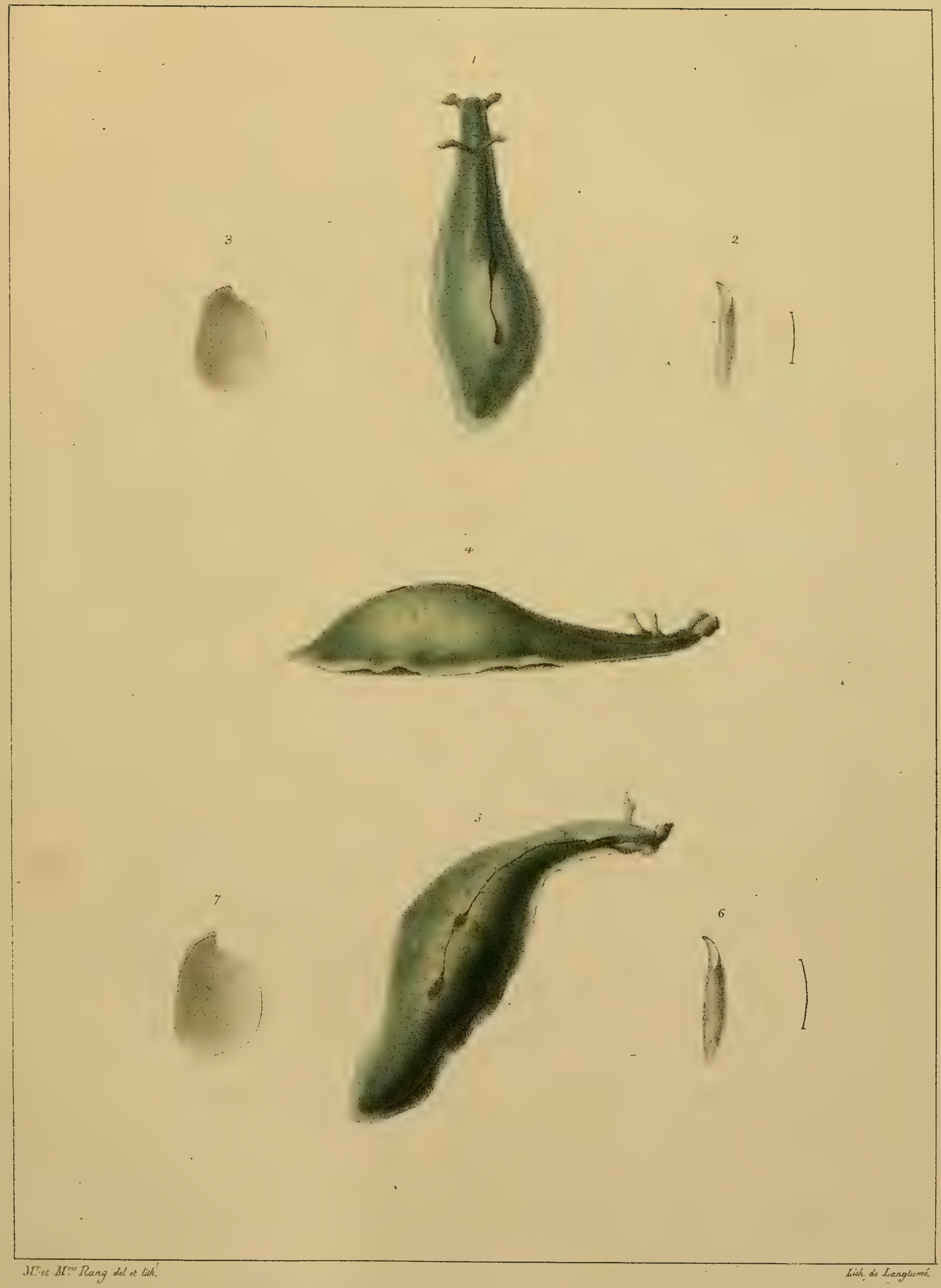

A. (S.G. Aloplysies proprement dites.) 7-3, Petalifeco, Rang; 4-7, iunguifera, Rang. 
(9) 


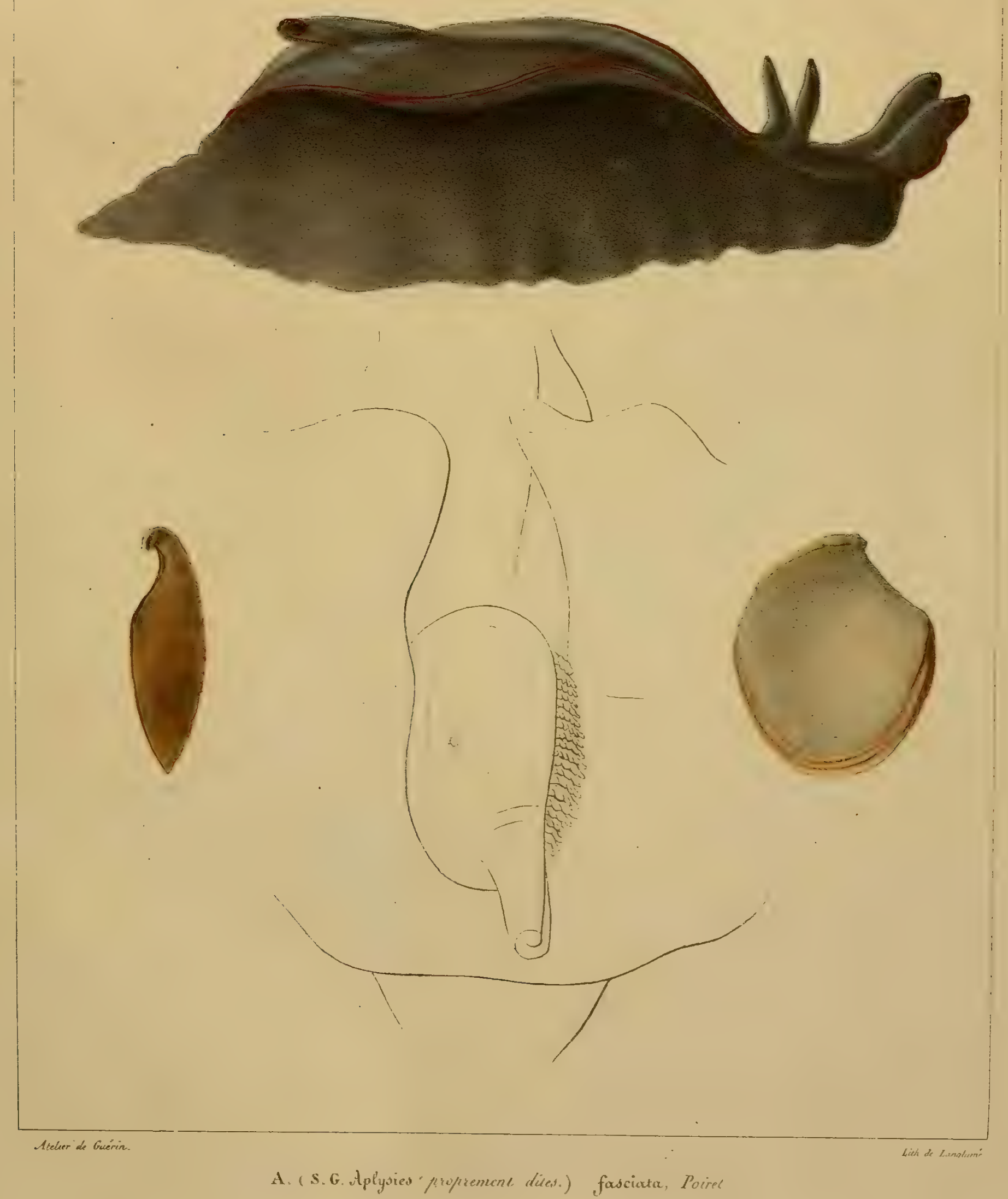





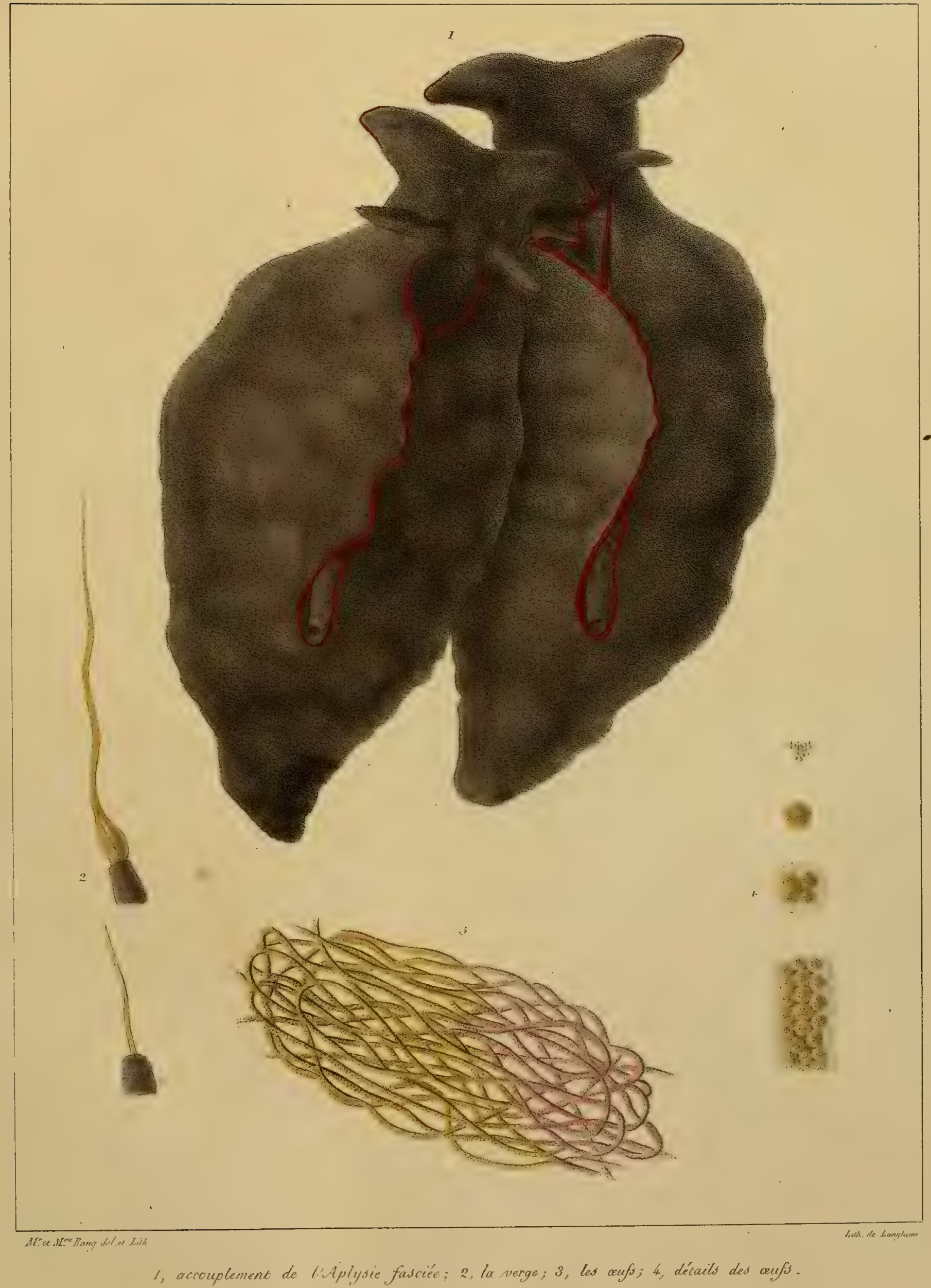





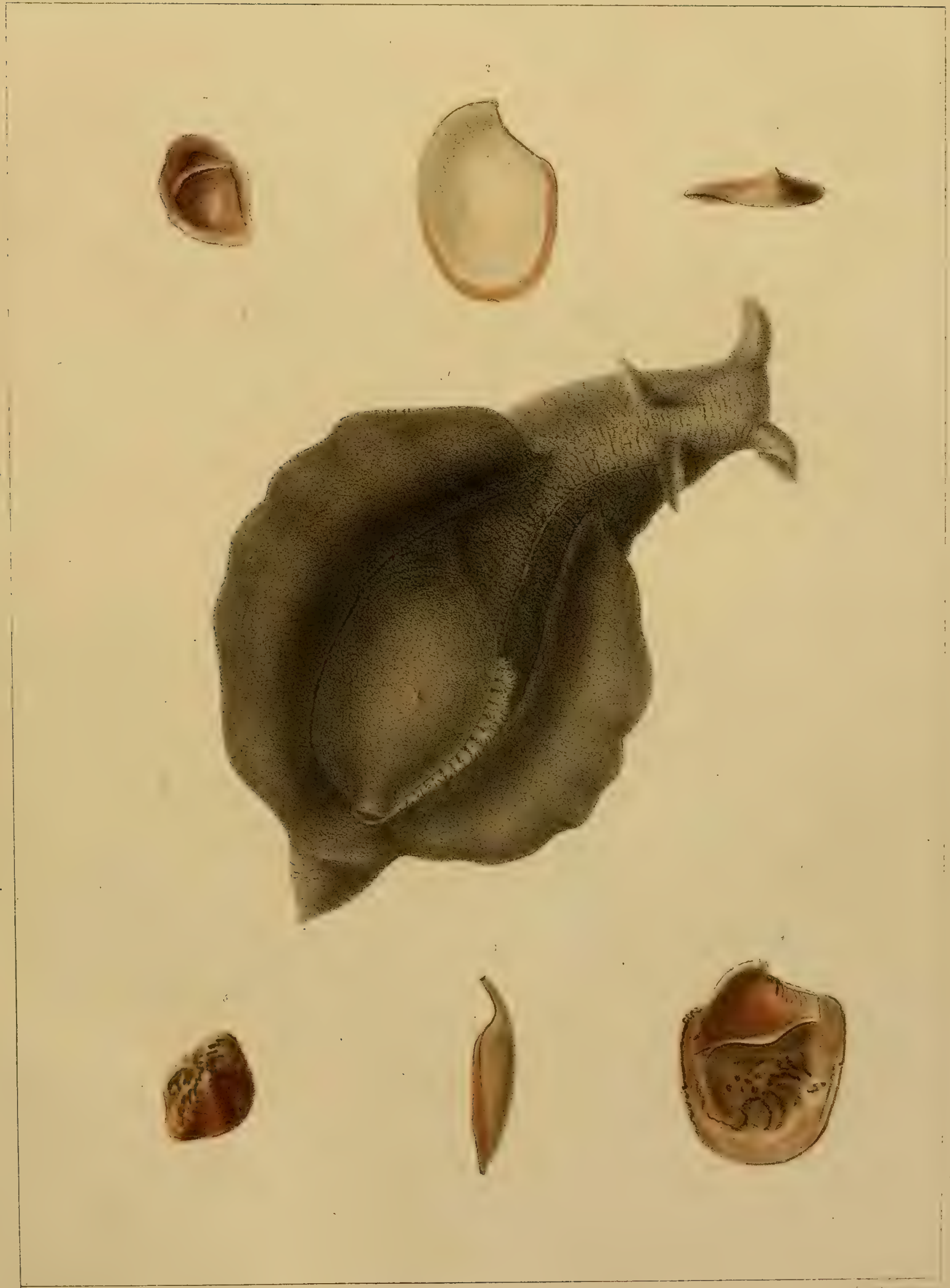

Mret Mne Rang deder lith.

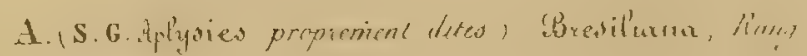




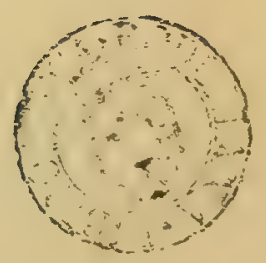


Tecribranches. $\quad$ : G. APLYSIE. (APLYSIA.) PII. IX

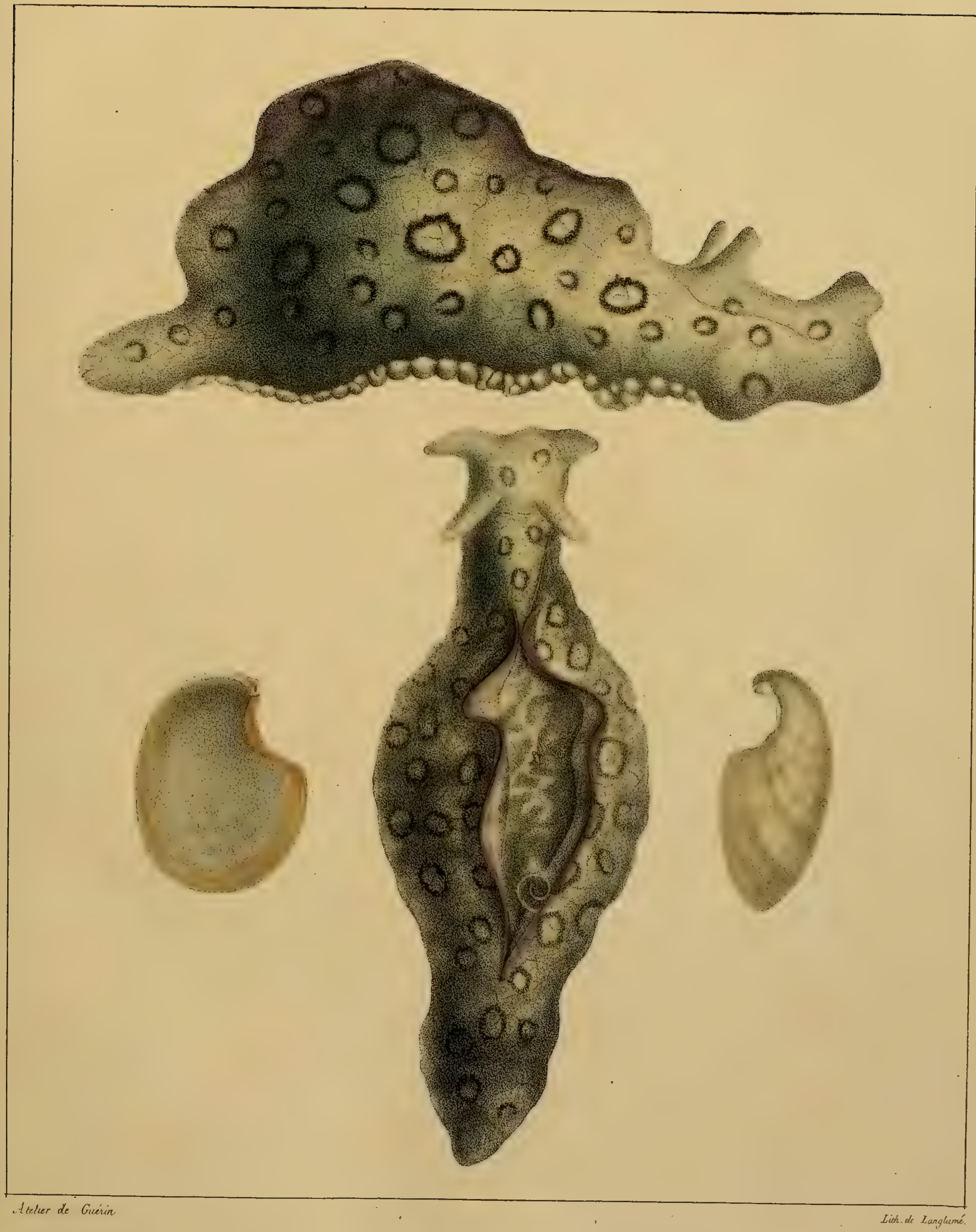

A. (S. G. Applyoies proprement dies.) Jactylomelö, Rang. 
. 


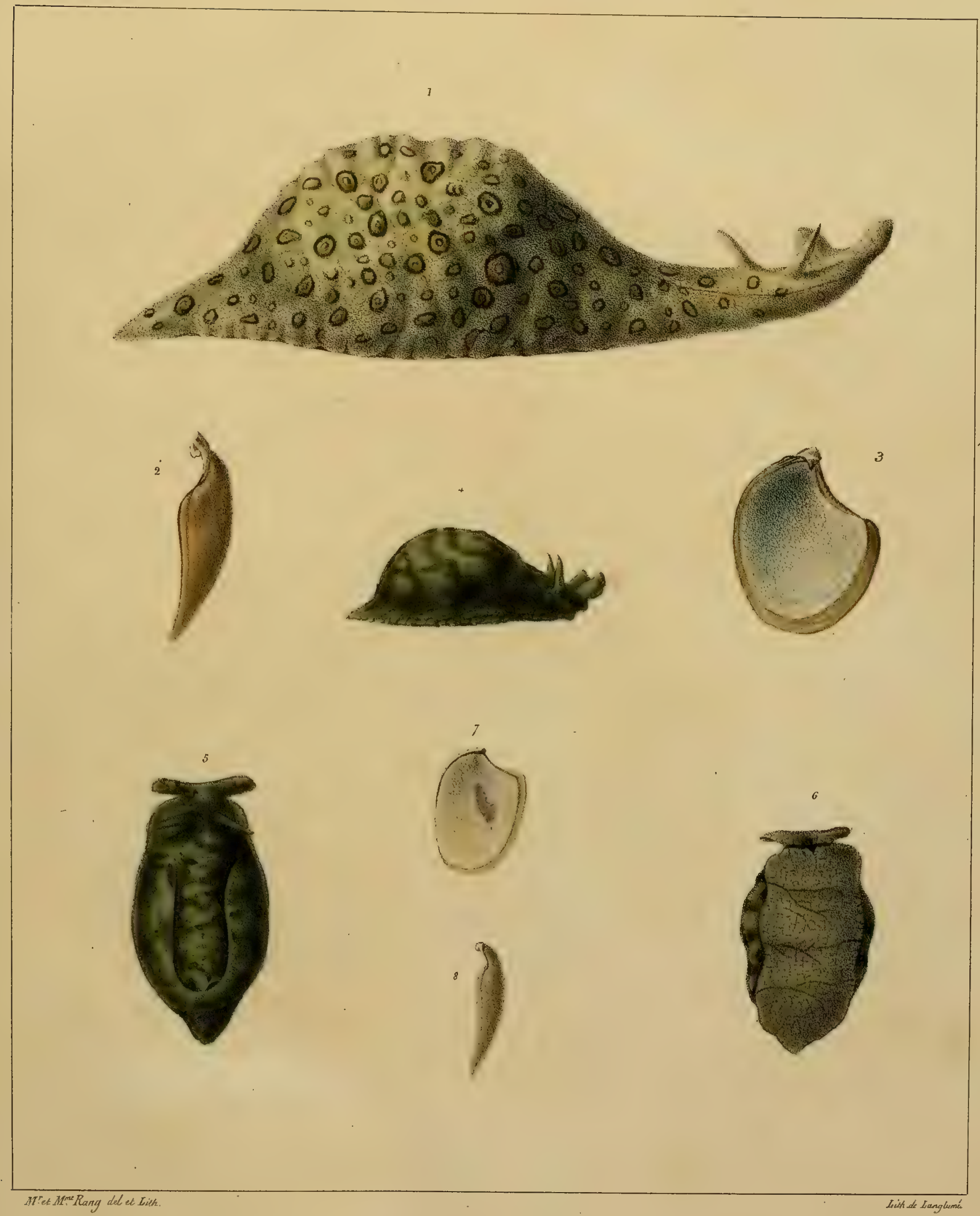

A. ( S.G. Aplysies proprement dites.) 1-3, Protex, Rang; 4-8, Sorex, Rang. 



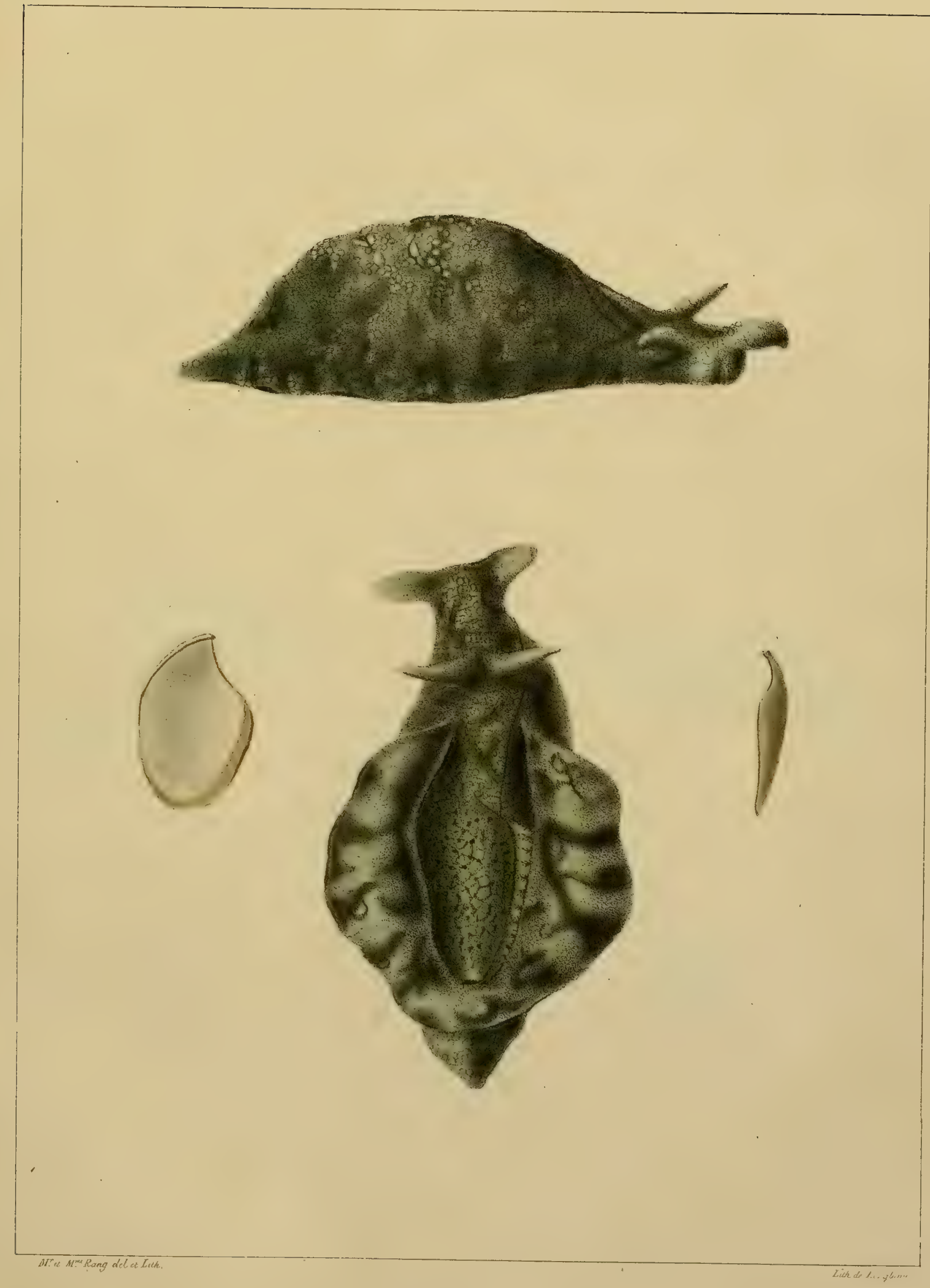

A. ( S.G.Aplysies propuement-diero.) tigrsitow, Rang. 



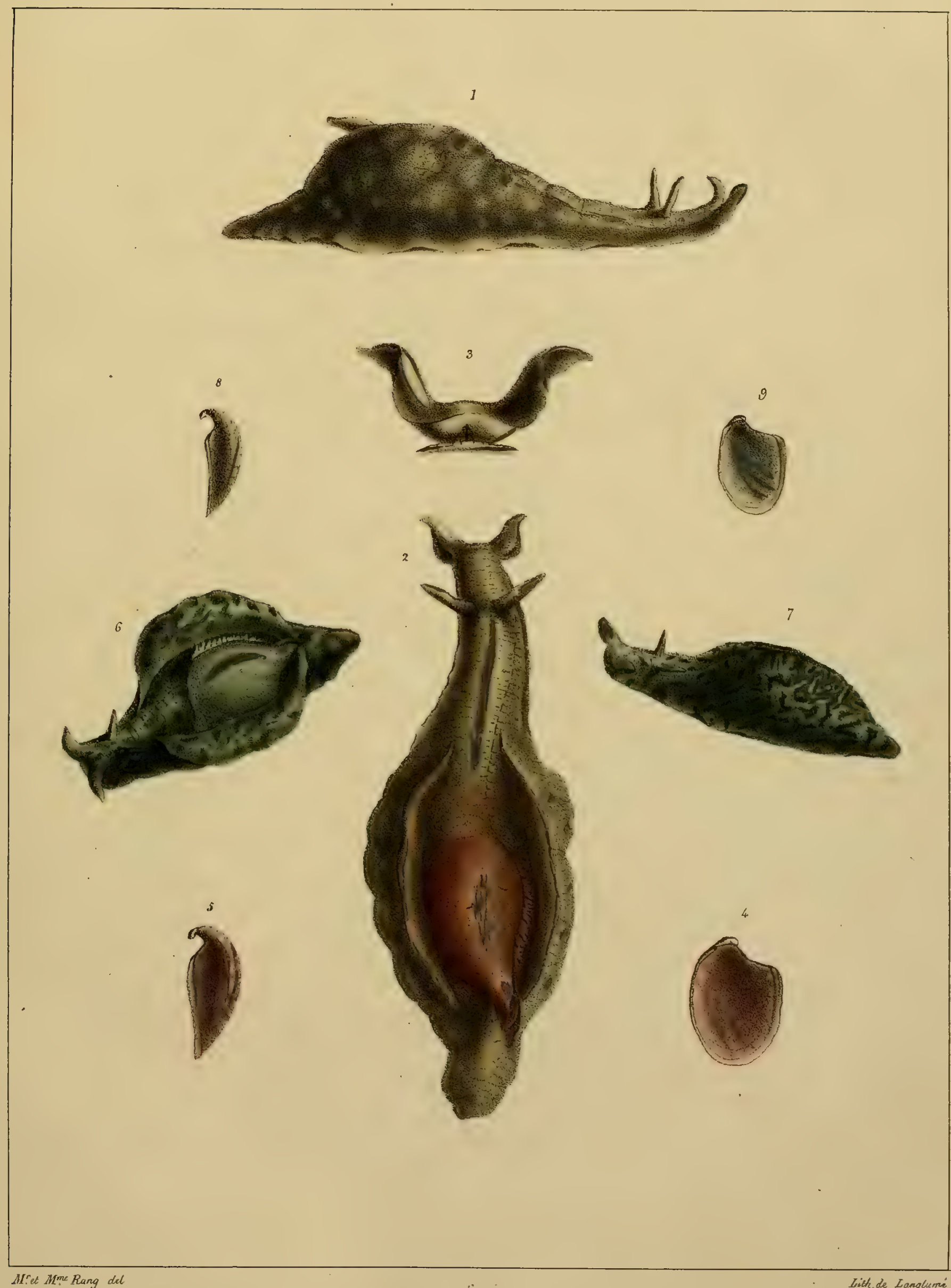

A. ( S.G. Aplyoies proprement dites.) I-5, miaculatox Rang; 6-9, marnorato, Blainville. 
(1) 


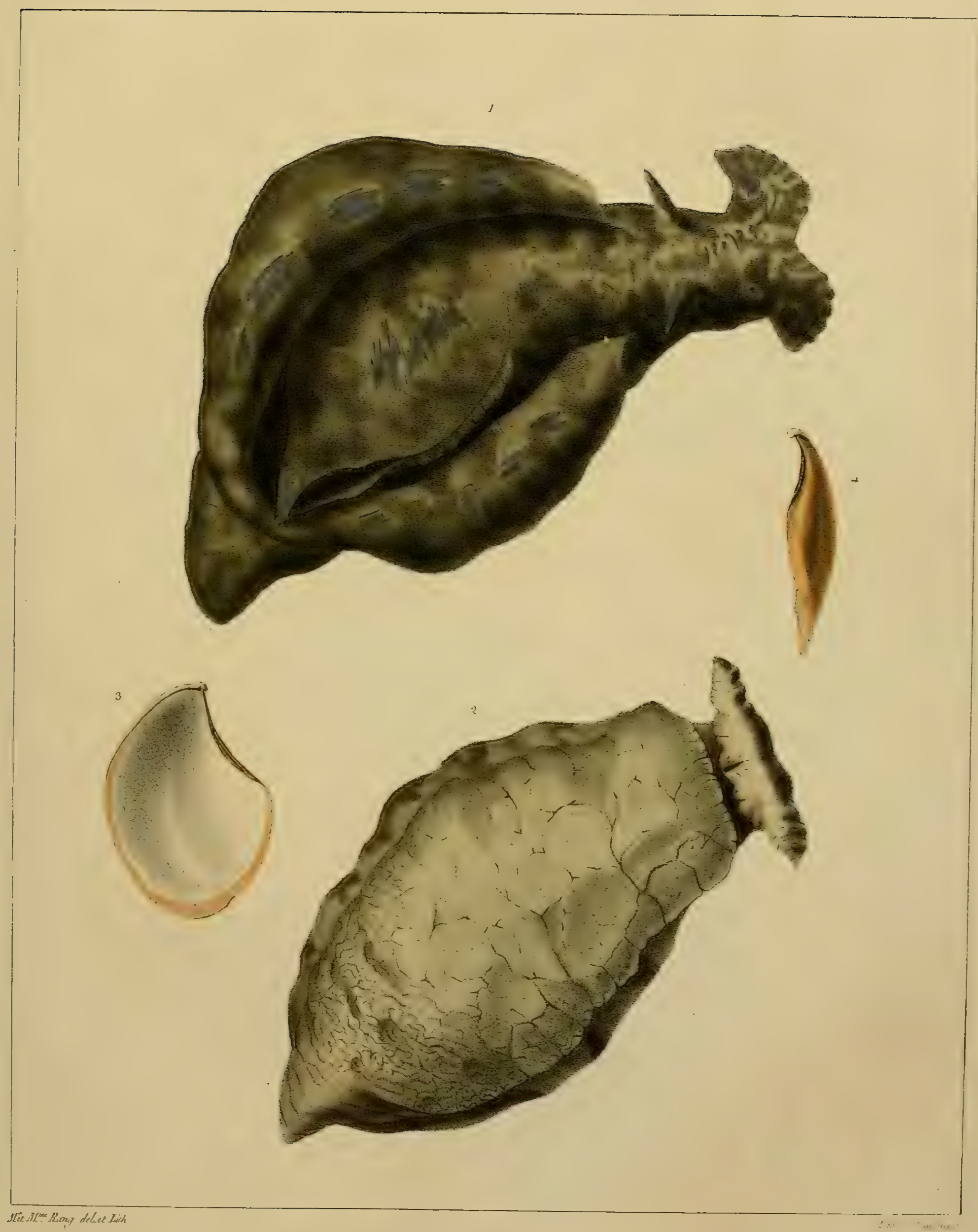

A. (S.G. Aplyjies proprement dites.) 1-4, Kerauchenii, Rion!y. 

Tectibranches. G.APLYSIE.(APLYSIA) PL.XIV.

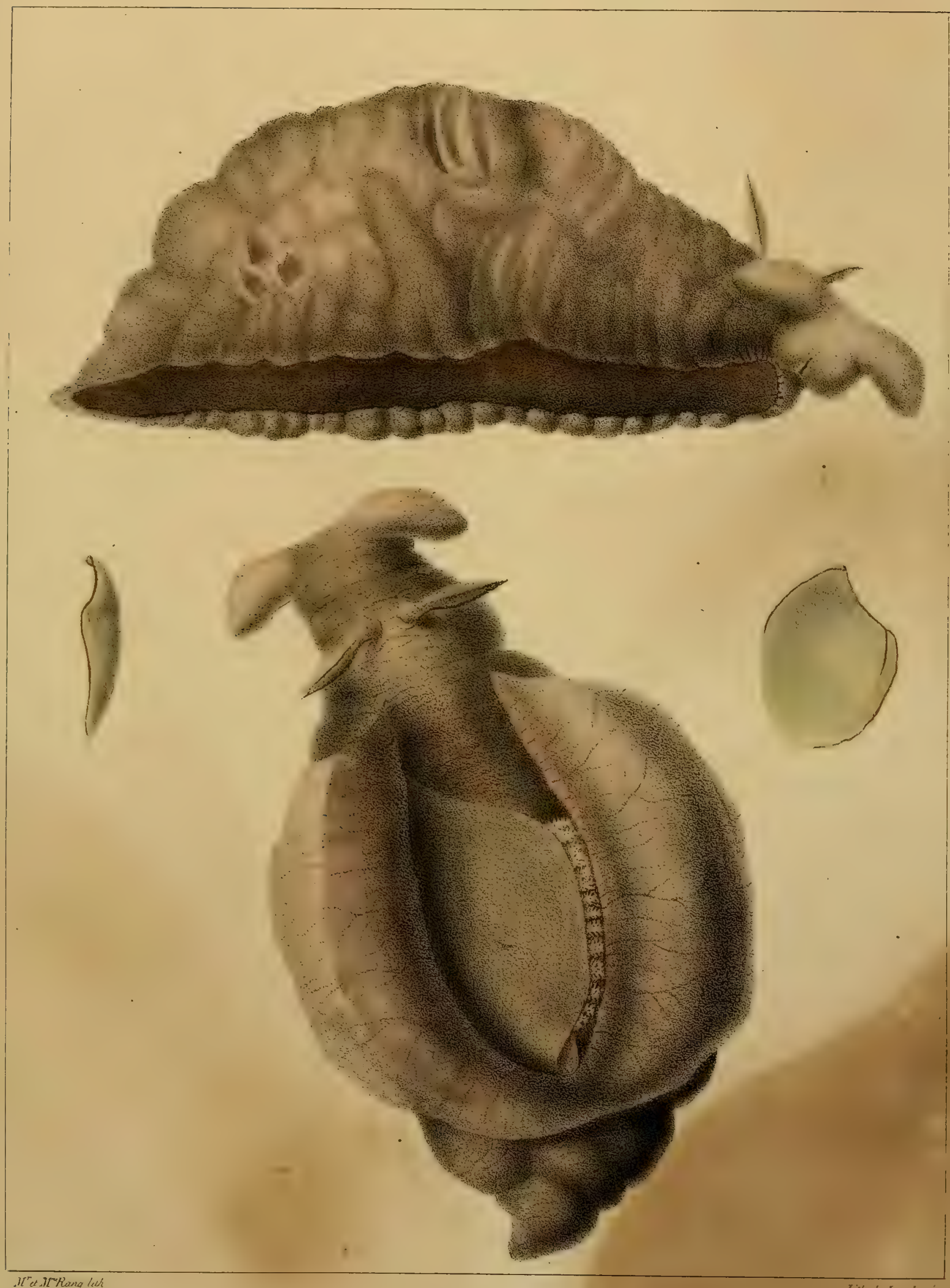


(1) 

Tectibranches.
G. APLYSIE . ( APLYSIA) PL XV

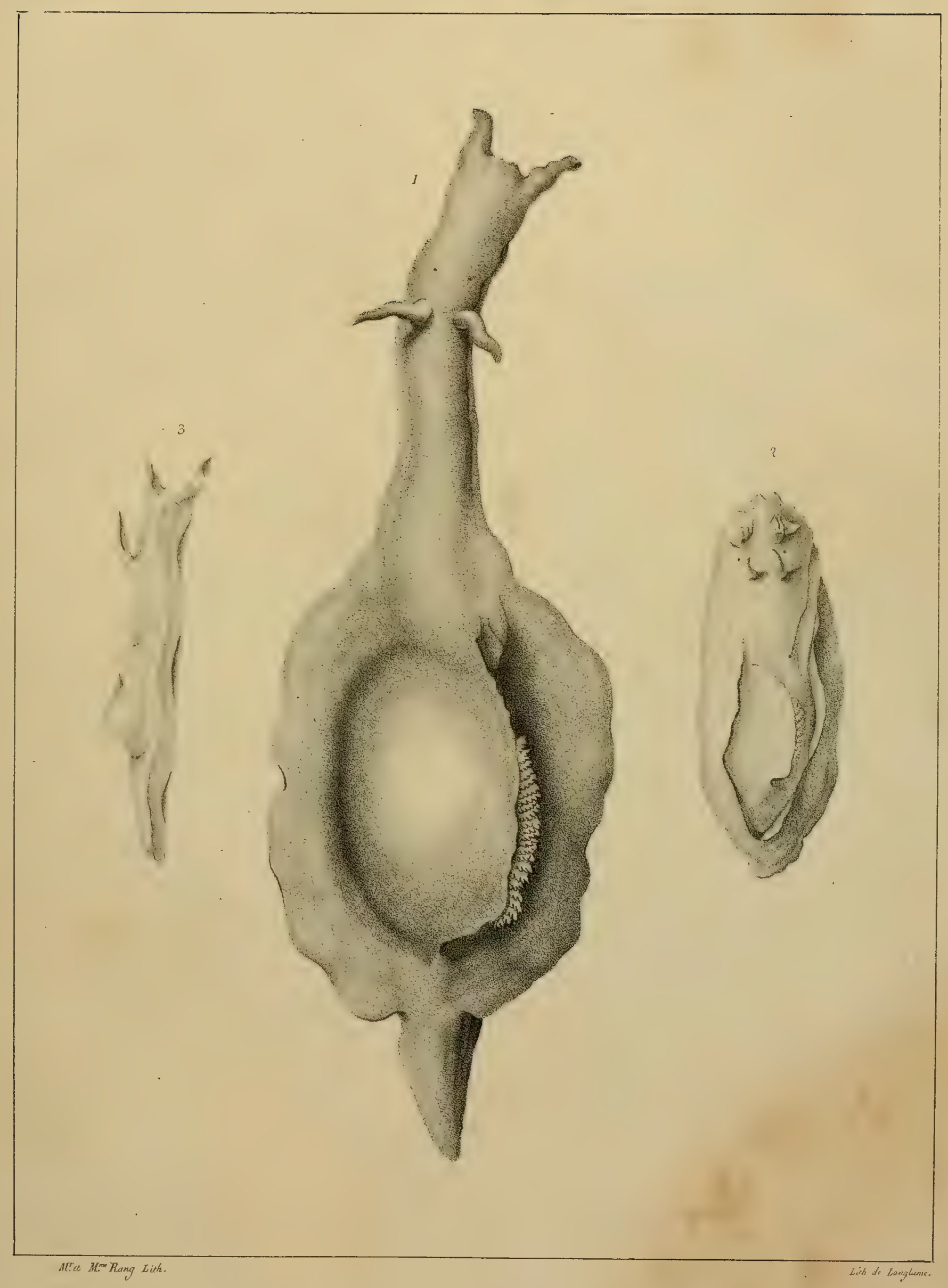

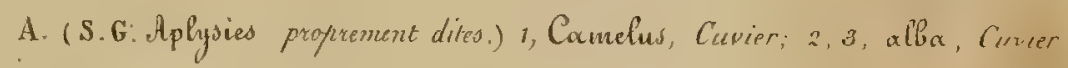





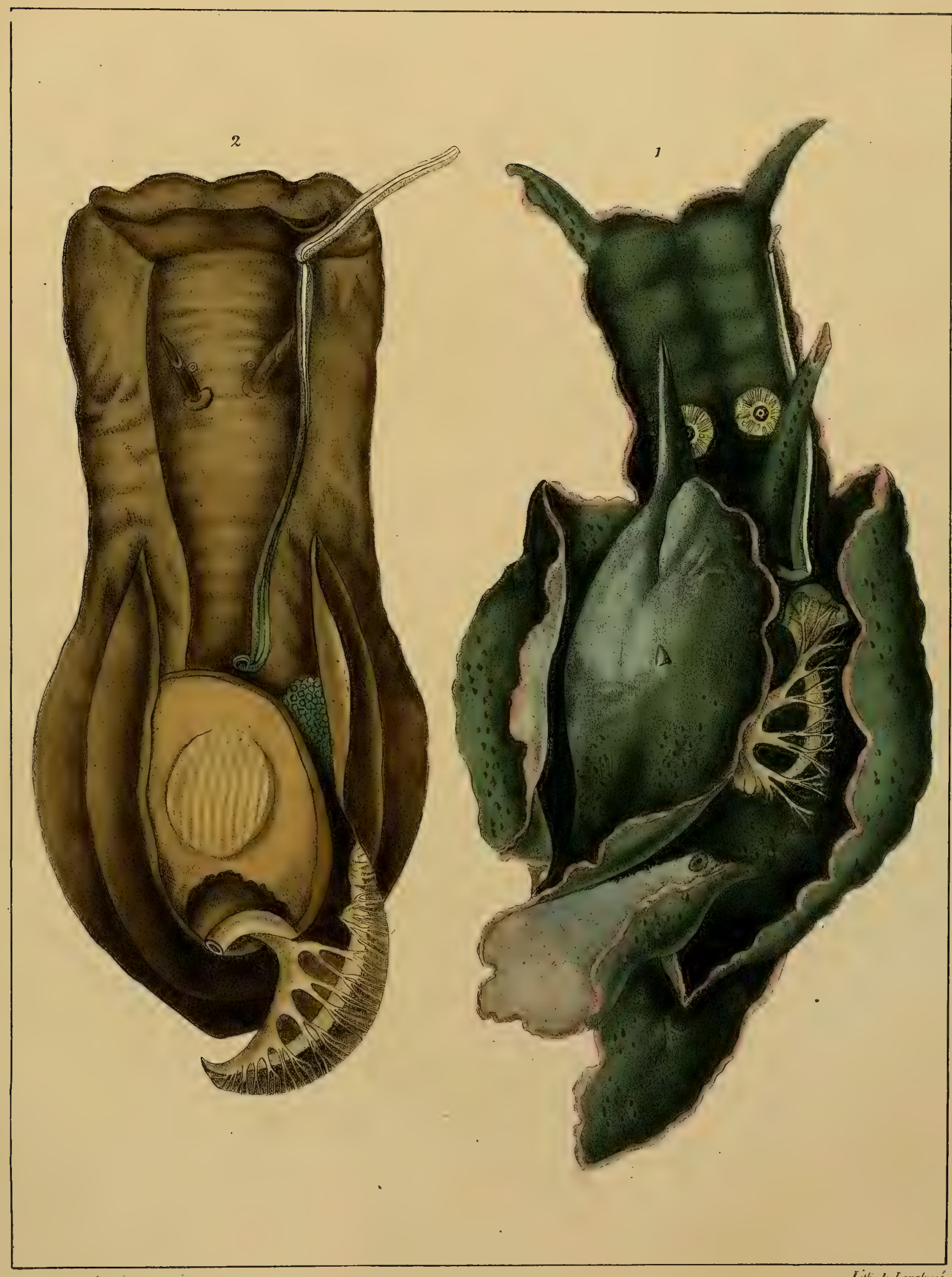

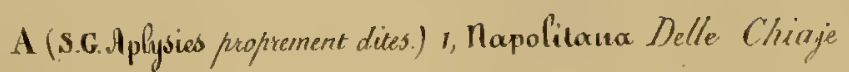
2, Polinim, Delle Chioge 
(2) 


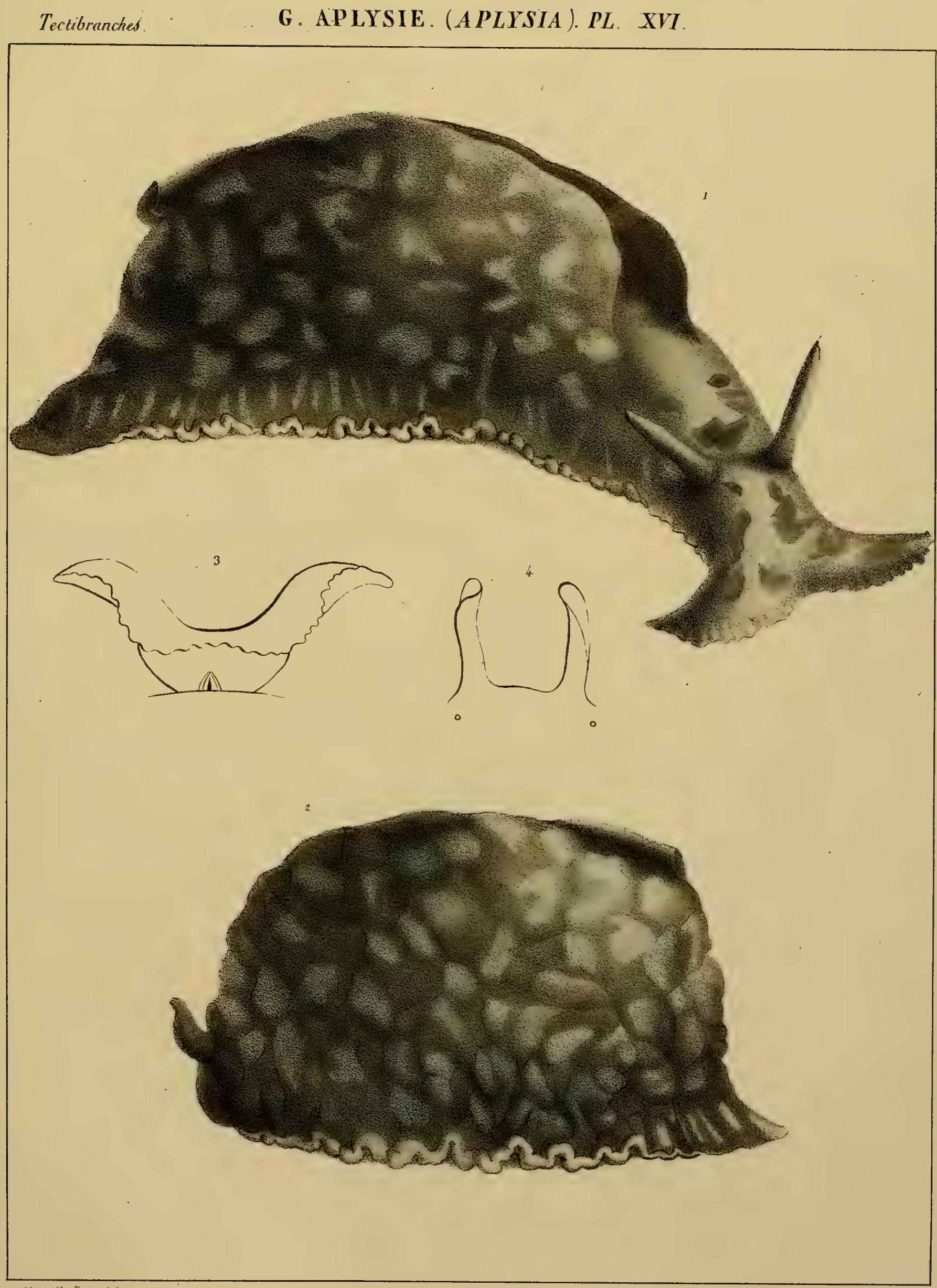

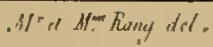

List. de Langlume

A (S.G: Mplyoics proprement dises.) depilous, Bohatsch. 



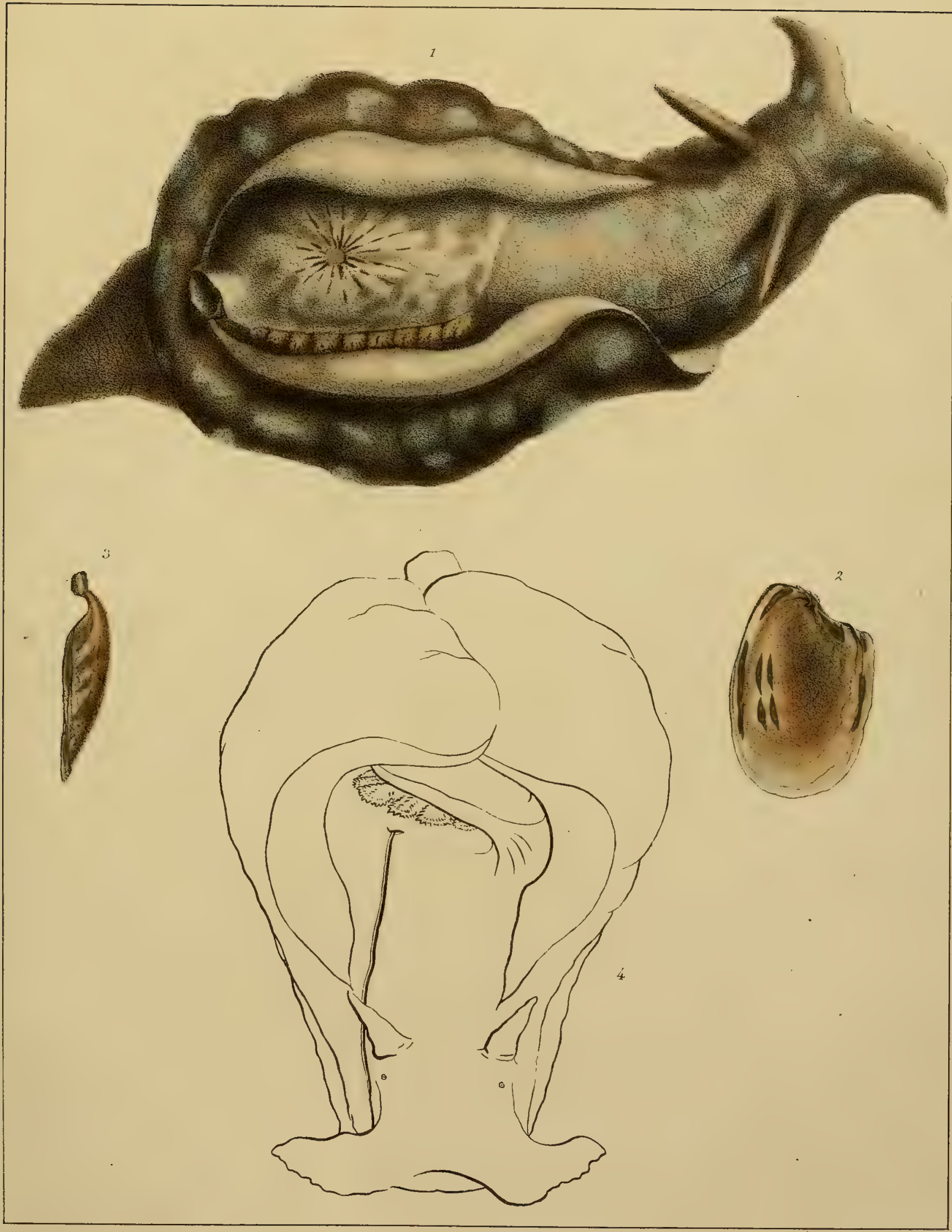

MI. He Mang del et lich Lith de Langlumé

A. ( S.G. Aplysies proprement ditess) Depilaus, Bohatuch. 



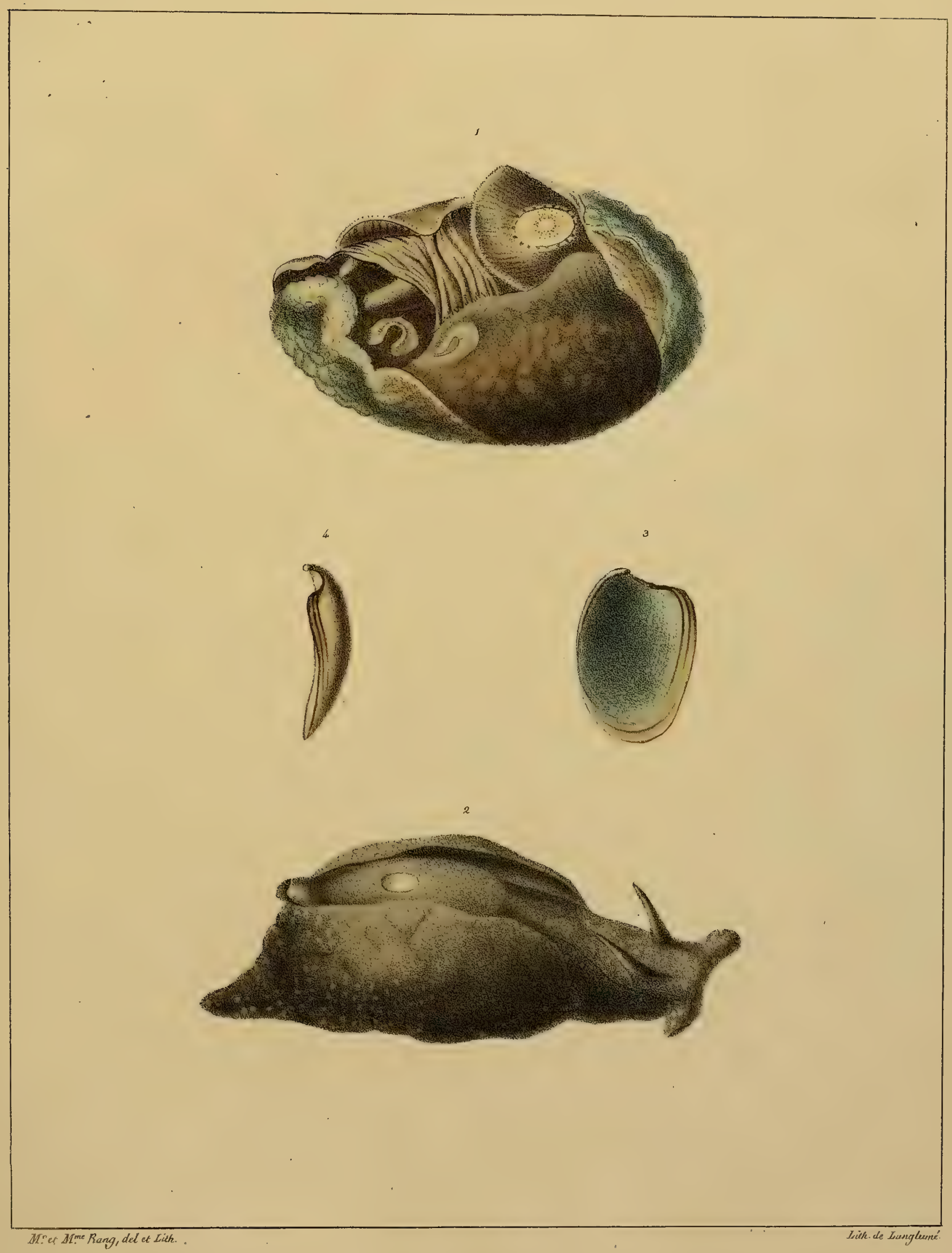

A. (S.G.Aplysies proprement dites.) , fusca, Tilesius; 2-4, punctata, Cuvier. 



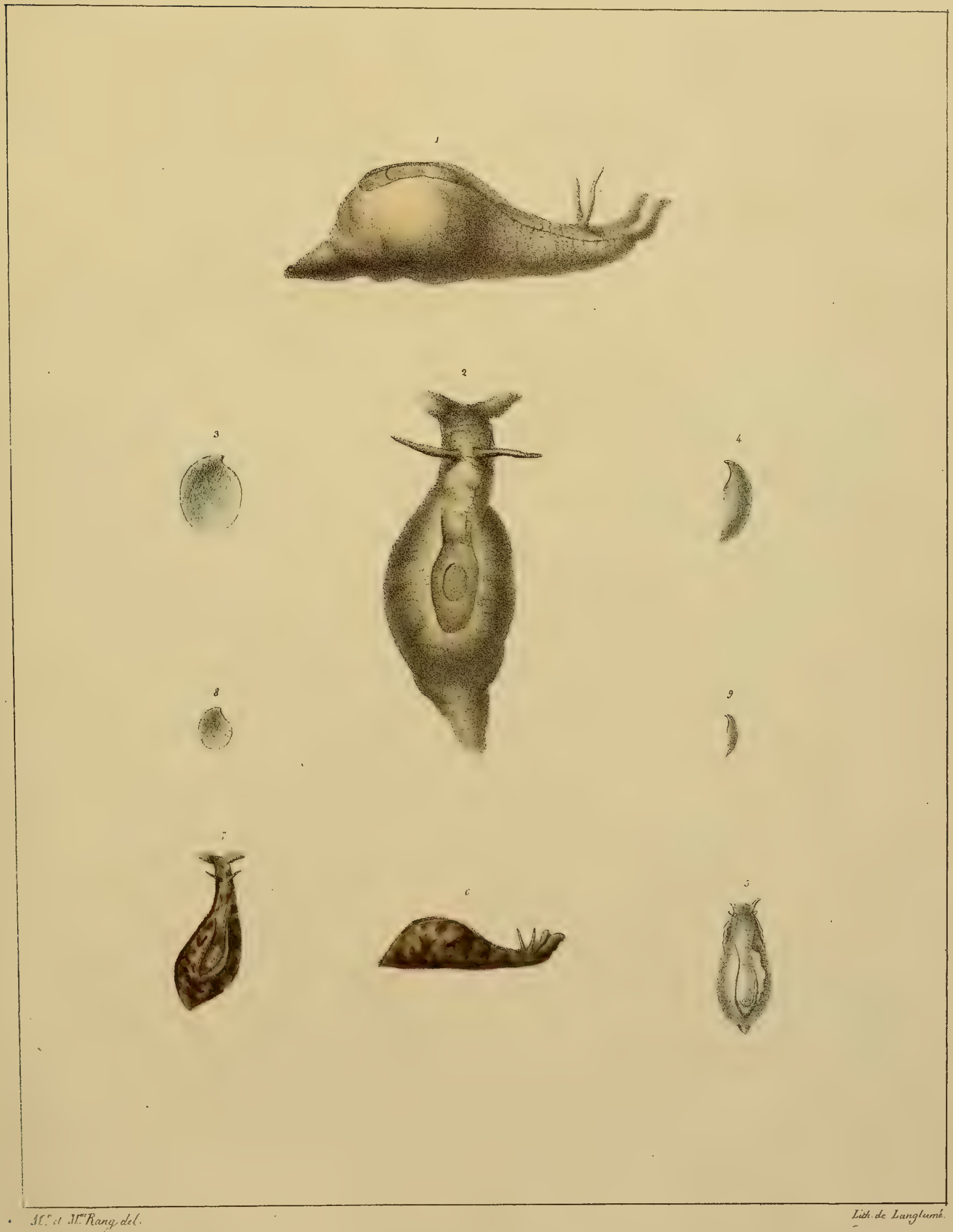

A. (s.c. Aplysies pruprement dites) 1-4, Pongicoruiw, Rang; S, sirescenb, Risso; 6-9, Ferussacii, Rang. 



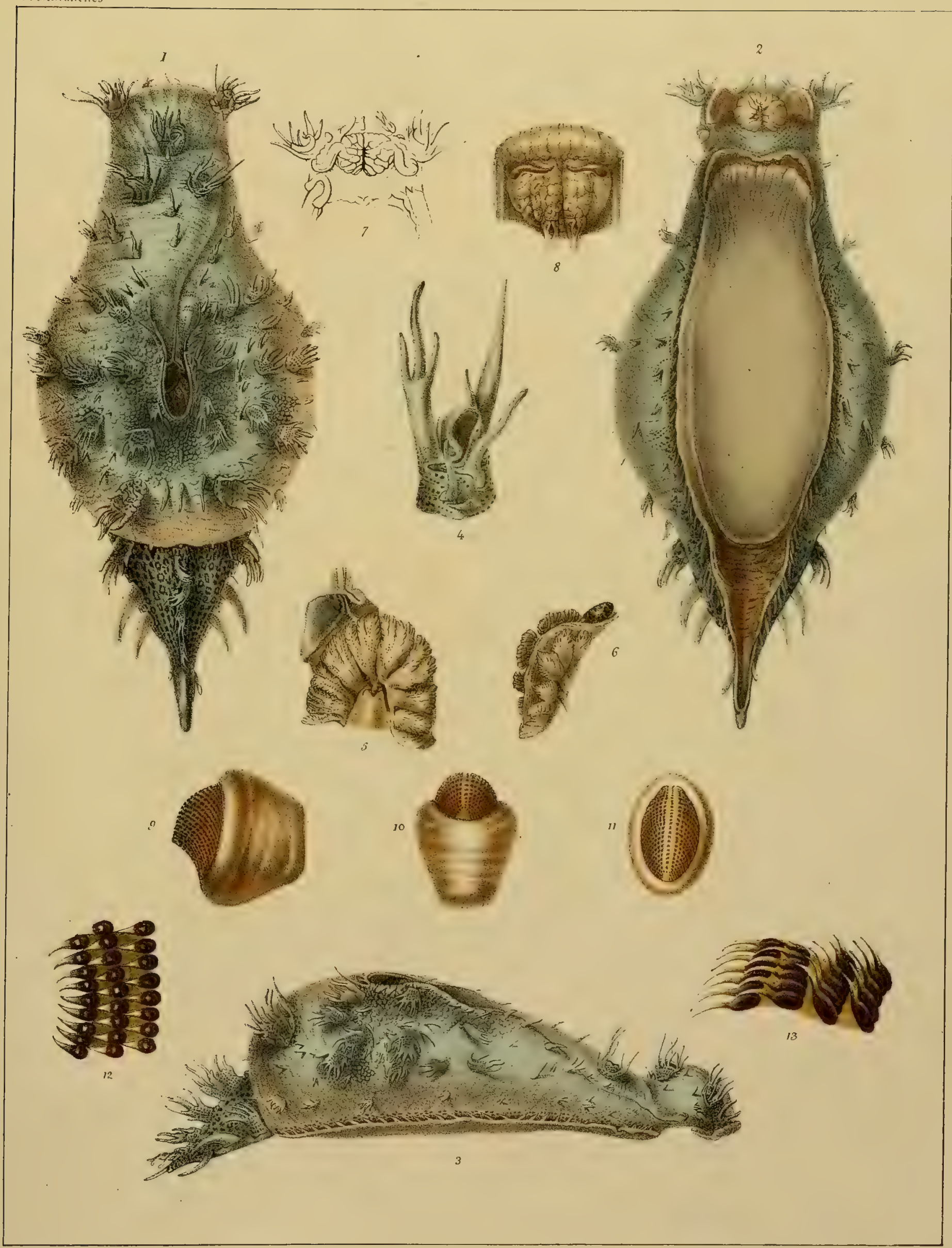

Aletien de Guinis 



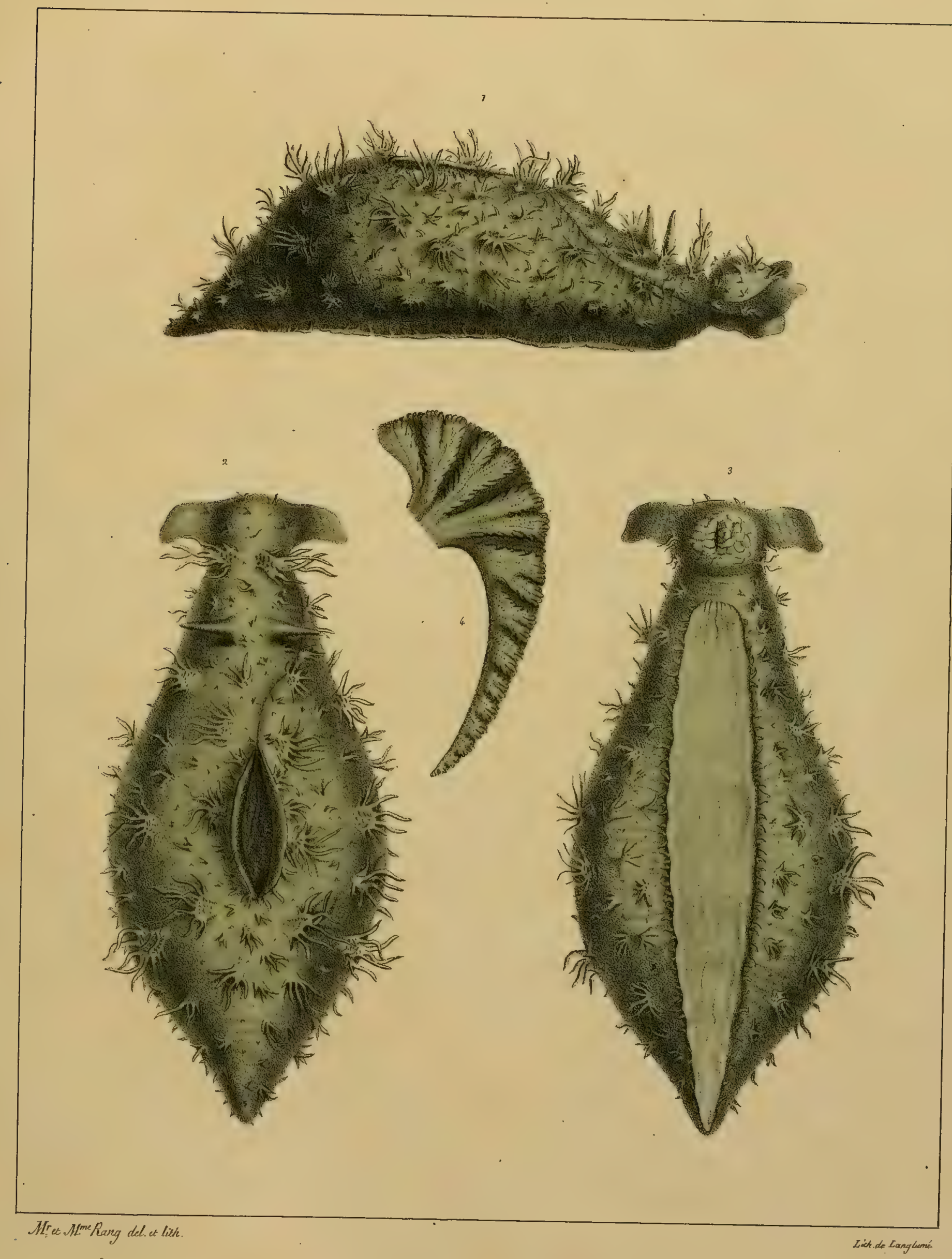

A. s.c. Aclésie.) Pleii, Rang 



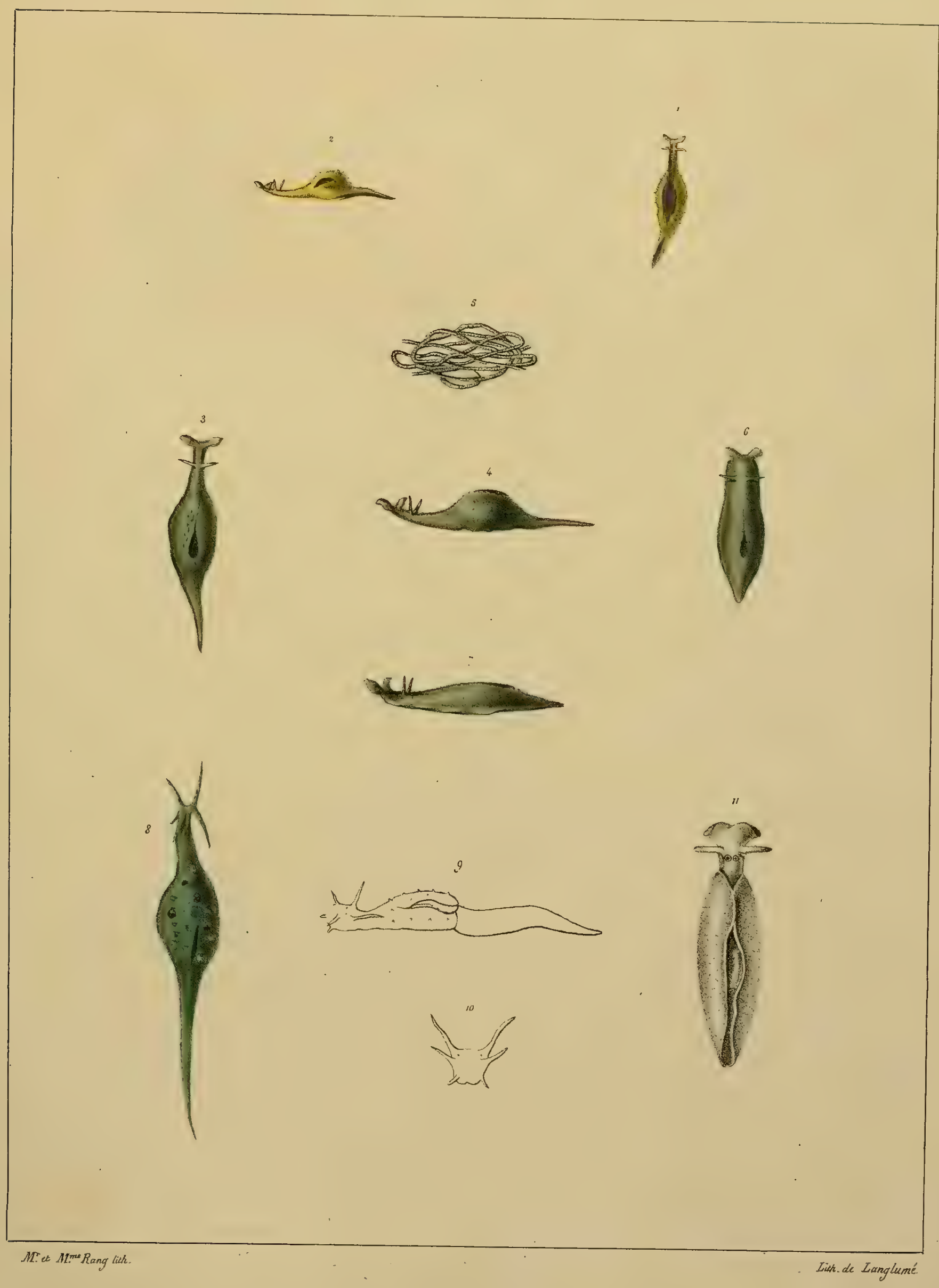

A. ( S.g. ACLÉSIE.)

1.2, Citrina, Rang; 3-5, undata, Rang"; 6,7, Rimacina, Bkainville; 8-10, longicauda, Quoy et Gaimard;"ll, virudis, Bousc. 

APIYSIE. (APLYSYA), hU HSATELLE.( BURSATELLA.) ACTEON. (ACTEON.) PL. XXII. Teclibranches.

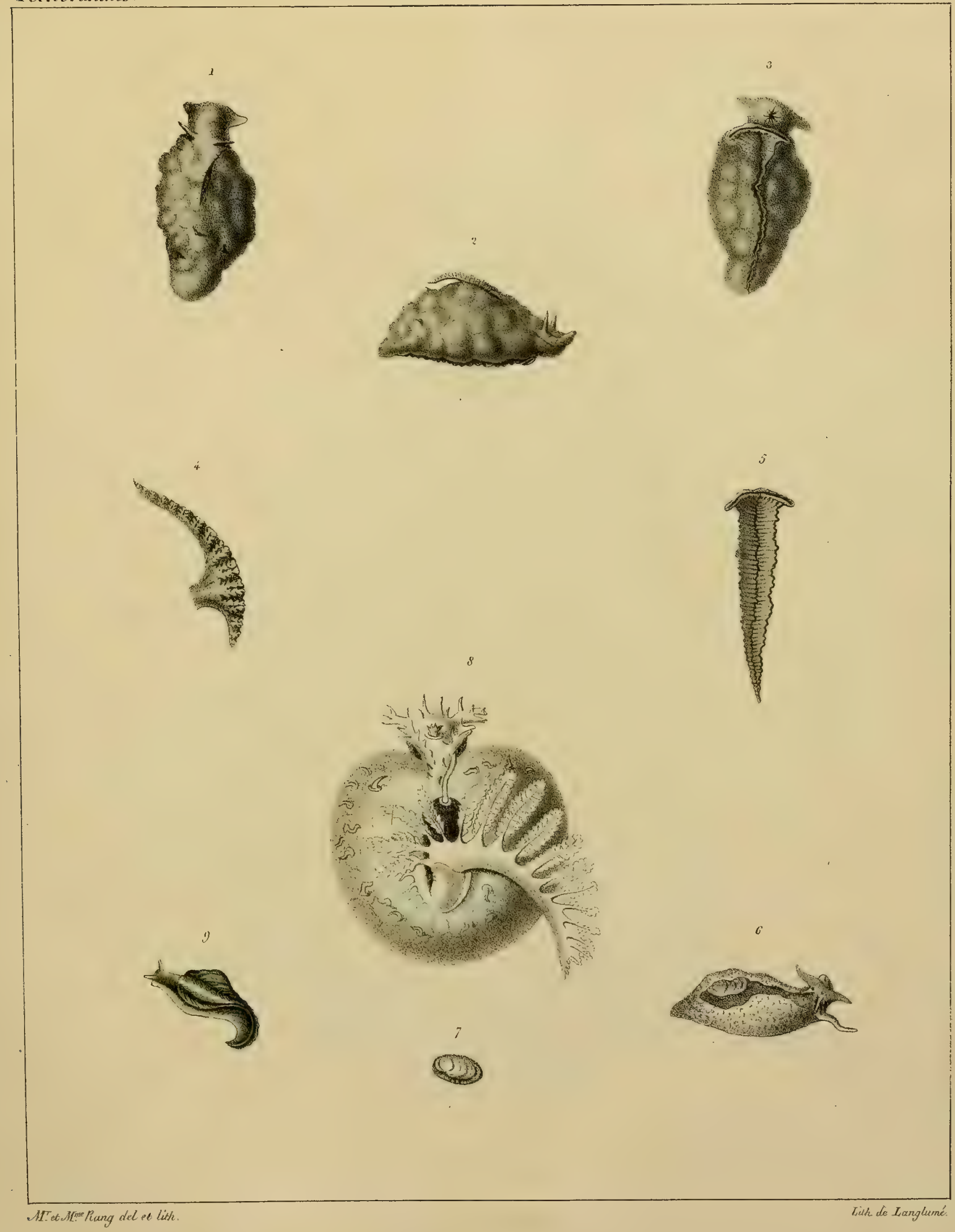

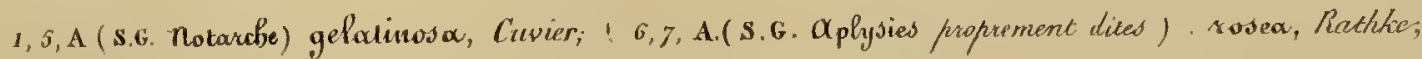
8, B., leachii, Blainville; g. ACT, viridis, Odken. 



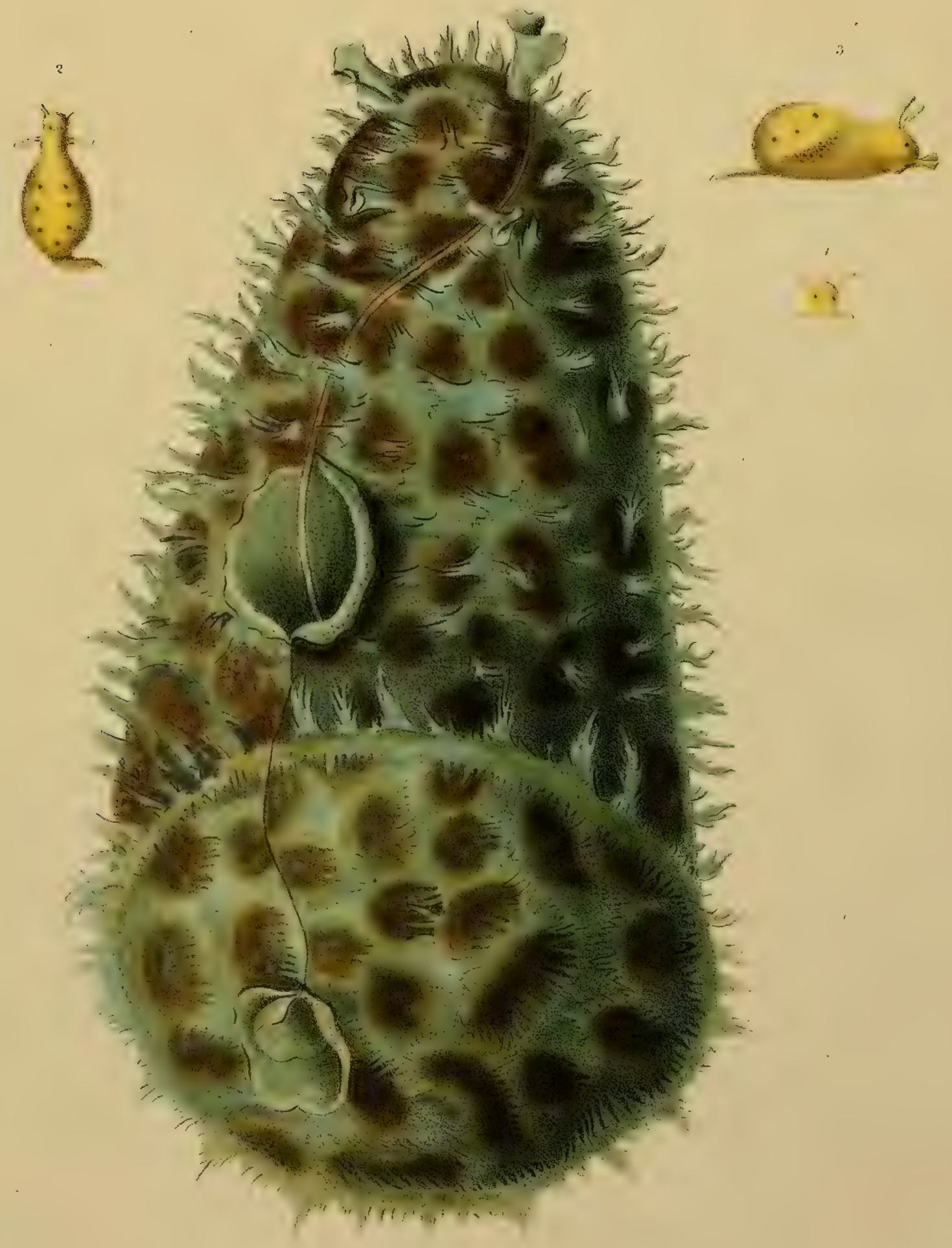

Ilme Rarmy, lith.

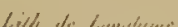

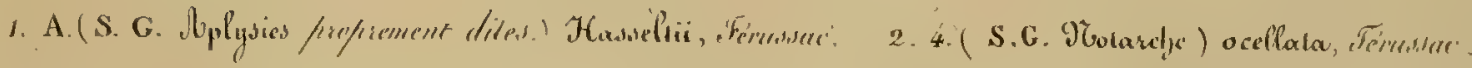






